\title{
VEGF and NOTCH in blood vessels, an intricate and fascinating interplay
}

Citation for published version (APA):

Caolo, V. (2011). VEGF and NOTCH in blood vessels, an intricate and fascinating interplay. [Doctoral Thesis, Maastricht University]. Datawyse / Universitaire Pers Maastricht. https://doi.org/10.26481/dis.20110511vc

Document status and date:

Published: 01/01/2011

DOI:

10.26481/dis.20110511vc

Document Version:

Publisher's PDF, also known as Version of record

\section{Please check the document version of this publication:}

- A submitted manuscript is the version of the article upon submission and before peer-review. There can be important differences between the submitted version and the official published version of record.

People interested in the research are advised to contact the author for the final version of the publication, or visit the DOI to the publisher's website.

- The final author version and the galley proof are versions of the publication after peer review.

- The final published version features the final layout of the paper including the volume, issue and page numbers.

Link to publication

\footnotetext{
General rights rights.

- You may freely distribute the URL identifying the publication in the public portal. please follow below link for the End User Agreement:

www.umlib.nl/taverne-license

Take down policy

If you believe that this document breaches copyright please contact us at:

repository@maastrichtuniversity.nl

providing details and we will investigate your claim.
}

Copyright and moral rights for the publications made accessible in the public portal are retained by the authors and/or other copyright owners and it is a condition of accessing publications that users recognise and abide by the legal requirements associated with these

- Users may download and print one copy of any publication from the public portal for the purpose of private study or research.

- You may not further distribute the material or use it for any profit-making activity or commercial gain

If the publication is distributed under the terms of Article $25 \mathrm{fa}$ of the Dutch Copyright Act, indicated by the "Taverne" license above, 


\title{
VEGF and NOTCH in
}

\author{
BLOOD VESSELS,
}

an INTRICATE and

FASCINATING INTERPLAY 
ISBN 9789461590497

Cover picture: "Blood Vessels from Inkjet Printers" by Abhinav Kaiser Printed by Datawyse BV I Universitaire Pers Maastricht

(C) Copyright: Vincenza Caolo, Maastricht 2011 


\title{
VEGF and NOTCH in
}

\author{
BLOOD VESSELS,
}

\author{
an INTRICATE and
}

\section{FASCINATING INTERPLAY}

\author{
DISSERTATION \\ to obtain the degree of Doctor at Maastricht University, \\ on the authority of the Rector Magnificus, Prof. dr. G.P.M.F. Mols, \\ in accordance with the decision of the Board of Deans, \\ to be defended in public on Wednesday $11^{\text {th }}$ May 2011 , at 16:00 hours \\ by \\ Vincenza Caolo
}

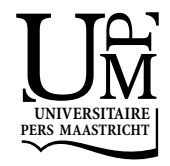




\section{Supervisor}

Prof. dr. M. J. Post

\section{Co-supervisor}

Dr. D.G.M. Molin

\section{Assessment Committee}

Prof. dr. J.G.R. de Mey (chairman)

Prof. dr. E.A.L. Biessen

Dr. G.J.J.M. van Eys

Prof. dr. M. Simons (Yale University School of Medicine, USA)

This research project has been supported by a Marie Curie FP6 Early Stage Research training grant MEST-CT-20005-020706. 


\section{Contents}

Chapter 1 General Introduction

Chapter 2 Feed-forward signaling by a membrane bound ligand receptor 25 circuit: The case of NOTCH-DELTA LIKE 4 in endothelial cells

Chapter 3 ADAM-10 is a novel mediator of VEGF-induced endothelial cell 45 function in angiogenesis and asso-ciated with atherosclerosis

Chapter 4 Developmetal coronary maturation is disturbed by aberrant cardiac VEGF-expression and NOTCH signaling

Chapter 5 Soluble JAGGED-1 inhibits Neointima Formation by attenuating NOTCH-HERP2 signaling

Chapter 6 General Discussion

Summary

Samenvatting

List of Abbreviations

151

Publications

154

Acknowledgments

155

Curriculum vitae

159 



\section{Chapter 1 \\ General Introduction}

NOTCH Signaling Pathway in Vascular Development

The NOTCH Signaling Pathway

NOTCH Signaling in ECs, a Direct Mediator of VEGF Signaling

VEGF-signaling

The role of VEGF and NOTCH in Artery/Venous Differentiation of Endothelial Cells VEGF and NOTCH in Endothelial Cell Sprouting

NOTCH in Vascular Smooth Muscle Cells (vSMC) Differentiation

Vascular Remodeling and NOTCH signaling

Chapter Outline 



\section{NOTCH Signaling Pathway in Vascular Development}

The formation of a functional vascular network is essential for the growth and maintenance of tissues. During embryogenesis the earliest stages of vascular development occur when endothelial cell (ECs) precursors (angioblasts) migrate and differentiate into blood islands, which then fuse to form the primitive capillary plexus in a process termed vasculogenesis ${ }^{1,2}$. The newly formed plexuses grow as a result of angiogenesis, i.e. vascular sprouting and tube formation by single ECs or by longitudinal division of existing capillaries, called intussusception. This process involves reorganization of the inter-endothelial cell junctions and central perforation of the bilayer, followed by interstitial pillar core formation and subsequent pericytes and myofibroblast invasion ${ }^{3,4}$. During the process of arteriogenesis, the formed channel of endothelial cells is covered by multiple layers of pericytes or smooth muscle cells (SMCs) to respectively generate small or large vessels of the vascular system. A large number of intercellular signaling pathways are implicated in these processes. These pathways include the families of vascular endothelial growth factors (VEGF), transforming growth factor- $\beta$ (TGF- $\beta$ ), fibroblast growth factors (FGF), platelet-derived growth factors (PDGF), angiopoietins and the ephrins. During the last decade several studies have made clear that the NOTCH pathway also plays an essential part in developmental neovascularization ${ }^{2}$.

The NOTCH pathway comprises a highly conserved intercellular signaling mechanism that is expressed in all metazoans and controls cell fate by cell-cell interaction $^{5}$. In vertebrates, components of the NOTCH signaling pathway are expressed in various organs including blood vessels. The importance of NOTCH signaling in vascular development has been underlined by the observation that mutations of $\mathrm{NOTCH}$ members can cause serious cardiovascular defects. Knockout mice of several components of the NOTCH pathway, such as Notch1, Notch1 plus Notch4 and Jagged1 all resulted in embryonic lethality related to vascular defects ${ }^{6,7}$. Mice homozygous for a single point mutation that prevents the intracellular processing of Notch1 resembles the null Notch1 phenotype, indicating that Notch1 signaling is essensial for embryonic viability and proper vascular morphogenesis ${ }^{8}$. Also both Delta-like 1 (DII1) deficient and Notch2-hypomorphic mice embryos presented with hemorrhage resulting from poor development of vascular vessels ${ }^{9}$, ${ }^{10}$. DIl4 mutant mice even exhibited embryonic lethal haploinsufficiency because of vascular remodeling defects ${ }^{11-13}$.

In human, the Alagille syndrome, an autosomal dominant disorder characterized by developmental abnormalities, is caused by mutations of the NOTCH ligand JAGGED ${ }^{14}$. The cardiac defects associated with Alagille syndrome include pulmonary outflow tract anomalies and tetralogy of Fallot. This congenital cardiac malformation predisposes to aortic valve calcification, which can impair blood flow 
through the valve. A second human disease, CADASIL (Cerebral autosomal dominant arteriopathy with subcortical infarcts ad leukoencephalopathy) is related to a mutation of the NOTCH-3 receptor ${ }^{15}$ and affects vascular smooth muscle cell (VSMC) development. CADASIL is the most common genetic disorder found in patients with stroke and vascular dementia ${ }^{16}$.

\section{The NOTCH Signaling Pathway}

The NOTCH pathway is an evolutionary highly conserved signaling system. Four different NOTCH receptors, NOTCH-1 to -4 , and five ligands, DELTA-LIKE (DLL) -1, -3, -4 and JAGGED (JAG) $-1,-2$, have been identified in vertebrates. The NOTCH members are single-pass transmembrane proteins. NOTCH receptors are synthesized as single-chain precursors that, after glycosylation by protein o-fucosyl transferase (POFUT1) in the endoplasmic reticulum, are processed into noncovalently linked extracellular (NECD) and intracellular (NICD) domains in the transGolgi $^{17-19}$. The NECD is characterized by 29-36 epidermal growth factor (EGF)-like repeats which mediate the interaction with the ligand, and three cysteine-rich NOTCH/LIN12 repeats that prevent ligand independent signaling ${ }^{17-19}$. The NICD is characterized by a RAM (RBP-Jk associated module), a motif containing a NLS (nuclear localization sequence) that facilitates translocation and constitutes the primary binding domain to RBP-Jk. The RAM motif is followed by six tandem ankyrin repeats (ANK), which are highly conserved among NOTCH proteins of various species and mediate protein-protein interaction. Downstream of the ANK repeats lies a glutamine-rich domain (OPA), followed by a C-terminal PEST (proline/glutamic acid/serine/threonine-rich) sequence which facilitates rapid turnover of the protein

${ }^{20}$. The ligands, also referred to as DSL (ㅌelta/Serrate/Lag-2) are characterized by an extracellular motif, which mediates the binding with the receptor, EGF-like repeats, and a cytoplasmatic domain containing a conserved C-terminal PDZ (postsynaptic density; discs large; zonula occludens-1) -binding motif ${ }^{18}$. The receptor-ligand interaction induces two proteolytic cleavages of the receptor. The first is mediated by extracellular proteases, known as A Disintegrin And Metalloprotease (ADAM), TNF- $\alpha$ Converting Enzym (TACE) or kuzbanian. Subsequently, the $\gamma$-secretase complex mediates a second proteolytic cleavage that releases the NOTCH Intracellular Domain (NICD) from the membrane. Next, the NICD translocates to the nucleus, where it associates with the DNA binding protein RBP-Jk (also named CSL after mammal CBF1, Drosophila $\mathrm{Su}(\mathrm{H})$, and Caernorhabditis elegans LAG-1) and its co-activator Mastermind (MAML1) to initiate the transcription of its downstream targets, such as the basic Helix-Loop-Helix proteins Hairy/Enhancer of Split (HES) and Hairy Related Transcription factors (HRT, HERP, HEY) ${ }^{18,21-24}$, which in turn regulate the transcription of downstream genes. 


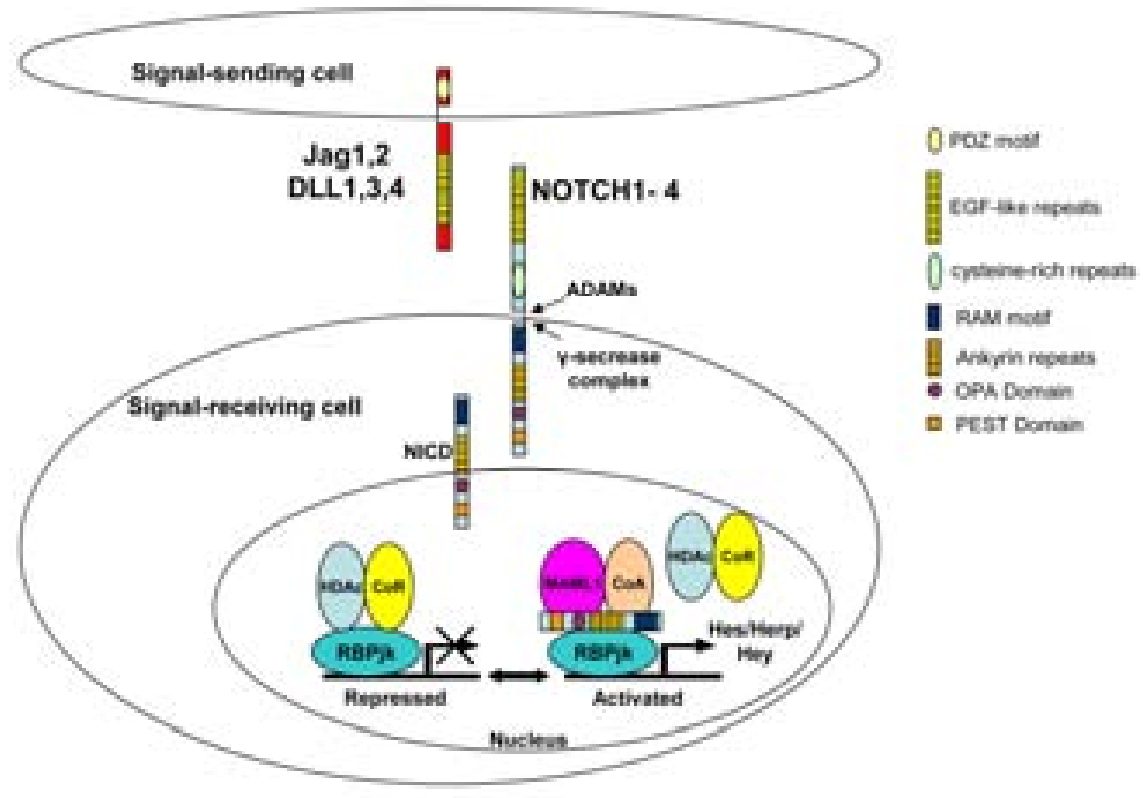

Figure 1 Schematic Representation of NOTCH signaling pathway

Mammals possess 4 NOTCH receptors (NOTCH1-4) and five ligands (Jagged1 and 2, and Delta-like1, 3, 4). NOTCH signaling is triggered upon receptor-ligand interaction, which induces two sequential proteolytic cleavages. The first cleavage, in the extracellular domain, is mediated by metalloproteinases of the ADAM family, and the second, within the membrane domain, is mediated by the $\gamma$-secretase complex. The second cleavage allows the release and translocation of the NOTCH intracellular domain (NICD) to the nucleus where it associates with the RBPjk co-transcription factor. The binding of NICD to RBP-Jk leads to transcriptional activation of the HES and HERP/ HEY NOTCH target genes by displacement of a co-Repressor ( $\mathrm{COR}$ ) and recruitment of the co-Activator, Mastermind Like protein (MAML1). NOTCH ligand and receptor domain compositions are also illustrated in the figure.

\section{NOTCH Signaling in ECs, a Direct Mediator of VEGF Signaling}

Activation of NOTCH signaling in the endothelium controls important processes such as arterial/venous differentiation and sprouting of vessels. Within this context the role of NOTCH signaling is intertwined with another essential regulator of vascular development; Vascular Endothelial Growth Factor (VEGF) ${ }^{25,26}$.

\section{VEGF-signaling}

The VEGF family members are secreted as dimeric glycoproteins of approximately $40 \mathrm{kDa}$. There are five characterized VEGF subgroups in mammals (VEGF-A through 
VEGF-D, and placenta growth factor, (PIGF)). Homologues of VEGF have also been identified in the genome of the parapoxvirus Orf, VEGF-E, and in snake venom, VEGF-F. Alternative mRNA splicing of VEGF-A in human generates six different VEGF-A isoforms: VEGF- $A_{121}$, VEGF- $A_{165}$ and VEGF- $A_{189}$ being the most important, which are represented by VEGF- $A_{120}$, VEGF- $A_{164}$ and VEGF-A $A_{188}$, respectively, in the mouse. The VEGF ligands display different interactions with the three VEGF receptor tyrosine kinases (RTK): (VEGFR-1/FIt1, VEGFR-2/KDR/Flk1 and VEGFR-3/FIt4) ${ }^{27-29}$. The activities of VEGF-A isoforms are dictated by their different ability to interact with the VEGF co-receptors, such as NPs (neuropilins) and HSPGs (heparin sulphate proteoglycans) $)^{30}$. Heparan-sulfate proteoglycans and the neuropilins bind VEGFs but do not induce biological responses on their own in the absence of the tyrosinekinase receptors ${ }^{27}$. VEGF-A $A_{121}$ lacks the heparin binding domain and is freely diffusible; whereas VEGF- $A_{189}$ is strongly bound to the extracellular matrix; VEGF$A_{165}$ has intermediate properties. VEGF- $A_{165}$ is secreted, however a significant fraction remains bound to heparan sulphates of matrix proteins situated on the cell surface. The VEGF importance in regulating vascular development has been well established $^{31-33}$. VEGF-A haploinsufficiency is lethal in mouse embryos, presenting with a prominent vascular phenotype including a reduced number of blood vessels ${ }^{32,33}$. On the other hand, increased VEGF-A levels also lead to severe abnormalities in the heart and subsequently embryonic lethality in mice ${ }^{34}$.

Among the VEGF receptors, VEGFR-1 and VEGFR-2 are expressed in vascular endothelium of both arterial and venous origin, whereas VEGFR-3 expression is restricted to lymphatic endothelium. VEGFR-1 is also abundant in macrophages/monocytes. VEGFR-1 and -2 are structurally similar, consisting of an extracellular domain with seven immunoglobulin Ig-like motifs, a single transmembrane domain, a juxtamembrane domain, a kinase domain split by a kinase insert, and a carboxyl terminus. The VEGF ligands display different interactions with the VEGF Receptors (Figure 2). VEGF-A and VEGF-F (a snake isoform) interact with both VEGFR-1 and VEGF-R2; VEGF-B and PIGF interact exclusively with VEGFR-1, VEGF-E (a viral isoform) binds only to VEGFR-2, whereas VEGF-C and VEGF-D interact with VEGFR-2 and R-3 $3^{28,35}$.

It is generally accepted that VEGFR-2 is the major mediator of the VEGF induced effects in endothelial cells, showing a stronger activation than VEGFR-1, and stimulating a variety of signaling pathways and biological responses in endothelial cells $^{36}$. VEGFR-2 deficient mice fail to form blood-island and show impaired vasculogenesis $^{37}$. VEGFR-1 is considered to be a kinase-impaired RTK, since autophosphorylation of its tyrosines is rather poorly induced. This poor kinase activity of VEGFR-1 is probably the result of an aspartic acid to aspargine mutation in the activation loop of the tyrosine kinase domain ${ }^{38}$. The redundancy of the VEGFR-1 is illustrated by minor effect of the deletion of the tyrosine kinase domain on vascular development in mice ${ }^{39}$. The observation that targeted mutation of PIGF, 
as a specific VEGFR-1 ligand, does not affect embryonic vascular development further supports the redundancy of VEGFR-1 signaling in this context ${ }^{31}$. Moreover, VEGFR-1 deficient embryos show normal endothelial differentiation but an increased number of endothelial cells and disorganized primary blood vessels ${ }^{39,40}$, likely resulting from excessive VEGF/VEGFR-2 signaling. These studies indicate VEGFR-1 as a negative regulator of endothelial proliferation. These observations together with the biochemical characteristic of its tyrosine kinase domain suggest that during embryogenesis, VEGFR-1 in ECs may serve as a decoy receptor, effectively reducing the availability of VEGF for the VEGFR-2 receptor, rather than as a signaling receptor.

It is worth to note that despite its secondary role in ECs, active VEGFR-1 is the sole VEGF receptor expressed on the surface of circulating monocytes ${ }^{41}$, where it mediates chemotaxis in response to VEGF or PIGF-1 ${ }^{42}$.

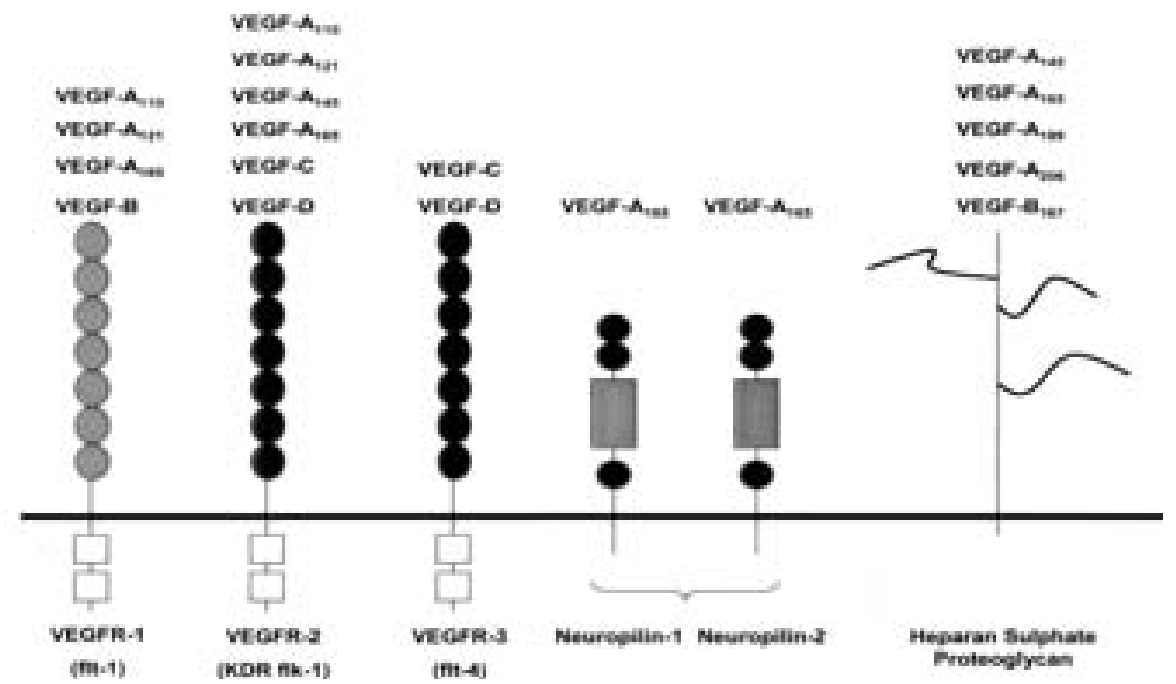

\section{Figure 2 Growth Factors and Receptors of the VEGF family}

The three signaling tyrosine-kinase receptors of the VEGF family (VEGFR-1, VEGFR-2, and VEGFR-3), the accessory isoform specific receptors neuropilin-1 and neuropilin-2, and VEGF binding heparansulfate proteoglycans are displayed together with their major structural features. The different isoforms of the VEGF family members that bind to each of these cell membrane proteins are also shown (modified after Hoeben Pharmacological Reviews 2004).

\section{The role of VEGF and NOTCH in artery/venous differentiation of endothelial cell}

Although blood flow has for long been considered the major factor to regulate the differentiation of arteries and veins, recent studies have shown that genetic prepatterning, mainly mediated by the NOTCH pathway, play a primary role in inducing arterial-venous differentiation. 
The importance of the NOTCH signaling pathway in regulating arterial differentiation was initially shown in Zebrafish ${ }^{43,44}$. NOTCH signaling deficient embryos showed a poorly formed dorsal aorta and posterior cardinal vein. These embryos also showed loss of arterial marker expression, such as EphrinB2, and ectopic expression of venous markers in the arterial compartment. Reduction of VEGF-A signaling also resulted in loss of arterial marker in the dorsal aorta and ectopic expression of venous markers, whereas injection of VEGF-A mRNA induced the expression of the arterial marker EphrinB2 in the posterior cardinal vein ${ }^{43}$. Moreover, induced expression of NOTCH-1 in VEGF-A-deficient embryos rescued the expression of EphrinB2, whereas injection of VEGF-A mRNA into NOTCH signaling deficient embryos was unable to restore arterial differentiation ${ }^{43}$. These studies provide evidence that both $\mathrm{NOTCH}$ and VEGF are involved in a signaling cascade that is essential for arterial-venous differentiation in which NOTCH signaling acts downstream of the VEGF pathway.

The VEGF-A/NOTCH axis in regulating arterial endothelial differentiation has also been confirmed in mice. Vegf164 overexpression in cardiomyocytes and primary embryonic endothelial cells ${ }^{45}$ leads to arterial differentiation with an increased EphrinB2/EphB4 ratio. Likewise in murine vasa nervorum, Schwann cell derived Vegfa also stimulates arterial markers ${ }^{46,47}$. NOTCH activation induced arterial differentiation in mice as shown by loss and gain of function studies. Surviving D/l4 heterozygous mice displayed a lack of arterial markers in their vasculature ${ }^{11-13,48}$, as well as NOTCH signaling deficient mice such as the RBP-Jk and Hey1 and Hey2 double-mutants ${ }^{11-13,49,50}$. Studies in cultured endothelial cells confirmed that DLL4induced NOTCH signaling up-regulates EphrinB2 expression ${ }^{51}$. Furthermore, the overexpression of activated NOTCH-4 (NICD-4) in adult mice also induced the expression of EphrinB2 in the venous compartment resulting in arteriovenous malformation and this phenotype was reversible after repression of transgene expression $^{52}$.

The close interaction of VEGF and NOTCH signaling in arterial differentiation has been defined in mammal cells. Liu et al. ${ }^{53}$ showed that NOTCH-1 receptor and DLL4 ligand are induced by VEGFA in human arterial but not in venous endothelial cells. VEGF-A induced DLL4, NOTCH-4, EphrinB2 and downregulation of venous markers COUP-TFII and EphB4 expression was also described for mouse embryonic ${ }^{54}$ and human bone-marrow derived mesenchymal stem cells ${ }^{55}$. Also ablation of the venous marker and repressor of NOTCH signaling, COUP-TFII, in endothelial cells enabled veins to express $\mathrm{NOTCH}$ signaling components, acquiring an arterial phenotype, whereas COUP-TFII ectopic expression resulted in fusion of arteries and veins (AVshunts) in transgenic mouse embryos ${ }^{56}$.

As anticipated above, besides genetic factors, enviromental factors such as hemodynamics and oxygen tension also intervene in defining artery and venous identity. Blood vessels preserve a certain degree of plasticity which allows them to 
respond to external stimuli as fluid mechanic forces and lack of oxygen. Masumura et al. ${ }^{57}$ have reported that shear stress regulates the arterial-venous specification of murine embryonic stem (ES) cells-derived ECs. They showed that EphrinB2 expression increased when these cells were exposed to shear stress, whereas EphB4 expression decreased. However, the prevention of the effects in the presence of VEGFR-2 and NOTCH signaling inhibitors showed that shear stress induced arterialdifferentiation requires the VEGF-NOTCH signaling pathway. Similarly, low oxygen levels can trigger VEGF expression through the hypoxia inducible factor (HIF) signaling cascade ${ }^{58-61}$. Moreover, promoter analysis revealed that Hey1, Hey2 and DII4 are induced by HIF-1 alpha and NOTCH signaling ${ }^{62}$ and hypoxia-mediated DII4 and Hey2 upregulation repressed COUP-TFII expression in endothelial progenitor cells (EPCS).

\section{VEGF and NOTCH in Endothelial Cell Sprouting}

During angiogenesis, new capillaries sprout from pre-existing blood vessels. In this process, specialized endothelial cells, called tip cells, react to a VEGF gradient ${ }^{63,64}$ by migrating and extending filopodia. This response is highly localized as the stalk cells -that follow the tip cell- already respond differently to VEGF $^{26}$. Several recent studies have shown that NOTCH signaling plays an important role in regulating the formation and function of these tip cells. Endothelial tip cells present with high levels of VEGFR-2 ${ }^{65}$. In response to VEGF stimulation DII4 expression is upregulated in these cells ${ }^{66,67}$. The tip cell expressing DII4 interact with the neighbouring cells, thereby activating NOTCH signaling, which in turn prevents migration and filopodia extension that will functionally define these neighbouring cells as stalk cells. Thus, in the absence of NOTCH signaling, endothelial cells continue to form sprouts in response to VEGF resulting in more sprouts and subsequently, branches per blood vessel. This indeed has been observed in different model systems such as mouse retina and hindbrain ${ }^{66,68-70}$, zebrafish embryos ${ }^{71,72}$, and xenograft tumor models ${ }^{70,}$ 73,74 . Consistently, D/l4+/- mice ${ }^{66,68,69}$ present serious vascular defects in the retinal vasculature with excessive capillary density, diameter and filopodia extension of endothelial cells, while similar defects have been observed with NOTCH inhibition either in an inducible NOTCH-1 knockout ${ }^{68}$, pharmacological blockade by anti-DII4 antibodies ${ }^{69,70}$ or $y$-secretase inhibitors ${ }^{66,68}$. Stalk cells that are unchecked by DLL4 also remain highly proliferative as shown in DII4 or RBP-Jk morphant zebrafish embryos $^{72}$.

\section{NOTCH in Vascular Smooth Muscle Cells Differentiation}

Besides affecting arterial ECs differentiation, NOTCH signaling has been reported to specify arterial-venous identity of vascular SMCs as well ${ }^{75}$. Although several reports 
have been published during the last years with respect to NOTCH and SMC differentiation, the results have been contradictory.

Among the receptors, Notch3 is expressed in vascular SMCs from arteries but not in those from veins ${ }^{75}$. Notch3-/- mice show serious arterial defects, such as enlarged arteries with a thinner layer of SMCs ${ }^{75}$ that are poorly differentiated ${ }^{75-77}$ compared to wild-type animals. This is consistent with in vitro data showing that ligand induced NOTCH signaling up-regulated the SMA (Smooth Muscle Actin) and SMMHC (Smooth Muscle myosin heavy chain) expression, promoting VSMC differentiation ${ }^{78-80}$ via RBP-Jk. Among the ligands, endothelial Jag1 appears to be essential in regulating vascular morphogenesis by inducing NOTCH signaling in the neighbour SMC. Endothelial-specific deletion of Jag1 caused serious cardiovascular abnormalities and embryonic lethality in mice ${ }^{81}$. Surprisingly, these embryos differ from embryos in which general NOTCH signaling has been abrogated as they still present with normal NOTCH activation and arterial differentiation of the endothelium. This suggest that endothelial Jag1 mainly acts by inducing the differentiation of the adjacent SMCs ${ }^{81}$. NOTCH3 activation by endothelial Jag1 is further supported by enhanced expression of NOTCH3 in SMC co-cultured with Jag1 expressing endothelial cells, suggesting a positive feedback loop. Indeed, Liu et al ${ }^{79}$, demonstrated that activated NOTCH-3 signaling initiates a positive feedback loop that promotes its own expression and propagated NOTCH signaling to other SMCs, meanwhile inducing SMC differentiation markers, such as $\alpha$-SMA, CALPONIN and $\mathrm{SM}-\mathrm{MHC}$. These data are in contrast with other studies in which NOTCH target genes have been reported to antagonize SRF (Serum Response Factor) and myocardin, the main transcription regulators of VSMC gene expression and SMC differentiation ${ }^{82}$. The controversy between these reports could relate to the balance between NOTCH ICD that induces the expression of VSMC markers and, the contemporary expression of NOTCH target genes such as Hey1 and Hey2 that would turn off these factors as part of a negative feedback loop. The validity of this model

has been experimentally addressed by using ECs and SMC cocultures ${ }^{83,84}$. Hey1 and Hey2 were indeed able to repress SMA expression and to antagonize the NOTCHinduced SMA expression in vitro by decreasing NOTCH ICD/RBP-Jk binding and transactivation of the SMA promoter.

\section{Vascular Remodeling and Notch Signaling}

In the adult vascular remodeling can occur upon vascular damage (inward remodeling) or altered blood flow (outward remodeling). During inward remodeling excessive migration and proliferation of SMCs in response to vascular injury induces the formation of a thickened layer of arterial intima from the media referred to as neointima. Increased blood flow is the main trigger of an endothelial-monocyte driven expansion of the vessel wall referred to as outward remodeling. This process 
in which the increase in both ECS and SMCs number facilitate the expansion of the vessel is also defined as arteriogenesis.

As regulator of SMC proliferation and migration, the NOTCH signaling pathway has been associated with both arteriogenesis and neointima formation. With respect to collateral growth, Limbourg et al. $^{85}$ have underlined the importance of NOTCH signaling in postnatal arteriogenesis in response to hindlimb ischemia. Mice heterozygous for Dll1 showed absence of endothelial NOTCH activation and EphrinB2 induction after hindlimb ischemia, with subsequent lack of arterial collateral growth and impairment of blood flow recovery of the hindlimb. Similarly, postnatal angiogenesis and blood flow recovery was impaired in haplo-insufficient general or endothelial specific Notch1+/- mice ${ }^{86}$. In the arterial response to injury, the effects of NOTCH signaling and of its downstream targets on VSMC differentiation, migration and proliferation make it a suitable candidate in regulating neointima formation. The expression of different NOTCH members is regulated in a temporal manner after vascular injury ${ }^{87-89}$. It appears that NOTCH1, 2 and 3 together with Herp-1 and Herp- 2 are downregulated in the first 7 days after balloon injury, after which at least NOTCH 1 and 4 together with Jagged- 1 and -2 are upregulated at the leading edges of the regenerating endothelium ${ }^{88}$. Loss of function studies with general as well as smooth muscle cell specific NOTCH1 heterozygous mice support the notion that $\mathrm{NOTCH}$ activation is required for neointima formation ${ }^{90}$. Accordingly, in Hey2-/- mice ${ }^{91}$ VSMC proliferation was reduced resulting in deminished neointima formation. Consistently, gain of function studies with overexpression of HRT1 (also named Hey1/HERP2/Hesr-1/CHF2) ${ }^{92}$ or constitutive expression of Notch 1 and $3^{93}$ induced SMCs proliferation and migration capacity with enhanced neointima formation as a result.

An intact and functional endothelial layer appears to maintain VSMC in a quiescent state $^{94,95}$. Although several mechanisms have been reported, including NO (Nitric Oxide) and heparin-like substances, definitive proof for a particular mechanism has not been established yet. The NOTCH pathway might be a suitable intercellular signaling to mediate this mechanism. Under certain conditions, Delta and Jagged can be cleaved from the cell membrane generating a soluble antagonist of NOTCH activity $^{96}$. Duarte et al $^{48}$ have shown that thrombin mediates the proteolytic release of Jagged1, which subsequently inhibits NOTCH signaling. SMC migration and proliferation are positively regulated by $\mathrm{NOTCH}$ signaling and are induced following vascular injury. Lindner et $\mathrm{al}^{88}$ have shown that soluble Jagged decreased cell-matrix interaction, focal adhesion formation and migration in NIH3T3 cells. Altogether these observations suggest a possible therapeutic application for soluble ligands to limit the response to vascular injury, by interfering with NOTCH signaling and SMCS proliferation and migration. 


\section{Chapter Outline}

The aim of this thesis was to study the NOTCH signaling pathway in vascular biology. First we investigated the molecular mechanisms of VEGF-NOTCH signaling interaction in ECS and the role of ADAMs in this process. Furthermore, we aimed to study the effect of VEGF-NOTCH signaling on coronary arteries development and arterial specification. Finally, we studied the effect of a soluble NOTCH ligand, sJag1, on SMCs behaviour and neointima formation following vascular injury.

Chapter 1 provides background information on the multiple roles of $\mathrm{NOTCH}$ signaling in blood vessels. The interaction between NOTCH and VEGF signaling in regulating artery/vein identity and sprouting of endothelial cells is introduced. The role of NOTCH in regulating VSMC differentiation in normal or injured vessels is outlined.

Chapter 2 provides a detailed molecular analysis of the VEGF and NOTCH signaling interaction. We demonstrated that VEGFR-2-induced DLL4 expression is NOTCH mediated. Accordingly, VEGF also induced NOTCH signaling activation. Moreover, DLL4 induced its own expression in a NOTCH-dependent manner unrelated to VEGFsignaling, indicating a feed-forward mechanism downstream of NOTCH in ECs.

Chapter 3 shows the functional interaction between the VEGFR-2 pathway and ADAM-10, a metalloproteinase involved in NOTCH processing and activation. VEGF induces ADAM-10 expression and activation in ECs, increasing vascular permeability and facilitate ECs migration. Furthermore, ADAM-10 expression in atherosclerosis lesions is associated with plaque progression and neovascularization.

Chapter 4 reports on the effect of altered VEGF-signaling in Vegf120/120 mouse embryos on coronary development. Mutant coronary artery endothelium showed decrease expression of arterial marker such as NOTCH-1, DLL4 and EphrinB2 and an increase of venous marker expression, whereas the venous endothelium showed opposite effects. Coronary arteriogenesis was also anomalous in mutant embryos with decreased arterial and increased venous medial development.

Chapter 5 describes the role of the soluble NOTCH ligand Jag1 in inhibiting neointima formation in a rat injured carotid artery model. Soluble-Jagged reduced SMC proliferation and migration following vascular injury, by interfering with NOTCH signaling activation and NOTCH target gene Herp2 expression.

Chapter 6 discusses the regulation of the NOTCH pathway and ADAM-10 activity by VEGF signaling together with the existence of a NOTCH feed-forward mechanism in ECs; the role of VEGF and NOTCH signaling in coronary endothelial differentiation and maturation; the negative effect of sJag1 on neointima formation. 


\section{References}

1 Conway, E. M., Collen, D. and Carmeliet, P., Molecular mechanisms of blood vessel growth, Cardiovasc Res, 2001, 49: 507-521.

2 Jain, R. K., Molecular regulation of vessel maturation, Nat Med, 2003, 9: 685-693.

3 Djonov, V., Baum, O. and Burri, P. H., Vascular remodeling by intussusceptive angiogenesis, Cell Tissue Res, 2003, 314: 107-117.

4 Burri, P. H. and Djonov, V., Intussusceptive angiogenesis--the alternative to capillary sprouting, $\mathrm{Mol}$ Aspects Med, 2002, 23: S1-27.

5 Artavanis-Tsakonas, S., Rand, M. D. and Lake, R. J., Notch signaling: cell fate control and signal integration in development, Science, 1999, 284: 770-776.

6 Krebs, L. T., Xue, Y., Norton, C. R., Shutter, J. R., Maguire, M., Sundberg, J. P., Gallahan, D., Closson, V., Kitajewski, J., Callahan, R., Smith, G. H., Stark, K. L. and Gridley, T., Notch signaling is essential for vascular morphogenesis in mice, Genes Dev, 2000, 14: 1343-1352.

7 Xue, Y., Gao, X., Lindsell, C. E., Norton, C. R., Chang, B., Hicks, C., Gendron-Maguire, M., Rand, E. B., Weinmaster, G. and Gridley, T., Embryonic lethality and vascular defects in mice lacking the Notch ligand Jagged1, Hum Mol Genet, 1999, 8: 723-730.

8 Huppert, S. S., Le, A., Schroeter, E. H., Mumm, J. S., Saxena, M. T., Milner, L. A. and Kopan, R., Embryonic lethality in mice homozygous for a processing-deficient allele of Notch1, Nature, 2000, 405: 966-970.

9 Hrabe de Angelis, M., Mclntyre, J., 2nd and Gossler, A., Maintenance of somite borders in mice requires the Delta homologue DII1, Nature, 1997, 386: 717-721.

10 McCright, B., Gao, X., Shen, L., Lozier, J., Lan, Y., Maguire, M., Herzlinger, D., Weinmaster, G., Jiang, R. and Gridley, T., Defects in development of the kidney, heart and eye vasculature in mice homozygous for a hypomorphic Notch2 mutation, Development, 2001, 128: 491-502.

11 Duarte, A., Hirashima, M., Benedito, R., Trindade, A., Diniz, P., Bekman, E., Costa, L., Henrique, D. and Rossant, J., Dosage-sensitive requirement for mouse Dll4 in artery development, Genes Dev, 2004, 18: 2474-2478.

12 Gale, N. W., Dominguez, M. G., Noguera, I., Pan, L., Hughes, V., Valenzuela, D. M., Murphy, A. J., Adams, N. C., Lin, H. C., Holash, J., Thurston, G. and Yancopoulos, G. D., Haploinsufficiency of deltalike 4 ligand results in embryonic lethality due to major defects in arterial and vascular development, Proc Natl Acad Sci U S A, 2004, 101: 15949-15954.

13 Krebs, L. T., Shutter, J. R., Tanigaki, K., Honjo, T., Stark, K. L. and Gridley, T., Haploinsufficient lethality and formation of arteriovenous malformations in Notch pathway mutants, Genes Dev, 2004, 18: 2469-2473.

14 Warthen, D. M., Moore, E. C., Kamath, B. M., Morrissette, J. J., Sanchez, P., Piccoli, D. A., Krantz, I. D. and Spinner, N. B., Jagged1 (JAG1) mutations in Alagille syndrome: increasing the mutation detection rate, Hum Mutat, 2006, 27: 436-443.

15 Joutel, A., Corpechot, C., Ducros, A., Vahedi, K., Chabriat, H., Mouton, P., Alamowitch, S., Domenga, V., Cecillion, M., Marechal, E., Maciazek, J., Vayssiere, C., Cruaud, C., Cabanis, E. A., Ruchoux, M. M., Weissenbach, J., Bach, J. F., Bousser, M. G. and Tournier-Lasserve, E., Notch3 mutations in CADASIL, a hereditary adult-onset condition causing stroke and dementia, Nature, 1996, 383: 707-710.

16 Kalaria, R. N., Viitanen, M., Kalimo, H., Dichgans, M. and Tabira, T., The pathogenesis of CADASIL: an update, J Neurol Sci, 2004, 226: 35-39.

17 Weinmaster, G., Notch signal transduction: a real rip and more, Curr Opin Genet Dev, 2000, 10: 363369.

18 Bray, S. J., Notch signalling: a simple pathway becomes complex, Nat Rev Mol Cell Biol, 2006, 7: 678689.

19 Hurlbut, G. D., Kankel, M. W., Lake, R. J. and Artavanis-Tsakonas, S., Crossing paths with Notch in the hyper-network, Curr Opin Cell Biol, 2007, 19: 166-175.

20 Kurooka, H., Kuroda, K. and Honjo, T., Roles of the ankyrin repeats and C-terminal region of the mouse notch1 intracellular region, Nucleic Acids Res, 1998, 26: 5448-5455. 
21 Iso, T., Kedes, L. and Hamamori, Y., HES and HERP families: multiple effectors of the Notch signaling pathway, J Cell Physiol, 2003, 194: 237-255.

22 Lai, E. C., Keeping a good pathway down: transcriptional repression of Notch pathway target genes by CSL proteins, EMBO Rep, 2002, 3: 840-845.

23 Fischer, A. and Gessler, M., Hey genes in cardiovascular development, Trends Cardiovasc Med, 2003, 13: 221-226.

24 Ehebauer, M., Hayward, P. and Martinez-Arias, A., Notch signaling pathway, Sci STKE, 2006, 2006: cm7.

25 Siekmann, A. F., Covassin, L. and Lawson, N. D., Modulation of VEGF signalling output by the Notch pathway, Bioessays, 2008, 30: 303-313.

26 Jakobsson, L., Bentley, K. and Gerhardt, H., VEGFRs and Notch: a dynamic collaboration in vascular patterning, Biochem Soc Trans, 2009, 37: 1233-1236.

27 Ferrara, N., Gerber, H. P. and LeCouter, J., The biology of VEGF and its receptors, Nat Med, 2003, 9: 669-676.

28 Olsson, A. K., Dimberg, A., Kreuger, J. and Claesson-Welsh, L., VEGF receptor signalling - in control of vascular function, Nat Rev Mol Cell Biol, 2006, 7: 359-371.

29 Stuttfeld, E. and Ballmer-Hofer, K., Structure and function of VEGF receptors, IUBMB Life, 2009, 61: 915-922.

30 Neufeld, G., Cohen, T., Gengrinovitch, S. and Poltorak, Z., Vascular endothelial growth factor (VEGF) and its receptors, Faseb J, 1999, 13: 9-22.

31 Carmeliet, P., Moons, L., Luttun, A., Vincenti, V., Compernolle, V., De Mol, M., Wu, Y., Bono, F., Devy, L., Beck, H., Scholz, D., Acker, T., DiPalma, T., Dewerchin, M., Noel, A., Stalmans, I., Barra, A., Blacher, S., Vandendriessche, T., Ponten, A., Eriksson, U., Plate, K. H., Foidart, J. M., Schaper, W., CharnockJones, D. S., Hicklin, D. J., Herbert, J. M., Collen, D. and Persico, M. G., Synergism between vascular endothelial growth factor and placental growth factor contributes to angiogenesis and plasma extravasation in pathological conditions, Nat Med, 2001, 7: 575-583.

32 Carmeliet, P., Ferreira, V., Breier, G., Pollefeyt, S., Kieckens, L., Gertsenstein, M., Fahrig, M., Vandenhoeck, A., Harpal, K., Eberhardt, C., Declercq, C., Pawling, J., Moons, L., Collen, D., Risau, W. and Nagy, A., Abnormal blood vessel development and lethality in embryos lacking a single VEGF allele, Nature, 1996, 380: 435-439.

33 Ferrara, N., Carver-Moore, K., Chen, H., Dowd, M., Lu, L., O’Shea, K. S., Powell-Braxton, L., Hillan, K. J. and Moore, M. W., Heterozygous embryonic lethality induced by targeted inactivation of the VEGF gene, Nature, 1996, 380: 439-442.

34 Miquerol, L., Langille, B. L. and Nagy, A., Embryonic development is disrupted by modest increases in vascular endothelial growth factor gene expression, Development, 2000, 127: 3941-3946.

35 Takahashi, H. and Shibuya, M., The vascular endothelial growth factor (VEGF)/VEGF receptor system and its role under physiological and pathological conditions, Clin Sci (Lond), 2005, 109: 227-241.

36 Waltenberger, J., Claesson-Welsh, L., Siegbahn, A., Shibuya, M. and Heldin, C. H., Different signal transduction properties of KDR and Flt1, two receptors for vascular endothelial growth factor, $J$ Biol Chem, 1994, 269: 26988-26995.

37 Shalaby, F., Rossant, J., Yamaguchi, T. P., Gertsenstein, M., Wu, X. F., Breitman, M. L. and Schuh, A. C., Failure of blood-island formation and vasculogenesis in Flk-1-deficient mice, Nature, 1995, 376: 62-66.

38 Meyer, R. D., Mohammadi, M. and Rahimi, N., A single amino acid substitution in the activation loop defines the decoy characteristic of VEGFR-1/FLT-1, J Biol Chem, 2006, 281: 867-875.

39 Hiratsuka, S., Minowa, O., Kuno, J., Noda, T. and Shibuya, M., Flt-1 lacking the tyrosine kinase domain is sufficient for normal development and angiogenesis in mice, Proc Natl Acad Sci U S A, 1998, 95: 9349-9354.

40 Fong, G. H., Rossant, J., Gertsenstein, M. and Breitman, M. L., Role of the Flt-1 receptor tyrosine kinase in regulating the assembly of vascular endothelium, Nature, 1995, 376: 66-70. 
41 Clauss, M., Weich, H., Breier, G., Knies, U., Rockl, W., Waltenberger, J. and Risau, W., The vascular endothelial growth factor receptor Flt-1 mediates biological activities. Implications for a functional role of placenta growth factor in monocyte activation and chemotaxis, J Biol Chem, 1996, 271: 17629-17634.

42 Tchaikovski, V., Fellbrich, G. and Waltenberger, J., The molecular basis of VEGFR-1 signal transduction pathways in primary human monocytes, Arterioscler Thromb Vasc Biol, 2008, 28: 322 328.

43 Lawson, N. D., Vogel, A. M. and Weinstein, B. M., sonic hedgehog and vascular endothelial growth factor act upstream of the Notch pathway during arterial endothelial differentiation, Dev Cell, 2002, 3: 127-136.

44 Lawson, N. D., Scheer, N., Pham, V. N., Kim, C. H., Chitnis, A. B., Campos-Ortega, J. A. and Weinstein, B. M., Notch signaling is required for arterial-venous differentiation during embryonic vascular development, Development, 2001, 128: 3675-3683.

45 Visconti, R. P., Richardson, C. D. and Sato, T. N., Orchestration of angiogenesis and arteriovenous contribution by angiopoietins and vascular endothelial growth factor (VEGF), Proc Natl Acad Sci U S A, 2002, 99: 8219-8224.

46 Mukouyama, Y. S., Shin, D., Britsch, S., Taniguchi, M. and Anderson, D. J., Sensory nerves determine the pattern of arterial differentiation and blood vessel branching in the skin, Cell, 2002, 109: 693705.

47 Mukouyama, Y. S., Gerber, H. P., Ferrara, N., Gu, C. and Anderson, D. J., Peripheral nerve-derived VEGF promotes arterial differentiation via neuropilin 1-mediated positive feedback, Development, 2005, 132: 941-952.

48 Duarte, M., Kolev, V., Kacer, D., Mouta-Bellum, C., Soldi, R., Graziani, I., Kirov, A., Friesel, R., Liaw, L., Small, D., Verdi, J., Maciag, T. and Prudovsky, I., Novel cross-talk between three cardiovascular regulators: thrombin cleavage fragment of Jagged1 induces fibroblast growth factor 1 expression and release, Mol Biol Cell, 2008, 19: 4863-4874.

49 Fischer, A., Schumacher, N., Maier, M., Sendtner, M. and Gessler, M., The Notch target genes Hey1 and Hey2 are required for embryonic vascular development, Genes Dev, 2004, 18: 901-911.

50 Kokubo, H., Miyagawa-Tomita, S., Nakazawa, M., Saga, Y. and Johnson, R. L., Mouse hesr1 and hesr2 genes are redundantly required to mediate Notch signaling in the developing cardiovascular system, Dev Biol, 2005, 278: 301-309.

51 Iso, T., Maeno, T., Oike, Y., Yamazaki, M., Doi, H., Arai, M. and Kurabayashi, M., Dll4-selective Notch signaling induces ephrinB2 gene expression in endothelial cells, Biochem Biophys Res Commun, 2006, 341: 708-714.

52 Carlson, T. R., Yan, Y., Wu, X., Lam, M. T., Tang, G. L., Beverly, L. J., Messina, L. M., Capobianco, A. J., Werb, Z. and Wang, R., Endothelial expression of constitutively active Notch4 elicits reversible arteriovenous malformations in adult mice, Proc Natl Acad Sci U S A, 2005, 102: 9884-9889.

53 Liu, Z. J., Shirakawa, T., Li, Y., Soma, A., Oka, M., Dotto, G. P., Fairman, R. M., Velazquez, O. C. and Herlyn, M., Regulation of Notch1 and Dll4 by vascular endothelial growth factor in arterial endothelial cells: implications for modulating arteriogenesis and angiogenesis, Mol Cell Biol, 2003, 23: 14-25.

54 Lanner, F., Sohl, M. and Farnebo, F., Functional arterial and venous fate is determined by graded VEGF signaling and notch status during embryonic stem cell differentiation, Arterioscler Thromb Vasc Biol, 2007, 27: 487-493.

55 Zhang, G., Zhou, J., Fan, Q., Zheng, Z., Zhang, F., Liu, X. and Hu, S., Arterial-venous endothelial cell fate is related to vascular endothelial growth factor and Notch status during human bone mesenchymal stem cell differentiation, FEBS Lett, 2008, 582: 2957-2964.

56 You, L. R., Lin, F. J., Lee, C. T., DeMayo, F. J., Tsai, M. J. and Tsai, S. Y., Suppression of Notch signalling by the COUP-TFII transcription factor regulates vein identity, Nature, 2005, 435: 98-104. 
57 Masumura, T., Yamamoto, K., Shimizu, N., Obi, S. and Ando, J., Shear stress increases expression of the arterial endothelial marker ephrinB2 in murine ES cells via the VEGF-Notch signaling pathways, Arterioscler Thromb Vasc Biol, 2009, 29: 2125-2131.

58 Nakamura, K., Martin, K. C., Jackson, J. K., Beppu, K., Woo, C. W. and Thiele, C. J., Brain-derived neurotrophic factor activation of TrkB induces vascular endothelial growth factor expression via hypoxia-inducible factor-1alpha in neuroblastoma cells, Cancer Res, 2006, 66: 4249-4255.

59 Pufe, T., Lemke, A., Kurz, B., Petersen, W., Tillmann, B., Grodzinsky, A. J. and Mentlein, R., Mechanical overload induces VEGF in cartilage discs via hypoxia-inducible factor, Am J Pathol, 2004, 164: 185-192.

60 Fukuda, R., Kelly, B. and Semenza, G. L., Vascular endothelial growth factor gene expression in colon cancer cells exposed to prostaglandin E2 is mediated by hypoxia-inducible factor 1, Cancer Res, 2003, 63: 2330-2334.

61 Rissanen, T. T., Vajanto, I., Hiltunen, M. O., Rutanen, J., Kettunen, M. I., Niemi, M., Leppanen, P., Turunen, M. P., Markkanen, J. E., Arve, K., Alhava, E., Kauppinen, R. A. and Yla-Herttuala, S., Expression of vascular endothelial growth factor and vascular endothelial growth factor receptor-2 (KDR/Flk-1) in ischemic skeletal muscle and its regeneration, Am J Pathol, 2002, 160: 1393-1403.

62 Diez, H., Fischer, A., Winkler, A., Hu, C. J., Hatzopoulos, A. K., Breier, G. and Gessler, M., Hypoxiamediated activation of Dll4-Notch-Hey2 signaling in endothelial progenitor cells and adoption of arterial cell fate, Exp Cell Res, 2007, 313: 1-9.

63 Gerhardt, H., Golding, M., Fruttiger, M., Ruhrberg, C., Lundkvist, A., Abramsson, A., Jeltsch, M., Mitchell, C., Alitalo, K., Shima, D. and Betsholtz, C., VEGF guides angiogenic sprouting utilizing endothelial tip cell filopodia, J Cell Biol, 2003, 161: 1163-1177.

64 Gerhardt, H., Ruhrberg, C., Abramsson, A., Fujisawa, H., Shima, D. and Betsholtz, C., Neuropilin-1 is required for endothelial tip cell guidance in the developing central nervous system, Dev Dyn, 2004, 231: 503-509.

65 Jakobsson, L., Franco, C. A., Bentley, K., Collins, R. T., Ponsioen, B., Aspalter, I. M., Rosewell, I., Busse, M., Thurston, G., Medvinsky, A., Schulte-Merker, S. and Gerhardt, H., Endothelial cells dynamically compete for the tip cell position during angiogenic sprouting, Nat Cell Biol, 12: 943-953.

66 Suchting, S., Freitas, C., le Noble, F., Benedito, R., Breant, C., Duarte, A. and Eichmann, A., The Notch ligand Delta-like 4 negatively regulates endothelial tip cell formation and vessel branching, Proc Natl Acad Sci U S A, 2007, 104: 3225-3230.

67 Suchting, S., Freitas, C., le Noble, F., Benedito, R., Breant, C., Duarte, A. and Eichmann, A., Negative regulators of vessel patterning, Novartis Found Symp, 2007, 283: 77-80; discussion 80-76, 238-241.

68 Hellstrom, M., Phng, L. K., Hofmann, J. J., Wallgard, E., Coultas, L., Lindblom, P., Alva, J., Nilsson, A. K., Karlsson, L., Gaiano, N., Yoon, K., Rossant, J., Iruela-Arispe, M. L., Kalen, M., Gerhardt, H. and Betsholtz, C., Dll4 signalling through Notch1 regulates formation of tip cells during angiogenesis, Nature, 2007, 445: 776-780.

69 Lobov, I. B., Renard, R. A., Papadopoulos, N., Gale, N. W., Thurston, G., Yancopoulos, G. D. and Wiegand, S. J., Delta-like ligand 4 (DII4) is induced by VEGF as a negative regulator of angiogenic sprouting, Proc Natl Acad Sci U S A, 2007, 104: 3219-3224.

70 Ridgway, J., Zhang, G., Wu, Y., Stawicki, S., Liang, W. C., Chanthery, Y., Kowalski, J., Watts, R. J., Callahan, C., Kasman, I., Singh, M., Chien, M., Tan, C., Hongo, J. A., de Sauvage, F., Plowman, G. and Yan, M., Inhibition of Dll4 signalling inhibits tumour growth by deregulating angiogenesis, Nature, 2006, 444: 1083-1087.

71 Leslie, J. D., Ariza-McNaughton, L., Bermange, A. L., McAdow, R., Johnson, S. L. and Lewis, J., Endothelial signalling by the Notch ligand Delta-like 4 restricts angiogenesis, Development, 2007, 134: 839-844.

72 Siekmann, A. F. and Lawson, N. D., Notch signalling limits angiogenic cell behaviour in developing zebrafish arteries, Nature, 2007, 445: 781-784. 
73 Noguera-Troise, I., Daly, C., Papadopoulos, N. J., Coetzee, S., Boland, P., Gale, N. W., Lin, H. C., Yancopoulos, G. D. and Thurston, G., Blockade of Dll4 inhibits tumour growth by promoting nonproductive angiogenesis, Nature, 2006, 444: 1032-1037.

74 Scehnet, J. S., Jiang, W., Kumar, S. R., Krasnoperov, V., Trindade, A., Benedito, R., Djokovic, D., Borges, C., Ley, E. J., Duarte, A. and Gill, P. S., Inhibition of Dll4-mediated signaling induces proliferation of immature vessels and results in poor tissue perfusion, Blood, 2007, 109: 4753-4760.

75 Domenga, V., Fardoux, P., Lacombe, P., Monet, M., Maciazek, J., Krebs, L. T., Klonjkowski, B., Berrou, E., Mericskay, M., Li, Z., Tournier-Lasserve, E., Gridley, T. and Joutel, A., Notch3 is required for arterial identity and maturation of vascular smooth muscle cells, Genes Dev, 2004, 18: 2730-2735.

76 van der Loop, F. T., Gabbiani, G., Kohnen, G., Ramaekers, F. C. and van Eys, G. J., Differentiation of smooth muscle cells in human blood vessels as defined by smoothelin, a novel marker for the contractile phenotype, Arterioscler Thromb Vasc Biol, 1997, 17: 665-671.

77 Moessler, H., Mericskay, M., Li, Z., Nagl, S., Paulin, D. and Small, J. V., The SM 22 promoter directs tissue-specific expression in arterial but not in venous or visceral smooth muscle cells in transgenic mice, Development, 1996, 122: 2415-2425.

78 Doi, H., Iso, T., Sato, H., Yamazaki, M., Matsui, H., Tanaka, T., Manabe, I., Arai, M., Nagai, R. and Kurabayashi, M., Jagged1-selective notch signaling induces smooth muscle differentiation via a RBPJkappa-dependent pathway, J Biol Chem, 2006, 281: 28555-28564.

79 Liu, H., Kennard, S. and Lilly, B., NOTCH3 expression is induced in mural cells through an autoregulatory loop that requires endothelial-expressed JAGGED1, Circ Res, 2009, 104: 466-475.

80 Noseda, M., Fu, Y., Niessen, K., Wong, F., Chang, L., McLean, G. and Karsan, A., Smooth Muscle alphaactin is a direct target of Notch/CSL, Circ Res, 2006, 98: 1468-1470.

81 High, F. A., Lu, M. M., Pear, W. S., Loomes, K. M., Kaestner, K. H. and Epstein, J. A., Endothelial expression of the Notch ligand Jagged1 is required for vascular smooth muscle development, Proc Natl Acad Sci U S A, 2008, 105: 1955-1959.

82 Doi, H., Iso, T., Yamazaki, M., Akiyama, H., Kanai, H., Sato, H., Kawai-Kowase, K., Tanaka, T., Maeno, T., Okamoto, E., Arai, M., Kedes, L. and Kurabayashi, M., HERP1 inhibits myocardin-induced vascular smooth muscle cell differentiation by interfering with SRF binding to CArG box, Arterioscler Thromb Vasc Biol, 2005, 25: 2328-2334.

83 Tang, Y., Urs, S. and Liaw, L., Hairy-related transcription factors inhibit Notch-induced smooth muscle alpha-actin expression by interfering with Notch intracellular domain/CBF-1 complex interaction with the CBF-1-binding site, Circ Res, 2008, 102: 661-668.

84 Proweller, A., Pear, W. S. and Parmacek, M. S., Notch signaling represses myocardin-induced smooth muscle cell differentiation, J Biol Chem, 2005, 280: 8994-9004.

85 Limbourg, A., Ploom, M., Elligsen, D., Sorensen, I., Ziegelhoeffer, T., Gossler, A., Drexler, H. and Limbourg, F. P., Notch ligand Delta-like 1 is essential for postnatal arteriogenesis, Circ Res, 2007, 100: 363-371.

86 Takeshita, K., Satoh, M., li, M., Silver, M., Limbourg, F. P., Mukai, Y., Rikitake, Y., Radtke, F., Gridley, T., Losordo, D. W. and Liao, J. K., Critical role of endothelial Notch1 signaling in postnatal angiogenesis, Circ Res, 2007, 100: 70-78.

87 Campos, A. H., Wang, W., Pollman, M. J. and Gibbons, G. H., Determinants of Notch-3 receptor expression and signaling in vascular smooth muscle cells: implications in cell-cycle regulation, Circ Res, 2002, 91: 999-1006.

88 Lindner, V., Booth, C., Prudovsky, I., Small, D., Maciag, T. and Liaw, L., Members of the Jagged/Notch gene families are expressed in injured arteries and regulate cell phenotype via alterations in cell matrix and cell-cell interaction, Am J Pathol, 2001, 159: 875-883.

89 Wang, W., Campos, A. H., Prince, C. Z., Mou, Y. and Pollman, M. J., Coordinate Notch3-hairy-related transcription factor pathway regulation in response to arterial injury. Mediator role of plateletderived growth factor and ERK, J Biol Chem, 2002, 277: 23165-23171. 
90 Li, Y., Takeshita, K., Liu, P. Y., Satoh, M., Oyama, N., Mukai, Y., Chin, M. T., Krebs, L., Kotlikoff, M. I., Radtke, F., Gridley, T. and Liao, J. K., Smooth muscle Notch1 mediates neointimal formation after vascular injury, Circulation, 2009, 119: 2686-2692.

91 Sakata, Y., Xiang, F., Chen, Z., Kiriyama, Y., Kamei, C. N., Simon, D. I. and Chin, M. T., Transcription factor CHF1/Hey2 regulates neointimal formation in vivo and vascular smooth muscle proliferation and migration in vitro, Arterioscler Thromb Vasc Biol, 2004, 24: 2069-2074.

92 Wang, W., Prince, C. Z., Hu, X. and Pollman, M. J., HRT1 modulates vascular smooth muscle cell proliferation and apoptosis, Biochem Biophys Res Commun, 2003, 308: 596-601.

93 Sweeney, C., Morrow, D., Birney, Y. A., Coyle, S., Hennessy, C., Scheller, A., Cummins, P. M., Walls, D., Redmond, E. M. and Cahill, P. A., Notch 1 and 3 receptor signaling modulates vascular smooth muscle cell growth, apoptosis, and migration via a CBF-1/RBP-Jk dependent pathway, Faseb J, 2004, 18: 1421-1423.

94 Lindner, V., Expression of platelet-derived growth factor ligands and receptors by rat aortic endothelium in vivo, Pathobiology, 1995, 63: 257-264.

95 Lindner, V. and Reidy, M. A., Platelet-derived growth factor ligand and receptor expression by large vessel endothelium in vivo, Am J Pathol, 1995, 146: 1488-1497.

96 Qi, H., Rand, M. D., Wu, X., Sestan, N., Wang, W., Rakic, P., Xu, T. and Artavanis-Tsakonas, S., Processing of the notch ligand delta by the metalloprotease Kuzbanian, Science, 1999, 283: 91-94. 


\title{
Chapter 2
}

\section{Feed-forward signaling by a membrane} bound ligand receptor circuit: The case of NOTCH-DELTA LIKE 4 in endothelial cells

\author{
Vincenza Caolo ${ }^{1}$, Nynke M.S. van den Akker ${ }^{2}$, Sanne Verbruggen ${ }^{1}$, Marjo M.P.C. \\ Donners ${ }^{3}$, Geertje Swennen ${ }^{1}$, Henny Schulten ${ }^{1}$, Johannes Waltenberger ${ }^{2}$, Mark J. \\ Post $^{1}$, Daniel G.M. Molin ${ }^{1}$ \\ ${ }^{1}$ Dept. of Physiology, ${ }^{2}$ Dept. of Cardiology, ${ }^{3}$ Dept. of Molecular Genetics, \\ Cardiovascular Research Institute Maastricht (CARIM), Maastricht University, the \\ Netherlands
}

Modified after Journal of Biological Chemistry, 2010;285:40681-89. 



\begin{abstract}
The Delta like-4 (DLL4) ligand belongs to the highly conserved NOTCH-family and is specifically expressed in the endothelium. DLL4 regulates crucial processes in vascular growth, including endothelial cell (EC) sprouting and arterial specification. Its expression is increased by VEGF-A. In the present study we show that VEGFinduced DLL4 expression depends on NOTCH activation. VEGF-induced DLL4 expression was prevented by the blockage of $\mathrm{NOTCH}$-signaling with $\gamma$-secretase or ADAMs inhibitors in Human Cardiac MicroVascular ECs. Similar to VEGF-A, recombinant DLL4 itself stimulated NOTCH-signaling and resulted in up-regulation of DLL4, suggesting a positive feed-forward mechanism. These effects were abrogated by $\mathrm{NOTCH}$ inhibitors, but not by inhibition of VEGF-signaling. NOTCH activation alone suffices to induce DLL4 expression as illustrated by the positive effect of Notch Intracellular Domain (NICD) -1 or -4 overexpression. To discriminate between NICD/RBP-Jk and FOXC2 regulated DLL4 expression, DLL4 promoteractivity was assessed in promoter deletion experiments. NICD induced promoter activity was dependent on RBP-Jk site but independent of the FOXC2 binding site. Accordingly, constitutively active FOXC2 did not affect DLL4 expression. The notion that the positive feed-forward mechanism might propagate $\mathrm{NOTCH}$-activation to neighboring ECs was supported by our observation that DLL4-eGFP transfected ECS induced DLL4 expression in non-transfected cells in their vicinity. In summary, our data provide evidence for a mechanism by which VEGF or ligand-induced NOTCHsignaling up-regulates DLL4 through a positive feed-forward mechanism. By this mechanism DLL4 could propagate its own expression and enable synchronization of NOTCH expression and signaling between ECs.
\end{abstract}




\section{Introduction}

The NOTCH family encompasses a fundamental signaling pathway involving four receptors (NOTCH-1, $-2,-3,-4$ ) and, in vertebrates, five cognate ligands (DLL-1, -3, -4 and JAGGED-1, -2). Cell-cell contact is a prerequisite for NOTCH signaling as all members are membrane bound. The first activation step upon receptor-ligand interaction involves cleavage of the extracellular domain of the NOTCH receptor by A Disintegrin And Metalloprotease (ADAM). Subsequently, the $\gamma$-secretase complex instigates a second proteolytic cleavage resulting in the release of the NOTCH intracellular domain (NICD) into the cytoplasm. Next, the NICD translocates to the nucleus, where it associates with the Recombinant Signal binding protein for Immunoglobulin kappa J region (RBP-Jk or CSL, CBF1/RBPJk, $\underline{\text { u }}(\mathrm{H})$, Lag1) transcription factor to initiate the transcription of its downstream targets basic Helix-Loop-Helix proteins Hairy/Enhancer of Split (HES) and Hairy Related Transcription factors (HRT, HEY, HERP) ${ }^{1}$.

Among the ligands, DLL4 is specifically expressed in the arterial endothelium and plays a major role during embryonic vascular development. DII4 haploinsufficiency is embryonically lethal due to severe vascular abnormalities related to a loss of arterial and venous specification ${ }^{2-7}$. DLL4 overexpression on the other hand induces arterialization even in the venous compartment that typically lacks NOTCH expression $^{8}$.

Notch signaling is important for the development of endothelial cell sprouting. In this process VEGF induces the formation of new sprouts, whereas DLL4 signaling appears to reduce their formation ${ }^{9,10}$. Since DLL4 is upregulated by VEGF ${ }^{11}$ this signaling pathway might serve as a negative feedback loop with respect to endothelial sprouting. This feedback could be bidirectional as NOTCH reduces VEGF responsiveness through downregulation of VEGFR-2 ${ }^{12}$ and subsequently, suppresses the sprouting phenotype in the adjacent stalk cell, thus limiting the number of sprouts. Accordingly, loss of DLL4 function promotes excessive endothelial cell sprouting and endothelial cell migration ${ }^{13}$. VEGF-A has also been reported to induce the activation of ADAM-10 and $-17^{14}$, which are the protease required for the first step in NOTCH activation. This interrelation between VEGF-A and Notch signaling suggests that VEGF not only induces the expression of DLL4 but also stimulates NOTCH signaling. However, alternative pathways have been suggested for VEGFinduced DLL4 expression. Upregulation of DLL4 by VEGF has been considered the result of activated FOXC (Forkhead box C) transcription factors by a PI3K and ERK/MAPK dependent pathway ${ }^{15}$. FOXC2 has been shown to activate the DLL4 and HEY2 promoters, which both contain a forkhead binding element $(F B E)^{15,16}$. In particular, FOXC2 functionally interacts with RBP-Jk and NICD to activate the HEY2 promoter $^{15}$. Similarly to HEY2, the DLL4 promoter presents with multiple RBP-Jk binding sites ${ }^{17}$, suggesting that NOTCH might regulate DLL4 expression as well. Altogether these observations prompted us to speculate that $\mathrm{NOTCH}$-signaling 
could play a role in up-regulating the expression of its own ligand DLL4, which in turn would activate NOTCH signal transduction, thus creating a positive feedforward signaling that propagates the activation to adjacent cells.

In the present study we show that in endothelial cells, VEGF-induced DLL4 upregulation is NOTCH activation dependent. VEGF positively affects DLL4 expression and NOTCH signaling mainly by activating VEGFR-2, whereas inhibition of NOTCH signaling abrogated VEGF-induced DLL4 expression. Consistently, immobilized recombinant DLL4 also induced endogenous DLL4 expression in a NOTCHdependent manner unrelated to VEGF-signaling, indicating a feed-forward mechanism downstream of NOTCH. The increase in DLL4, along with transactivation of the DLL4 promoter by NICD-1 and NICD-4 confirmed the role of NOTCH signaling in this process, further suggesting that NOTCH activation is sufficient for DLL4 induction. In this endothelial system, we found no evidence for FOXC2 dependent DLL4 expression by VEGF. Finally, we show that DLL4 transfected endothelial cells were able to increase DLL4 expression of surrounding cells providing a mechanism by which NOTCH signaling is distributed between communicating endothelial cells.

\section{Experimental Procedures}

\section{Reagents}

Human Cardiac Microvascular Endothelial Cells (HCMvECs) (Lonza, Vervies, Belgium) were cultured in EGM-2MV, with 5\% FBS (FBS; Lonza). Recombinant VEGF- $\mathrm{A}_{165}$ was purchased from RELIATech GmbH (Brauschweig, Germany); DLL4 (rDLL4) extracellular domain was from R\&D System (Abingdon, Oxfordshire, UK); L685,458 ( $\gamma$-secretase inhibitor) from Calbiochem (Notthingam, UK); IMC-1121b (human VEGFR-2 inhibitor) and IMC-18F1 (human VEGFR-1 inhibitor) ${ }^{18,19}$ were both kindly provided by ImClone Systems Corporation (New York, NY, USA). ADAM inhibitors GI250423X (ADAM-10 inhibitors) and GW28026X (ADAM-10/ADAM-17 inhibitor) ${ }^{20}$ were both kindly provided by GlaxoSmithKline.

\section{Cell Culture and Cell Treatment}

HCMvECs were cultured according to manufacturer's protocol (Lonza). Cells were cultured in EGM-2MV medium without basic Fibroblast Growth Factor (bFGF) and VEGF-A $A_{165}$. Cells were stimulated for 24 hours with $100 \mathrm{ng} / \mathrm{mL}$ of human recombinant VEGF- $A_{165}$. For rDll4 stimulation experiments HCMvECs were grown for 48 hours on $0.2 \%$ gelatin coated plates containing $1 \mu \mathrm{g} / \mathrm{mL}$ rD\|l4 or BSA as control ${ }^{21}$.

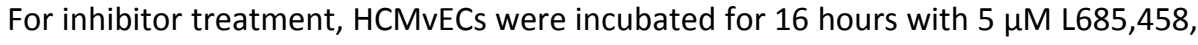
GI250423X, GW28026X or $20 \mu \mathrm{g} / \mathrm{mL}$ IMC-1121b or IMC-18F1 antibodies prior to stimulation with VEGF-A $A_{165}$. PAECs (Porcine Aortic Endothelial Cell), VEGFR-1/PAECs and VEGFR-2/PAECs ${ }^{22}$ were cultured in EGM-2MV medium without bFGF and VEGF$A_{165}$ (overnight) and, subsequently stimulated with $100 \mathrm{ng} / \mathrm{mL}$ VEGFA $A_{165}$ for 24 
hours. Cells were subcultured according to the manufacturer's protocol (Lonza) and viable cells count was determined using trypan blue staining and the Countess Automated cell Counter (Invitrogen).

\section{Plasmids and transfection}

Mouse Notch-ICD-1 and human NOTCH-ICD-4 were both cloned in pcDNA expression vectors. The pIRES2-EGFP (Clontech) containing the full-length human DLL4 coding region, DLL4-promoter constructs pGL3-hDLL4 6kb, the pGL3-hDLL4 (2616), the pGL3-hDLL4 Sacl (-1517) and the pGL3-hDLL4 Mscl (-931) were kindly provided by Prof. Dr Manfred Gessler and have been previously described in ${ }^{17}$. To obtain a minimal DLL4 promoter fragment lacking any putative RBP-Jk binding site the pGL3-hDLL4 Mscl (-931) was digested with Nrul and Pstl to remove the RBP-Jk binding site, blunted and religated. Constitutively active FOXC2-VP16 (caFOXC2) pCB6 expressing vector was kindly provided by Prof. Ormond A. MacDougald and has been previously described ${ }^{23}$. All transfection experiments were carried out in triplicate. HCMvECs were grown on $0.2 \%$ gelatin coated plates. Transfections were performed with lipofectamine LTX and PLUS reagent (Invitrogen). After 24 or 48 hours of culture RNA or proteins were isolated and analyzed by RT-qPCR or Western Blotting, respectively.

\section{Luciferase Reporter Assay}

HCMvECs seeded on $0.2 \%$ gelatin in PBS coated plates were transiently transfected with $0.6 \mu \mathrm{g}$ of either inducible RPB-Jk responsive firefly luciferase construct (SuperArray Bioscience Corporation, Frederick, MD, USA). After 24 hours stimulation with VEGF-A $A_{165}$ or inhibitors, the cells were harvested using Dual Glo luciferase assay system (Promega, Leiden, the Netherlands) and reporter activity was measured on a PE Victor3 plate reader (Perkin Elmer, Groningen, the Netherlands). PAECs were transiently transfected with pcDNA NICD-1, NICD-4 or vector alone along with the $6 \mathrm{~kb}$ DLL4- promoter firefly luciferase construct or with the deletional constructs of this promoter $(-2616,-1587,-931$ or $-931-D e l e t i o n(D)$, respectively). The renilla luciferase reporter plasmid was cotransfected as internal control for defining transfection efficiency. After 24 hours of transfection, cells were harvested and luciferase activity was assessed as reported above.

RNA isolation and RT-qPCR and Protein Extraction.

After each experiment RNA was isolated by using RNeasy micro-kit Qiagen (Qiagen, $\mathrm{GmbH}$, Hilden, Germany). A total of $100 \mathrm{ng}$ RNA per sample was subjected to reverse transcription (RT). QPCR was performed using Superscript ${ }^{\mathrm{TM}}$ III Platinum Two-step qRT-PCR kit with SYBR green (Invitrogen) and primer concentration of 10 $\mu \mathrm{M}$ according to Van den Akker et al ${ }^{24}$. PCR Primers used were. human $\beta$-ACTINsense: $\quad$ 5'-ATCCTCACCCTGAAGTACCC-3', $\beta$-ACTIN-antisense: $\quad$ 5'-CACGCAGCTC 
ATTGTAGAAG-3'; GADPH-sense: 5'-GCCTCAAGATCATCAGCAAT-3' GADPH-antisense: 5'-GGACTGTGGTCATGAGTCCT-3'; DLL4-sense: 5'-ACAACTTGTCGGACTTCCAG-3', DLL4-antisense: 5'-CAGCTCCTTCTTCTGGTTTG-3'; HES-1-sense: 5'CCAAAGACAGCATCTGAGCA-3', HES-1-antisense: 5'-GCCGCGAGCTATCTTTCTT-3'; porcine $\beta$-ACTIN -sense: 5'-gcatcctgaccctcaagtac-3, i $\beta$-ACTIN -antisense: 5'cacgcagctcgttgtagaag-3'; GADPH-sense: 5'-gtgtcggttgtggatctga-3', GADPHantisense 5'-cctgcttcaccaccttctt-3'; DLL-4-sense: 5'-tgtcgcaatggaggtagctgcaag-3', DLL-4-antisense 5'-aaggtgctatgttcgcagtgcagg-3'. Primers were designed with oligoperfect ${ }^{\mathrm{TM}}$ Designer (Invitrogen), Primer3 and Mfold (http: // www.idtdna.com/ scitools/ Application/ mfold/) and were synthesized by Eurogentec (Seraing, Belgium). qPCR reactions were run on a BioRad MiQ Real Time PCR detection System (BioRad, Veenendaal, the Netherlands). Proteins were isolated using RIPA lysis buffer supplemented with PMSF, protease inhibitor cocktail and sodium orthovanadate (Santa Cruz, Santa Cruz, USA). The protein concentration was measured using Micro BCA (Pierce, Rockford, USA). For Western Blot analysis, XT reducing agent and sample buffer (Biorad) were added to the total cell lysates, samples and were heated at $95^{\circ} \mathrm{C}$ for 5 minutes, loaded on a $4-12 \%$ gradient Criterion XT Bis-Tris gel and were, subsequently, subjected to electrophoresis in MOPS running buffer (BioRad) at $200 \mathrm{~V}$ for 55 minutes. Proteins were transferred to a PVDF membrane (BioRad) at $30 \mathrm{~V}$ overnight using Tris-glycin transfer buffer. Nonspecific binding of the antibodies was blocked by incubating with $3 \%$ milk powder in TBS supplemented with Tween-20 (TBS-T) for 1 hour. The membranes were exposed to anti-NOTCH1 (Santa Cruz sc-6014) or anti-NOTCH-4 (Santa Cruz sc-5594), or antiDLL4 (R\&D, MAB1389) antibodies and were, subsequently, incubated with biotinylated Goat-anti-Rabbit IgG (Vector Labs, Burlingame, USA BA-1000) or biotinylated Rabbit anti-Rat (Vector BA-9001) secondary antibody. The detection was performed by using SuperSignal West Femto or Pico substrate (Pierce) and was visualized with the ChemiDoc XRS System (BioRad).

Immunofluorescence Staining for DLL4 and eGFP.

HCMVECs (Lonza, Switzerland) were cultured on 4 wells slides (BD, Franklin Lakes, USA) until confluence and transfected with pIRES2-EGFP or pDLL4IRES2-EGFP. After 48 hours cell were incubated for 1 hour with the primary rabbit-anti human DLL4 antibody (Ab; R\&D; MAB1389). Slides were washed and incubated with $2 \%$ rabbit serum and secondary Ab Alexa-Fluo594 Rabbit anti-Rat (Invitrogen). Photos were taken using a Leica DFC350 FX digital camera.

\section{Statistical Analysis.}

Statistical significance was determined by applying one-way ANOVA/Tuckey (SigmaStat Software, San Jose, USA) testing. Values are expressed as mean \pm SEM of 3 experiments. Probability values of $<0.05$ were considered significant. 


\section{Results}

VEGFR-2 mediated DLL4 expression involves NOTCH signaling in endothelial cells.

To investigate the effect of VEGFR-1 and VEGFR-2 signaling on DLL4 expression, HCMvECs were exposed to receptor specific ligands, i.e. Placenta Growth Factor-2 (PIGF-2) for VEGFR-1, VEGF-E for VEGFR-2, and VEGF-A165 for both receptors ${ }^{25-27}$. Both VEGF-A165 and VEGF-E increased DLL4 expression, whereas PIGF-2 had no significant effect, suggesting a predominant involvement of VEGFR-2 (Fig. 1A). In accordance, specific inhibition of VEGFR-2 by the IMC-1121b antibody (Fig. 1B) prevented VEGF-A165 induced DLL4 expression, whereas VEGFR-1 inhibition with IMC 18F1 had no effect (Fig 1B). Final confirmation for the dominance of VEGFR-2 signaling, came from PAEC cell lines that overexpress either the human VEGFR-1 or VEGFR-2 ${ }^{22}$. In line with the HCMvEC results, VEGF-A165 induced DLL4 only in VEGFR2 transfected but not in VEGFR-1 transfected PAECs (Fig.1C).

VEGFA induced DLL4 expression in HCMvECs was abrogated by blockage of NOTCH signaling with $\gamma$-secretase- and ADAMs inhibitors (Fig. 1D), suggesting a role for $\mathrm{NOTCH}$ signaling. To further substantiate that VEGF-A165 activated NOTCHsignaling, RBP-JK luciferase activity and NOTCH target gene HES-1 expression were assessed. VEGF-A165 induced RPB-Jk promoter activity and HES-1 expression (Figs. $1 \mathrm{E} \& 1 \mathrm{~F}$ ), an effect that was prevented by NOTCH inhibition (Figs.1E \& 1F).

Activated NOTCH-signaling induces DLL4 expression by activating DLL4 promoter. $\mathrm{NOTCH}-1$ and NOTCH-4 are the main mediators of NOTCH-signaling in the endothelium $^{28,29}$. In order to define a direct effect of $\mathrm{NOTCH}$ signaling on DLL4 expression, HCMvECs were transfected with the intracellular domain of NOTCH-1 and -4 (NICD-1 and NICD-4, respectively) or empty vector. Both NICD-1 and -4 induced DLL4 mRNA and protein expression (Figs. 2A \& C lower panel). Endogenous NOTCH signaling was not required for this stimulation as NICD-1 and -4 overexpression had the same effect in the presence of $\gamma$-secretase inhibition (Fig. 2B).

The DLL4 promoter has previously been described to contain a forkhead binding element (FBE) and DLL4 expression was reported to be induced by VEGFA in a FOXC mediated manner ${ }^{15,16}$. Furthermore, the DLL4 promoter sequence also contains several RBP-Jk binding sites ${ }^{17}$, suggesting that NOTCH signaling might regulate DLL4 expression as well. 


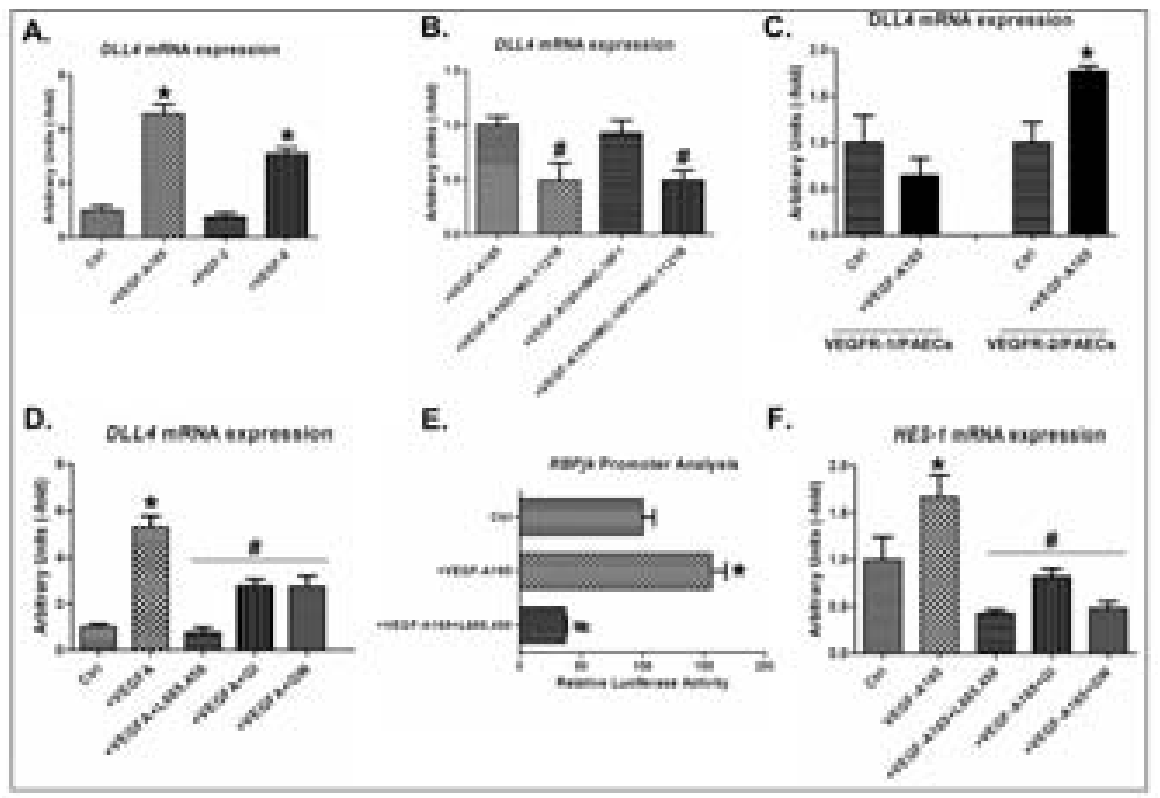

Figure 1 VEGF-A165-induced DLL4 expression is VEGFR-2 and NOTCH-signaling mediated.

A. DLL4 mRNA expression in HCMvECs treated with VEGF-A165, VEGF-E or PIGF-2 compared to the expression of non treated cells as control. B. DLL4 mRNA expression in HCMvECs treated with VEGFA165 or VEGF-A165 along with IMC-1121b (hVEGFR-2 inhibitor), or IMC-18F1 (hVEGFR-1 inhibitor) or IMC-1121b and IMC-18F1. C. DLL4 mRNA expression in VEGFR-2/PAECs (right) and VEGFR-1/PAECs (left) upon stimulation with or without VEGF-A165. D. DLL4 mRNA expression in HCMvECs stimulated with or without VEGF-A165 and VEGF-A165 along with L685,458 ( $\gamma$-secretase inhibitor), GI254023X (ADAM-10 inhibitor) or GW280264X (ADAM-10/ADAM-17 inhibitor). E. Luciferase activity of HCMvECs cell lysates transiently transfected with RPB-Jk -luciferase reporter construct and cultured with or without VEGF-A165 or VEGF-A165 along with L685,458 ( $\gamma$-secretase inhibitor). F. HES-1 mRNA expression in HCMvECs stimulated with or without VEGF-A165 and VEGF-A165 along with L685,458 ( $\gamma$-secretase inhibitor), GI254023X (ADAM-10 inhibitor) or GW280264X (ADAM-10/ADAM-17 inhibitor). Data represent mean $\pm S E(n=3)$. One way Anova test: ${ }^{*}=$ significantly different from control $p<0.05 ; \#=$ significantly different from VEGF-A165 $p<0.05$.

To discriminate between FOXC and NICD/RBP-Jk regulated DLL4 expression several deletional DLL4 promoter fragments were analyzed for their transactivation activity upon NICD-1 and -4 activation in PAECs. These promoter fragments lacked the FBE site and contained either three (-2616), two (-1587), one (-931) or no (-931-D) RBPJk binding site, respectively ${ }^{17}$. The $6 \mathrm{~kb}$ DLL4 promoter fragment was significantly stimulated when co-transfected with NICD-1 or NICD-4 (Fig. 3A). FBE deletion in the presence of all three RBP-Jk sites resulted in NICD-1 transactivation that was comparable to the full length promoter. Significant transactivation was also apparent after NICD-4 overexpression, although less effective than NICD-1 (Fig. 3A). 
The shorter -1587 and -931 constructs, containing two and one RBP-Jk binding sites, respectively, showed reduced but still significant induction by NICD-1. Although NICD-4 transactivated the -1587 fragment that contained two RBP-Jk binding sites, NICD-4 was unable to induce promoter activity in the -931 DLL4 promoter fragment that presented with only one RBP-Jk binding site. As expected, no NICD transactivation effect was observed for the -931-D fragment that lacked both the FBE and all RBP-Jk binding sites (Fig 3A).

Like in HCMvECs, NICD-1 and -4 overexpression (Fig. 3C) upregulated DLL4 mRNA expression (Fig. 3B) in PAECs. Transfection of both HCMvECs and PAECs with a constitutively active FOXC2 (caFOXC2) expressing vector did not alter DLL4 expression (Fig.3D), underlining that in endothelial cells the FOXC2 pathway seems redundant for NOTCH stimulated DLL4 expression.

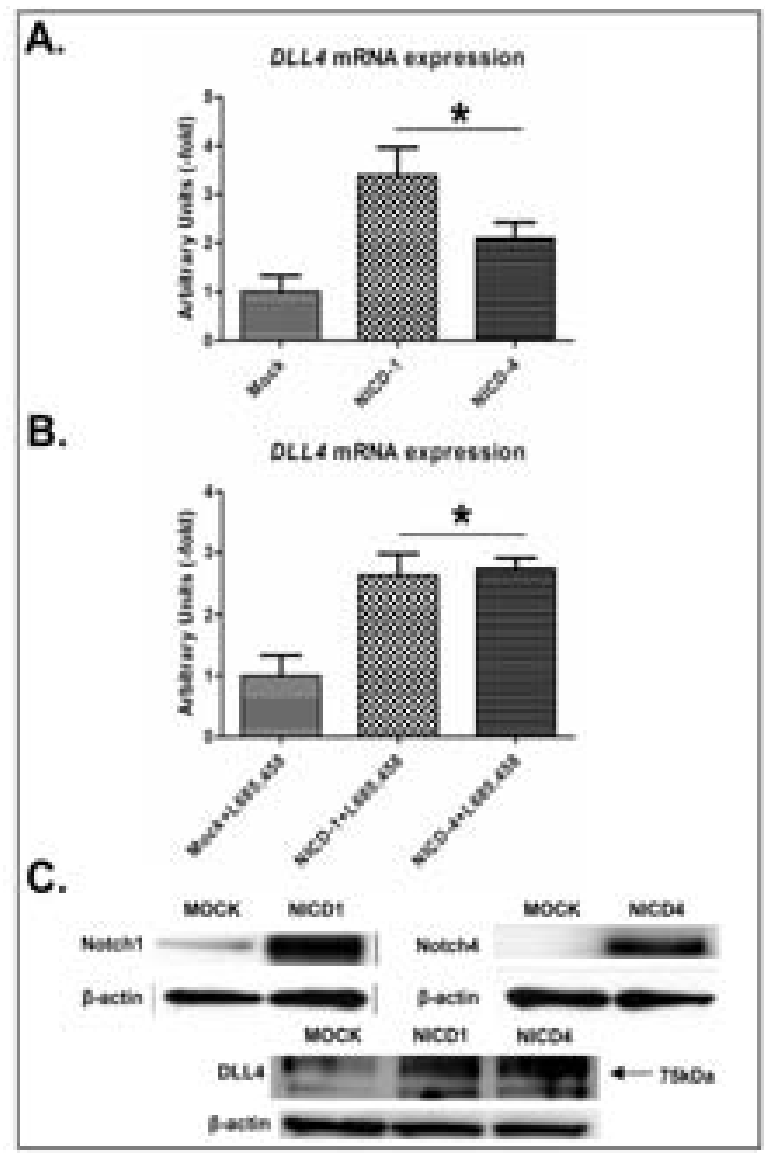

Figure 2 NICD-1 and NICD-4 induced DLL4 expression in HMvECs. A and B DLL4 MRNA expression in HCMvECs transiently transfected with NICD1, NICD-4 or pcDNA empty vector as control with or without L685,458. Data represent mean \pm SE $(n=3)$. One way Anova test: * = significantly different from control (mock) $\mathrm{p}<0.05$. C. Western Blot analysis of protein extracts from HCMvECs transfected with NICD-1 and NICD-4. Membranes were probed with anti-NOTCH-1(upper left panel), anti-NICD-4 (upper right panel) or anti-DLL4 (lower panel). 


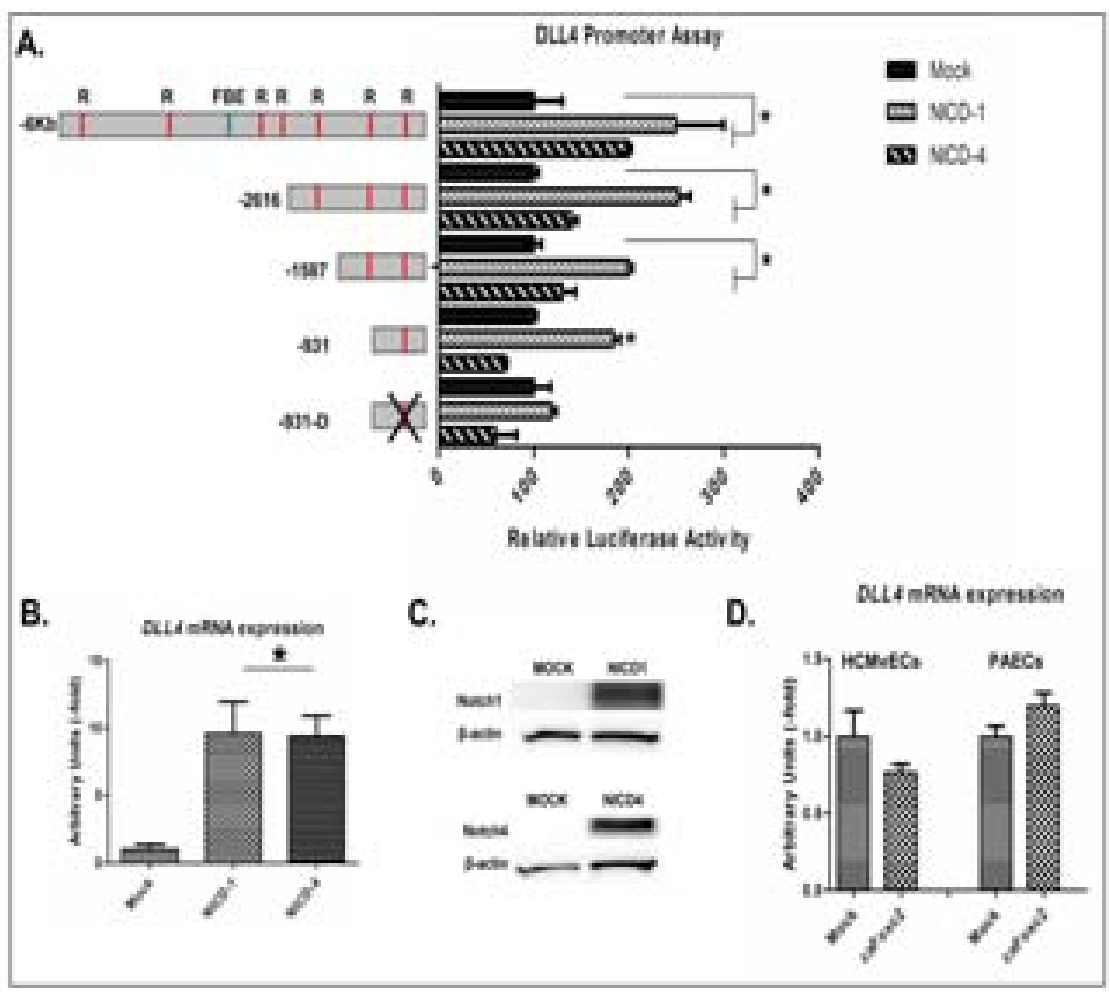

Figure 3 Induction of DLL4 promoter activity by NICD-1 and NICD-4.

A. Luciferase assay activity from PAE cells contransfected with a $6 \mathrm{~kb}$ DLL4 promoter luciferase reporter or shortened fragments of the $6 \mathrm{~kb}$ promoter containing three (-2616), two (-1587), one (-931) or none (-931-D) RPB-Jk binding site along with NICD-1, NICD-4 or empty pcDNA (mock) vector. Schematic drawing of the $6 \mathrm{~kb}$ DLL4 promoter and of the shortened fragments -2616, -1587, -931 and -931-D (relative to the transcriptional initiation site) with potential RPB-Jk (R) sites indicated by red bars, FBE (Forkhead binding site) indicated by a green bar and deletion of (R) indicated by the black cross. $B$. DLL4 mRNA expression in PAECs transiently transfected with NICD-1 and NICD-4 and pcDNA as control. Data represent mean \pm SE $(n=3)$. One way Anova test: * = significantly different from control (mock), $p<0.05$. C. Western Blot analysis of protein extracts from PAECs transfected with NICD-1 (upper panel) and NICD-4. (lower panel). Results are from one representative experiment. D. DLL4 mRNA expression in HCMvECs (left panel) and PAECs (right panel) transiently transfected with constitutively active FOXC2 (caFOXC2) and empty vector as control.

\section{DLL4-induced NOTCH-signaling upregulates DLL4 expression}

To address a potential DLL4-driven positive feed-forward loop, rDLL4 protein was used to stimulate the NOTCH pathway in HCMvECs. Similar to VEGFA, rDLL4 was able to increase the expression of DLL4. NOTCH signaling blockage by $\gamma$-secretase and ADAMs inhibitors prevented rDLL4-induced DLL-4 expression (Fig. 4A). The activation of NOTCH signaling by rDLL4 was further assessed by RBP-Jk luciferase promoter analysis. rDLL4 stimulation induced transactivation of RBP-Jk promoter, that was prevented by NOTCH inhibitors (Fig. 4B). To investigate whether rDLL4- 
induced DLL4 expression depends on VEGFR-2 signaling we cultured HMCVECs on rDLL4 along with specific VEGFR-2 or/and VEGFR-1 inhibitors and analyzed DLL4 expression. The inhibition of VEGFR-2, of VEGFR-1 or of both receptors did not affect rDLL4-induced DLL4 expression suggesting that VEGF signaling is not required for the positive feed-forward circuit (Fig. 4C).

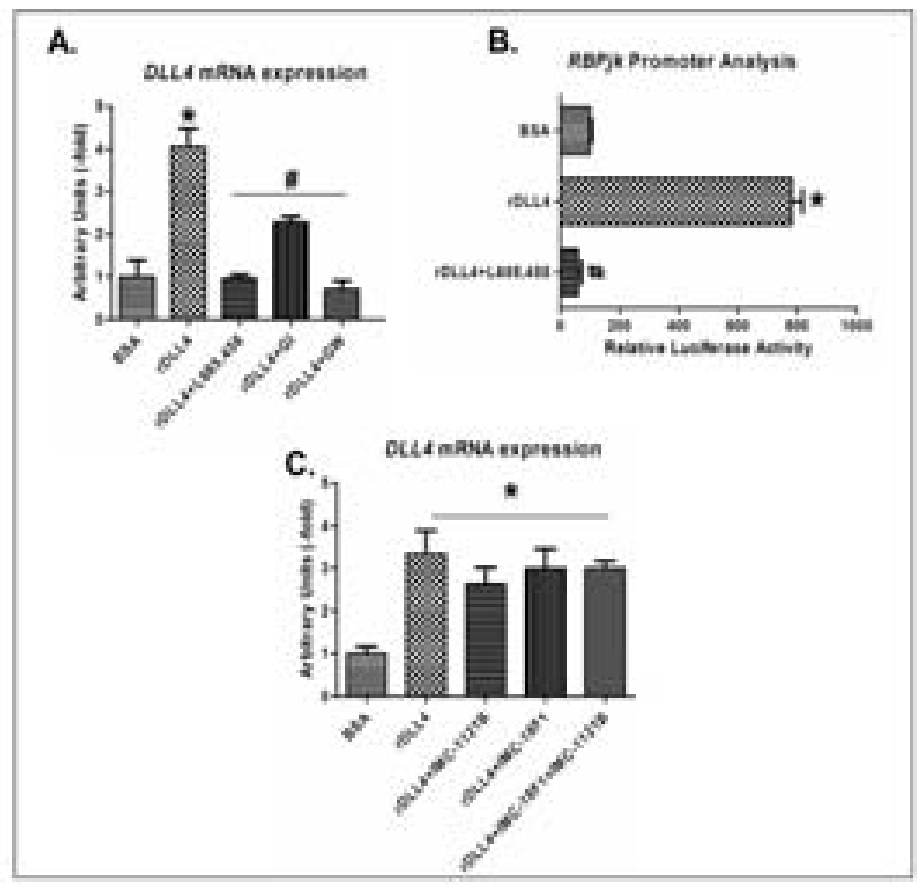

Figure 4 rDLL4 activated NOTCH-signaling induced DLL4 expression.

A. DLL4 mRNA expression in HCMvECs stimulated with BSA as control, rDLL4 or rDLL4 along with L685,458 ( $\gamma$-secretase inhibitor), or GI254023X (ADAM-10 inhibitors) or GW280264X (ADAM-10 and ADAM-17). B. Luciferase activity of HCMvECs cell lysates transiently transfected with RPB-Jk-luciferase reporter construct and stimulated with BSA as control, rDLL4 protein or with rDLL4 and L685,458. C. DLL4 mRNA expression in HCMvECs stimulated with BSA as control, rDLL4 or rDLL4 along with IMC1121b (hVEGFR-2 inhibitor), or IMC-18F1 (hVEGFR-1 inhibitor) or IMC-1121b and IMC-18F1. Data represent mean $\pm S E(n=3)$. One way Anova test: * = significantly different from control $(B S A) p<0.05 ; \#$ = significantly different from rDLL4.

\section{DLL4 induces propagation of endogenous DLL4 expression in adjacent cells}

As NOTCH is an inter-cellular signaling pathway, we hypothesized that $\mathrm{NOTCH}$ induced DLL4 expression taking place in one specific cell would eventually induce NOTCH signaling and, subsequently DLL4 expression in its neighboring cell. To test this hypothesis, HCMvECs were transfected with pIRES2-eGFP or pDLL4IRES2-eGFP with or without NOTCH signaling inhibitor $(L 685,458)$ and DLL4 expression was analyzed by immunofluorescence staining. eGFP (green) signal allows to 
discriminate between transfected and non-transfected cells (Figs. 5A-C GFP). pIRES2-eGFP transfected control cells presented relatively weak DLL4 expression (red staining) in their surrounding cells (Fig. 5A, DLL4). In contrast, the pDLL4IRES2eGFP transfected cells revealed high DLL4 level and prominent DLL4 staining was also detected in the non-transfected surrounding cells (Fig. 5B, DLL4). The pDLL4IRES2-eGFP expressing cells treated with L685,458 had high DLL4 expression, however no DLL4 was detected in the surrounding non-transfected cells (Fig. 5C, DLL4) indicating that inhibition of NOTCH signaling was sufficient to prevent the DLL4-induced positive feed-forward loop.

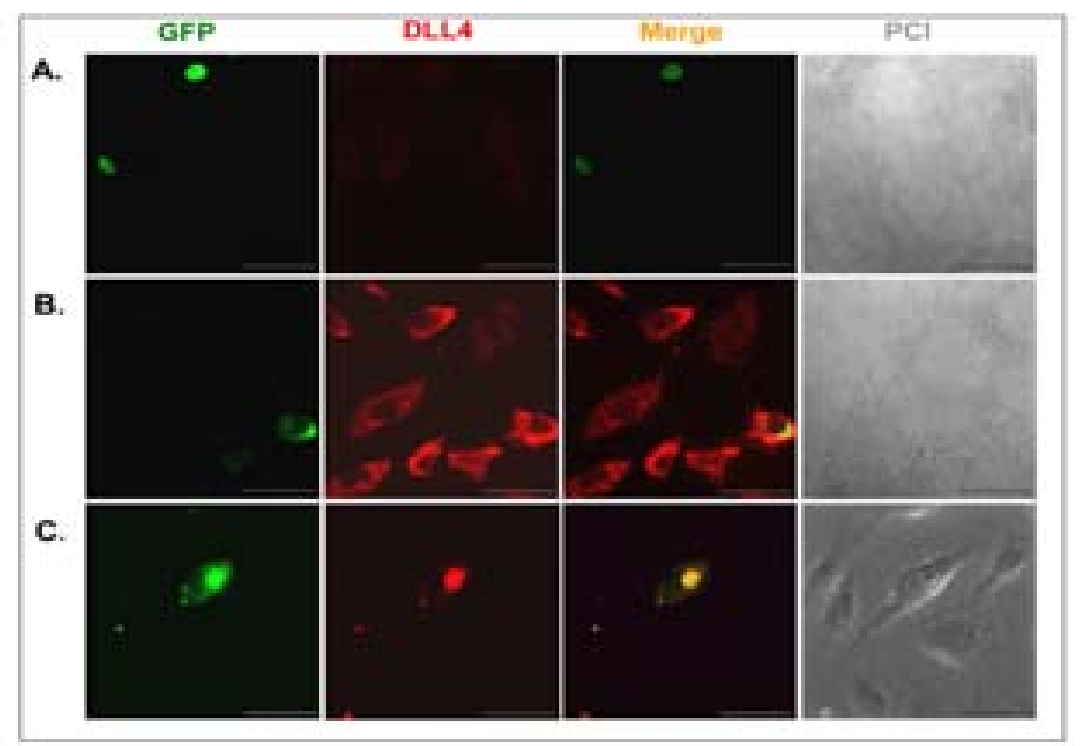

Figure 5 DLL4 overexpressing cells induce endogenous DIl4 expression in the neighboring and adjacent cell. DLL4 immuno-fluorescent labeling pIRES-eGFP transfected HCMvECs versus pIRESDLL4-eGFP transfected HCMvECs with or without L685,458. eGFP (green) signal allows to discriminate between transfected and non-transfected cells (Fig. 5 A, B and C; GFP). pIRES-DLL4-eGFP transfected ECs revealed higher DLL4 staining (red) (B and C; DLL4) compared to pIRES-eGFP transfected cells ( $A$; DLL4). Cells surrounding pIRES-eGFP-DLL4 transfected cells also showed increased DLL4 expression (B; DLL4) compare to cells surrounding pIRES- eGFP transfected cells (A; DLL4). This effect was prevented by adding the NOTCH-signaling inhibitor L685,458 (C, DLL4). Merged images between eGFP and DLL4 staining (Fig. 5, A,B,C; Merge) and PCl (Phase Contrast microscopy) images (Fig. 5, A,B,C; $\mathrm{PCl})$ are also reported. Scale bars: $50 \mu \mathrm{m}$. 


\section{Discussion}

The VEGF and NOTCH signaling pathways and their intricate interactions are indispensable for regulating blood vessel formation. VEGF-A has been reported to modulate the expression of NOTCH members in arterial endothelial cells. VEGFR-2 acts as the main receptor in mediating VEGF signaling in vascular endothelial cells ${ }^{22,}$ 30,31 and likewise has been suggested to positively regulate NOTCH/DELTA expression $^{10,11,21,24,32}$. Also a reciprocal effect of $\mathrm{NOTCH}$ signaling on the expression of VEGFR-2 has been reported, i.e. a reduction in VEGFR-2 and an increase in VEGFR-1 expression with subsequent drop in VEGFA responsiveness ${ }^{32}$.

Accordingly, we show that in primary adult HCMvECs, the expression of DLL4 is stimulated by the VEGFR-2 acting ligands VEGF-A165 and VEGF-E, and not by PIGF-2. Intriguingly, no other NOTCH ligands beside DLL4 were affected by VEGF-A-related endothelial signaling (data not shown) suggesting a selectivity of VEGF-A-related signaling in primary adult microvascular ECs. The essential role of VEGFR-2 was confirmed in porcine aortic endothelial cells expressing only one of the receptors and with a specific VEGFR-2 inhibitor. The presented data clearly show that upregulation of DLL4 by VEGF-A is NOTCH signaling dependent. VEGF-A failed to induce DLL4 expression when NOTCH signaling was inhibited, indicating the requirement of activated NOTCH for this process. In addition, NOTCH signaling alone was sufficient to induce DLL4 expression as shown by NICD-1 and NICD-4 overexpression. A comparable effect of VEGF-A induced DLL4 expression was described for embryonic ${ }^{33}$ and human bone-marrow derived mesenchymal stem cells ${ }^{4}$.

We show that both NICD-1 and NICD-4 were able to transactivate the DLL4 promoter. Previous reports ${ }^{15,16}$ indicated that the FOXC2 transcription factor can serve as a mediator for VEGF-A induced DLL4 expression by interacting with a FBE located in the DLL4 promoter. Besides the FBE, multiple RBP-Jk binding sites are present in the DLL4 promoter sequence, providing the potential molecular cues for NOTCH induced expression. Indeed, NICD-1 effectively transactivated both the full length DLL4 promoter and FBE deleted DLL4 promoter sequences providing evidence that the FOX transcription factor pathway does not mediate VEGF-induced $\mathrm{NOTCH}$ activation in the endothelium. Accordingly, overexpression of a constitutively active FOXC2 transcript did not increase DLL4 expression. The FBE lacking $-2616,-1587$ and -931 promoter constructs, showed a transactivation profile that suggests dependency on the number of RBP-Jk binding sites. As expected, both NICD-1 and -4 were unable to stimulate the -931- DLL4 promoter fragment that lacked any RBP-Jk binding site. NICD-4 had a similar profile of transactivation as NICD-1 with respect to -2616 and -1587 fragments but, in accordance with previous studies, at lower levels ${ }^{34}$. This difference likely relates to the presence of a Cterminal autonomous transactivation domain (TAD) in the NICD-1, which acts independently of RBP-Jk. Such TAD sequence is absent in the NICD-4 and 
consequently, it requires RBP-Jk to initiate transcription ${ }^{35}$. Therefore, the reduced induction capacity of NICD-4 is likely related to the number of RBP-Jk that are present in the shortened -2616 and -1587 DLL4 promoter fragments. Accordingly, no effect was shown by NICD-4 for the -931 DLL4 promoter fragment, that contained only one RBP-Jk binding site, whereas NICD-1 could still effectively transactivate this promoter.

NOTCH is typically activated by one its ligands, including membrane bound DLL4. Similar to VEGF-A therefore, NOTCH activation by surface bound recombinant DLL4 induced DLL4 expression in HCMvECs. Inhibition of NOTCH signaling, but not of VEGF signaling, was sufficient to prevent this induction. These data indicate that activated NOTCH can induce DLL4 expression and transactivate the DLL4 promoter in absence of FOXC transcription factors and support the observation that NOTCH can induce DLL4 expression independently of FOXC2-mediated VEGF signaling. To our knowledge, these results show for the first time that DLL4 induces its own expression in endothelial cells, most likely through direct NICD mediated transcriptional regulation. VEGF-signaling also induced the upregulation of HES-1 and RBP-Jk transactivation, indicating that VEGF-A activates NOTCH signaling. This activation is likely not secondary to ligand overexpression, as NOTCH inhibition prevented the VEGF-A-induced expression of DLL4.

The link between VEGF-A and NOTCH signaling most likely relates to VEGFR-2 increased ADAM expression and activity. In a previous study we showed that ADAM-expression and protease activity (i.e. sheddase) in HUVECs and VEGFR2/PAEC was increased upon VEGF-A $A_{165}$ stimulation $^{36}$. VEGF-A has also been reported to induce the expression of $A D A M-10$ and to enhance shedding of VEGFR-2 and its co-receptor NRP1 by activating ADAM-10 and ADAM-17, respectively ${ }^{14}$. Although this mechanism has been postulated to modulate VEGF-A signaling ${ }^{14}$, VEGF-A increased ADAM sheddase activity might potentiate NOTCH signaling as well. In line with our observations Hainaud et al $^{37}$ showed an accumulation of cleaved NOTCH-4 in VEGF-A-treated HUVECs with concomitant upregulation of the ADAM and Presinilin (part of the $\gamma$-secretase complex) expression.

The involvement of ADAM-10 and ADAM-17 in NOTCH processing and signaling has been unequivocally established ${ }^{38-42}$. ADAM-10 mutant mice phenotypically resemble Notch-1 null mutants and die at embryonic day 9.5, whereas ADAM-17 mutants are viable ${ }^{43}$. Among others Bozkulak and Weinmaster ${ }^{41}$ showed that NOTCH1 is a substrate for both ADAM-10 and ADAM-17 and, although ADAM-10 was essentially required for ligand induced NOTCH-1 signaling, ADAM-17 mediated ligand independent signaling. The importance of ADAM in NOTCH signaling was also reflected by our experiments showing that inhibition of ADAM activity abrogated the VEGF-A induced expression of DLL4 and the NOTCH transcription factor HES-1. In addition, rDLL4 induced DLL4 expression was depressed by blockage of ADAM 
activity as well. Altogether these data indicate that VEGF-A activates ADAM, which is required for NOTCH signaling activation.

The ability of the activated NOTCH receptor to specifically induce the expression of its ligand would provide endothelial cells a mechanism by which NOTCH signaling is distributed and propagated between communicating cells with initially a limited amount of ligand. A comparable positive feed-forward mechanism has recently been described to regulate the interaction between endothelial and mural cells. JAGGED-1 ligand expressing endothelial cells induced NOTCH-3 signaling activation in mural cells ${ }^{44}$, that in turn promote JAGGED-1's own expression. In this way mural cells can interact more efficiently with endothelial cells and can activate NOTCH signaling of neighboring cells, maintaining and distributing the signal to other mural cells. In the setting of membrane bound ligands the positive feed-forward mechanism is especially attractive to expand the signal to distant cells. The regulation of ligand expression by NOTCH receptors has already been described in Drosophila $^{45}$. NOTCH signaling was found to increase the expression of Delta and Serrate through a positive feedback loop during the regulation of the dorsoventral boundary of the developing wing $^{45}$. Likewise, in vertebrates, Delta-like ligands are required during somitogenesis for the maintenance of somite borders and accumulate in a Notch-dependent manner ${ }^{46-48}$.

The expression pattern of DLL4 ligand in arteries and sprouting vessels affects spatial distribution of NOTCH signaling in endothelial cells. During sprouting, tip endothelial cells will express high DLL4 levels and low NOTCH activity, whereas their neighbor stalk cells will have high NOTCH activity but low DLL4 expression. However, a static situation in which a subpopulation of cells presents high level of DLL4 and low NOTCH signaling is unlikely. NOTCH signaling is in fact required for arterial specification and lack or reduction of NOTCH signaling would cause loss of arterial identity. Here we show, that in contrast to eGFP-transfected cells, DLL4eGFP ECs were able to induce DLL4 expression of non-transfected surrounding ECs, through a feed-forward mechanism, that was NOTCH signaling dependent. Coordinated activation of $\mathrm{NOTCH}$ signaling would produce a wave of DLL4 expression that provides periodic NOTCH signaling activation in each cell ${ }^{9}$. Such mechanism could be important to maintain an arterial phenotype along a blood vessel and for regulating spatial patterning of branching as well ${ }^{9,49}$.

In summary, stimulation of VEGFR-2 by VEGF-A165 up-regulates DLL4 expression by activating $\mathrm{NOTCH}$ signaling in HCMvECs, independently of FOXC2. The NOTCH involvement in regulating DLL4 expression indicates the existence of a feed-forward mechanism by which NOTCH signaling can be propagated between adjacent endothelial cells. 


\section{Aknowledgments}

We thank Dr. Bronek Pytowski from ImClone Systems Corporations for kindly providing us with the inhibitors IMC-18F1 and IMC-1121b and Dr. David Becherer from GlaxoSmithKline for the inhibitors GI254023X and GW280264X. We are grateful to Prof. Dr Manfred Gessler for providing us with the pGL3-hDll4 6kb, the pGL3-hDll4 (-2616) and the pGL3-hDll4 Sacl (-1587), pGL3-hDLL4 Mscl (-931) and pDLL4-IRES-eGFP constructs and to Prof. Ormond A. MacDougald for the FOXC2VP16-pCB6 construct and Martin Köhler for technical support. This research was supported by The Netherlands Heart Foundation (grant 2005B254 to M.P. and Dr. E. Dekker grant $200 T 034$ to M.D.) and the Marie Curie FP6 early stage researcher training (MEST-CT-2005-020706 to CARIM). 


\section{References}

1. Iso, T., Kedes, L. and Hamamori, Y., HES and HERP families: multiple effectors of the Notch signaling pathway, J Cell Physiol, 2003, 194: 237-255.

2. Gale, N. W., Dominguez, M. G., Noguera, I., Pan, L., Hughes, V., Valenzuela, D. M., Murphy, A. J., Adams, N. C., Lin, H. C., Holash, J., Thurston, G. and Yancopoulos, G. D., Haploinsufficiency of deltalike 4 ligand results in embryonic lethality due to major defects in arterial and vascular development, Proc Natl Acad Sci U S A, 2004, 101: 15949-15954.

3. Iso, T., Maeno, T., Oike, Y., Yamazaki, M., Doi, H., Arai, M. and Kurabayashi, M., DII4-selective Notch signaling induces ephrinB2 gene expression in endothelial cells, Biochem Biophys Res Commun, 2006, 341: 708-714.

4. Zhang, G., Zhou, J., Fan, Q., Zheng, Z., Zhang, F., Liu, X. and Hu, S., Arterial-venous endothelial cell fate is related to vascular endothelial growth factor and Notch status during human bone mesenchymal stem cell differentiation, FEBS Lett, 2008, 582: 2957-2964.

5. Krebs, L. T., Shutter, J. R., Tanigaki, K., Honjo, T., Stark, K. L. and Gridley, T., Haploinsufficient lethality and formation of arteriovenous malformations in Notch pathway mutants, Genes Dev, 2004, 18: 2469-2473.

6. Kim, Y. H., Hu, H., Guevara-Gallardo, S., Lam, M. T., Fong, S. Y. and Wang, R. A., Artery and vein size is balanced by Notch and ephrin B2/EphB4 during angiogenesis, Development, 2008, 135: 3755-3764.

7. Aranguren, X. L., Luttun, A., Clavel, C., Moreno, C., Abizanda, G., Barajas, M. A., Pelacho, B., Uriz, M., Arana, M., Echavarri, A., Soriano, M., Andreu, E. J., Merino, J., Garcia-Verdugo, J. M., Verfaillie, C. M. and Prosper, F., In vitro and in vivo arterial differentiation of human multipotent adult progenitor cells, Blood, 2007, 109: 2634-2642.

8. Trindade, A., Kumar, S. R., Scehnet, J. S., Lopes-da-Costa, L., Becker, J., Jiang, W., Liu, R., Gill, P. S. and Duarte, A., Overexpression of delta-like 4 induces arterialization and attenuates vessel formation in developing mouse embryos, Blood, 2008, 112: 1720-1729.

9. Phng, L. K. and Gerhardt, H., Angiogenesis: a team effort coordinated by notch, Dev Cell, 2009, 16: 196-208.

10. Lobov, I. B., Renard, R. A., Papadopoulos, N., Gale, N. W., Thurston, G., Yancopoulos, G. D. and Wiegand, S. J., Delta-like ligand 4 (DII4) is induced by VEGF as a negative regulator of angiogenic sprouting, Proc Natl Acad Sci U S A, 2007, 104: 3219-3224.

11. Liu, Z. J., Shirakawa, T., Li, Y., Soma, A., Oka, M., Dotto, G. P., Fairman, R. M., Velazquez, O. C. and Herlyn, M., Regulation of Notch1 and Dll4 by vascular endothelial growth factor in arterial endothelial cells: implications for modulating arteriogenesis and angiogenesis, Mol Cell Biol, 2003, 23: 14-25.

12. Segarra, M., Williams, C. K., Sierra Mde, L., Bernardo, M., McCormick, P. J., Maric, D., Regino, C., Choyke, P. and Tosato, G., DIl4 activation of Notch signaling reduces tumor vascularity and inhibits tumor growth, Blood, 2008, 112: 1904-1911.

13. Benedito, R., Trindade, A., Hirashima, M., Henrique, D., da Costa, L. L., Rossant, J., Gill, P. S. and Duarte, A., Loss of Notch signalling induced by Dll4 causes arterial calibre reduction by increasing endothelial cell response to angiogenic stimuli, BMC Dev Biol, 2008, 8: 117.

14. Swendeman, S., Mendelson, K., Weskamp, G., Horiuchi, K., Deutsch, U., Scherle, P., Hooper, A., Rafii, S. and Blobel, C. P., VEGF-A stimulates ADAM17-dependent shedding of VEGFR2 and crosstalk between VEGFR2 and ERK signaling, Circ Res, 2008, 103: 916-918.

15. Hayashi, H. and Kume, T., Foxc transcription factors directly regulate Dll4 and Hey2 expression by interacting with the VEGF-Notch signaling pathways in endothelial cells, PLoS One, 2008, 3: e2401.

16. Seo, S., Fujita, H., Nakano, A., Kang, M., Duarte, A. and Kume, T., The forkhead transcription factors, Foxc1 and Foxc2, are required for arterial specification and lymphatic sprouting during vascular development, Dev Biol, 2006, 294: 458-470.

17. Diez, H., Fischer, A., Winkler, A., Hu, C. J., Hatzopoulos, A. K., Breier, G. and Gessler, M., Hypoxiamediated activation of Dll4-Notch-Hey2 signaling in endothelial progenitor cells and adoption of arterial cell fate, Exp Cell Res, 2007, 313: 1-9. 
18. Wu, Y., Zhong, Z., Huber, J., Bassi, R., Finnerty, B., Corcoran, E., Li, H., Navarro, E., Balderes, P., Jimenez, X., Koo, H., Mangalampalli, V. R., Ludwig, D. L., Tonra, J. R. and Hicklin, D. J., Anti-vascular endothelial growth factor receptor-1 antagonist antibody as a therapeutic agent for cancer, Clin Cancer Res, 2006, 12: 6573-6584.

19. Miao, H. Q., Hu, K., Jimenez, X., Navarro, E., Zhang, H., Lu, D., Ludwig, D. L., Balderes, P. and Zhu, Z., Potent neutralization of VEGF biological activities with a fully human antibody Fab fragment directed against VEGF receptor 2, Biochem Biophys Res Commun, 2006, 345: 438-445.

20. Melenhorst, W. B., Mulder, G. M., Xi, Q., Hoenderop, J. G., Kimura, K., Eguchi, S. and van Goor, H., Epidermal growth factor receptor signaling in the kidney: key roles in physiology and disease, Hypertension, 2008, 52: 987-993.

21. Williams, C. K., Li, J. L., Murga, M., Harris, A. L. and Tosato, G., Up-regulation of the Notch ligand Delta-like 4 inhibits VEGF-induced endothelial cell function, Blood, 2006, 107: 931-939.

22. Waltenberger, J., Claesson-Welsh, L., Siegbahn, A., Shibuya, M. and Heldin, C. H., Different signal transduction properties of KDR and Flt1, two receptors for vascular endothelial growth factor, $J$ Biol Chem, 1994, 269: 26988-26995.

23. Gerin, I., Bommer, G. T., Lidell, M. E., Cederberg, A., Enerback, S. and Macdougald, O. A., On the role of FOX transcription factors in adipocyte differentiation and insulin-stimulated glucose uptake, $J$ Biol Chem, 2009, 284: 10755-10763.

24. van den Akker, N. M., Caolo, V., Wisse, L. J., Peters, P. P., Poelmann, R. E., Carmeliet, P., Molin, D. G. and Gittenberger-de Groot, A. C., Developmental coronary maturation is disturbed by aberrant cardiac vascular endothelial growth factor expression and Notch signalling, Cardiovasc Res, 2008, 78: 366-375.

25. Ferrara, N., Gerber, H. P. and LeCouter, J., The biology of VEGF and its receptors, Nat Med, 2003, 9: 669-676.

26. Meyer, M., Clauss, M., Lepple-Wienhues, A., Waltenberger, J., Augustin, H. G., Ziche, M., Lanz, C., Buttner, M., Rziha, H. J. and Dehio, C., A novel vascular endothelial growth factor encoded by Orf virus, VEGF-E, mediates angiogenesis via signalling through VEGFR-2 (KDR) but not VEGFR-1 (FIt-1) receptor tyrosine kinases, Embo J, 1999, 18: 363-374.

27. Olsson, A. K., Dimberg, A., Kreuger, J. and Claesson-Welsh, L., VEGF receptor signalling - in control of vascular function, Nat Rev Mol Cell Biol, 2006, 7: 359-371.

28. Shawber, C. J., Das, I., Francisco, E. and Kitajewski, J., Notch signaling in primary endothelial cells, Ann N Y Acad Sci, 2003, 995: 162-170.

29. Iso, T., Hamamori, Y. and Kedes, L., Notch signaling in vascular development, Arterioscler Thromb Vasc Biol, 2003, 23: 543-553.

30. Hiratsuka, S., Minowa, O., Kuno, J., Noda, T. and Shibuya, M., Flt-1 lacking the tyrosine kinase domain is sufficient for normal development and angiogenesis in mice, Proc Natl Acad Sci U S A, 1998, 95: 9349-9354.

31. Shalaby, F., Rossant, J., Yamaguchi, T. P., Gertsenstein, M., Wu, X. F., Breitman, M. L. and Schuh, A. C., Failure of blood-island formation and vasculogenesis in Flk-1-deficient mice, Nature, 1995, 376: 62-66.

32. Harrington, L. S., Sainson, R. C., Williams, C. K., Taylor, J. M., Shi, W., Li, J. L. and Harris, A. L., Regulation of multiple angiogenic pathways by Dll4 and Notch in human umbilical vein endothelial cells, Microvasc Res, 2008, 75: 144-154.

33. Lanner, F., Sohl, M. and Farnebo, F., Functional arterial and venous fate is determined by graded VEGF signaling and notch status during embryonic stem cell differentiation, Arterioscler Thromb Vasc Biol, 2007, 27: 487-493.

34. Kurooka, H., Kuroda, K. and Honjo, T., Roles of the ankyrin repeats and C-terminal region of the mouse notch1 intracellular region, Nucleic Acids Res, 1998, 26: 5448-5455.

35. Kato, H., Sakai, T., Tamura, K., Minoguchi, S., Shirayoshi, Y., Hamada, Y., Tsujimoto, Y. and Honjo, T., Functional conservation of mouse Notch receptor family members, FEBS Lett, 1996, 395: 221-224. 
36. Donners, M. M., Wolfs, I. M., Olieslagers, S., Mohammadi-Motahhari, Z., Tchaikovski, V., Heeneman, S., van Buul, J. D., Caolo, V., Molin, D. G., Post, M. J. and Waltenberger, J., A Disintegrin and Metalloprotease $10 \mathrm{Is}$ a Novel Mediator of Vascular Endothelial Growth Factor-Induced Endothelial Cell Function in Angiogenesis and Is Associated With Atherosclerosis, Arterioscler Thromb Vasc Biol.

37. Hainaud, P., Contreres, J. O., Villemain, A., Liu, L. X., Plouet, J., Tobelem, G. and Dupuy, E., The role of the vascular endothelial growth factor-Delta-like 4 ligand/Notch4-ephrin B2 cascade in tumor vessel remodeling and endothelial cell functions, Cancer Res, 2006, 66: 8501-8510.

38. Brou, C., Logeat, F., Gupta, N., Bessia, C., LeBail, O., Doedens, J. R., Cumano, A., Roux, P., Black, R. A. and Israel, A., A novel proteolytic cleavage involved in Notch signaling: the role of the disintegrinmetalloprotease TACE, Mol Cell, 2000, 5: 207-216.

39. Delwig, A. and Rand, M. D., Kuz and TACE can activate Notch independent of ligand, Cell Mol Life Sci, 2008, 65: 2232-2243.

40. Tian, L., Wu, X., Chi, C., Han, M., Xu, T. and Zhuang, Y., ADAM10 is essential for proteolytic activation of Notch during thymocyte development, Int Immunol, 2008, 20: 1181-1187.

41. Bozkulak, E. C. and Weinmaster, G., Selective use of ADAM10 and ADAM17 in activation of Notch1 signaling, Mol Cell Biol, 2009, 29: 5679-5695.

42. van Tetering, G., van Diest, P., Verlaan, I., van der Wall, E., Kopan, R. and Vooijs, M., Metalloprotease ADAM10 is required for Notch1 site 2 cleavage, J Biol Chem, 2009, 284: 31018-31027.

43. Hartmann, D., de Strooper, B., Serneels, L., Craessaerts, K., Herreman, A., Annaert, W., Umans, L., Lubke, T., Lena Illert, A., von Figura, K. and Saftig, P., The disintegrin/metalloprotease ADAM 10 is essential for Notch signalling but not for alpha-secretase activity in fibroblasts, Hum Mol Genet, 2002, 11: 2615-2624.

44. Liu, H., Kennard, S. and Lilly, B., NOTCH3 expression is induced in mural cells through an autoregulatory loop that requires endothelial-expressed JAGGED1, Circ Res, 2009, 104: 466-475.

45. de Celis, J. F. and Bray, S., Feed-back mechanisms affecting Notch activation at the dorsoventral boundary in the Drosophila wing, Development, 1997, 124: 3241-3251.

46. Bettenhausen, B., Hrabe de Angelis, M., Simon, D., Guenet, J. L. and Gossler, A., Transient and restricted expression during mouse embryogenesis of DIl1, a murine gene closely related to Drosophila Delta, Development, 1995, 121: 2407-2418.

47. Hrabe de Angelis, M., McIntyre, J., 2nd and Gossler, A., Maintenance of somite borders in mice requires the Delta homologue DII1, Nature, 1997, 386: 717-721.

48. Jen, W. C., Wettstein, D., Turner, D., Chitnis, A. and Kintner, C., The Notch ligand, X-Delta-2, mediates segmentation of the paraxial mesoderm in Xenopus embryos, Development, 1997, 124: 1169-1178.

49. Gerhardt, H., VEGF and endothelial guidance in angiogenic sprouting, Organogenesis, 2008, 4: 241246. 


\section{Chapter 3}

\section{ADAM-10 is a novel mediator of VEGF- induced endothelial cell function in angiogenesis and associated with atherosclerosis}

Marjo M.P.C. Donners ${ }^{1}$, Ine M.J. Wolfs1, Servé Olieslagers ${ }^{1}$, Zeynab MohammadiMotahhari ${ }^{2}$, Vadim Tchaikovski ${ }^{1}$, Sylvia Heeneman ${ }^{3}$, Jaap D. van Buul ${ }^{4}$, Vincenza Caolo $^{5}$, Daniël G.M. Molin ${ }^{5}$, Mark J. Post ${ }^{5}$, and Johannes Waltenberger ${ }^{1,2}$.

Departments of ${ }^{1}$ Cardiology, ${ }^{3}$ Pathology and ${ }^{5}$ Physiology, Cardiovascular Research Institute Maastricht, Maastricht University, Maastricht, the Netherlands; ${ }^{2}$ Department of Internal Medicine II, UIm University Medical Center, Ulm, Germany; ${ }^{4}$ Department of Molecular Cell Biology, Sanquin Research and Landsteiner Laboratory, Academic Medical Center, University of Amsterdam, Amsterdam, the Netherlands.

Modified after Arterioscler. Thromb. Vasc. Biol. 2010; 30:2188-95. 



\begin{abstract}
Neovascularization has been associated with atherosclerotic plaque growth, plaque instability, and elevated risk of clinical complications. VEGF is a key angiogenesis factor associated with plaque angiogenesis. Using a Yeast-two-hybrid assay we identified $\underline{A}$ Disintegrin $\underline{\text { And }}$ Metalloprotease 10 (ADAM-10) as novel binding partner of VEGFR-2. ADAM-10 is a metalloprotease with sheddase activity involved in cell migration, however, its exact function in endothelial cells (EC), angiogenesis and atherosclerosis is largely unknown. We show for the first time ADAM-10 expression in human atherosclerotic lesions, associated with plaque progression and neovascularization. We demonstrate ADAM-10 expression and activity in EC to be induced by VEGF and ADAM-10 to mediate ectodomain shedding of VEGFR-2. Furthermore, VEGF induces ADAM-10-mediated cleavage of VE-cadherin, which could increase vascular permeability and facilitate EC migration. Indeed, VEGF increases vascular permeability in an ADAM-10- and ADAM-17-dependent way and inhibition of ADAM-10 reduces EC migration and chemotaxis. These data provide the first evidence of ADAM-10 expression in atherosclerosis and neovascularization. ADAM-10 plays a functional role in VEGF-induced EC function. These data open perspectives for novel therapeutic interventions in vascular diseases.
\end{abstract}




\section{Introduction}

Angiogenesis is associated with tumor growth, metastasis, and with the growth and instability of atherosclerotic plaques. The latter contributes to plaque rupture and its clinical complications ${ }^{1}$. Expression of the major angiogenic factor Vascular Endothelial Growth Factor-A (VEGF) increases during atherogenesis. In EC, VEGF exerts its stimulatory effect mostly via VEGFR-2 (KDR/Flk-1) and is a potent angiogenic mediator of EC migration, proliferation and survival ${ }^{2}$. VEGF binding induces VEGFR-2 dimerization followed by (auto)phosphorylation on tyrosine residues. Activation of VEGFR-2 induces signaling via PLC $\gamma$, activation of the MAPK/ERK1/2-pathway and EC proliferation. Furthermore, activation of p38-MAPK or PI3K leads to EC migration, survival and increased permeability ${ }^{2,3}$.

Besides angiogenesis, VEGF and its receptors are associated with development of atherosclerosis, plaque angiogenesis and plaque instability. Recently, it was suggested that VEGF may induce a more vulnerable plaque phenotype by promoting leukocyte recruitment ${ }^{4}$. DNA vaccination against VEGFR-2 in LDLr-/- mice inhibited atherosclerotic plaque growth by reducing plaque neovascularization ${ }^{5}$. Treatment with a neutralizing anti-VEGFR-1 antibody reduced atherosclerosis in ApoE-/- mice via inhibition of leukocyte infiltration, whereas anti-VEGFR-2 was ineffective ${ }^{6}$. The neutralizing VEGF-antibody bevacizumab (Avastin ${ }^{\mathrm{TM}}$ ) is an effective anti-cancer therapy that inhibits tumor angiogenesis, but is associated with an increase in cardiovascular side effects based on thrombosis and plaque instability ${ }^{7}$. Unraveling downstream VEGFR signaling mechanisms involved in angiogenesis and atherosclerosis will identify novel therapeutic targets and will therefore open perspectives for more specific and more effective therapeutic interventions.

In this study we aimed to identify novel binding partners of VEGFR-2 involved in downstream VEGFR signaling by using a yeast-two-hybrid screening. We identified ADAM-10, a metalloprotease with alpha-secretase activity, as a novel candidate interacting with VEGFR-2. ADAM-10 is required for cleavage of membrane proteins involved in adhesion, migration and chemotaxis and has been implicated in cancer progression $^{8}$. ADAM-10-deficient mice are embryonically lethal due to defects in cardiovascular and neural development ${ }^{9}$.

Our data show for the first time a functional interaction between VEGFR-2 and ADAM-10, and a role of ADAM-10 in (VEGF-induced) endothelial cell function. In addition, we provide initial evidence for the expression of ADAM-10 in human atherosclerotic plaques, associated with neovascularization. 


\section{Methods}

Co-immunoprecipitation and in vitro kinase assay

These assays, combined with SDS-PAGE, were performed as previously described ${ }^{2}$ with minor modifications. For details, see supplement.

Fluorogenic peptide cleavage assay

EC were cultured in a 96-well plate and serum starved overnight once 70-90\% confluency was reached. Cells were pre-treated for $30 \mathrm{~min}$ with $10 \mu \mathrm{mol} / \mathrm{L}$ LY294002, PD98059 or SB203580. Medium was replaced by Hanks' Balanced salt solution (Sigma) containing $10 \mu \mathrm{mol} / \mathrm{L}$ Mca-PLAQAV-Dpa-RSSSR-NH2 fluorogenic peptide substrate (R\&D systems, ESO03). EC were stimulated with 5 or $50 \mathrm{ng} / \mathrm{mL}$ VEGF, $20 \mathrm{ng} / \mathrm{ml}$ PMA or $5 \mu \mathrm{mol} / \mathrm{L}$ ionomycin in presence of $5 \mu \mathrm{mol} / \mathrm{L} \mathrm{GI54023X,} 5$ $\mu \mathrm{mol} / \mathrm{L}$ GW280264X or dimethyl sulfoxide (DMSO). Increase of fluorescence was measured at $405 \mathrm{~nm}$ (excitation at $320 \mathrm{~nm}$ ) for $6 \mathrm{hrs}$ using a fluorescence microplate reader.

\section{Statistical Analysis}

Values are expressed as mean \pm SEM of at least $n=3$ experiments and a two-tailed unpaired Student's t-test was used to compare groups. Probability values $<0.05$ were considered significant.

For an extensive description of methods see supplements page 64. 


\section{Results}

Identification of ADAM-10 as novel VEGFR-2 binding partner

Using a yeast-two-hybrid screening with the intracellular domain of human VEGFR-2 as bait, we aimed to identify novel binding partners involved in downstream VEGFR2 mechanisms. Five screens were performed using a cDNA library of human placenta as angiogenic tissue. As depicted in table I (Supplement), a total of 93 out of 990 clones were found positive and were re-transfected for confirmation of the interaction, which yielded 20 candidates for interaction with VEGFR-2. Sequence analysis of these clones revealed A Disintegrin And Metalloprotease 10 (ADAM-10) as novel binding partner of VEGFR-2, with sequence coverage of $94 \%$ and identity of $100 \%$. As positive controls for our screening, NCK adaptor protein and PI3K were found, both proteins known to be involved in VEGFR-2 signaling ${ }^{2,10}$.

ADAM-10 is expressed in human atherosclerotic lesions and associated with angiogenesis

Since a role of ADAM-10 in vascular function and disease was largely unknown, we first investigated ADAM-10 expression in human vascular wall and atherosclerotic plaques using immunohistochemical staining. ADAM-10 expression is low in normal vessel wall (mammary artery, supplementary figure IA) and early human atherosclerotic lesions (Figure $1 A, n=5$ ) but significantly increased during plaque progression from early and advanced to ruptured atherosclerotic plaques (Figure 1B-C, $n=7-8$ ), as was determined by semi-quantitative analysis (table II, supplement) and confirmed by quantitative PCR analysis of human atherosclerotic plaque specimen $(p<0.01, n=6-7$, Figure 1D). ADAM-10 was expressed in most cell types, i.e. smooth muscle cells (SMC) and macrophages, especially in foam cells (Figure 1E) and most interestingly highly expressed in EC of plaque microvessels (Figure 1F), as confirmed by double staining for ADAM-10 and $C D 31^{+} / \mathrm{CD} 34^{+} \mathrm{EC}$ (Suppl. Figure IB). Additionally, we demonstrated high expression of ADAM-10 in human colon cancer cells as well as in the microvessels within, but not surrounding the colon tumor (Figure $1 \mathrm{G}-\mathrm{H}, \mathrm{n}=5$ and supplementary table III). These data further strengthen our finding of a functional association between ADAM-10 and angiogenesis. 

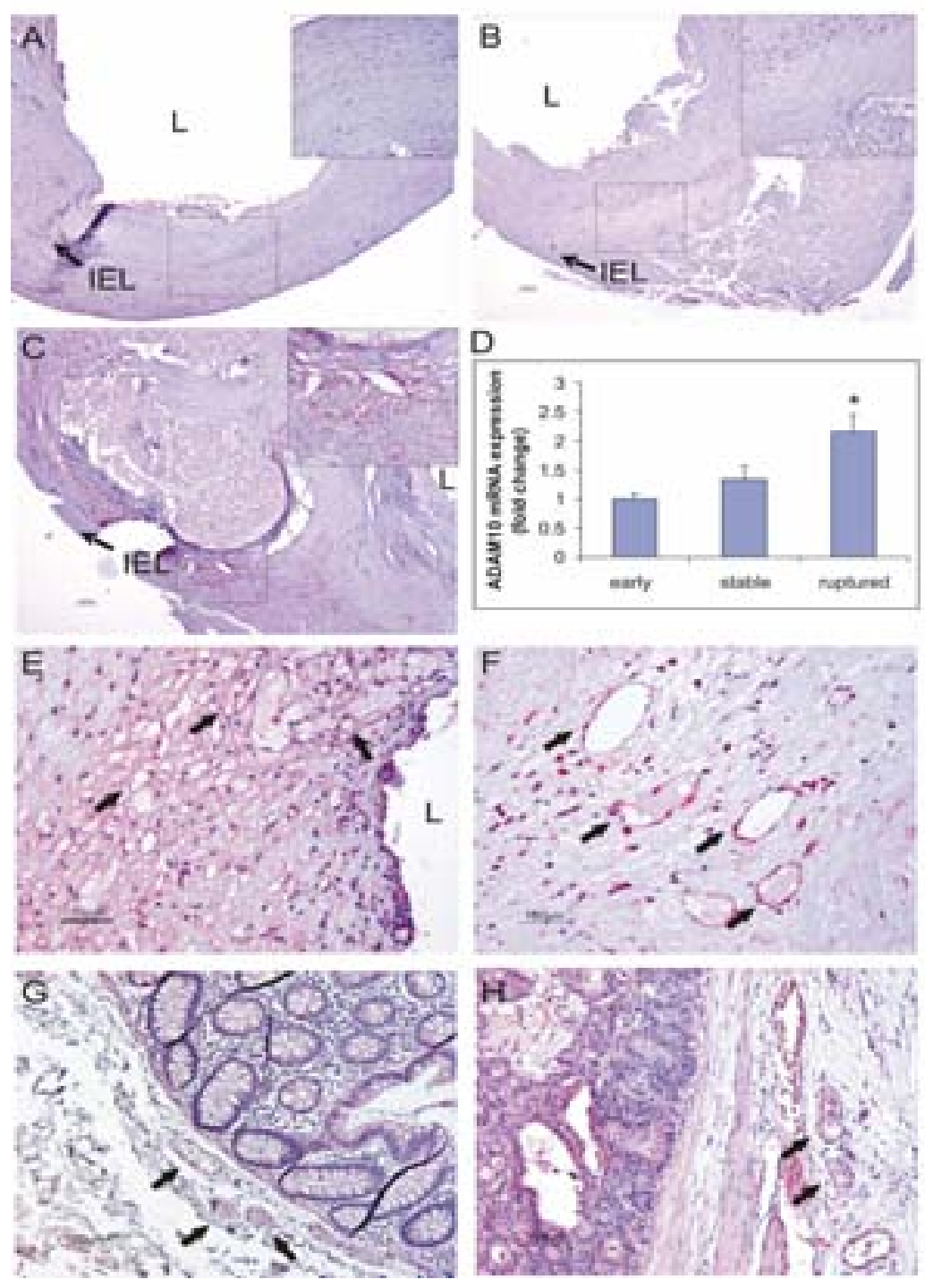

Figure 1. Immunohistochemical staining of ADAM-10 in human early (A), advanced stable (B) and ruptured $(C)$ atherosclerotic plaques, quantified by PCR (D, normalized to GAPDH). High expression was found in foam cells (E) and microvessels (F). L=lumen, IEL=internal elastic lamina. ADAM-10 expression in normal human colon $(\mathrm{G})$ and carcinoma with microvessels $(\mathrm{H})$.

ADAM-10 interacts with VEGFR-2 and mediates VEGFR-2 ectodomain shedding To validate the interaction of VEGFR-2 and ADAM-10 as identified in our yeast-twohybrid screening, we performed co-immunoprecipitation experiments. VEGFR-2 or ADAM-10 were precipitated from Porcine Aortic Endothelial Cells overexpressing human VEGFR-2 (PAEC-KDR) or untransfected PAEC stimulated with $0-50 \mathrm{ng} / \mathrm{mL}$ 
VEGF. Tyrosine-phosphorylated proteins were detected in precipitated immune complexes using an in vitro tyrosine kinase assay and separation of protein complexes by SDS-PAGE. In VEGFR-2 precipitates of PAEC-KDR, a strongly phosphorylated protein corresponding to the size of human VEGFR-2 ( $250 \mathrm{kD}$ ) was detected, which was absent from untransfected PAEC that do not express detectable levels of endogenous VEGFR-2 (Figure 2A). As expected for VEGFR-2, phosphorylation of this protein significantly increased after VEGF stimulation, strongly indicating this is indeed VEGFR-2. In ADAM-10 precipitates, several phosphorylated proteins could be detected. In unstimulated PAEC-KDR, phosphorylated VEGFR-2 could barely be detected, whereas after VEGF stimulation phosphorylated VEGFR-2 was clearly detectable as a co-precipitate of ADAM-10, supporting our Yeast-2-hybrid results of VEGFR-2 and ADAM-10 interaction. Since VEGFR-2 expression in primary human EC is much lower than in PAEC-KDR, VEGFR-2 co-immunoprecipitating with ADAM-10 in these cells was below detection limit (data not shown).

ADAM-10 is involved in the juxtamembrane cleavage of several cell surface molecules and receptors, thereby releasing their ectodomains (shedding) ${ }^{11}$. Since the related metalloprotease ADAM-17 has been implicated in VEGFR-2 shedding, we examined whether the interaction between ADAM-10 and VEGFR-2 provides a basis for ADAM-10-mediated ectodomain shedding of VEGFR-2. PAEC-KDR cells were incubated for $5 \mathrm{hrs}$ with two hydroxamate-based inhibitors of ADAM-10. The inhibitor GI254023X has been shown to block ADAM-10 with a more than 100-fold higher potency compared to ADAM-17, whereas compound GW280264X has been shown to block both ADAM-10 and ADAM-17 ${ }^{12}$. Inhibition of ADAM-10 by $5 \mu \mathrm{mol} / \mathrm{L}$ GI254023X, a concentration that selectively blocks ADAM-10 activity, as well as inhibition of both ADAM-10 and ADAM-17 by GW280264X significantly reduced constitutive release of VEGFR-2 ectodomain in PAEC-KDR culture medium compared to DMSO-treated controls (Figure 2B-C, $\mathrm{p}<0.001$ ). Release of sVEGFR-2 slightly increased after VEGF stimulation, which was again significantly decreased after inhibition of ADAM-10 only or both ADAM-10 and ADAM-17. Since PAEC-KDR cells overexpress the human VEGFR-2 and the proportion shed by the (limited) amount of ADAM-10 available in these cells will be limited, no differences in amount of full length VEGFR-2 in PAEC-KDR lysate were found (Figure 2B, lower panel). 


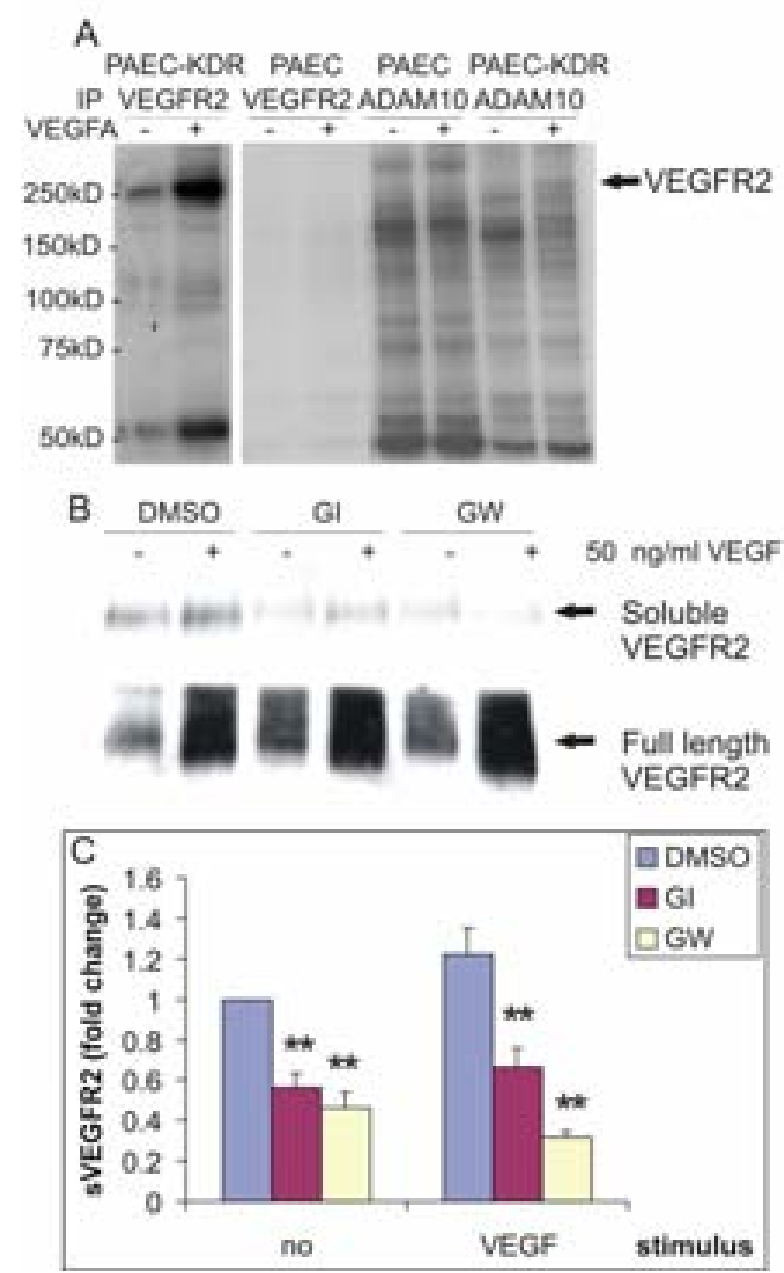

Figure 2. (A) Co-immunoprecipitation and in vitro kinase detection of VEGFR-2 of PAEC and PAEC-KDR. (B) Representative Western blot of soluble and full length VEGFR-2 in PAEC-KDR. ADAM-10 inhibition (GI254023X, $5 \mu \mathrm{mol} / \mathrm{L}$ ) or ADAM-10 and ADAM-17 inhibition (GW280264X, $5 \mu \mathrm{mol} / \mathrm{L}$ ) inhibits both constitutive and VEGF-induced VEGFR-2 shedding (B). ** $\mathrm{p}<0.001$

VEGF induces ADAM-10 expression in endothelial cells

Besides a physical interaction we examined the functional interaction between ADAM-10 and the VEGFR-2 pathway to determine whether ADAM-10 is involved in downstream VEGFR-2 functions. VEGF increased ADAM-10 mRNA expression up to $34 \%$ in PAEC-KDR (data not shown) and HUVEC (Suppl. figure II, $\mathrm{p} \leq 0.01$ ). Besides mRNA, we analyzed VEGF-induced ADAM-10 protein expression in ECs. In HUVECs ADAM-10 is expressed as a $\sim 135 \mathrm{kD}$ pro-enzyme which is processed into a $\sim 90 \mathrm{kDa}$ mature and active enzyme (Figure $3 A$ and B). VEGF stimulation of HUVECs for $24 \mathrm{hrs}$ 
significantly induced total ADAM-10 protein expression up to 1.95 fold (figure 3C, $\mathrm{p}<0.01)$. Most interestingly, short-term stimulation with VEGF resulted in a 2.1 fold increase of processed, mature i.e. active ADAM-10 in HUVECs (Figure 3D, $p<0.01$ ). Similar results were found in PAEC-KDR (data not shown). These data indicate that VEGF stimulation of EC not only induces total ADAM-10 expression at both the mRNA and protein level, but could also induce the processing of the latent enzyme into the activate ADAM-10 protein.

\section{ADAM-10 activity in endothelial cells is induced by VEGF}

To confirm ADAM-10 activation by VEGF stimulation in EC, we used a fluorogenic peptide as a substrate, which emits a fluorescent signal when degraded. As expected, PMA and ionomycin induced peptide degradation in HUVEC. Stimulation with $50 \mathrm{ng} / \mathrm{ml}$ VEGF significantly induced peptide degradation in 6 hrs by 1.4 fold in HUVECS (Figure $3 E, p<0.05$ ). Since this peptide can also be degraded by ADAM-17 and ADAM9, the inhibitors GI254023X and GW280264X were used to determine the contribution of ADAM-10 to VEGF-induced peptide degradation. Inhibition of ADAM-10 alone by GI254023X reduced basal peptide degradation by $10 \%(p=0.08)$. Interestingly, VEGF-induced peptide degradation was not significantly stimulated anymore when ADAM-10 was inhibited by GI235024X (Figure 3E). Combined ADAM10 and ADAM-17 inhibition by GW280264X reduced basal and VEGF-induced peptide degradation ( $18 \%, \mathrm{p}<0.01$ and $41 \%, \mathrm{p}<0.001$ respectively). Similar results were found in PAEC-KDR (data not shown).

VEGF stimulates several signaling pathways by VEGFR-2 activation ${ }^{2,3}$. Therefore we used specific inhibitors to identify the signaling pathways required for VEGF-induced ADAM-10-activity. In non-stimulated HUVEC, inhibition of PI3K, p38 and ERK1/2 activation slightly reduced basal peptide degradation but not significantly. Interestingly, VEGF-dependent increase of peptide degradation was significantly inhibited by ERK1/2 inhibition $(p<0.05)$. Similar results were found by PI3K inhibition, while no significant effects were seen with p38 inhibition (Supplementary figure III). These data indicate that VEGF induces ADAM-10 (and ADAM-17) activity in ECs via ERK1/2 and potentially PI3K signaling. 

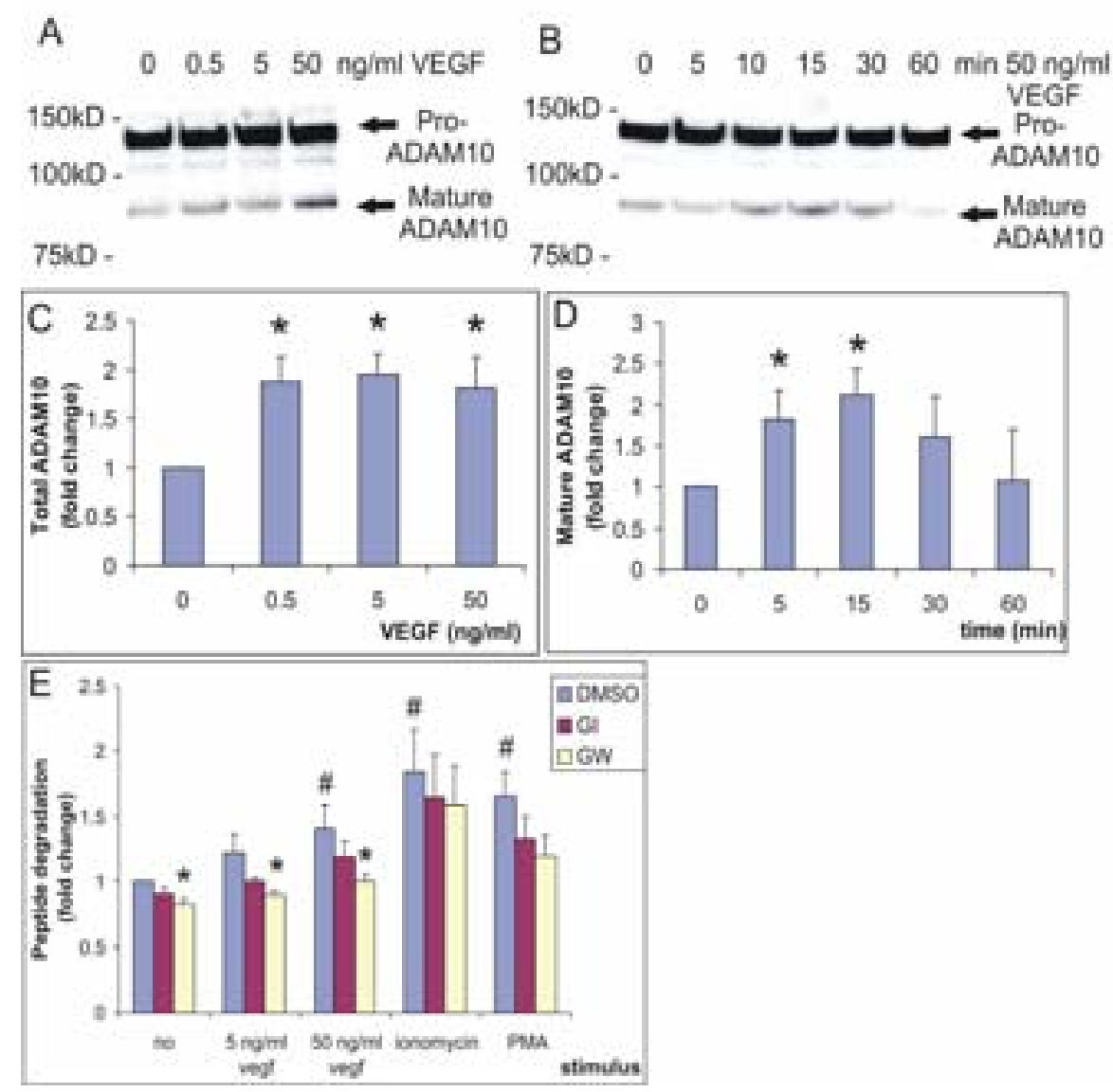

Figure 3. VEGF increases HUVEC total protein ( $A-C)$ and mature ADAM-10 expression (B-D, * $p<0.05$ ). (E) VEGF-induced fluorogenic peptide degradation ( $6 \mathrm{hrs}$; $\# p<0.05$ vs DMSO without stimulus) is inhibited by GI254023X ( $5 \mu \mathrm{mol} / \mathrm{L})$. GW280264X (5 $\mu \mathrm{mol} / \mathrm{L})$ reduces basic and VEGF-induced peptide degradation $\left({ }^{*} p<0.05\right)$.

VEGF induces ADAM-10-mediated VE-cadherin cleavage, thereby increasing permeability

ADAM-10 can cleave several adhesion molecules, e.g. $\mathrm{N}$-cadherin, E-cadherin and the endothelial specific VE-cadherin ${ }^{13}$. Cleavage of VE-cadherin was shown to increase endothelial permeability and T-cell transmigration, two important processes in angiogenesis and atherosclerosis. To investigate whether VEGF could induce this functional activity of ADAM-10, we stimulated HUVEC for 24 hrs with VEGF in presence or absence of GI254023X or GW280264X. The $\gamma$-secretase inhibitor DAPT (10 $\mu \mathrm{mol} / \mathrm{L})$ was used to prevent further intracellular processing of VE-cadherin after initial cleavage by ADAM-10, allowing quantification of the cleaved intracellular fragment (Figure 4A). Both GI254023X and GW280264X 
completely blocked cleavage of VE-cadherin, confirming indeed the role of ADAM10 in cleavage of this EC adhesion molecule (Figure 4B). Interestingly, VEGF stimulation increased VE-cadherin cleavage by $41 \%(p=0.02)$, which was prevented by ADAM-10 inhibition ( $p<0.01$ ). Results of pharmacological inhibition of ADAM-10 and ADAM-17 on VE-cadherin cleavage were confirmed using an RNAi approach. HUVEC transfection with specific ADAM-10 siRNAs resulted in specific knockdown of ADAM-10 protein (70-80\%) without affecting ADAM-17 expression (Supplementary figure IV). Similarly, specific ADAM-17 knockdown (50-60\%) was found without a significant effect on ADAM-10 expression. Interestingly, specific knockdown of ADAM-10 significantly inhibited VE-cadherin cleavage in HUVEC $60 \%$ reduction, $p<0.0001$ for unstimulated HUVEC, Figure 4C-D). ADAM-17 knockdown in HUVEC did not block VE-cadherin. This indicates the involvement of ADAM-10, but not ADAM-17 in VE-cadherin cleavage in HUVEC.

Since ADAM-10 mediated VE-cadherin cleavage was shown to increase vascular permeability and leukocyte transmigration, we investigated whether VEGF could mediate its effect on vascular permeability by ADAM-10. HUVEC were grown to confluency and electrical resistance was measured using the Electric Cell-substrate Impedance Sensing (ECIS) system. Electrical resistance of HUVEC without VEGFtreatment remained constant throughout the experiment (data not shown). As expected, after a transient increase in resistance, VEGF induced a gradual decline in cellular resistance corresponding to an increased permeability (Figure 4E). A similar VEGF-induced decline in resistance was found when only ADAM-10 was inhibited by GI254023X. However, combined inhibition of ADAM-10 and ADAM-17 by GW280264X attenuated the VEGF-induced decline in resistance (Figure 4E). This indicates that, whereas ADAM-10 inhibition alone is sufficient to block VE-cadherin cleavage, VEGF-induced EC permeability is regulated by both ADAM-10 and ADAM17 , probably involving cleavage of other cell-cell adhesion molecules besides VEcadherin. 


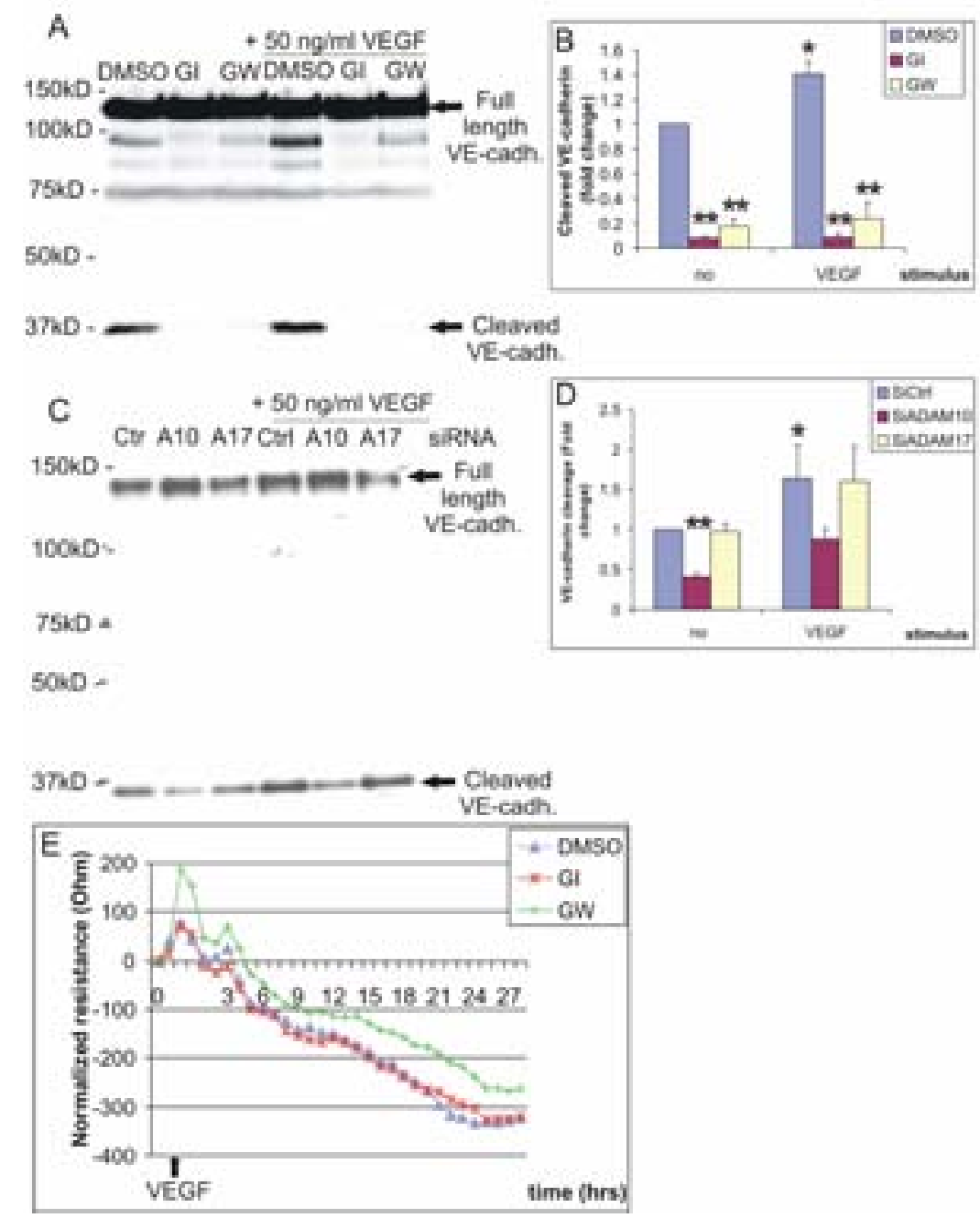

Figure 4. VEGF induces VE-cadherin cleavage in HUVEC, which is completely blocked by GI254023X or GW280264X ( $5 \mu \mathrm{mol} / \mathrm{L}, \mathrm{A}-\mathrm{B}$ ) or ADAM-10 siRNA, but not ADAM-17 siRNA (C-D). (E) ECIS permeability measurement of HUVEC pre-treated with $5 \mu \mathrm{mol} / \mathrm{L} \mathrm{GI} 254023 X$, GW280264X or DMSO followed by 100 $\mathrm{ng} / \mathrm{ml}$ VEGF stimulation. ${ }^{*} \mathrm{p}<0.05,{ }^{* *} \mathrm{p}<0.01, \mathrm{n}=3$.

ADAM-10 is required for endothelial cell and monocyte migration and chemotaxis Since cleavage of adhesion molecules is essential for cell migration, we examined the requirement of ADAM-10 for EC migration and chemotaxis using a Boyden chamber. Baseline EC migration (chemokinesis) was significantly reduced (39\%) when ADAM-10 was inhibited by GI254023X, whereas VEGF-induced chemotaxis was reduced by $41 \%$ (Figure $5 \mathrm{~A}, \mathrm{p}=0.005$ and $\mathrm{p}=0.02$, respectively). Besides $E C$, monocytes are critical players in cardiovascular disease, not only in atherosclerosis, 
but also by participating in neovascularization ${ }^{14}$. ADAM-10 inhibition also significantly reduced both monocyte chemokinesis (50\%, Figure $5 \mathrm{~B}$ ) and chemotaxis (52\% for VEGF and $36 \%$ for $\mathrm{FMLP}$ ), indicating that ADAM-10 is required for cell migration, most likely by facilitating cell detachment by cleavage of cell adhesion molecules.

Using Electric Cell-substrate Impedance Sensing (ECIS) we measured the migratory healing response of HUVEC after wounding by a high electrical field. In control cells, resistance returned to steady state conditions within $8 \mathrm{hrs}$ after wounding, indicating that the response is rather a result of EC migration than proliferation (Figure 5C). Inhibition of ADAM-10 by GI254023X delayed this wound healing response which was even more pronounced when both ADAM-10 and ADAM-17 were inhibited by GW280264X.
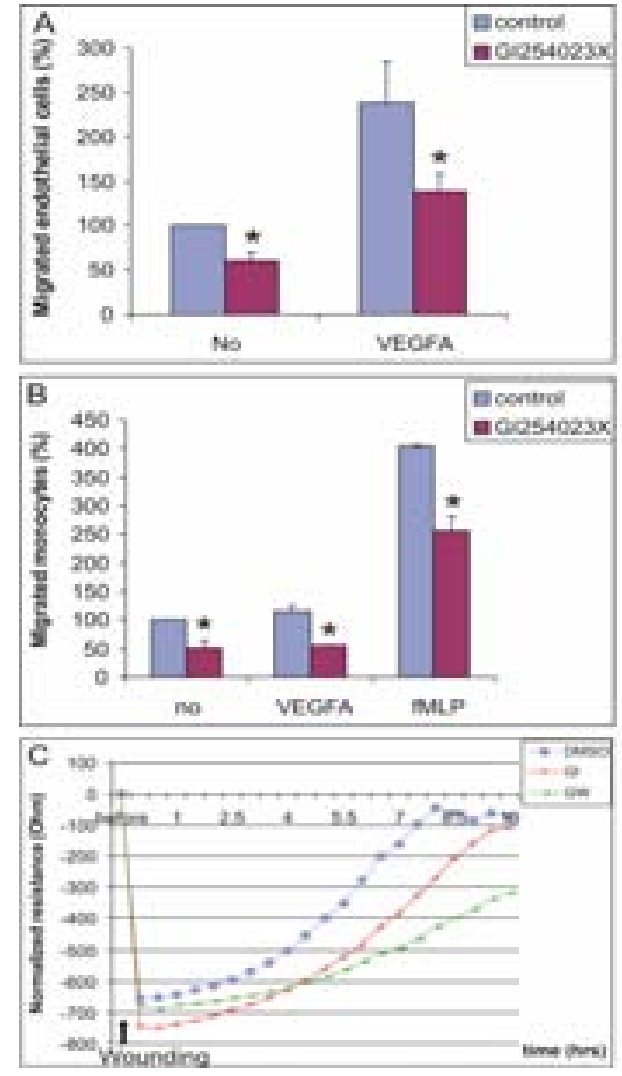

Figure 5. (A) GI254023X $(5 \mu \mathrm{mol} / \mathrm{L})$ reduces PAECKDR migration and chemotaxis towards 10 $\mathrm{ng} / \mathrm{ml} \quad$ VEGF. (B) GI254023X reduces monocyte migration and chemotaxis towards 10 $\mathrm{ng} / \mathrm{ml}$ VEGF and $10^{8}$ $\mathrm{mol} / \mathrm{L} \quad \mathrm{fMLP} . \quad{ }^{*} \mathrm{p}<0.05$, $\mathrm{n}=3$. (C) GI254023X and GW280264X (5 $\mu \mathrm{mol} / \mathrm{L})$ reduces HUVEC migration response after applying a high electrical field. 


\section{Discussion}

In this study we identified ADAM-10 as a novel mediator of VEGF-induced EC function and provide first evidence of ADAM-10 interaction with VEGFR-2. VEGF induces both ADAM-10 expression and activity. We showed ADAM-10 to be required for VEGF-induced VE-cadherin cleavage, vascular permeability and EC migration. This is the first report to document ADAM-10 expression in human atherosclerotic lesions, associated with atherosclerotic plaque progression and neovascularization.

Using a yeast-two-hybrid screening we identified ADAM-10 as novel binding candidate for VEGFR-2, which was supported by co-immunoprecipitation of VEGFR2 with ADAM-10 in PAEC-KDR. This interaction could be of functional significance in endothelial biology. Recently, Swendemann et al. showed the highly related ADAM17 to mediate VEGFR-2 shedding, which could be induced by VEGF ${ }^{15}$. For ADAM-10 they reported a role in shedding of neuropilin-1, the co-receptor for VEGFR-2. In this study, we showed not only ADAM-17, but also ADAM-10 to be involved in VEGFR-2 ectodomain shedding. Shedding of VEGFR-2 and neuropilin-1 could provide a mechanism to modulate VEGFR-2-signaling, e.g. as a negative feedback loop to dampen the VEGF stimulatory signal. Such an interaction would be transient, which might also explain why we could only find a small amount of VEGFR-2 coprecipitating with ADAM-10.

Besides a physical interaction, we demonstrated a functional interaction between ADAM-10 and the VEGFR-2-pathway. We found VEGF to induce not only ADAM-10 expression, but more importantly, to stimulate its proteolytic activity. The exact mechanism of activation of ADAM-10 by VEGF remains to be elucidated. In this study, we found evidence of ERK1/2 involvement in VEGF-induced peptide degrading activity of ADAM-10 (although the involvement of ADAM9 and ADAM-17 can not be excluded in this assay). VEGF is known to increase intracellular calcium levels by activating PLC ${ }^{16}$. Increased intracellular calcium has been shown to induce ADAM-10 processing (i.e. activation) by furin-like proteases ${ }^{17}$. Whether induction of ADAM-10 activity by VEGF is indeed dependent on calcium influxes and activity of furin or other proteases remains to be determined.

In this study, we showed VEGF to stimulate cleavage of the endothelial specific adhesion molecule VE-cadherin by ADAM-10, but not ADAM-17, using both pharmacological inhibitors and specific siRNAs for ADAM-10 and ADAM-17. Recently, VE-cadherin cleavage has been shown to increase EC permeability and T cell transmigration ${ }^{18}$. VEGF is well known to induce endothelial permeability by promoting phosphorylation, endocytosis of VE-cadherin and subsequent disruption of adhe-rens junctions ${ }^{19}$. Our data clearly demonstrate a role for ADAM-10, but also ADAM-17, in VEGF-induced EC permeability. Whereas VE-cadherin cleavage was nearly completely abrogated when only ADAM-10 was inhibited, VEGF-mediated HUVEC permeability was only attenuated by inhibition of both ADAM-10 and 
ADAM-17. This might be explained by ADAM-10/17-mediated cleavage of other EC junctional adhesion molecules that are involved in regulating endothelial permeability. Recently, Koenen et al. showed that cleavage of Junctional Adhesion Molecule-1 (JAM-1) in EC to be predominantly mediated by ADAM-17 and to a lesser extent by ADAM-10 $0^{20}$. Since JAM-1 is also involved in regulating endothelial permeability $^{21}$, this might explain why it is necessary to inhibit both ADAM-10 and ADAM-17 to effectively block VEGF-mediated increase in permeability, even though ADAM-10 inhibition alone is sufficient to block cleavage of individual adhesion molecules, i.e. VE-cadherin. Increased EC permeability has also been implicated in progression and rupture of atherosclerotic lesions, where the microvasculature has been proposed to be immature and 'leaky" ${ }^{22}$. Consequently, leukocytes can transmigrate through the endothelium more easily and enter the atherosclerotic plaque, where they can contribute to plaque progression and destabilization.

Besides adhesion molecules, ADAM-10 is known to be involved in shedding of numerous chemotactic and inflammatory molecules such as CXCL16, fractalkine, IL6 receptor, TNF $\alpha, \mathrm{RAGE}$ and $\mathrm{CD}_{40 \mathrm{~L}^{23}}$, all molecules known to contribute to atherogenesis and plaque progression. It is therefore not surprising that we found increased ADAM-10 expression in advanced human atherosclerotic plaques with a high number of infiltrating cells and a high degree of inflammation. Furthermore, we found high ADAM-10 expression associated with plaque neovascularization as well as tumor neovascularization. ADAM-10 has been implicated in the proteolytic cleavage of several proteins regulating arteriogenic and angiogenic processes, e.g. members of the Notch pathway ${ }^{24}$ and Ephrin (ligands) ${ }^{25}$. Recently, VEGF was shown to induce shedding of the angiopoietin receptor Tie-2, which likely is mediated by ADAM- $10^{26}$. Furthermore, VEGF-induced Notch activation in EC was ADAM-10 dependent (own, unpublished data). Activation of Notch has been shown to be involved in the regulation of vessel sprouting ${ }^{27}$, which, besides inhibiting EC migration, provides another mechanism by which ADAM-10 inhibition could modulate neovascularization responses. These functional data of a role of ADAM-10 in (VEGF-induced) endothelial cell functions, together with our finding of a direct interaction between ADAM-10 and VEGFR pathway, which by shedding of the VEGFR-2 could modulate VEGFR-2-signaling and downstream endothelial cell functions, strengthen the hypothesis that ADAM-10 plays a crucial role in various mechanisms underlying angiogenic and atherosclerotic processes. Furthermore, it may also have important implications for other diseases in which neovascularization contributes to disease progression such as cancer.

Besides ADAM-10, other ADAM proteins may be involved in the angiogenic and atherosclerotic processes. As mentioned above, ADAM-17 is related to ADAM-10 and shares several substrates. Nevertheless, differences in regulation between ADAM-10 and ADAM-17 functions have been reported, i.e. in pathways leading to activation of the proteases, in constitutive versus regulated proteolytic activity and 
substrate specificity ${ }^{13,15,28}$. Interestingly, ADAM-17 expression has been associated with both atherosclerotic lesion progression and resistance to atherosclerosis in mice $^{29,30}$. Recently, ADAM-17 was also shown to be involved in endothelial tube formation in vitro and pathological neovascularization in vivo ${ }^{31}$. Pathological retinal neovascularization and growth of heterotopically injected tumor cells was reduced in mice that lack ADAM-17 specifically in the endothelial cells. Furthermore, ADAM15 has been shown to mediate inflammation and was found to be associated with atherosclerosis ${ }^{32}$. Therefore, it will be important to further elucidate the exact mechanisms of ADAM functions in cardiovascular diseases, and most challenging to determine specificity of ADAM-10 functions compared to related ADAMs. In this study, we used two approaches to inhibit ADAM-10 and ADAM-17 activities, i.e the hydroxamate-based compounds G1254023X and GW280264X, which were designed to discriminate between $A D A M-10$ and $A D A M-17^{12}$, and specific knockdown of either ADAM-10 or ADAM-17 using siRNA. Both approaches showed similar results. However, to identify the involvement of other distinct ADAM proteases in (VEGFinduced) endothelial cell functions additional selective inhibitors are needed. Furthermore, it will be an important task to determine the role of ADAM-10 in neovascularization and cardiovascular diseases in vivo. Since ADAM-10-/- mice are embryonically lethal ${ }^{9}$, this will require the use of conditional knockout models.

In conclusion, this study shows - for the first time - expression of ADAM-10 in atherosclerotic lesions, associated with neovascularization. This provides the first evidence of an interaction between the VEGFR-2 pathway and ADAM-10, and a functional role of ADAM-10 in (VEGF-induced) endothelial cell functions. Therefore these data open perspectives for novel therapeutic interventions in neovascularization and vascular diseases.

\section{Acknowledgments}

We thank Dr. Judy van Beijnum for providing HUVECs, Floris van Alphen for technical support and Dr. David Becherer, GlaxoSmithKline, for providing GI254023X and GW280264X.

This work was supported by grants from IZKF UIm, University of UIm (J.W.), the European Community's Sixth Framework Programme integrated project ANGIOTARGETING contract no 504743 (J.W.) and the Dutch Heart Foundation (Dr. E. Dekker grant 2007T034 to M.M.P.C.D.). J.D.v.B. and M.J.P. are supported by the Dutch Heart Foundation (Dr. E. Dekker grant 2005T039 and 2005B254, respectively). V.C. is supported by a MarieCurie Fellowship (MEST-CT-2005-020706). 


\section{References}

1. Virmani R, Kolodgie FD, Burke AP, Finn AV, Gold HK, Tulenko TN, Wrenn SP, Narula J. Atherosclerotic plaque progression and vulnerability to rupture: angiogenesis as a source of intraplaque hemorrhage. Arterioscler Thromb Vasc Biol. 2005;25:2054-2061.

2. Waltenberger J, Claesson-Welsh L, Siegbahn A, Shibuya M, Heldin CH. Different signal transduction properties of KDR and Flt1, two receptors for vascular endothelial growth factor. $J$ Biol Chem. 1994;269:26988-26995.

3. Olsson AK, Dimberg A, Kreuger J, Claesson-Welsh L. VEGF receptor signalling - in control of vascular function. Nat Rev Mol Cell Biol. 2006;7:359-371.

4. Celletti FL, Waugh JM, Amabile PG, Brendolan A, Hilfiker PR, Dake MD. Vascular endothelial growth factor enhances atherosclerotic plaque progression. Nat Med. 2001;7:425-429.

5. Petrovan RJ, Kaplan CD, Reisfeld RA, Curtiss LK. DNA vaccination against VEGF receptor 2 reduces atherosclerosis in LDL receptor-deficient mice. Arterioscler Thromb Vasc Biol. 2007;27:1095-1100.

6. Luttun A, Tjwa M, Moons L, Wu Y, Angelillo-Scherrer A, Liao F, Nagy JA, Hooper A, Priller J, De Klerck B, Compernolle V, Daci E, Bohlen P, Dewerchin M, Herbert JM, Fava R, Matthys P, Carmeliet G, Collen D, Dvorak HF, Hicklin DJ, Carmeliet P. Revascularization of ischemic tissues by PIGF treatment, and inhibition of tumor angiogenesis, arthritis and atherosclerosis by anti-Flt1. Nat Med. 2002;8:831-840.

7. Ratner M. Genentech discloses safety concerns over Avastin. Nat Biotechnol. 2004;22:1198.

8. Moss ML, Stoeck A, Yan W, Dempsey PJ. ADAM10 as a target for anti-cancer therapy. Curr Pharm Biotechnol. 2008;9:2-8.

9. Hartmann D, de Strooper B, Serneels L, Craessaerts K, Herreman A, Annaert W, Umans L, Lubke T, Lena Illert A, von Figura K, Saftig P. The disintegrin/metalloprotease ADAM 10 is essential for Notch signalling but not for alpha-secretase activity in fibroblasts. Hum Mol Genet. 2002;11:2615-2624.

10. Kroll J, Waltenberger J. The vascular endothelial growth factor receptor KDR activates multiple signal transduction pathways in porcine aortic endothelial cells. J Biol Chem. 1997;272:32521-32527.

11. Deuss M, Reiss K, Hartmann D. Part-time alpha-secretases: the functional biology of ADAM 9,10 and 17. Curr Alzheimer Res. 2008;5:187-201.

12. Ludwig A, Hundhausen C, Lambert MH, Broadway N, Andrews RC, Bickett DM, Leesnitzer MA, Becherer JD. Metalloproteinase inhibitors for the disintegrin-like metalloproteinases ADAM10 and ADAM-17 that differentially block constitutive and phorbol ester-inducible shedding of cell surface molecules. Comb Chem High Throughput Screen. 2005;8:161-171.

13. Hundhausen C, Misztela D, Berkhout TA, Broadway N, Saftig P, Reiss K, Hartmann D, Fahrenholz F, Postina R, Matthews V, Kallen KJ, Rose-John S, Ludwig A. The disintegrin-like metalloproteinase ADAM10 is involved in constitutive cleavage of CX3CL1 (fractalkine) and regulates CX3CL1-mediated cell-cell adhesion. Blood. 2003;102:1186-1195.

14. Arras M, Ito WD, Scholz D, Winkler B, Schaper J, Schaper W. Monocyte activation in angiogenesis and collateral growth in the rabbit hindlimb. J Clin Invest. 1998;101:40-50.

15. Swendeman S, Mendelson K, Weskamp G, Horiuchi K, Deutsch U, Scherle P, Hooper A, Rafii S, Blobel CP. VEGF-A stimulates ADAM17-dependent shedding of VEGFR2 and crosstalk between VEGFR2 and ERK signaling. Circ Res. 2008;103:916-918.

16. Faehling M, Kroll J, Fohr KJ, Fellbrich G, Mayr U, Trischler G, Waltenberger J. Essential role of calcium in vascular endothelial growth factor A-induced signaling: mechanism of the antiangiogenic effect of carboxyamidotriazole. Faseb J. 2002;16:1805-1807.

17. Anders A, Gilbert S, Garten W, Postina R, Fahrenholz F. Regulation of the alpha-secretase ADAM10 by its prodomain and proprotein convertases. Faseb J. 2001;15:1837-1839.

18. Schulz B, Pruessmeyer J, Maretzky T, Ludwig A, Blobel CP, Saftig P, Reiss K. ADAM10 regulates endothelial permeability and T-Cell transmigration by proteolysis of vascular endothelial cadherin. Circ Res. 2008;102:1192-1201.

19. Gavard J, Gutkind JS. VEGF controls endothelial-cell permeability by promoting the beta-arrestindependent endocytosis of VE-cadherin. Nat Cell Biol. 2006;8:1223-1234. 
20. Koenen RR, Pruessmeyer J, Soehnlein O, Fraemohs L, Zernecke A, Schwarz N, Reiss K, Sarabi A, Lindbom L, Hackeng TM, Weber C, Ludwig A. Regulated release and functional modulation of junctional adhesion molecule A by disintegrin metalloproteinases. Blood. 2009;113:4799-4809.

21. Weber C, Fraemohs L, Dejana E. The role of junctional adhesion molecules in vascular inflammation. Nat Rev Immunol. 2007;7:467-477.

22. Sluimer JC KF, Bijnens AP, Pacheco E, Frederik PM, Van Hinsbergh VWM, Virmani R, Daemen MJ. Infrequent pericyte coverage and clear structural abnormalities are present in intraplaque microvessels in human coronary atherosclerotic plaques. J. Am. Coll. Cardiol. 2009; In press.

23. Garton KJ, Gough PJ, Raines EW. Emerging roles for ectodomain shedding in the regulation of inflammatory responses. J Leukoc Biol. 2006;79:1105-1116.

24. Tian L, Wu X, Chi C, Han M, Xu T, Zhuang Y. ADAM10 is essential for proteolytic activation of Notch during thymocyte development. Int Immunol. 2008;20:1181-1187.

25. Janes PW, Saha N, Barton WA, Kolev MV, Wimmer-Kleikamp SH, Nievergall E, Blobel CP, Himanen JP, Lackmann M, Nikolov DB. Adam meets Eph: an ADAM substrate recognition module acts as a molecular switch for ephrin cleavage in trans. Cell. 2005;123:291-304.

26. Findley CM, Cudmore MJ, Ahmed A, Kontos CD. VEGF induces Tie2 shedding via a phosphoinositide 3-kinase/Akt dependent pathway to modulate Tie2 signaling. Arterioscler Thromb Vasc Biol. 2007;27:2619-2626.

27. Horowitz A, Simons M. Branching morphogenesis. Circ Res. 2008;103:784-795.

28. Le Gall SM, Bobe P, Reiss K, Horiuchi K, Niu XD, Lundell D, Gibb DR, Conrad D, Saftig P, Blobel CP. ADAMs 10 and 17 Represent Differentially Regulated Components of a General Shedding Machinery for Membrane Proteins such as TGF\{alpha\}, L-Selectin and TNF\{alpha\}. Mol Biol Cell. 2009.

29. Canault M, Peiretti F, Kopp F, Bonardo B, Bonzi MF, Coudeyre JC, Alessi MC, Juhan-Vague I, Nalbone G. The TNF alpha converting enzyme (TACE/ADAM17) is expressed in the atherosclerotic lesions of apolipoprotein E-deficient mice: possible contribution to elevated plasma levels of soluble TNF alpha receptors. Atherosclerosis. 2006;187:82-91.

30. Holdt LM, Thiery J, Breslow JL, Teupser D. Increased ADAM17 mRNA expression and activity is associated with atherosclerosis resistance in LDL-receptor deficient mice. Arterioscler Thromb Vasc Biol. 2008;28:1097-1103.

31. Weskamp G, Mendelson K, Swendeman S, Le Gall S, Ma Y, Lyman S, Hinoki A, Eguchi S, Guaiquil V, Horiuchi K, Blobel CP. Pathological neovascularization is reduced by inactivation of ADAM17 in endothelial cells but not in pericytes. Circ Res. 2010;106:932-940.

32. Charrier-Hisamuddin L, Laboisse CL, Merlin D. ADAM-15: a metalloprotease that mediates inflammation. Faseb J. 2008;22:641-653. 


\section{Supplement}

\section{Methods}

Compounds

Recombinant human VEGFA 165 (VEGF) was purchased from Reliatech. The metalloprotease inhibitors GI254023X and GW280264X were a kind gift from GlaxoSmithKline. Inhibitors LY294002, PD98059 and SB203580 were purchased from Sigma and $y$-secretase inhibitor IX (DAPT) was purchased from Calbiochem.

\section{Yeast-two-hybrid screening}

The nucleotide sequence corresponding to amino acids 790-1356 of the intracellular domain of human VEGFR-2 (VEGFR-2cyt) was amplified by PCR performed on the full length VEGFR-2 CDNA sequence. The following primers were used for PCR: sense primer: 5'-GGGTTTCATATGAAGCGGGCCAATGGAGGG-3' (Ndel restriction site) antisense primer: 5'-TGCGGTCGACTTAAACAGGAGGAGAGCTCAG-3' (Sall restriction site). As bait, this VEGFR-2cyt sequence was cloned between Ndel and Sall restriction sites into the pGTBKT7 plasmid and transformed into the AH109 yeast strain. A human placenta cDNA library was cloned into the PACT2 plasmid and transformed into the AH109 yeast strain according to the Matchmaker Gal4 TwoHybrid system 3 (Clontech). From $\beta$-galactosidase-positive clones, cDNA was isolated and retransformed together with VEGFR-2cyt-pGBKT7 into the AH109 yeast strain to confirm the interaction. Confirmed positive clones were selected, sequenced and aligned with the human genome using BLASTn (NCBI).

\section{Immunohistochemistry}

Formalin-fixed paraffin sections or RNA samples of human atherosclerotic plaques were obtained from the Maastricht Pathology Tissue Collection (MPTC). Collection, storage and use of tissue and patient data were performed in agreement with the 'Code for Proper Secondary Use of Human Tissue in the Netherlands'. Sections were (double) stained after antigen retrieval by boiling in $1 \mathrm{x}$ citrate $\mathrm{pH} 6$ antigen retrieval buffer (Dako) with anti-ADAM-10 antibody (Chemicon 19026, 1:20), followed by incubation with biotin-labeled swine anti-rabbit antibody (1:1000, Dako). ABCalkaline phosphatase and vector red/blue were used for visualization. For double stainings, sections were subsequently stained with anti-CD31 (1:50) combined with anti-CD34 (1:200) for EC and 1:200 biotin-labeled rabbit anti- mouse as secondary antibody. $A B C^{\text {hrp }}$ and diaminobenzidine (DAB) were used for visualization.

\section{Cell culture}

Porcine Aortic Endothelial Cells overexpressing human VEGFR-2 (PAEC-KDR) ${ }^{1}$ were grown in Ham's F-12 medium containing 10\% FCS and gentamycin G418 until 70- 
$90 \%$ confluency. Before stimulations, cells were serum starved overnight in Ham's F12 medium containing $0.01 \%$ BSA and G418.

Human Umbilical Vein Endothelial Cells (HUVECs) were obtained by trypsin perfusion of umbilical cords from the department of Obstetrics and Gynecology at the University Hospital Maastricht, as described previously ${ }^{2}$. Cells were grown on gelatin in RPMI medium containing 10\% FCS, $10 \%$ human serum and streptomycin/penicillin. Cells were serum starved in RPMI medium containing $1 \%$ FCS, $1 \%$ human serum and streptomycin/penicillin and used when subconfluent.

$R N A i$

HUVEC (70\% confluency) were transfected with $10 \mathrm{nM}$ of either ADAM-10 or ADAM17 ON-TARGET plus siRNA SMARTpools or non-targeting siRNA pool (Dharmacon) using Interferin (polyplus, Illkirch, France) following manufacturer's protocol. After $48 \mathrm{hrs}$ of transfection, cells were used for experiments. From all transfections, a fraction was lysed and analysed on Western blot for protein knockdown.

\section{Co-immunoprecipitation and in vitro kinase assay}

These assays were performed as previously described ${ }^{1}$ with minor modifications. After overnight starvation, PAEC-KDR and PAEC were pre-incubated with $100 \mu \mathrm{M}$ sodium orthovanadate for $10 \mathrm{~min}$ and stimulated with 0-50 $\mathrm{ng} / \mathrm{ml} \mathrm{VEGF} \mathrm{for} 3 \mathrm{~min}$. Cells were lysed at $4^{\circ} \mathrm{C}$ in $0.05 \%$ NP40 lysisbuffer (containing $150 \mathrm{mmol} / \mathrm{L} \mathrm{NaCl}, 20$ $\mathrm{mmol} / \mathrm{L}$ Tris pH 7.4, $10 \mathrm{mmol} / \mathrm{L}$ EDTA, 0.05\% NP40 and protease cocktail (Roche)). After pre-clearance, KDR or ADAM-10 were precipitated with $2.5 \mu \mathrm{g}$ rabbit-antihuman VEGFR-2 antibody (Santa Cruz C1158) or affinity-purified rabbit-anti-human ADAM-10 antibodies (A3226, Sigma), respectively. As negative control, precipitation without primary antibody was performed using equal amounts of protein. Immune complexes were precipitated for $45 \mathrm{~min}$ at $4^{\circ} \mathrm{C}$ with protein $A$ Sepharose beads (Amersham Biosciences) and incubated for $7 \mathrm{~min}$ at RT with $5 \mu \mathrm{Ci} / \mu{ }^{32} \mathrm{P}-\mathrm{\gamma}$-ATP in kinase buffer $\left(50 \mathrm{mmol} / \mathrm{L}\right.$ HEPES, $10 \mathrm{mmol} / \mathrm{L} \mathrm{MnCl}_{2}, 1 \mathrm{mmol} / \mathrm{L} \mathrm{DTT}, 0.05 \%$ Triton $\mathrm{X} 100)$. Kinase reactions were stopped by boiling in loading buffer with $\beta$ mercaptoethanol. Samples were run on $8 \%$ SDS-PAGE gels, gels were fixed in $2.5 \%$ glutaraldehyde, and incubated for $1 \mathrm{hr}$. at $55{ }^{\circ} \mathrm{C}$ with $1 \mathrm{~mol} / \mathrm{L} \mathrm{KOH}$ to hydrolyze serine/threonine phosphorylations (to visualize only tyrosine phosphorylations), after which gels were dried and exposed to an X-ray film at $-80^{\circ} \mathrm{C}$.

RNA isolation and quantitative real-time PCR

After overnight starvation, cells were stimulated for 8 or 20 hrs with $0,0.5,5$, or 50 $\mathrm{ng} / \mathrm{ml}$ VEGF. Cells were lysed and total RNA was isolated using the RNeasy mini kit (Qiagen) and DNAse treatment according to manufacturer's instructions. cDNA was 
synthesized from 10 ng RNA using random hexamer primers and oligoDT primers (Fermentas) and the Omniscript RT polymerase kit (Qiagen). Quantitative real timePCR was performed on a MyiQ system (Bio-Rad) using Platinum SYBR Green qPCR Supermix UDG (Invitrogen) containing 1:50 Fluorescein (Molecular Probes) and the following primers with annealing temperatures of $60{ }^{\circ} \mathrm{C}$ : human ADAM-10: $\mathrm{FW}$ 5'TTGCCTCCTCCTAAACCACTTCCA-3' and Rev 5'-AGGCAGTAGGAAGAACCAAGGCAA3'; human ADAM-17: Fw primer 5'-GGGAAGTGACTTAGCAGATG-3' and Rev. 5'CTAGATTCACCTTCACCTTACC-3'; human $\beta$-actin: $\mathrm{FW}$ primer $5^{\prime}$ ATCCTCACCCTGAAGTACCC-3' and Rev 5'-CACGCAGCTCATTGTAGAAG-3'; human GAPDH: FW 5'-GCCTCAAGATCATCAGCAAT-3' and Rev 5'-GGA CTGTGGTCATGAGTCCT-3'; porcine $\beta$-actin: Fw 5'- GCATCCTGACCCTCAAGTAC - 3 ' and Rev 5'-CACGCAGCTCGTTGTAGAAG-3'; porcine GAPDH: Fw 5'-GTGTCGGTT GTGGATCTGA-3' and Rev 5'-CCTGCTTCACCACCTTCTT-3' (Eurogentec). Data were analyzed with the iQ5 software (Bio-Rad) and expressed relative to the housekeeping gene expression using the ddCt- method.

\section{VEGFR-2 ectodomain shedding}

PAEC-KDR were cultured in T25 cell culture flasks and serum-starved overnight once 70-90\% confluency was reached. The next day, cells were given $2 \mathrm{ml}$ fresh

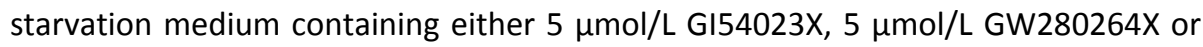
DMSO as control and stimulated for $5 \mathrm{hrs}$ with 0 or $50 \mathrm{ng} / \mathrm{ml}$ VEGF. Cell culture medium was collected and concentrated $40 \mathrm{x}$ by $10 \mathrm{~min}$ centrifugation at $3000 \mathrm{~g}$ using Vivaspin2 columns with 10000 MWCO PES filter (Sartorius). Complete concentrated media were loaded on $8 \%$ SDS-PAGE gels and release of soluble VEGFR-2 ectodomain was detected by Western blotting.

\section{Western Blotting}

ECs were lysed by cell scraping and rotating for $30 \mathrm{~min}$ at $4^{\circ} \mathrm{C}$ in a $1 \% \mathrm{NP} 40$ lysisbuffer containing $1 x$ protease inhibitor cocktail (Roche) and $100 \mu \mathrm{mol} / \mathrm{L} \mathrm{NaVO}_{3}$. Equal amounts of proteins were separated on $8 \%$ SDS-PAGE gels and transferred to a nitrocellulose membrane (Amersham). Blots were blocked with $5 \%$ milk in PBS$0.1 \%$ Tween, and incubated with 1:5000 rabbit anti-human ADAM-10 antibody (Chemicon), 1:500 mouse anti-human VEGFR-2 antibody (Acris) or 1:5000 mouse monoclonal anti-human VE-cadherin antibody (Santa Cruz) in blocking solution, followed by addition of the appropriate horseradish peroxidase labelled secondary antibody and West Dura ECL detection (Pierce). As loading control, $\beta$-actin expression was detected using 1:15000 mouse anti- $\beta$-actin monoclonal antibody (Sigma). Chemiluminescence was detected using LAS 1000 imaging system (Fuji), analyzed using Aida software analysis and results were expressed relative to $\beta$-actin expression. 
Electric Cell-substrate Impedance Sensing (ECIS)

HUVEC were grown to confluency on fibronectin-coated electrode arrays. When basal electrical resistance of the monolayer reached a plateau, cells were pre-

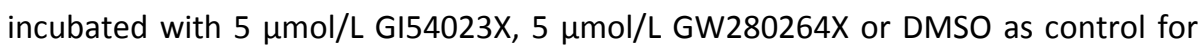
$30 \mathrm{~min}$. After steady state was reached, cells were treated with 0 or $100 \mathrm{ng} / \mathrm{ml} \mathrm{VEGF}$ and electrical resistance was monitored on-line with the ECIS-Model-100 Controller (BioPhysics, Troy, USA) and changes in resistance were analyzed as previously described ${ }^{3}$.

At steady state conditions, an elevated field ( $3 \mathrm{~V}$ at $40,000 \mathrm{~Hz}$ for $10 \mathrm{~s}$ ) was applied to a confluent layer of HUVEC to wound the cells on the electrode and wound healing was monitored for $10-20 \mathrm{~h}$ by ECIS measuring transendothelial electrical resistance.

\section{Cell migration}

Monocytes were isolated from human peripheral blood by negative selection using the MACS monocyte isolation kit II (Miltenyi Biotech) as described previously ${ }^{4}$. ECs were serum starved overnight and migration was assayed using a modified Boyden chamber $^{5}$. A total of 25000 cells treated with $0-5 \mu \mathrm{mol} / \mathrm{L}$ GI54023X (selective ADAM-10 inhibitor) were loaded in quadruplicate onto a 5-8 $\mu \mathrm{m}$ pore membrane (Whatmann) uncoated for monocytes or coated with $5 \mu \mathrm{g} / \mathrm{ml}$ fibronectin (Sigma) for EC, respectively. Migration was stimulated with $10 \mathrm{ng} / \mathrm{ml} \mathrm{VEGF}$ or $10-^{8} \mathrm{~mol} / \mathrm{L} \mathrm{fMLP}$ (N-formyl-Met-Leu-Phe, Sigma). After 1.5 hrs (monocytes) or 4 hrs (EC) migration, membranes were fixed with $100 \%$ ethanol and stained with Giemsa (Merck). Nonmigrated cells on the upper membrane surface were removed before embedding the membrane in Neomount (Merck). Migrated cells were counted in five random high power fields per well. 


\section{Figures}
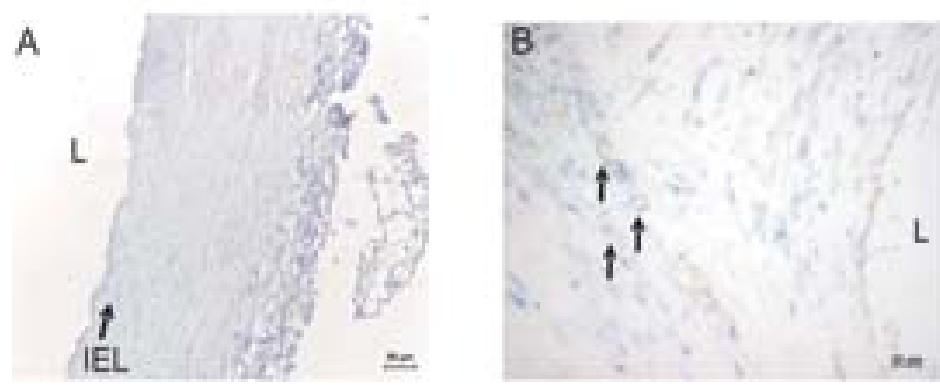

Supplemental figure I .Immunohistochemical staining of ADAM-10 in human mammary artery (A) and double-staining of ADAM-10 (blue) and CD31 (brown) in advanced atherosclerotic plaque (B). L=lumen, IEL=internal eleastic lamina.

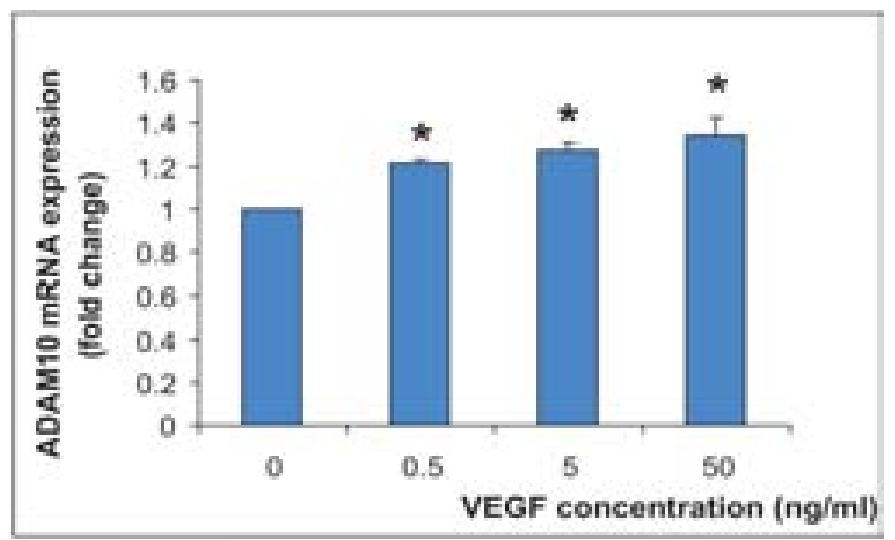

Supplemental figure II. VEGF induces ADAM-10 mRNA expression after $8 \mathrm{hrs}$ stimulation in HUVECs as assessed by quantitative real-time PCR and expressed relative to $\beta$-actin and GAPDH housekeeping gene expression. ${ }^{*} \mathrm{p}<0.05, \mathrm{n}=3$. 


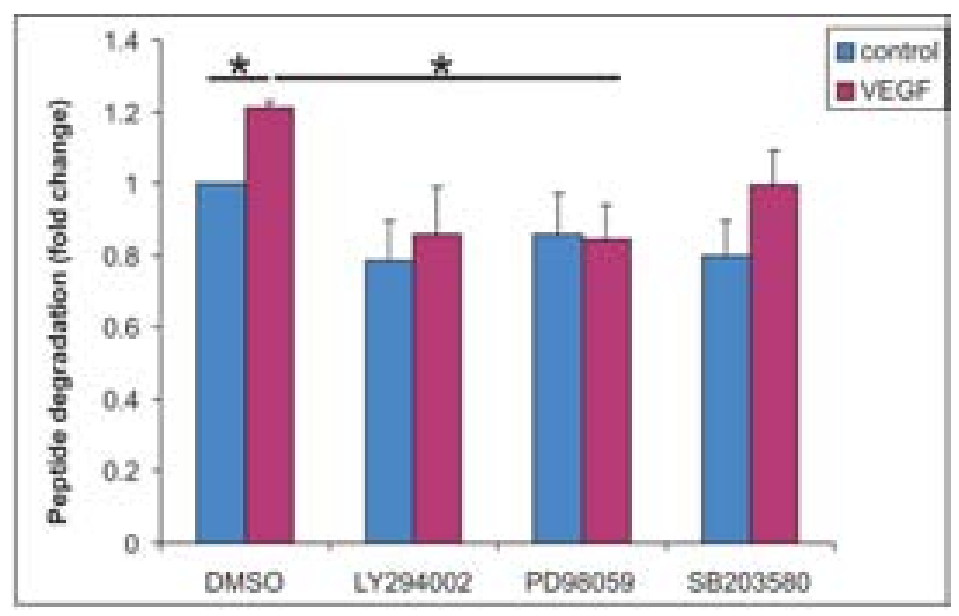

Supplemental figure III. Using $10 \mu \mathrm{mol} / \mathrm{L}$ of the specific inhibitors LY294002 (PI3K), PD98059 (ERK1/2) and SB203580 (p38) we showed VEGF-induced fluorogenic peptide degradation was significantly reduced by inhibition of ERK1/2 $\left({ }^{*} p=0.02, n=3\right)$ and showed similar results for PI3K inhibition $(p=0.06$, $n=3)$.
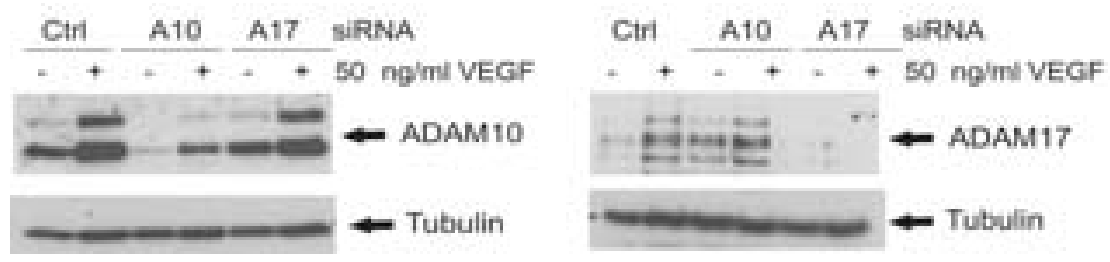

Supplemental figure IV. ADAM-10 Western blot (left) and ADAM-17 Western blot (B) detection shows reduction of ADAM-10 expression by ADAM-10 siRNA transfection, but not ADAM-17 or control siRNA, while ADAM-17 expression is specifically reduced by ADAM-17 siRNA transfection in HUVEC $(n=3)$. 
Table I. Results of Yeast-two-hybrid screening using the intracellular domain of human VEGFR-2 as bait and screening against a human placenta cDNA library.

\begin{tabular}{|c|c|c|c|}
\hline Screen nr. & Number of transfections & Number of colonies & Number of $\beta$-gal \\
\hline 1 & $1.64 \times 10^{4}$ & 0 & 0 \\
\hline 2 & 120 & 2 & 2 \\
\hline 3 & 3361 & 24 & 4 \\
\hline 4 & $3.4 \times 10^{4}$ & 401 & 40 \\
\hline 5 & $2.36 \times 10^{4}$ & 563 & 47 \\
\hline total & & 990 & 93 \\
\hline
\end{tabular}

Table II. Semi-quantitative analysis of ADAM-10 expression in human atherosclerotic plaques. Staining scores: 0 = negative, $1=$ faint, $2=$ moderate, $3=$ strong. ${ }^{*} p<0.05$ vs early and advanced stable.

\begin{tabular}{|c|c|c|c|c|}
\hline Plaque number & $\begin{array}{l}\text { Normal vessel } \\
\text { wall }(n=2)\end{array}$ & Early $(n=5)$ & $\begin{array}{l}\text { Advanced stable } \\
(n=8)\end{array}$ & Ruptured ( $n=5)$ \\
\hline 1 & 0 & 1 & 2 & 2 \\
\hline 2 & 0 & 2 & 2 & 3 \\
\hline 3 & & 2 & 3 & 3 \\
\hline 4 & & 1 & 2 & 3 \\
\hline 5 & & 2 & 3 & 3 \\
\hline 6 & & & 2 & \\
\hline 7 & & & 1 & \\
\hline 8 & & & 2 & \\
\hline Mean \pm SEM & $0 \pm 0$ & $1.6 \pm 0.2$ & $2.1 \pm 0.2$ & $2.8 \pm 0.2^{*}$ \\
\hline
\end{tabular}

Table III. Semi-quantitative analysis of ADAM-10 expression in human colon tissue. Staining scores: $0=$ negative, $1=$ faint, $2=$ moderate, $3=$ strong. ${ }^{*} p<0.05$ vs normal.

\begin{tabular}{|llllll}
\hline & Colon tissue & \multicolumn{3}{cl}{ Microvasculature } \\
$\begin{array}{l}\text { Tissue } \\
\text { number }\end{array}$ & Normal $(n=5)$ & Carcinoma $(n=5)$ & Normal $(n=5)$ & Carcinoma $(n=5)$ & $\begin{array}{l}\text { Tumor } \\
\text { Stroma } \\
(n=5)\end{array}$ \\
1 & 1 & 2 & 1 & 2 & 1 \\
2 & 2 & 2 & 1 & 2 & 2 \\
3 & 1 & 3 & 2 & 3 & 1 \\
4 & 1 & 3 & 1 & 2 & 1 \\
5 & 1 & 3 & 2 & 3 & 2 \\
Mean \pm SEM & $1.2 \pm 0.2$ & $2.6 \pm 0.2^{*}$ & $1.4 \pm 0.2$ & $2.4 \pm 0.2^{*}$ & $1.4 \pm 0.2$
\end{tabular}




\section{References}

1. Waltenberger J, Claesson-Welsh L, Siegbahn A, Shibuya M, Heldin CH. Different signal transduction properties of KDR and Flt1, two receptors for vascular endothelial growth factor. $J$ Biol Chem. 1994;269:26988-26995.

2. van der Schaft DW, Seftor RE, Seftor EA, Hess AR, Gruman LM, Kirschmann DA, Yokoyama Y, Griffioen AW, Hendrix MJ. Effects of angiogenesis inhibitors on vascular network formation by human endothelial and melanoma cells. J Nat/ Cancer Inst. 2004;96:1473-1477.

3. van Buul JD, van Alphen FP, Hordijk PL. The presence of alpha-catenin in the VE-cadherin complex is required for efficient transendothelial migration of leukocytes. Int J Biol Sci. 2009;5:695-705.

4. Stadler N, Eggermann J, Voo S, Kranz A, Waltenberger J. Smoking-induced monocyte dysfunction is reversed by vitamin C supplementation in vivo. Arterioscler Thromb Vasc Biol. 2007;27:120-126.

5. Waltenberger J, Lange J, Kranz A. Vascular endothelial growth factor-A-induced chemotaxis of monocytes is attenuated in patients with diabetes mellitus: A potential predictor for the individual capacity to develop collaterals. Circulation. 2000;102:185-190. 



\section{Chapter 4}

\section{Developmental Coronary Maturation is Disturbed by Aberrant Cardiac VEGF- expression and Notch-Signaling}

Nynke M.S. van den Akker ${ }^{1}$, Vincenza Caolo ${ }^{2}$, Lambertus J. Wisse ${ }^{1}$, Patricia P.W.M. Peters $^{2}$, Robert E. Poelmann ${ }^{1}$, Peter Carmeliet ${ }^{3,4}$, Adriana C. Gittenberger-de Groot $^{1^{*}}$, and Daniel G.M. Molin ${ }^{1,2^{*}}$

${ }^{1}$ Department of Anatomy and Embryology, Leiden University Medical Center, Einthovenweg 20, PO Box 9600, 2300 RC Leiden, The Netherlands; ${ }^{2}$ Department of Physiology, CARIM, Maastricht University, Maastricht, The Netherlands; ${ }^{3}$ Center forTransgene Technology and Gene Therapy, K.U. Leuven, Leuven B-3000, Belgium; and ${ }^{4}$ Department of Transgene Technology and Gene Therapy, VIB, Leuven B-3000, Belgium

* Equal contribution

Modified after Cardiovascular Research, 2008;78:366-75. 



\begin{abstract}
Currently, many potential cardiac revascularization therapies are targeting the Vascular Endothelial Growth Factor (VEGF)-pathway, with variable success. Knowledge regarding the role of the VEGF/Notch/ephrinB2-cascade in (ab)normal coronary development will provide information on the subtle balance of VEGFsignaling in coronary maturation and might enhance our therapeutic possibilities. The effect of VEGF-isoforms on coronary development was explored in vivo using immunohistochemistry and RT-qPCR on Vegf120/120 mouse embryos, solely expressing VEGF120. In vitro, human arterial coronary endothelial cells were treated with VEGF121 or VEGF165 upon which RT-qPCR was performed. In vivo, mutant coronary arterial endothelium showed a decrease in protein expression of arterial markers such as cleaved Notch1, Delta-like4 and ephrinB2 concomitant with an increase of venous markers such as chicken ovalbumin upstream promoter transcription factor II. The venous endothelium showed the opposite effect, which was confirmed on mRNA-level. In vitro, mRNA-expression of arterial markers highly depended on the VEGF-isoform used, with VEGF165 having the strongest effect. Also, coronary arteriogenesis was anomalous in the mouse embryos with decreased arterial and increased venous medial development as shown by staining for smooth muscle $\alpha$-actin, Delta-like1 and Notch3. We demonstrate that VEGF-isoform related spatiotemporal cardiac alterations in the VEGF/Notch/ephrinB2-cascade lead to disturbed coronary development. This knowledge can contribute to optimizing therapies targeting VEGF-signaling by enabling balancing between angiogenesis and vascular maturation.
\end{abstract}




\section{Introduction}

Morphological diversity between different populations of endothelial cells (ECs) has long been recognized to relate to functional variation and as such proper physiology. More recently, differential expression profiles between arterial, microcirculatory, venous and lymphatic endothelial populations have been described $^{1}$, further underscoring endothelial diversity. Both intrinsic and microenvironmental differences are instructive for these endothelial characteristics $^{2}$. Abnormalities can lead to endothelial dysfunction and subsequent pathogenesis, as found for maternal diabetes-related fetal endothelial dysfunction ${ }^{3}$, increased nuchal translucency ${ }^{4}$, tumor angiogenesis ${ }^{5}$ but also for coronary atherosclerosis ${ }^{6}$ and adult venous bypass graft disease ${ }^{7}$.

Numerous factors associate with endothelial functioning in processes such as vasculogenesis, angiogenesis and arteriogenesis, with one of the key factors being Vascular Endothelial Growth Factor (VEGF) ${ }^{8}$. Therefore, it is not surprising that many potential therapies for ischemic cardiovascular disease target VEGF, although clinical success is less than expected ${ }^{8}$. VEGF exerts its main effects through binding to the VEGF receptor VEGFR- ${ }^{8}$. Its coreceptor neuropilin-1 (NP-1) can enhance this effect ${ }^{9}$. VEGF-signaling can upregulate members of the Delta-like/Jagged/Notchfamily, proteins highly important in cardiovascular development ${ }^{10,11}$. Several members of this family are specifically expressed by arterial ECs, including Notch1, Notch4 and Delta-like4 (DII4) $)^{12}$. Upon stimulation of the Notch-receptor, its cytoplasmic domain becomes cleaved and translocated to the nucleus. There, it forms a complex with other nuclear proteins, which upregulates transcription of Notch-signaling specific transcription factors (i.e. Hes, Hey). These can enhance arterial EC-specific ephrinB2-expression ${ }^{10}$, suggesting a role for the VEGF/Notch/ephrinB2-cascade in arterial differentiation ${ }^{11}$.

The development of the coronary vasculature is of eminent importance for the final phases of cardiogenesis. Disbalance in these processes might lead to a dysfunctional vascular bed which will predispose for coronary diseases. Coronary development starts with the occurrence of a subepicardially located primitive endothelial network. This becomes connected to the systemic circulation downstream to the right atrium and upstream to the ascending aorta through coronary orifices at the level of the left and right sinus of the semilunar valves ${ }^{13}$. After connection to the circulation is established, arterial differentiation of the most proximal ECs becomes obvious and, subsequently, arteriogenesis is stimulated by inducing vascular smooth muscle cell (vSMC) migration and differentiation ${ }^{13,14}$. In the coronary system, vSMCs are mainly recruited from the population of epicardium-derived cells (EPDCs), which arises through epithelial-to-mesenchymal transformation of cells of the epicardium ${ }^{15}$. In addition, microcirculatory and venous endothelial differentiation and remodeling of the primitive endothelial network into a fully functional coronary system will take place. 
We recently described the Vegf120/120 mouse model that solely expresses the non-heparin binding isoform VEGF120. Structural cardiac abnormalities such as Tetralogy of Fallot (TOF) were found concomitant with alterations in VEGF and Notch-signaling ${ }^{16,17}$. This mouse model presents postnatally with impaired myocardial angiogenesis ${ }^{18}$ and altered retinal arterio-venous differentiation ${ }^{19}$. In this paper, we show that changes in spatiotemporal distribution of VEGF and Notchsignaling -due to altered VEGF-isoform expression in Vegf120/120 embryos- lead to anomalous coronary EC differentiation and disturbed arteriogenesis.

\section{Methods}

\section{Mouse Experiments}

All animal experiments were approved by the Animal Ethics Committee of the Leiden University and executed according to the Guide for the Care and Use of Laboratory Animals published by the US National Institute of Health (NIH Publication No. 85-23, revised 1996). For an extensive description of mouse experiments see $^{17}$.

\section{Immunohistochemistry, in situ hybridisation and microscopy}

Immunohistochemistry was performed as described ${ }^{17}$. In short, immunohistochemistry was carried out on sections of $5 \mu \mathrm{m}$ of 4\%PFA/0.01 Mol/L phosphate buffered fixed, paraffin embedded tissue. Antigen retrieval was achieved either by heating the sections ( 12 minutes to $98^{\circ} \mathrm{C}$ ) in citric acid buffer $(0.01 \mathrm{Mol} / \mathrm{L}$, $\mathrm{pH6.0)}$ or by incubating them for $6 \mathrm{~min}$ in $0.1 \mathrm{mg} / \mathrm{mL}$ Pronase $\mathrm{E}$ (1.07433.0001, Merck, Darmstadt, Germany) in PBS. Visualization was performed with the DABprocedure and Mayer's hematoxilin was used as a counterstaining. Differences between genotypes were scored per immunostaining in at least 3 different embryos per genotype per age-group (i.e. E10.5-E14.5 or E15.5-E19.5) that were processed within one and the same experiment. The primary antibodies were checked for false positive signals. An extensive description of the primary antibodies used is listed in Table S1. The $\alpha$-Notch4-antibodies listed in Table S1 were tested to bind aspecific to the material used and therefore no characteristics were mentioned. For in situ hybridisation, sense and anti-sense ${ }^{35}$ S-radiolabelled Vegf- $A$ RNA probes were transcribed using a 451-bp clone encoding for the mouse Vegf-120 isoform (pVEGF2; kindly provided by Dr. G. Breier, University of Technology, Dresden, Germany). Micrographs were made using an Olympus AX70-microscope fitted with Olympus UPlanApo-objectives (Olympus, Tokyo, Japan). The camera used was an Olympus DP12 and micrographs were fitted into a panel using Adobe Photoshop CS2 (Adobe, San Jose, USA). 


\section{D-reconstructions}

3D-reconstructions were performed as described ${ }^{4}$. In short, micrographs were made of E18.5 wild type and Vegf120/120 hearts and converted to 3D using ResolveRT ${ }^{\mathrm{TM}}$ software (Template Graphics Software Inc., San Diego, USA).

\section{Morphometry}

For morphometry, VEGFR-2 stained sections of 8 embryos (4 Vegft/+ and 4 Vegf120/120; E17.5-E19.5) were used. To calculate the total heart volume and the ratio myocardial to vascular volume, we performed volume measurements for total heart volume, myocardium and coronary veins/microvasculature using the Cavalieri method $^{20}$. Statistical analysis was performed using a Mann-Whitney test with SPSS 11.0 (SPSS Inc., Chicago, USA) software.

\section{Cell culture}

Human coronary artery endothelial cells (HCAECs) were obtained from Cambrex (Verviers, Belgium) and cultured according to the protocol of the manufacturer. Cells at passage 9 were seeded at a density of 3500 cells $/ \mathrm{cm}^{2}$ and cultured to a $70 \%$ subconfluent monolayer in EGM-2-MV BulletKit without bFGF and VEGF165 supplemented with 5\% Fetal Bovine Serum (Cambrex). Subsequently, HCAECs were stimulated for 24 hours with $100 \mathrm{ng} / \mathrm{mL}$ recombinant human VEGF165 or VEGF121 (RELIATech $\mathrm{GmbH}$, Braunschweig, Germany). Cells were harvested according to the manufacturer's protocol using a trypsin/EDTA and a Trypsin neutralization solution (Cambrex). Cell number was determined using trypan blue dye and the Neubauer improved chamber method. mRNA was isolated as described below. Per sample, $100,000-140,000$ cells were harvested. Samples stimulated with either VEGF165 or VEGF121 provided higher cell numbers than unstimulated samples (cultured without VEGF).

\section{$R T-q P C R$}

Total RNA from triplicate HCAEC cultures or from cardiac tissue of E18.5 wild type and Vegf120/120 embryos ( $n=3$ per group) was isolated by using the RNeasy microkit Qiagen with DNAse treatment (Qiagen, $\mathrm{GmbH}$, Hilden, Germany). Sample with sufficient RNA quality (OD260/280>1.9, RIN>8.0) and content were approved for analysis. A total of $100 \mathrm{ng}$ total RNA per sample was subjected to reverse transcription (RT). qPCR was performed by using Superscript ${ }^{\mathrm{TM}} \mathrm{III}_{\text {Platinum Two-step }}$ qRT-PCR kit with SYBR green (Invitrogen, Paisley, UK) and primer concentration of $10 \mu \mathrm{M}$. qPCR reactions were run on a MyiQ Single-Color Real time PCR detection System (Bio-Rad, Veenendaal, the Netherlands). Primers were designed with oligoperfect $^{\mathrm{TM}}$ Designer (Invitrogen), Primer3 and Mfold (http: //www.idtdna.com/scitools/Application/mfold/) and were synthesized by Eurogentec (Seraing, Belgium). All primers used met the requested qPCR efficiency 
(between $80-105 \%$ ) and were found to be gene-specific. Primer sequences are found in Table S2. Data analysis was performed using a Microsoft Excel spreadsheet based on the qBase program ${ }^{21}$. Statistic significance was determined by applying one-way ANOVA/Tukey (SigmaStat v2.03, Systat Software, San Jose, USA) testing. Sample with a probability value $(p)<0.05$ were regarded to be significant different between groups. HCAEC samples were normalized for input based on both $\beta$-actin and GADPH-values. For the E18.5 embryos normalization was based on the panendothelial marker Tie2. No significant differences were found for Tie2 between wild type and Vegf120/120 littermates as determined after normalization for BActin and Gapdh expression (correction for cDNA input). Using immunohistochemistry, no altered myocardial expression of the genes investigated was found. Myocardial Notch4-expression was not determined due to aspecifc binding of the antibodies tested (see table S1), however, Notch4 has been reported to be highly selective for ECs during development ${ }^{22}$. 


\section{Results}

Anomalous coronary morphology in Vegf120/120 mouse embryos

In line with literature on murine coronary development ${ }^{23}$, the first appearance of coronary ECs and vessels was seen at E11.5 when using an $\alpha$-VEGFR-2-staining for visualization of the ECs. We observed that at this stage, primitive coronaries already connected (in)directly to the right atrium in all embryos, suggesting normalarly coronary development in mutant embryos (Figures $1 \mathrm{~A}-\mathrm{B}, \mathrm{E}, \mathrm{H})$. However, abnormally large coronary veins were discerned in 22/33 early-stage (E11.5-E13.5) mutant cases (Figure $1 \mathrm{~A}-\mathrm{B}, \mathrm{F}-\mathrm{G}$ ).

In wild type embryos, two definitive coronary orifices, originating from the left and right aortic sinus respectively, were observed in 24/24 cases of E15.5 and older. In contrast, only $15 / 28$ Vegf120/120 embryos of these stages presented with 2 coronary orifices, whereas others showed only one $(2 / 28)$, three $(7 / 28)$ or in one case four orifices (1/28 and Table 1$)$. After formation of the coronary orifices, maturation of coronary arteries was obvious in normal embryos. In 22/28 mutant embryos between E15.5 and E19.5, arteriogenesis was severely impaired (Table 1; Figure 1C-D and movies online). Half of the mutant embryos showed abnormalities in anatomy of the primary coronary branches (Table 1; Figure 1C-D and movies online), with the main artery supplying the interventricular septum (IVS) either having its own coronary orifice and/or originating from the left instead of the right sinus, which is normal ${ }^{24}$. All embryos in which the IVS-branch originated (in)directly from the left sinus were previously diagnosed with TOF $(5 / 28)^{17}$. For a more detailed description of the abnormalities in coronary branching patterns we refer to the online supplement.

In 50\% of E15.5-E19.5 Vegf120/120 embryos (14/28) enlarged coronary veins (Table 1; Figure $1 \mathrm{~J}-\mathrm{K})$, already evident at earlier stages, and enlarged cardiac lymphatics (16/28; Table 1; Figure 1L-M), visualized using staining for LYVE-1 of which expression is specific for lymphatic ECs, were encountered. Also, in late stage embryos, ventriculo-coronary arterial communications (VCACs) and/or coronary arterio-venous shunts were evident (Table 1; Figure 1N,O). Arterio-venous shunts between the aortic arch and vena cava superior were found in 3 cases (all E19.5; Figure 1P). 
Table 1. Coronary abnormalities in Vegf120/120 embryos of E15.5-E19.5.

\begin{tabular}{lll}
\hline Coronary Anomaly & No/Total & $\%$ \\
\hline Abnormal number of coronary orifices & $10 / 28$ & 36 \\
$\quad<2$ & $2 / 28$ & 7 \\
$\quad>2$ & $8 / 28$ & 29 \\
Small coronary arteries & $22 / 28$ & 79 \\
Abnormal origin IVS-branch & $14 / 28$ & 50 \\
Large coronary veins & $14 / 28$ & 50 \\
& & \\
Large cardiac lymphatics & $16 / 28$ & 57 \\
Increased microvascular density & $17 / 28$ & 61 \\
VCAC & & \\
\end{tabular}

IVS = indicates interventricular septum; VCAC = indicates ventriculo-coronary artery communication

Spatiotemporal distribution of Vegf in normal and mutant embryonic hearts During early cardiac development (E10.5-E14.5), the distribution of Vegf-expression has been described before ${ }^{17}$ and is likely mainly related to myocardial and to a lesser extent to coronary development. In later stages (E16.5 and older) the predominant area of Vegf-expression was the borderline of compact to trabecular myocardium, overlapping with the region where prominent coronary angiogenesis and arteriogenesis takes place at this stage ${ }^{25}$. Vegf levels in this area were increased in Vegf120/120 embryos (Figure 1Q-R). 

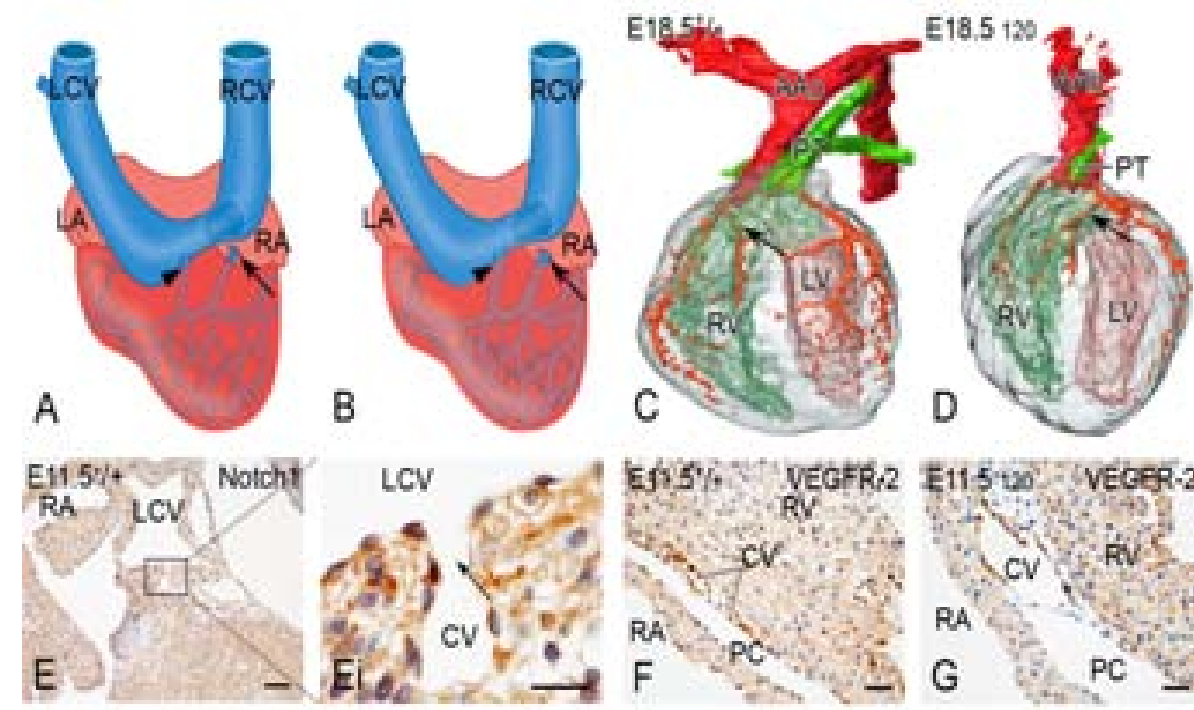

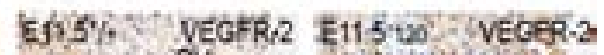
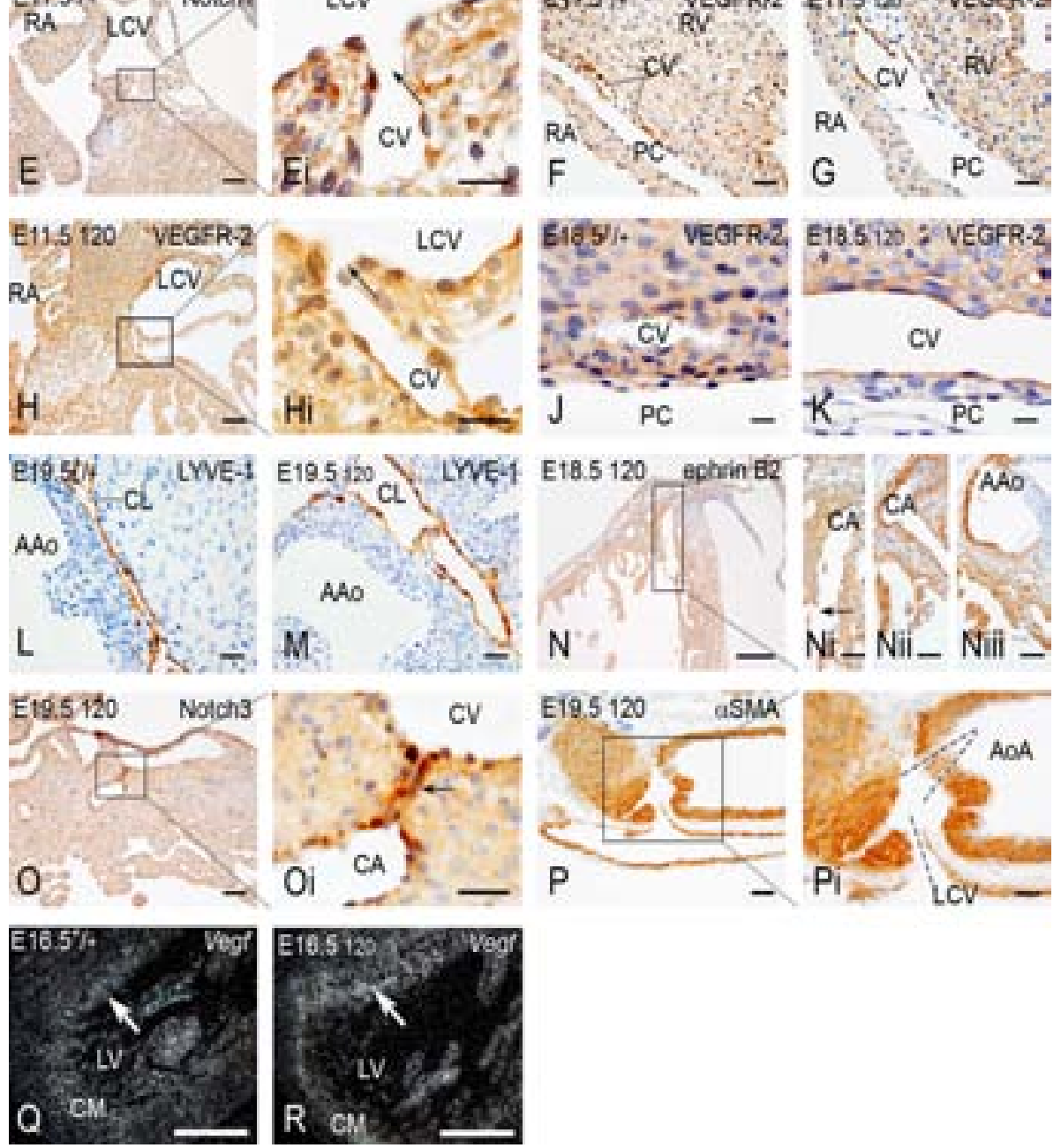
Loss of coronary venous endothelial differentiation in Vegf120/120 embryos

Besides morphological differences in mutant veins, abnormal differentiation of coronary venous ECs was clearly distinguishable from E15.5 onwards (Table S3). High expression per EC of VEGFR-2 was still seen at later stages of development (Figure $1 \mathrm{~J}-\mathrm{K})$, together with anomalous expression of arterial markers including cleaved (nuclear/activated) Notch1, Notch2, Dll4 and ephrinB2 (Figure 2A-B,E-F,I$J, M-N)$, suggesting increased VEGF and Notch-signaling per EC. Expression of the venous EC-markers chicken ovalbumin upstream promoter transcription factor II (COUP-TFII) and EphB4 (Figure $2 \mathrm{Q}-\mathrm{R}, \mathrm{U}-\mathrm{V}$ ) was clearly reduced in mutant coronary venous ECs.

RT-qPCR, normalized for endothelial-specific Tie2-expression, was performed on whole hearts of either wild type mouse embryos or Vegf120/120 littermates of E18.5. A significant increase of Notch1, Notch4 and Hes1 mRNA-levels could be found (Figure 3A). Furthermore, a significant decrease in Ephb4 mRNA was obvious, while a positive trend was noticed for expression of D/l4 and Hey1 (Figure 3A).

$\leftarrow$ Figure 1. Abnormal coronary and aortic arch morphology and Vegf-distribution in Vegf120/120 embryos. Wild type $(+/+)$ and mutants (120) are compared (upper left corner, age in embryonic days (E)) with the staining indicated upper right. At E11.5, a direct (arrow) and an indirect via the left cardinal vein (LCV; arrowhead) connection between the coronary system and the right atrium (RA) are seen in +/+ and 120 embryos (schematic drawings in $A$ and $B$, respectively). The indirect connection is also shown in $E$ $(+/+)$ and $\mathrm{H}(120)$ and arrows in $\mathrm{Ei}$ and $\mathrm{Hi}$. The stainings indicated in $\mathrm{E}$ and $\mathrm{H}$ do not correspond, as differentially stained sections had to be chosen due to the fact that the connection can only be seen in a very small area of the embryos. Mutant coronary arteries are smaller and reveal abnormal morphology of the main branches (arrows in C-D), especially in embryos with TOF (D). Both the aortic arch (AoA) and coronary arteries are shown in red, the pulmonary trunk (PT) in green. At E11.5, an increase in size of the coronary veins $(\mathrm{CV})$ is seen in mutant embryos (compare $F$ and $G$ ). This is maintained throughout development (J-K). An increase in size of cardiac lymphatics $(\mathrm{CL})$, as visualized using a staining for the lymphatic EC-specific marker LYVE-1, is obvious (L-M). In mutant embryos, ventriculo-coronary artery connections (VCAC), visualized using an ephrinB2-staining ( $\mathrm{N}$-Niii; with Nii being a consecutive section of $\mathrm{Ni}$ stained for $\alpha$-SMA and Niii a section $35 \mu \mathrm{m}$ caudal from Nii) and arterio-venous shunts in both the coronary system visualized using a Notch3-staining ( $O$ and arrow in $\mathrm{Oi}$ ) and the AoA ( $P$ and dotted lines in $\mathrm{Pi}$ ) were seen. Locally increased levels of Vegf-mRNA are observed in mutant embryos (arrows in $\mathrm{Q}-\mathrm{R}$ ). Abbreviations: $\mathrm{AAo}=$ ascending aorta; $\mathrm{CA}$ = coronary artery; $\mathrm{CM}=$ compact myocardium; $\mathrm{LA}$ = left atrium; $\mathrm{LV}=$ left ventricle; $\mathrm{PC}=$ pericardial cavity; $\mathrm{RCV}=$ right cardinal vein; $\mathrm{RV}=$ right ventricle. $\mathrm{Scale}$ bar $=60 \mu \mathrm{m}$ (E,H.Ni-P); $20 \mu \mathrm{m}$ (Ei,Hi,Oi); $30 \mu \mathrm{m}$ (F-G,J-K,L-M); $200 \mu \mathrm{m}$ (N,Pi,Q-R). 


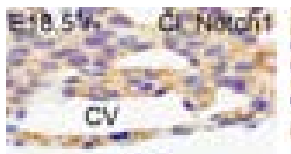

A
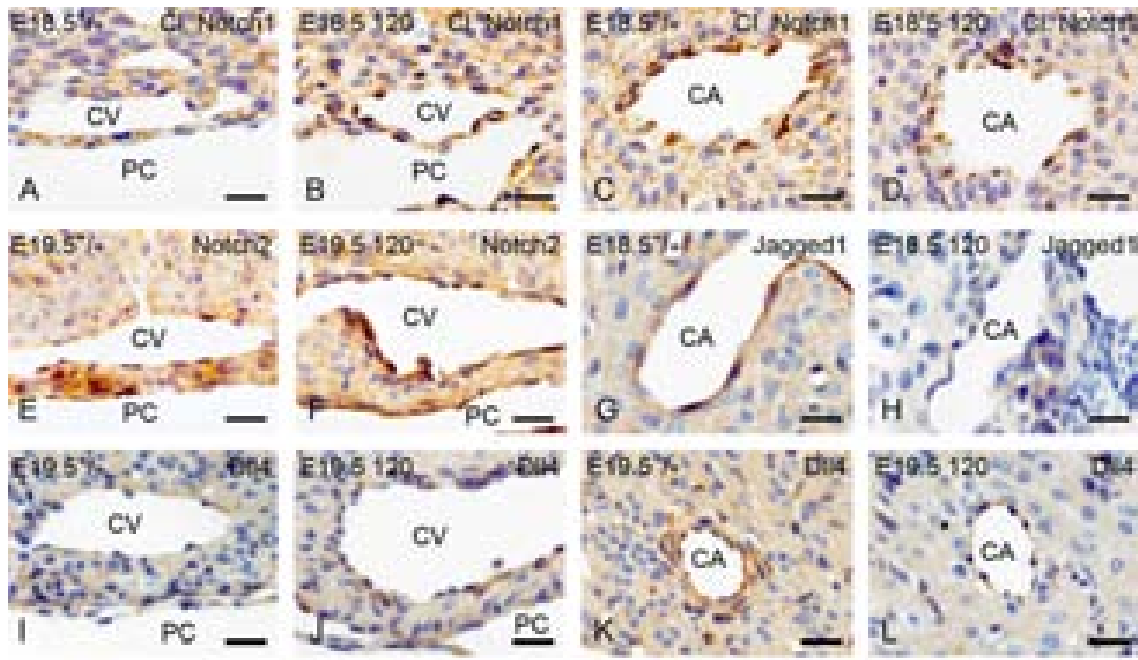

E105\%: ephrin Ba
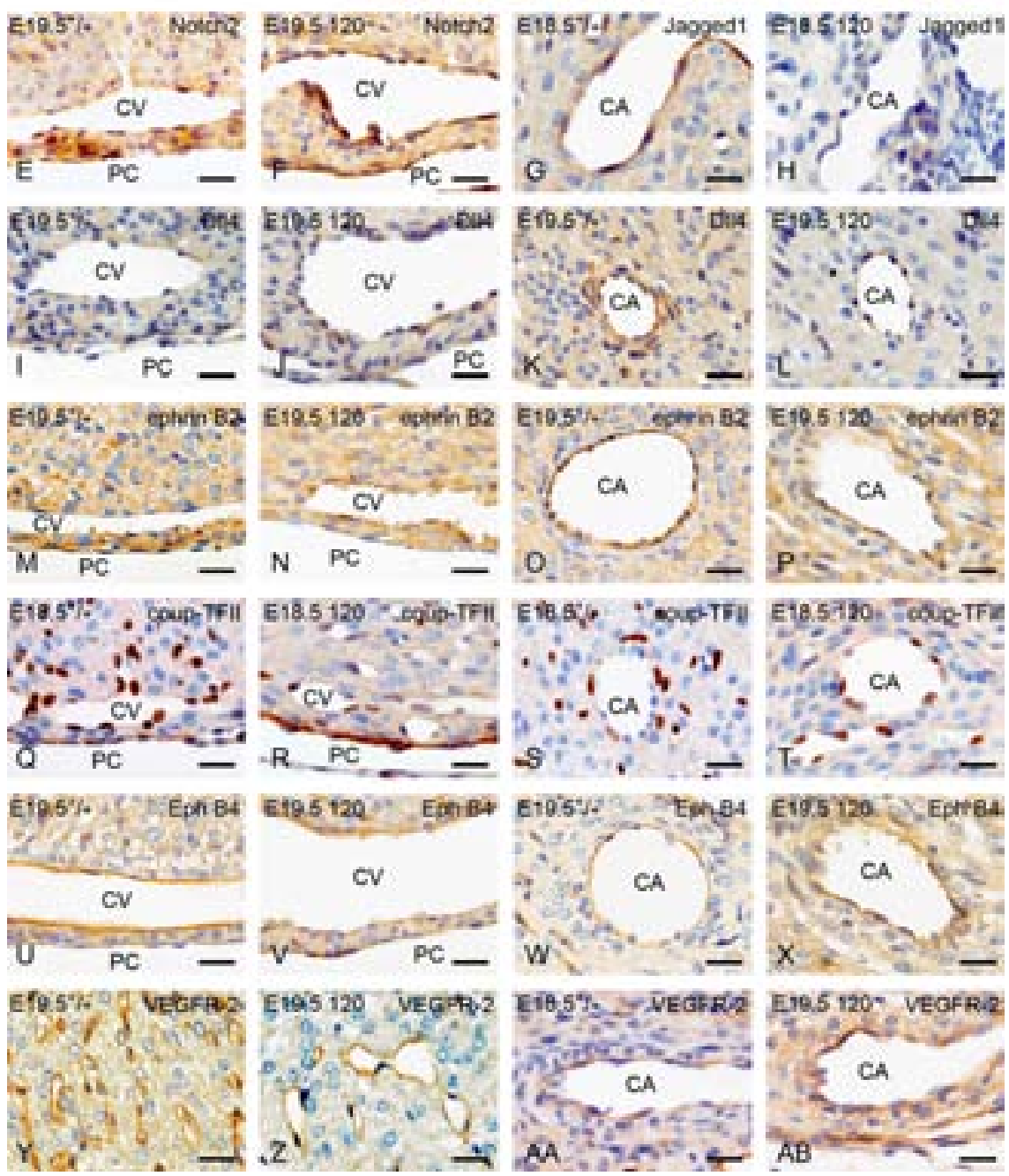

Figure 2. Abnormal EC-differentiation and microvascular morphology in Vegf120/120 embryos.

Stainings performed are indicated in the upper right corner and the genotype (+/+ wild type and 120 mutant) and age in embryonic days (E) upper left. ECs of the mutant coronary veins (CV) showed an increase in staining per cell for arterial markers cleaved Notch1 (A-B), Notch2 (E-F), DII4 (I-J) and ephrinB2 (M-N) and a decrease for the venous markers COUP-TFII (Q-R) and EphB4 (U-V). Increased capillary size is seen (Y-Z). ECs of the coronary arteries (CA) show decreased expression of cleaved Notch1 (C-D), Jagged1 (G-H), DII4 (K-L) and ephrinB2 (O-P) concomitant with an increase in COUP-TFII (S-T), EphB4 (W-X) and VEGFR-2-expression (AA-AB). Abbreviations: Cl. Notch1: cleaved Notch1; PC: pericardial cavity. Scale bars $20 \mu \mathrm{m}$. 


\section{Abnormalities in mutant coronary microvasculature}

Not only the coronary veins, but also the capillaries were enlarged and dilated in Vegf120/120 embryos (Figure 2Y-Z). To quantify these differences morphometry was performed on late-stage embryos. Total heart volumes did not differ significantly between groups (data not shown). The ratio myocardial to coronary vascular volume was significantly decreased in mutant mouse embryos with a $\mathrm{p}$ value of 0.021 (Figure 3B). The differentiation of microvascular ECs in Vegf120/120 mouse embryos, however, was indistinguishable from normal.
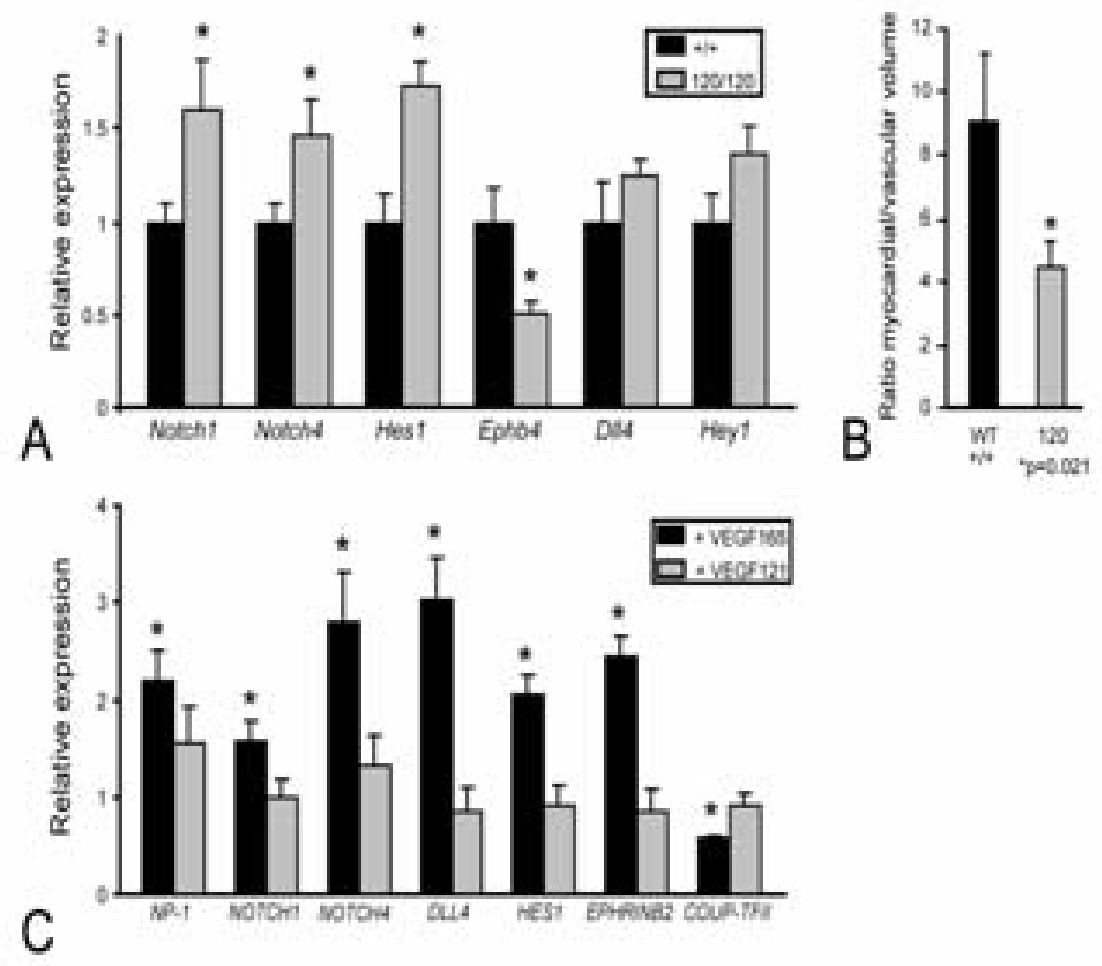

Figure 3. Quantification of alterations in mRNA expression in vivo and in vitro with regard to VEGFisoforms and abnormalities in microvascular density in Vegf120/120 embryos. A significant $\left({ }^{*}\right)$ increase in Notch1, Notch4 and Hes1, a significant decrease in Ephb4 and a trend in increase of DIl4 and Hey1 mRNA expression is seen when performing RT-qPCR normalized for Tie2 on mRNA isolated from wild type $(+/+)$ and mutant (120) hearts of E18.5 (A). Using morphometry, a significant difference $(p=0.021)$ in myocardial/vascular volume is found $(B)$. When human coronary arterial ECs (HCAECs) are cultured with VEGF165 or VEGF121, it is obvious that VEGF165 can while VEGF121 cannot significantly increase expression of arterial markers (NP-1, NOTCH1, NOTCH4, DLL4, HES1 and EPHRINB2) and decrease expression of the venous marker COUP-TFII when compared to HCAECS cultured without VEGF (of which expression is set to 1) (C). 
Coronary arterial endothelial differentiation is impaired in Vegf120/120 embryos

In mutant embryos of E15.5 and older, obvious differences in differentiation of coronary arterial ECs were observed (Table S3). Protein expression levels indicative for arterial maturation, including cleaved Notch1, Jagged1, DII4, and ephrinB2 (Figure 2C-D,G-H,K-L,O-P) were lower per EC, suggesting decreased Notch-signaling. Increased expression per EC of the venous markers COUP-TFII and EphB4 (Figure 2S$\mathrm{T}, \mathrm{W}-\mathrm{X})$ was observed. Due to lack of Notch4-specific antibodies its expression profiles could not be determined (see online information). Although arterial endothelial VEGFR-2-expression normally decreased upon induction of Notchsignaling ${ }^{26}$, this decrease was much less prominent in mutant embryos (Figure 2AA$A B)$.

To determine whether differences in arterial marker expression were VEGF-isoform related, expression of differentiation-specific markers (i.e. arterial; NP-1, NOTCH1, NOTCH4, DLL4, HES1, EPHRINB2 and venous; COUP-TFII) was analyzed in HCAECs in vitro. RT-qPCR on HCAECs cultured with or without VEGF165 or VEGF121 showed that VEGF121 was much less able to affect expression than VEGF165. VEGF165 significantly induced expression levels of arterial markers and decreased expression of COUP-TFII (Figure 3C).

\section{Abnormal arteriogenesis is evident in Vegf120/120 mouse embryos}

Anomalous coronary endothelial differentiation observed in mutant mouse embryos was concomitant with abnormal arteriogenesis. Coronary arteries of mutant embryos of E15.5 and older showed a decrease in medial development with a reduction in number of medial cells and abnormal morphologic and differentiation characteristics as evident by staining for the SMC-marker aSMA (Figure 4A-B). The spatiotemporal protein expression of NP-1, DII1, Jagged2 and Notch3 was analyzed and a decrease in number of cells positive for these markers was seen in the medial layer of the coronary arteries of mutant embryos (Figures $4 \mathrm{E}-\mathrm{F}, \mathrm{I}-\mathrm{J}, \mathrm{M}-\mathrm{N}, \mathrm{Q}-\mathrm{R}$ ). In contrast, the coronary veins of mutant embryos were surrounded by a larger number of $\alpha \mathrm{SMA}$ positive cells when compared to wild type littermates (Figure 4CD). These cells were also positive for the SMC-related markers NP-1, DII1 and Jagged2 (Figures $4 \mathrm{G}-\mathrm{H}, \mathrm{K}-\mathrm{L}, \mathrm{O}-\mathrm{P}$ ). Additionally, the pericyte-coverage of the coronary microvasculature as determined by Notch3-staining showed a marked decrease in number of positive cells in mutant mouse embryos (Figure 4S-T). 

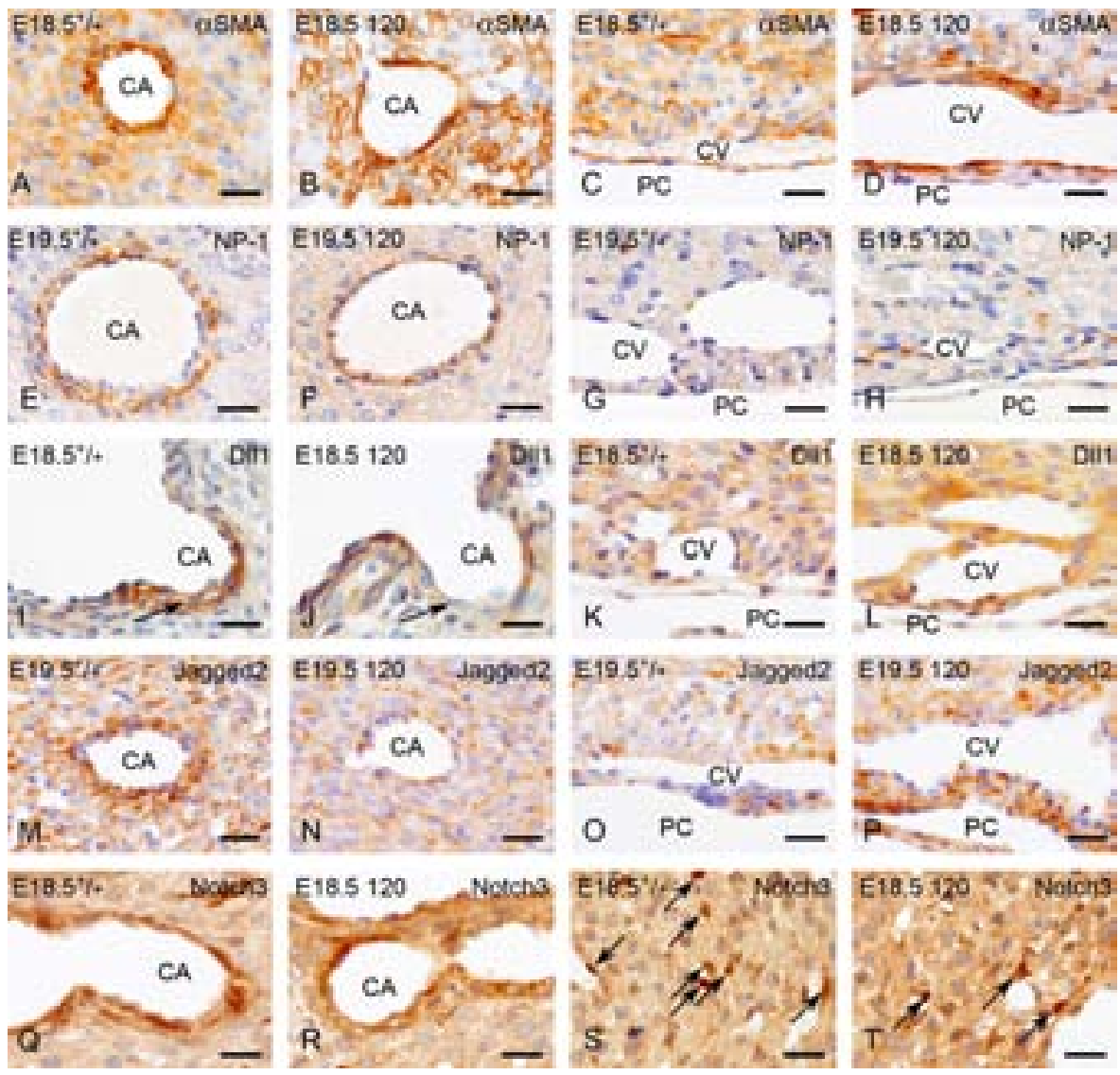

Figure 4. Abnormal coronary arteriogenesis in Vegf120/120 embryos. The expression of several different markers (upper right) in vSMCs/pericytes was compared between wild type $(+/+)$ and mutants (120; upper left together with embryonic age in days $(E))$. A decrease in medial cells expressing $\alpha$ SMA $(A-B), N P-1(E-F)$, Dll1 (I-J), Jagged2 $(M, N)$ and Notch3 (I-J) is obvious in the coronary arteries media (CA) of mutants while an increase of $\alpha \mathrm{SMA}(\mathrm{C}-\mathrm{D}), \mathrm{NP}-1$ (G-H), DII1 (K-L) and Jagged2-positive cells (O-P) surrounds the coronary veins (CV). A decrease in number of Notch3-positive pericytes is seen in mutant coronary microvasculature (K-L). Abbreviation: $\mathrm{PC}=$ pericardial cavity. Scale bars $=20 \mu \mathrm{m}$. 


\section{Discussion}

The loss of the Vegf164 and Vegf188 isoforms in Vegf120/120 mutants results in spatiotemporal alterations in VEGF and Notch-signaling in the heart. These alterations coincide with abnormalities in coronary endothelial differentiation and subsequent arteriogenesis. The data provide us with further insight on the role of both VEGF-isoform and spatiotemporal VEGF-distribution on coronary differentiation and maturation.

\section{Anomalous endothelial differentiation of the Vegf120/120 coronary system}

VEGF-signaling influences vasculogenesis and angiogenesis, but also arterial endothelial differentiation ${ }^{11,27}$. VEGF-signaling in ECs can upregulate expression of members of the Notch-signaling family, which instructs ephrinB2-expression and defines arterial differentiation ${ }^{11,27}$. In venous ECs, expression of these proteins is inhibited by the vein-specific transcription factor COUP-TFII, thereby favoring EphB4-expression and venous differentiation ${ }^{28}$. We show in vitro that addition of VEGF165, but not of VEGF121, leads to significant decrease in COUP-TFII-expression in human coronary arterial ECs, indicating a possible mechanism through which VEGF-signaling might favor arterial endothelial differentiation. The exact pathway by which VEGF-signaling regulates COUP-TFII-expression remains to be elucidated.

The main isoform expressed during normal murine cardiac development is Vegf $164^{17}$. VEGF164 has a proximate bioactivity to its place of production due to high heparin-binding capacities $^{29}$ (Figure $1 Q$ and $5 A, C, E$ ). We show that cardiac Vegf-production in vivo overlaps in later stages ( $\geq E 16.5$ ) with the region where coronary arteries develop (i.e. deep in the myocardium of the ventricular free walls in the mouse). We consider that this spatial distribution will result in relatively high levels of VEGF and subsequent Notch-signaling in ECs localized in the region of the developing coronary arteries due to 1 ) high local levels of VEGF, combined with 2) amplification of signaling levels by artery-specific NP-1 ${ }^{28}$ (Figure $5 \mathrm{C}$ ). This is reflected in vivo by inhibition of COUP-TFII and induction of Dll4, cleaved Notch1 and ephrinB2 staining in coronary arterial ECs in late embryonic stages, suggesting increased Notch-signaling, and (potentially Notch-induced ${ }^{26}$ ) reduced VEGFR-2 expression. In contrast, veins develop subepicardially and therefore, venous ECs are mainly subjected to the less expressed and more diffuse VEGF120-isoform, with resulting low levels of Notch-signaling as indicated by low cleaved Notch1 and high COUP-TFII and EphB4-staining in wild type embryos. In combination with the fact that venous ECs do not express NP- $1^{28}$, this leads to the assumption that coronary venous ECs experience lower levels of VEGF and Notch-signaling (Figure 5E). This spatiotemporal determination of arterial and venous specification also corresponds with the situation in zebrafish. Here, VEGF-expression is induced in the somites next to the notochord, which is located directly caudal from the dorsal aorta. The 
posterial cardinal vein is positioned more ventrally, so further away from the place of VEGF-production ${ }^{27}$.

In Vegf120/120 mouse embryos, only the highly soluble non-heparin-binding VEGF120 isoform is produced ${ }^{29}$. This will affect the VEGF-gradient in the myocardium giving relative low levels of VEGF in the area of the coronary arteries and higher levels around the coronary veins (Figure 5B,D,F). Because of this, combined with the fact that VEGF120 cannot induce formation of the signaling potent NP-1/VEGFR-2 complexes ${ }^{30}$, lower VEGF and Notch-signaling levels are expected in the ECS in the area of the coronary arteries (Figure 5D), and higher levels in the ECs of the coronary veins (Figure 5F). This could lead to the alterations in EC-differentiation observed in these embryos. Besides altered differentiation, a severe decrease in coronary arterial size coincides with an increase in venous size. As VEGF is involved in endothelial proliferation and vascular fusion ${ }^{31}$, locally increased Vegf-expression in the venous region could lead to enlarged veins as observed in the mutant mouse embryos. Therefore, we speculate that the RT-qPCR data normalized for endothelial input on embryonic hearts indicate mainly venous expression. This underscores that VEGF-signaling in venous ECs might induce expression of arterial markers such as Notch1 and Notch4. Unfortunately, we could not confirm alterations in Notch4 protein-expression as the anti-Notch4-antibodies tested bind aspecific (Table S1).

Our in vitro data show that the expression of arterial markers in HCAECs varies for the VEGF-isoform applied, with VEGF165 having a more prominent effect than VEGF121. This probably relates to higher levels of VEGF-signaling through VEGFR-2 with VEGF165 due to NP-1-mediated amplification ${ }^{9,28,30}$. Unfortunately, we could not directly test the effect of VEGF-signaling on coronary venous ECs in vitro, as these cells cannot be obtained for culturing. However, earlier published data on HUVEC show that treatment with VEGF induces arterial phenotype of venous ECs ${ }^{11,}$ 32 as does ectopic VEGF-expression in zebrafish ${ }^{33}$.

Total heart volumes are unchanged in mutant embryonic hearts, while a decrease in myocardial/vascular volume-ratio is seen, together with abnormal (dilated) microvascular morphology. This pleads for an increase in vascular volume rather than a decrease in myocardial volume as the underlying cause for the altered myocardial/vascular volume-ratio. This is likely caused by a disturbed VEGFgradient, as it has been described that loss of a VEGF-gradient leads to alterations in microvascular branching morphogenesis ${ }^{34}$ and increased availability of VEGF induces hyperfusion of vessels ${ }^{31}$. Furthermore, VEGF is able to induce proliferation of lymphatic $\mathrm{ECs}^{35}$, located subepicardially in the heart. Another function of VEGF as Vascular Permeability Factor $^{36}$ might additionally lead to the appearance of the increased size of the cardiac lymphatic system in mutant embryos. 


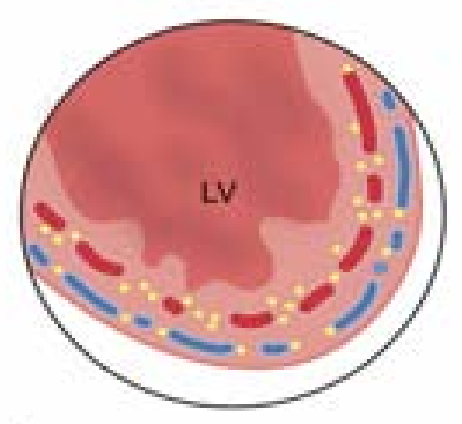

A.

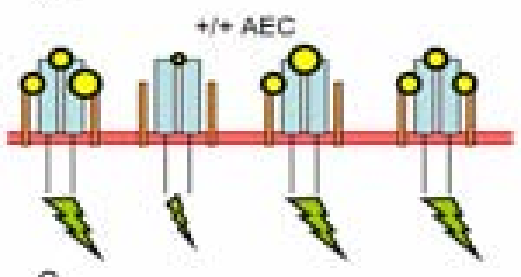

C

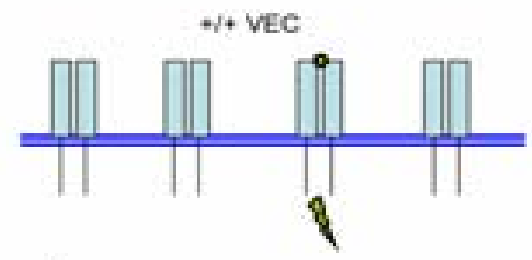

E

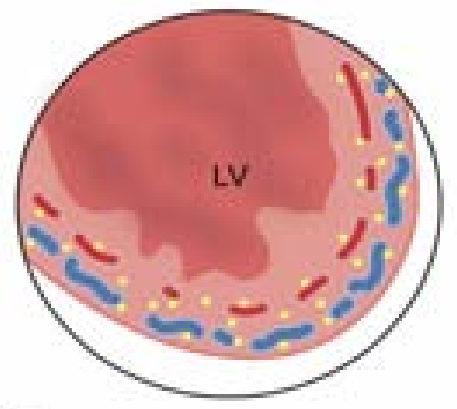

B

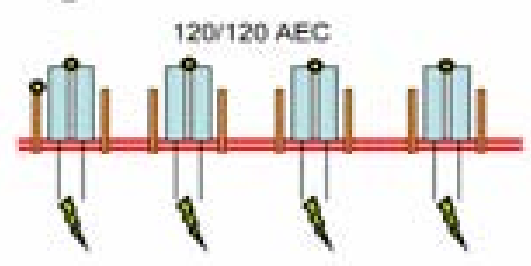

D

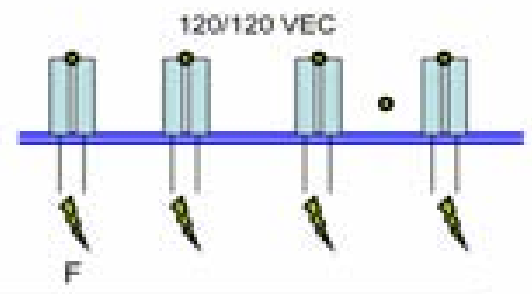

Figure 5. VEGF-expression and signaling during embryonic coronary development in normal and Vegf120/120 hearts. A-B: Schematic drawings of transverse sections of the left ventricle (LV) of wild type $(+/+)$ and mutant (120/120) embryos of E16.5 are shown. In +/+ embryos, Vegf-production mainly takes place in the borderzone between compact and trabecular myocardium (A; see also Figure $1 Q$ ), where coronary arteries (red) develop. As this is mainly Vegf164, protein distribution (yellow) is expected to give high protein levels around arteries and low around veins (blue). As mutants (B) only produce Vegf120, protein levels are expected to be more diffuse due to lack of heparin-binding domains. This will lead to relative low protein levels around arteries and high levels around veins. C-F: Schemes indicating the expected VEGF-signaling levels in $+/+$ and 120 coronary arterial endothelial cells (AECs) and venous endothelial cells (VECs) are shown. In a +/+ AEC high VEGF protein-levels combined with neuropilin-1 (NP-1)-mediated amplification leads to high levels of VEGF-signaling (C), while low VEGF-concentrations and lack of NP-1-expression in VECs leads to low levels of VEGFsignaling (E). In mutant embryos, relative low VEGF-levels around the AECs in combination with lack of NP-1-mediated amplification leads to relative low levels of VEGF-signaling in a 120/120 AEC (D), while relative high VEGF-levels around the VECs lead to high signaling levels (F). NOTE: Due to resolution, in A different VEGF-isoform are indicated with yellow dots of the same size. 


\section{Alterations in Vegf120/120 coronary arteriogenesis}

We show that incorrect coronary arterial EC-differentiation coincides with impaired development of the media, together with a gain of arterial phenotype in coronary venous ECs and an increased number of venous pericytes. The differentiational state of ECs influences recruitment or proliferation and maturation ${ }^{37,38}$, suggesting a role for VEGF-signaling on arteriogenesis via manipulation of endothelial performance. A direct role for VEGF-signaling in arteriogenesis is supported by the described instructive role for NP- $1^{39}$ and Notch-signaling ${ }^{38,40}$ in differentiation of vSMCs. Concomitantly, we show that the amount of cells expressing aSMA, NP-1, DIl1, Jagged2 and Notch3 in the coronary arterial media and of Notch3-positive microvascular pericytes was impaired in mutant embryos, while an increase in number of cells surrounding the coronary veins positive for these markers was seen. Besides a positive role for Notch-signaling in arteriogenesis, inhibiting roles have been described as well ${ }^{41}$. Our data plead for an instructive rather that an inhibiting role. We suggest that lack of larger VEGF-isoforms leads to a deficient development of the vSMC-layer of the coronary arteries. This can occur either indirectly via altered endothelial differentiation, or directly through a decrease in VEGF-related Notch-signaling in the mutant coronary vSMCs.

\section{Morphological abnormalities in the coronary system of mutant mouse embryos}

Congenital coronary anomalies are often correlated with primary cardiac defects. In all Vegf120/120 mutants with TOF $^{17}$ the IVS was supplied by an artery coming from the left sinus, in contrast to normal where the IVS is supplied by a branch of the right coronary artery ${ }^{24}$. The anomalies specific for TOF (overriding and/or dextroposition of the ascending aorta) might favor the coronary IVS-branch to connect to the left sinus ${ }^{42}$. As in humans with TOF, a single orifice was occasionally encountered in mutant mouse embryos. More often an increased number (3 or 4) of coronary orifices was found, which is regarded to be a rare variation in both humans and mice ${ }^{24,43}$. Nevertheless, we never saw more than 2 orifices in the 24 wild type embryos of E15.5 and older.

The presence of VCACs in cardiac anomalies related to pulmonary atresia are not uncommon, even before complete atresia has developed ${ }^{44}$. A substantial part of coronary vSMCs originates from the EPDC-population ${ }^{15}$ and EPDC-performance has been suggested to play part in the appearance of $\mathrm{VCACs}^{44}$. Abnormal development and/or differentiation of EPDCs due to altered VEGF and/or Notch-signaling might explain the occurrence of VCACs in Vegf120/120 embryos. Besides VCACs, coronary arterio-venous shunts were observed. Alterations in VEGF and Notch-signaling in Vegf120/120 embryos may cause arterio-venous shunts, as the presence of such shunts has been described in Notch-mutants ${ }^{45}$. Extracardiac arterio-venous shunts were observed in the aortic arches of perinatal Vegf120/120 mouse embryos, suggesting that alterations in Notch-signaling are not heart-restricted. 
We already observed at E11.5, both in wild type and mutants, two connections of the coronary venous system (in)directly to the right atrium. This is the first report on such early coronary venous drainage in mice. We describe that the connection of the coronary system to the right atrium arises before the coronary connection to the aorta. This is feasible as it enables circulation throughout the coronary system at the onset of systemic flow and pressure, which occurs several days later, without obstruction $^{14}$.

\section{Conclusions}

We show that loss of Vegf164 and Vegf188 in Vegf120/120 embryos leads to spatiotemporal alterations in VEGF and Notch-signaling in the heart reflected by altered expression of several markers involved in and regulated by these pathways. These alterations coincide with anomalous coronary EC-differentiation and ensuing arteriogenesis. The effect of different VEGF-isoforms on Notch-signaling and coronary EC-differentiation could be confirmed in vitro.

From our study it can be concluded that the spatiotemporal VEGF-distribution as well as specificity of the isoforms are important for proper coronary vascular development. These new insights on instructive roles of VEGF(-isoform) distribution on angiogenesis, endothelial functioning, and arteriogenesis can be of use to improve the efficacy of current therapies targeting VEGF-signaling ${ }^{8,46}$ in tissue revascularization.

\section{Acknowledgments}

The authors would like to thank Jan Lens for photographic assistance, Ron Slagter for drawings, Saskia Maas and Conny J. van Munsteren for technical assistance (all Department of Anatomy and Embryology, Leiden University Medical Center, Leiden, The Netherlands) and Sanne Verbruggen (Department of Vascular Physiology, Maastricht University, Maastricht, The Netherlands) for assistance with the in vitro experiments.

\section{Funding}

The Netherlands Heart Foundation (2001B057 to N.M.S.v.d.A.); The Marie Curie FP6 early stage researcher training (MEST-CT-20005-020706 to V.C.). 


\section{References}

1. Chi JT, Chang HY, Haraldsen G, Jahnsen FL, Troyanskaya OG, Chang DS, et al. Endothelial cell diversity revealed by global expression profiling. Proc Natl Acad Sci U S A 2003;100:10623-10628.

2. Kattan J, Dettman RW, Bristow J. Formation and remodeling of the coronary vascular bed in the embryonic avian heart. Dev Dyn 2004;230:34-43.

3. Casanello P, Escudero C, Sobrevia L. Equilibrative nucleoside (ENTs) and cationic amino acid (CATs) transporters: implications in foetal endothelial dysfunction in human pregnancy diseases. Curr Vasc Pharmacol 2007;5:69-84.

4. Gittenberger-De Groot AC, Van Den Akker NM, Bartelings MM, Webb S, Van Vugt JM, Haak MC. Abnormal lymphatic development in trisomy 16 mouse embryos precedes nuchal edema. Dev Dyn 2004;230:378-384.

5. Carson-Walter EB, Hampton J, Shue E, Geynisman DM, Pillai PK, Sathanoori R, et al. Plasmalemmal vesicle associated protein-1 is a novel marker implicated in brain tumor angiogenesis. Clin Cancer Res 2005;11:7643-7650.

6. Deng DX, Tsalenko A, Vailaya A, Ben-Dor A, Kundu R, Estay I, et al. Differences in vascular bed disease susceptibility reflect differences in gene expression response to atherogenic stimuli. Circ Res 2006;98:200-208.

7. Lauth M, Cattaruzza M, Hecker M. ACE inhibitor and AT1 antagonist blockade of deformationinduced gene expression in the rabbit jugular vein through B2 receptor activation. Arterioscler Thromb Vasc Biol 2001;21:61-66.

8. Yla-Herttuala S, Rissanen TT, Vajanto I, Hartikainen J. Vascular endothelial growth factors: biology and current status of clinical applications in cardiovascular medicine. J Am Coll Cardiol 2007;49:1015-1026.

9. Soker S, Takashima S, Miao HQ, Neufeld G, Klagsbrun M. Neuropilin-1 is expressed by endothelial and tumor cells as an isoform-specific receptor for vascular endothelial growth factor. Cell 1998;92:735-745.

10. Shawber CJ, Kitajewski J. Notch function in the vasculature: insights from zebrafish, mouse and man. Bioessays 2004;26:225-234.

11. Hainaud P, Contreres JO, Villemain A, Liu LX, Plouet J, Tobelem G, et al. The Role of the Vascular Endothelial Growth Factor-Delta-like 4 Ligand/Notch4-Ephrin B2 Cascade in Tumor Vessel Remodeling and Endothelial Cell Functions. Cancer Res 2006;66:8501-8510.

12. Villa N, Walker L, Lindsell CE, Gasson J, Iruela-Arispe ML, Weinmaster G. Vascular expression of Notch pathway receptors and ligands is restricted to arterial vessels. Mech Dev 2001;108:161-164.

13. Vrancken Peeters MP, Gittenberger-De Groot AC, Mentink MM, Hungerford JE, Little CD, Poelmann $\mathrm{RE}$. The development of the coronary vessels and their differentiation into arteries and veins in the embryonic quail heart. Dev Dyn 1997;208:338-348.

14. Vrancken Peeters MP, Gittenberger-De Groot AC, Mentink MM, Hungerford JE, Little CD, Poelmann RE. Differences in development of coronary arteries and veins. Cardiovasc Res 1997;36:101-110.

15. Vrancken Peeters MP, Gittenberger-De Groot AC, Mentink MM, Poelmann RE. Smooth muscle cells and fibroblasts of the coronary arteries derive from epithelial-mesenchymal transformation of the epicardium. Anat Embryol (Berl) 1999;199:367-378.

16. Stalmans I, Lambrechts D, De Smet F, Jansen S, Wang J, Maity S, et al. VEGF: a modifier of the del22q11 (DiGeorge) syndrome? Nat Med 2003;9:173-182.

17. Van Den Akker NM, Molin DG, Peters PP, Maas S, Wisse LJ, van BR, et al. Tetralogy of fallot and alterations in vascular endothelial growth factor-A signaling and notch signaling in mouse embryos solely expressing the VEGF120 isoform. Circ Res 2007;100:842-849.

18. Carmeliet P, Ng YS, Nuyens D, Theilmeier G, Brusselmans K, Cornelissen I, et al. Impaired myocardial angiogenesis and ischemic cardiomyopathy in mice lacking the vascular endothelial growth factor isoforms VEGF164 and VEGF188. Nat Med 1999;5:495-502.

19. Stalmans I, Ng YS, Rohan R, Fruttiger M, Bouche A, Yuce A, et al. Arteriolar and venular patterning in retinas of mice selectively expressing VEGF isoforms. J Clin Invest 2002;109:327-336. 
20. Gundersen HJ, Jensen EB. The efficiency of systematic sampling in stereology and its prediction. J Microsc 1987;147:229-263.

21. Hellemans J, Mortier G, De PA, Speleman F, Vandesompele J. qBase relative quantification framework and software for management and automated analysis of real-time quantitative PCR data. Genome Biol 2007;8:R19.

22. Uyttendaele H, Marazzi G, Wu G, Yan Q, Sassoon D, Kitajewski J. Notch4/int-3, a mammary protooncogene, is an endothelial cell-specific mammalian Notch gene. Development 1996;122:22512259.

23. Viragh S, Challice CE. Origin and differentiation of cardiac muscle cells in the mouse. J Ultrastructure Research 1973;42:1-24.

24. Kumar D, Hacker TA, Buck J, Whitesell LF, Kaji EH, Douglas PS, et al. Distinct mouse coronary anatomy and myocardial infarction consequent to ligation. Coron Artery Dis 2005;16:41-44.

25. Tomanek RJ, Ratajska A, Kitten GT, Yue X, Sandra A. Vascular endothelial growth factor expression coincides with coronary vasculogenesis and angiogenesis. Dev Dyn 1999;215:54-61.

26. Williams CK, Li JL, Murga M, Harris AL, Tosato G. Up-regulation of the Notch ligand Delta-like 4 inhibits VEGF-induced endothelial cell function. Blood 2006;107:931-939.

27. Lawson ND, Vogel AM, Weinstein BM. sonic hedgehog and vascular endothelial growth factor act upstream of the Notch pathway during arterial endothelial differentiation. Dev Cell 2002;3:127-136.

28. You LR, Lin FJ, Lee CT, Demayo FJ, Tsai MJ, Tsai SY. Suppression of Notch signalling by the COUP-TFII transcription factor regulates vein identity. Nature 2005;435:98-104.

29. Houck KA, Leung DW, Rowland AM, Winer J, Ferrara N. Dual regulation of vascular endothelial growth factor bioavailability by genetic and proteolytic mechanisms. J Biol Chem 1992;267:2603126037.

30. Pan Q, Chanthery Y, Wu Y, Rahtore N, Tong RK, Peale F, et al. Neuropilin-1 binds to VEGF121 and regulates endothelial cell migration and sprouting. J Biol Chem 2007;282:24049-24056.

31. Drake CJ, Little CD. VEGF and vascular fusion: implications for normal and pathological vessels. J Histochem Cytochem 1999;47:1351-1356.

32. Liu ZJ, Shirakawa T, Li Y, Soma A, Oka M, Dotto GP, et al. Regulation of Notch1 and DII4 by vascular endothelial growth factor in arterial endothelial cells: implications for modulating arteriogenesis and angiogenesis. Mol Cell Biol 2003;23:14-25.

33. Lawson ND, Scheer N, Pham VN, Kim CH, Chitnis AB, Campos-Ortega JA, et al. Notch signaling is required for arterial-venous differentiation during embryonic vascular development. Development 2001;128:3675-3683.

34. Ruhrberg C, Gerhardt H, Golding M, Watson R, loannidou S, Fujisawa H, et al. Spatially restricted patterning cues provided by heparin-binding VEGF-A control blood vessel branching morphogenesis. Genes Dev 2002;16:2684-2698.

35. Nagy JA, Vasile E, Feng D, Sundberg C, Brown LF, Detmar MJ, et al. Vascular permeability factor/vascular endothelial growth factor induces lymphangiogenesis as well as angiogenesis. J Exp Med 2002;196:1497-1506.

36. Brown LF, Detmar M, Claffey K, Nagy JA, Feng D, Dvorak AM, et al. Vascular permeability factor/vascular endothelial growth factor: a multifunctional angiogenic cytokine. EXS 1997;79:233269.

37. Carmeliet P. Mechanisms of angiogenesis and arteriogenesis. Nat Med 2000;6:389-395.

38. Limbourg A, Ploom M, Elligsen D, Sorensen I, Ziegelhoeffer T, Gossler A, et al. Notch ligand Delta-like 1 is essential for postnatal arteriogenesis. Circ Res 2007;100:363-371.

39. Liu W, Parikh AA, Stoeltzing O, Fan F, McCarty MF, Wey J, et al. Upregulation of neuropilin-1 by basic fibroblast growth factor enhances vascular smooth muscle cell migration in response to VEGF. Cytokine 2005;32:206-212.

40. High FA, Zhang M, Proweller A, Tu L, Parmacek MS, Pear WS, et al. An essential role for Notch in neural crest during cardiovascular development and smooth muscle differentiation. J Clin Invest 2007;117:353-363. 
41. Morrow D, Scheller A, Birney YA, Sweeney C, Guha S, Cummins PM, et al. Notch-mediated CBF1/RBP-J\{kappa\}-dependent regulation of human vascular smooth muscle cell phenotype in vitro. Am J Physiol Cell Physiol 2005;289:C1188-C1196.

42. Mawson JB. Congenital heart defects and coronary anatomy. Tex Heart Inst J 2002;29:279-289.

43. Angelini P. Coronary artery anomalies: an entity in search of an identity. Circulation 2007;115:12961305.

44. Gittenberger-De Groot AC, Eralp I, Lie-Venema H, Bartelings MM, Poelmann RE. Development of the coronary vasculature and its implications for coronary abnormalities in general and specifically in pulmonary atresia without ventricular septal defect. Acta Paediatr Supp/ 2004;93:13-19.

45. Krebs LT, Shutter JR, Tanigaki K, Honjo T, Stark KL, Gridley T. Haploinsufficient lethality and formation of arteriovenous malformations in Notch pathway mutants. Genes Dev 2004;18:2469-2473.

46. Gaffney MM, Hynes SO, Barry F, O'brien T. Cardiovascular gene therapy: current status and therapeutic potential. Br J Pharmacol 2007;152:175-188. 


\section{Supplement}

\section{Results}

Anomalous coronary morphology in Vegf120/120 mouse embryos

A remarkable observation was that half of the mutant embryos showed abnormalities in the anatomy of the main coronary branches. In all wild type embryos the main artery supplying the interventricular septum (IVS) originated from the first branch of the right coronary artery (RCA), with sometimes an additional smaller branch coming from the left coronary artery (LCA), which corresponds with earlier observations in normal mice ${ }^{1}$. In 8/28 mutant embryos the IVS-supplying branch had its own coronary orifice, which originated in 6 cases from the right and in 2 cases from the left facing sinus. In addition, in 3/28 cases the IVS was solely supplied by a branch that arose from the LCA. All embryos in which the IVS-branch originated (in)directly from the left facing sinus were diagnosed with TOF $(5 / 28)^{2}$. In contrast to mice, the IVS in humans is normally supplied by the left anterior descending artery and it has been described that patients with TOF often (up to $19 \%^{3}$ ) present with coronary abnormalities such as bilateral or rightoriginating anterior descending arteries ${ }^{3,4}$. The differences between mouse and man, both in normal and pathological coronary morphology might lie in the fact that the angle of the aorta relative to the IVS-axis differ between the two species ${ }^{5}$. Furthermore, in 2/28 Vegf120/120 cases no major coronary artery could be observed supplying the IVS and in $1 / 28$ cases both a branch splitting from the RCA and from the LCA with the left branch being most prominent was observed. 
Table S1. Characteristics of primary antibodies used for immunohistochemistry.

\begin{tabular}{|c|c|c|c|c|c|}
\hline Antigen & Cat. no. (company) & $\begin{array}{l}\text { Antigen } \\
\text { re- } \\
\text { trieval }\end{array}$ & $\begin{array}{l}\text { ABC- } \\
\text { incubation }\end{array}$ & $\begin{array}{l}\text { CSA-II kit } \\
\text { (DAKO) }\end{array}$ & Reference \\
\hline VEGFR-2 & $\begin{array}{l}\text { AF644 (R\&D } \\
\text { Systems, } \\
\text { Minneapolis, USA) }\end{array}$ & HR & Yes & No & $\begin{array}{l}\text { www.rndsystems.com } \\
\text { /ihc }\end{array}$ \\
\hline LYVE-1 & $\begin{array}{l}\text { 103-PABi50 } \\
\text { (ReliaTech, } \\
\text { Braunschweig, } \\
\text { Germany) }\end{array}$ & $P$ & Yes & No & Mäkinen et $a l^{6}$ \\
\hline $\begin{array}{l}\text { cleaved } \\
\text { Notch1 }\end{array}$ & $\begin{array}{l}2421 \text { (Cell Signaling, } \\
\text { Beverly, MA, USA) }\end{array}$ & HR & No & Yes & Tanaka et $a l^{7}$ \\
\hline Notch2 & $\begin{array}{l}\text { sc-5545 (Santa Cruz, } \\
\text { Santa Cruz, USA) }\end{array}$ & - & Yes & No & Okuyama et $a l^{8}$ \\
\hline Notch3 & $\begin{array}{l}\text { sc-7424 (Santa Cruz, } \\
\text { Santa Cruz, USA) }\end{array}$ & HR & Yes & No & Karlstöm et $a l^{9}$ \\
\hline Jagged1 & $\begin{array}{l}\text { sc-6011 (Santa Cruz, } \\
\text { Santa Cruz, USA) }\end{array}$ & HR & Yes & No & Sestan et $\left.a\right|^{10}$ \\
\hline Jagged2 & $\begin{array}{l}\text { sc-8158 (Santa Cruz, } \\
\text { Santa Cruz, USA) }\end{array}$ & - & Yes & No & Rivolta et $a l^{11}$ \\
\hline DII1 & $\begin{array}{l}\text { sc-9102 (Santa Cruz, } \\
\text { Santa Cruz, USA) }\end{array}$ & HR & Yes & No & Conboy and Rando ${ }^{12}$ \\
\hline DII4 & $\begin{array}{l}\text { AF1389 (R\&D } \\
\text { Systems, } \\
\text { Minneapolis, USA) }\end{array}$ & HR & Yes & No & www.rndsystems.com \\
\hline ephrinB2 & $\begin{array}{l}\text { AF496 (R\&D } \\
\text { Systems, } \\
\text { Minneapolis, USA) }\end{array}$ & HR & Yes & No & $\begin{array}{l}\text { www.rndsystems.com } \\
\text { /ihc }\end{array}$ \\
\hline EphB4 & $\begin{array}{l}\text { AF446 (R\&D } \\
\text { Systems, } \\
\text { Minneapolis, USA) }\end{array}$ & HR & Yes & No & www.rndsystems.com \\
\hline COUP-TFII & $\begin{array}{l}\text { PP-H7147- } \\
\text { 00,2ZH7147H } \\
\text { (PPMX, Tokyo, } \\
\text { Japan) }\end{array}$ & HR & Yes & No & You et $a l^{13}$ \\
\hline NP-1 & $\begin{array}{l}\text { sc-7239 (Santa Cruz, } \\
\text { Santa Cruz, USA) }\end{array}$ & HR & Yes & No & Serini et $a l^{14}$ \\
\hline $\begin{array}{l}\alpha-S M A \\
(1 \mathrm{~A} 4)\end{array}$ & $\begin{array}{l}\text { A2547 } \quad \text { (Sigma- } \\
\text { Adrich, Missouri, } \\
\text { USA) }\end{array}$ & HR & No & No & Skalli et $a 1^{15}$ \\
\hline Notch4 & $\begin{array}{l}\text { sc-8645 (Santa Cruz, } \\
\text { Santa Cruz, USA) }\end{array}$ & N.A. & N.A. & N.A. & N.A. \\
\hline Notch4 & $\begin{array}{l}\text { sc-5594 (Santa Cruz, } \\
\text { Santa Cruz, USA) }\end{array}$ & N.A. & N.A. & N.A. & N.A. \\
\hline
\end{tabular}

Abbreviations; $\mathrm{HR}=$ heat retrieval; N.A. = not applicable; $\mathrm{P}=$ pronase treatment 
Table S2. Primer sequences used for RT-qPCR.

\begin{tabular}{|c|c|c|c|}
\hline Species & Gene & Forward & Reverse \\
\hline \multirow[t]{9}{*}{$\begin{array}{l}\text { Homo } \\
\text { Sapiens }\end{array}$} & B-ACTIN (BC002409) & $\begin{array}{l}5^{\prime}- \\
\text { ATCCTCACCCTGAAGTACCC- } \\
3^{\prime}\end{array}$ & $\begin{array}{l}\text { 5'-GGACTGTGGTCATGAGTCCT- } \\
3^{\prime}\end{array}$ \\
\hline & GAPDH (NM_002046) & $\begin{array}{l}5^{\prime}- \\
\text { GCCTCAAGATCATCAGCAAT - } \\
3^{\prime}\end{array}$ & $\begin{array}{l}\text { 5'-GGACTGTGGTCATGAGTCCT- } \\
3^{\prime}\end{array}$ \\
\hline & NP-1 (NM_003873) & $\begin{array}{l}\text { 5'-TGAGCCCTGTGGTTTATTC } \\
-3^{\prime}\end{array}$ & $\begin{array}{l}\text { 5'-CGTACTCCTCTGGCTTCTGG- } \\
3^{\prime}\end{array}$ \\
\hline & NOTCH1 (NM_017617) & $\begin{array}{l}5^{\prime}- \\
\text { CTGGAGGACCTCATCAACTC - } \\
3^{\prime}\end{array}$ & $\begin{array}{l}5^{\prime}-\text { TTCTTCAGGAGCACAACTGC } \\
-3^{\prime}\end{array}$ \\
\hline & NOTCH4 (NM_004557) & $\begin{array}{l}\text { 5- } \\
\text { GTGGGTATCTCTGCCAGTGT- } \\
\text { 3' }\end{array}$ & $\begin{array}{l}\text { 5'-CTCAGGTTTGGGAGTACAG- } \\
3^{\prime}\end{array}$ \\
\hline & DLL4 (NM_019074) & $\begin{array}{l}5^{\prime}- \\
\text { ACAACTTGTCGGACTTCCAG- } \\
3^{\prime}\end{array}$ & $\begin{array}{l}\text { 5'-CAGCTCCTTCTTCTGGTTTG- } \\
3^{\prime}\end{array}$ \\
\hline & HES1 (NM_005524) & $\begin{array}{l}5^{\prime}- \\
\text { CCAAAGACAGCATCTGAGCA- } \\
3^{\prime}\end{array}$ & 5'-GCCGCGAGCTATCTTTCTT-3' \\
\hline & $\begin{array}{l}\text { EPHRINB2 } \\
\text { (NM_004093) }\end{array}$ & $\begin{array}{l}5^{\prime}- \\
\text { GTTCCGAAGTGGCCTTATTT- } \\
3^{\prime}\end{array}$ & $\begin{array}{l}\text { 5'-CTCCGGTACTTCAGCAAGAG- } \\
3^{\prime}\end{array}$ \\
\hline & COUP-TFII (BC014664) & $\begin{array}{l}5^{\prime}- \\
\text { AGCAAGTGGAGAAGCTCAAG } \\
-3^{\prime}\end{array}$ & $\begin{array}{l}5^{\prime}-\text { CACATGGGCTACATCAGAGA } \\
-3^{\prime}\end{array}$ \\
\hline \multirow{9}{*}{$\begin{array}{l}\text { Mus } \\
\text { Musculus }\end{array}$} & B-actin (NM_007393) & $5^{\prime}$ - actcctatgtgggtgacgag $-3^{\prime}$ & $5^{\prime}$ - ccagatcttctccatgtcgt $-3^{\prime}$ \\
\hline & gapdh (BC083149) & $5^{\prime}$ - aagtggagattgttgccatc $-3^{\prime}$ & $5^{\prime}$ - cgtgagtggagtcatactgg $-3^{\prime}$ \\
\hline & Tie2 (X71426) & $5^{\prime}$ - gccatcaagaggatgaaaga $-3^{\prime}$ & $5^{\prime}$ - catgctcccaagagattgat $-3^{\prime}$ \\
\hline & Notch1 (NM_008714) & $5^{\prime}$ - agcctctccaccaatacctt $-3^{\prime}$ & $5^{\prime}$ - ggctggagctgtaagttctg $-3^{\prime}$ \\
\hline & Notch4 (NM_010929) & $5^{\prime}$ - agaggacgggactacacctt $-3^{\prime}$ & $5^{\prime}$ - ccttttatccctggctccta- $3^{\prime}$ \\
\hline & Hes1 (NM_008235) & $5^{\prime}$ - cctctgagcacagaaagtca $-3^{\prime}$ & $5^{\prime}$ - gccgggagctatctttctta $-3^{\prime}$ \\
\hline & Ephb4 (NM_010144) & $5^{\prime}$ - gccatcaagatgggaagata $-3^{\prime}$ & $5^{\prime}$ - cacactggccaagattttct $-3^{\prime}$ \\
\hline & DII4 (NM_019454) & $5^{\prime}$ - acttcgtctgcaactgtcct $-3^{\prime}$ & $5^{\prime}$ - cagcaccagcagtaccacta $-3^{\prime}$ \\
\hline & Hey1 (AF232241) & $5^{\prime}$ - gtacccagtgcctttgagaa $-3^{\prime}$ & $5^{\prime}$ - tttcaggtgatccacagtca $-3^{\prime}$ \\
\hline
\end{tabular}


Table S3. Overview of alterations in coronary EC-expression patterns in Vegf120/120 mouse embryos.

\begin{tabular}{lll}
\hline Marker & Venous ECs & Arterial ECs \\
\hline VEGFR-2 & $\uparrow$ & $\uparrow$ \\
cleaved Notch1 & $\uparrow$ & $\downarrow$ \\
Notch2 & $\uparrow$ & $=^{*}$ \\
Dll4 & $\uparrow$ & $\downarrow$ \\
Jagged1 & $=*$ & $\downarrow$ \\
ephrinB2 & $\uparrow$ & $\downarrow$ \\
EphB4 & $\downarrow$ & $\uparrow$ \\
COUP-TFII & $\downarrow$ & $\uparrow$ \\
\hline
\end{tabular}

The protein expression patterns as determined using immunohistochemistry of ECs in Vegf120/120 mouse embryos are indicated, when compared to wild type littermates, as shown in Figure 1, 2 and data not shown $\left({ }^{*}\right)$. To indicate a difference, this difference was seen in at least 3 mutant vs 3 wild type embryos per age-group (E15.5-E19.5). 



\section{Chapter 5}

\section{Soluble JAGGED-1 Inhibits Neointima}

Formation by Attenuating NOTCH-HERP2 Signaling

Vincenza Caolo ${ }^{1^{*}}$, Henny M. Schulten ${ }^{1^{*}}$, Zhen W. Zhuang ${ }^{2}$, Masahiro Murakami ${ }^{2}$, Allard Wagenaar ${ }^{1}$, Sanne Verbruggen ${ }^{1}$, Daniel G. M. Molin ${ }^{1}$, Mark J. Post ${ }^{1}$

${ }^{1}$ Department of Physiology, Cardiovascular Research Institute Maastricht (CARIM), Maastricht University, Universiteitssingel 50, 6229 ER Maastricht, The Netherlands;

${ }^{2}$ Section of Cardiovascular Medicine, Yale School of Medicine, New Haven, CT, USA Yale.

* Contributed equally to this manuscript

Arterioscler. Thromb. Vasc. Biol. 2011, In press. 


\begin{abstract}
Notch has been implicated in neointima formation as reflected by increased Notch/Jagged expression upon vascular injury and the pro-migratory effect of Notch signaling on smooth muscle cells (SMC). Soluble Jagged-1 (sJag1) has been shown to inhibit Notch-signaling in vitro, however its capacity to suppress neointima formation remains unknown. Balloon injury of rat carotid arteries induced Notch1, Notch 3 and Jagged-1 expression at day 3 and 14 post-injury. Notch signaling was activated as shown by increased expression of the Notch target gene Herp2. Adenoviral-sJag1 (Ad-sJag1) transfection reduced neointima formation in carotid artery and enhanced re-endothelialization, whereas adenoviral-full length Jagged-1 (Ad-Fl-Jag1) or -LacZ had no effect. Injury induced Herp2 expression was absent in vessels treated with Ad-sJag1. Consistently, Herp2 expression was reduced in AdsJag1 infected or recombinant sJag1 treated Coronary Artery Smooth Muscle Cells (CASMCs). Ad-sJag1 had no effect on Human Umbilical Endothelial Cells (HUVECs) behavior, but significantly reduced proliferation and migration of CASMCs. Overexpression of Herp2 in sJag1 treated CASMCs rescued the migratory and proliferative capacity in vitro. Our results demonstrate that sJag1 can inhibit neointima formation after balloon injury by decreasing SMC proliferation and migration through interference with Notch-Herp2 signaling.
\end{abstract}




\section{Introduction}

Occlusive arteriopathies, such as coronary artery and peripheral artery disease are a consequence of atherosclerosis related neointima formation involving maladapted migration and proliferation of smooth muscle cells (SMCs). As vascular SMCs are not terminally differentiated, they exhibit substantial plasticity in phenotypic modulation that can be activated upon a vascular insult ${ }^{1,2}$. Members of the Notch family can regulate SMC plasticity and have been associated with neointima formation $^{3-7}$.

The Notch family encompasses an evolutional highly conserved intercellular signaling pathway known to regulate cell fate decision (i.e. apoptosis, proliferation, differentiation and migration $)^{8}$ in the cardiovascular system ${ }^{9,10}$. This family consists of four Notch receptors (Notch1-4) and two ligand groups, Jagged (Jagged1, -2) and Delta Like (DII1, -3, -4). Both receptors and ligands are transmembrane proteins containing an extracellular domain with which they facilitate communication between cells. After binding of the ligand to the receptor -present on adjacent cellsa proteolytic cascade is activated involving the $\gamma$-secretase/ADAM (A Disintegrin And Metalloprotease) complex. Cleavage of the intracellular part of the receptor results in the release of the Notch intracellular domain (NICD) that translocates to the nucleus and together with its co-transcription factor CSL (CBF1/RBPJk, Su(H), Lag1) induces the transcription of the basis helix-loop-helix transcription factors HES (Hairy/Enhancer of Split) and HERP (Hairy Enhancer of Split-related Repressor Protein $)^{11-13}$. Also the ligand is cleaved upon receptor interaction by the same proteases, giving rise to a soluble extracellular ligand domain that can influence NOTCH signaling as well ${ }^{14}$. For example, soluble Jagged1 (sJag1) inhibits migration and proliferation of the fibroblast cell line $\mathrm{NIH} 3 \mathrm{~T}^{15,16}$.

Notch1, -3 and Jagged1 along with the downstream HES/HERP-proteins are expressed in adult SMCs ${ }^{17}$. Jagged1 and Notch1 play important non-redundant roles during vascular development as their respective knockouts die early in utero due to vascular remodeling defects of the yolk $\operatorname{sac}^{18,19}$. Notch 3 mutants are viable and fertile but present with structural vascular defects showing reduced arterial size and deficient SMC differentiation post natally (i.e. low SM22 $\alpha$ and smoothelin expression $)^{20,21}$, suggesting a function of Notch3 in vascular maturation. A role for Notch3 in arterial maturation is also supported by the phenotype of NOTCH3 mutations in men (i.e. CADASIL, Cerebral Autosomal Dominant Arteriopathy with Subcortical Infarcts and Leukoencephalopathy) ${ }^{22}$. In vitro experiments by Sweeney and colleagues underline this notion further, showing that constitutive expression of Notch1/3ICD (IntraCellular Domain) in SMCs increased proliferation and inhibited apoptosis ${ }^{6}$. Consistently, Jagged1 increased the expression of smooth musclemyosin heavy chain $(\mathrm{SM}-\mathrm{MHC})^{23}$, while the Notch down-stream transcription factors 
HERP1 and HERP2 were able to stimulate SMC migration, proliferation ${ }^{9}$ and differentiation ${ }^{24-26}$.

As migration of SMCs is a prerequisite for neointima formation after arterial injury, we hypothesized that blockage of Notch signaling with sJag1 could reduce neointima formation. Here, we show that neointima formation in a balloon injury carotid rat artery model was inhibited by sJag1. Accordingly, sJag1 was able to reduce the SMC proliferation and migration capacity, by interfering with Notch signaling involving depression of Herp2 expression.

\section{Methods}

A detailed description of methods can be found in the supplemental materials.

\section{Statistical analysis}

For inter-group comparison, one-way ANOVA with Tukey posthoc test using SPSS software was applied (version 12.0). Values are expressed as mean \pm SEM. Probability values of $<0.05$ were considered significant.

\section{Results}

\section{Notch1, Notch3, Jagged1 and Herp2 were Highly Expressed in Injured Arteries}

The expression of Notch1, Notch3, Jagged1 and Herp2 in intact and injured carotid arteries was determined at day 3 (early stage injury response) and day 14 (late stage injury response) post injury by immunohistochemistry and was compared to shams (Figure 1). Upon injury neointima formation progressed rapidly over time, beginning at day 3 and showing a profound and cell rich neointima at day 14 post injury (intima to media ratio $>1.7$ ). This injury response was associated with clear spatialtemporal changes in the expression of Notch members.

In non-injured carotid arteries, the Notch receptors (Figure $1 \mathrm{~A}$ ) were differentially expressed with Notch1 being present throughout the vessel wall, but more pronounced in the endothelium. Notch3 was expressed at a much lower level with moderate presence in the media and relative high signal in adventitial myofibroblasts. Jagged-1 was heterogeneously expressed with relative low levels in the intima and with individual cells containing a higher protein content in the media and adventitia. For Herp2 a relative low and scattered expression was apparent for the vessel wall with a higher level for the adventitia.

Three days after injury (Figure 1B), Notch1 was reduced throughout the wall with exception of the peri-adventitial layer of SMCs. In contrast, Notch3 was increased in the injured media as well as the adventitia with a typical high expression in the periendothelial SMC layer. Jagged-1 and Herp2 expression was also increased with a 


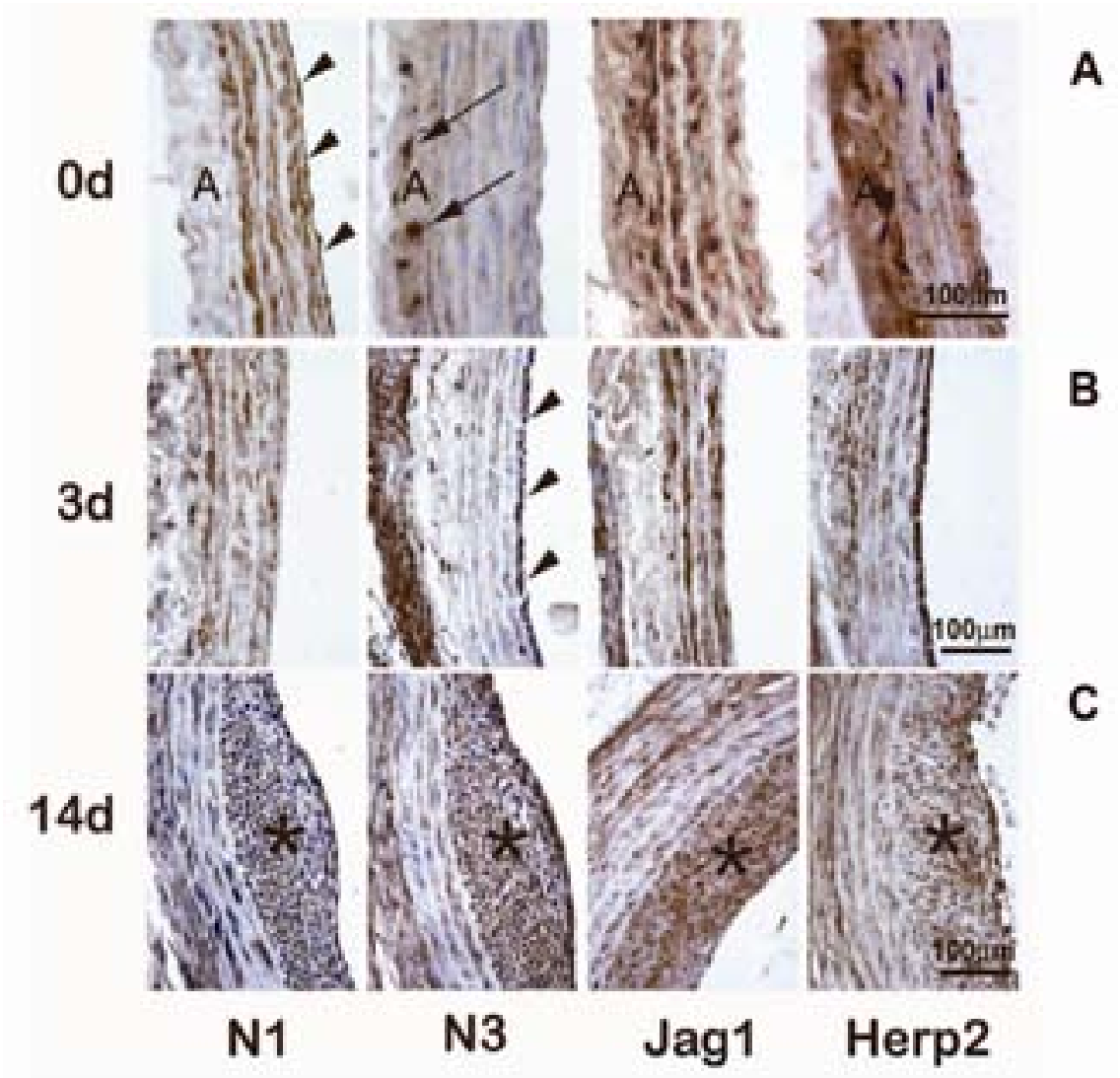

Figure 1. Notch1, Notch3, Jagged1 and Herp2 expression in non-injured (0d, A) and injured carotid arteries at three (3d, B) and fourteen days (14d, C) following injury. High magnification images of cross sections with similar orientation, i.e.lumen to the right and adventitia (A) to the left. A, At Od, Notch1 (N1) is expressed throughout the vessel wall, but especially in the endothelium (arrowheads). Notch3 (N3) is barely detectable in the media. Only adventitial myofibroblasts expressed N3 at high level (arrows). Jagged-1 (Jag1) and Herp2-expression was found in the vessel wall. B, At 3d, N1 expression was reduced and N3 was increased, showing a high content in adventitia and the more luminal SMC layers (arrowheads). Also Jag-1 and Herp2 were present in the wall, Jag-1 was expressed by all SMCs and the Herp2-pattern highly resembled N3. C, 14d after injury the neointima layer (asterisk) abundantly expressed all Notch members.

prominent signal in the peri-endothelial SMCs and adventitia, overlapping the Notch3 expression pattern. At day 14, the cell rich neointima expressed all Notch members stronger than the media and adventitia (Figure 1C). At this stage the expression of Herp2 remained high, reflecting a considerable degree of Notch signaling in the injured vessel wall. Interestingly, the vasa vasorum surrounding the vessel were highly Notch positive (not shown). 


\section{SJag1 negatively affected the Proliferation and Migration of SMCS}

To define the potential effects of sJag1 on EC and SMC behavior, both primary cell types were transduced with adenoviral vectors for either sJag1 (Ad-sJag1), Fulllength Jag1 (Ad-Fl-Jag1) or LacZ (Ad-LacZ, control), cultured and subsequently analyzed for their migratory and proliferative capacity (Figure 2). Standard microscopy on cell morphology and viability revealed no confounding cytotoxicity. Transduction efficiency was established by Western Blotting for c-myc and Jagged-1 on protein extracts from CASMCs and HUVECs that were infected with Ad-sJag1 and Ad-FIJag1, respectively or Ad-LacZ as control. All infected cells revealed clear increased expression of the protein of interest as compared to the LacZ control (Figure 2E and 2F and Suppl. Figure IIB).
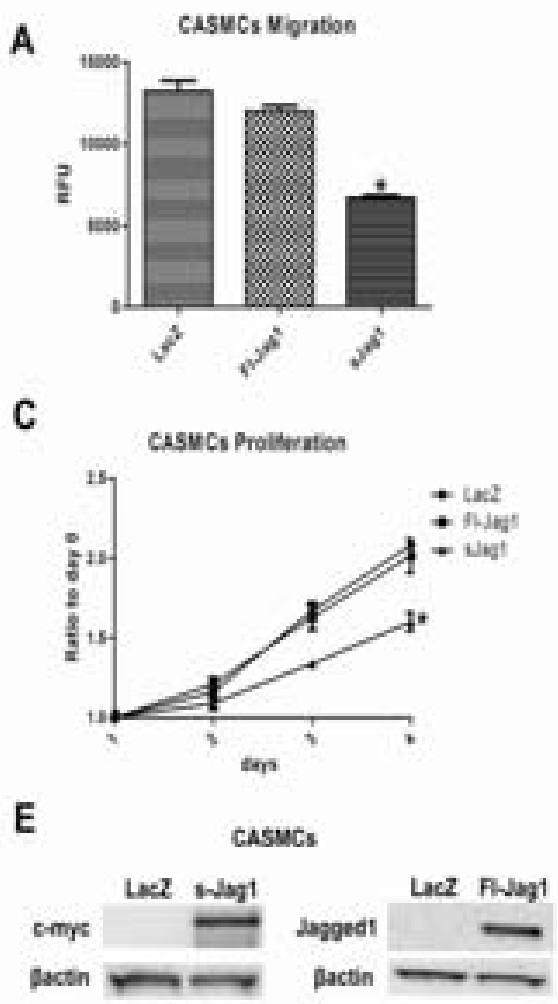

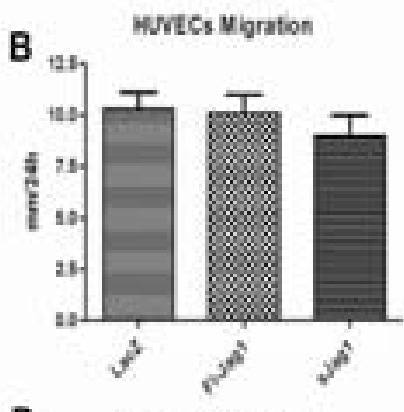

D

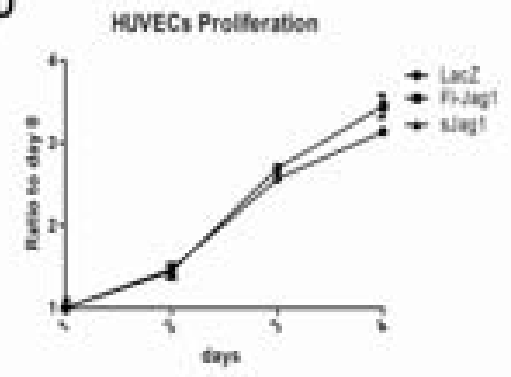

$\mathbf{F}$

HUVECS

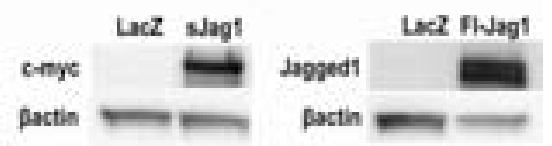

Figure 2. Effect of Jagged-1 on CASMCs and HUVECs function. A, Ad-sJag transduced CASMCs migrated at significant lower rate (asterisk, $\mathrm{p}<0.01$ ) compared with Ad-LacZ or Ad-Fl-Jag1 transduced CASMCS. B, HUVECS migration was unaffected by the treatments. C, CASMCs proliferation was significantly reduced by Ad-sJag1 (asterisk, $p<0.01$ ). D, Proliferation rate of HUVECs between treatments was comparable. E and F, Western blots of Ad-sJag1 and Ad-Fl-Jag1 transduced CASMCs and HUVECs showing clear protein over-expression. RFU (Relative Fluorescence Units). Data presented are mean values \pm SD of three independent experiments. 
Ad-sJag1, but not Ad-Fl-Jag, considerably reduced migration of CASMCs (Figure 2A

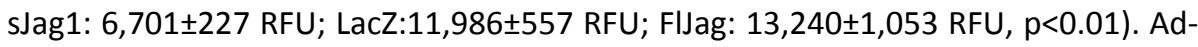
sJag1 also significantly depressed the proliferation of CASMCs as compared with AdLacZ and Ad-Fl-Jag1 infected cultures at both 48 (Figure $2 \mathrm{C}$ ratio to 24 hours: $1.34 \pm 0.024$ versus $1.67 \pm 0.051$ and $1.63 \pm 0.077$, respectively, $p<0.01$ ) and 72 hours post transduction $(1.60 \pm 0.059$ versus $2.08 \pm 0.024$ and $2.02 \pm 0.10$, respectively, $\mathrm{p}<0.01$ ). In contrast, migration and proliferation of endothelial cells (HUVECs) were unaffected by Ad-sJag1 or Ad-Fl-Jag (Figure 2B and 2D). A comparable lack of effect was also obtained with human coronary artery endothelial cells (data not shown). Interestingly, Lindner et al $^{16}$ reported that NIH3T3 cells stably transfected with soluble Jag1 migrated at a slower rate than cells transfected with a control vector. However, when cells were cultured on a fibronectin substrate this phenotype was partially rescued. To rule out matrix dependency of sJag induced effects we therefore tested the migration capacity of Ad-LacZ and Ad-sJag1 infected HUVECs that were cultured on fibronectin or plastic, but we were unable to detect a difference (Suppl. Figure IA and IB). Likewise, no difference in proliferation rate between sJag1 and LacZ infected HUVECs cultured on fibronectin was apparent (Suppl. Figure IC).

\section{sJag1 Reduced Neointima Formation}

To determine the effect of sJag1 on the development of neointima formation, injured carotid arteries were instilled with Ad-sJag1, Ad-Fl-Jag1 or Ad-LacZ control. In vivo efficiency defined by X-Gal staining of Ad-LacZ infected day 3 post injury rat carotid arteries was sufficient (Suppl. Figure IIA). The efficiency of infection was confirmed in cultured CASMCs infected with Ad-FI-Jag1 by immunofluorescent staining performed against the extracellular domain of Jagged1 protein (Suppl. Figure IIB).

Morphometrical analysis was performed on carotid artery cross sections 14 days after injury that were infected with Ad-sJag1, Ad-Fl-Jag1 or Ad-LacZ. Neointima formation in control Ad-LacZ treated carotid arteries was comparable to injured non-transduced carotid arteries, excluding cytotoxic effects of the viral intervention in vivo (data not shown). In accordance with the observed in vitro reduction in SMC proliferation and migration, Ad-sJag treatment resulted in more than $40 \%$ $(1.02 \pm 0.19)$ reduction in intima/media ratio compared with the control and Ad-FIJag1 $(1.74 \pm 0.18$ and $1.73 \pm 0.14$, respectively; $p<0.01)$ (Figure $3 A$ and $B$ ). Immunohistochemical staining against proliferating cell nuclear antigen (PCNA) was performed to examine the effect of sJag1 on SMC proliferation in vivo. Smooth muscle cells proliferative index at 3 days (calculated as the percentage of positive cells among total nucleated cells) was significantly lower in the media of injured carotid arteries from Ad-sJag1 group as compared with Ad-LacZ group ( $40 \pm 8 \%$ 
A

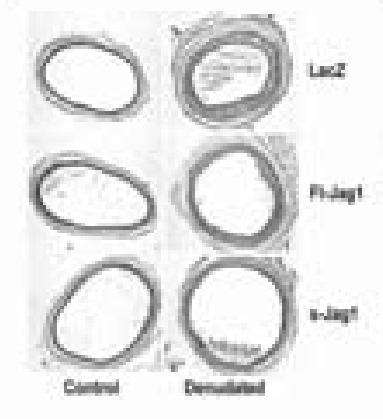

D

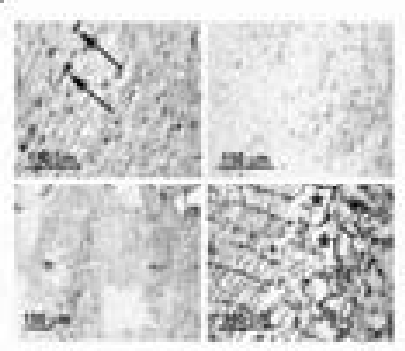

B

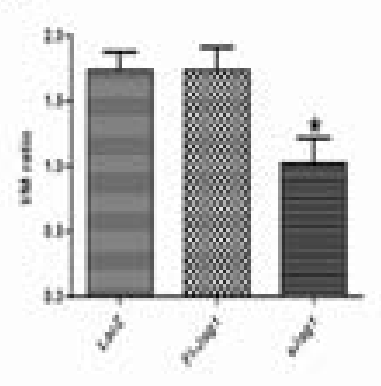

C

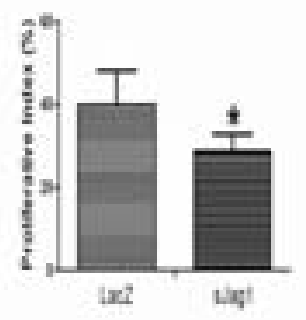

E

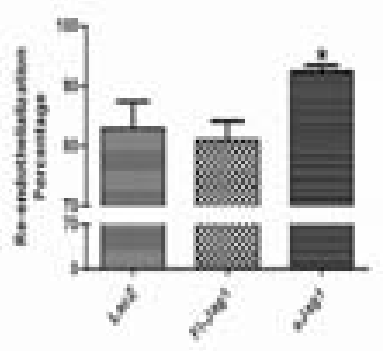

Figure 3. Effect of sJag1 and Fl-Jag on neointima formation. A, Eosin stained cross sections of noninjured (native; control) and denudated carotid arteries at 14 days post-injury. Smaller neointima was apparent for the Ad-sJag1 (lowest-panel) as compared with control (Ad-LacZ; upper-panel) and Ad-FlJag1 (middle-panel) B, Morphometric analysis showed significant lower average intima/media ratios (asterisk, $\mathrm{p}<0.05$ ) for the Ad-sJag1 as compared with both Ad-LacZ and Ad-Fl-Jag1 transduced arteries. C. Effect of in vivo sJag1 on proliferation as assessed by proliferating cell nuclear antigen staining (PCNA) staining. Proliferative index at 3 days after balloon injury and gene delivery was significantly lower for carotid artery section of Ad-sJag1 as compared with Ad-LacZ group. D, Representative en face $\mathrm{AgNO} 3$ stained carotid arteries showing the EC-borders in untreated control (upper left panel, black arrow points to EC mitosis); control immediately after balloon injury presenting complete removal of the endothelial layer (upper-right panel); 2 weeks post arterial injury with Ad-LacZ (lowerleft panel) revealing disorganized and incomplete endothelial coverage (asterisks) and Ad-sJag1 treatment with good endothelial recovery (lower right panel, asterisks indicating areas lacking endothelial recovery). E, Small though significant (asterisks, $p<0.05$ ) higher degree of reendothelialization was apparent for the Ad-sJag1 treated group at 14 days post injury.

versus $28.7 \pm 4.2 \%, p<0.05$; Figure $3 C$ ). No difference in SMC proliferation rate between treatments was observed at 14 days after injury. A significantly higher degree of reendothelialization was apparent for the Ad-sJag1 treated group after 14 days, whereas the Ad-Fl-Jag1 treated group did not differ from the control $(92.4 \% \pm 1.0 \%$ versus $81 \% \pm 3.3 \%$ and $83 \% \pm 4.4 \%$; Figure $3 \mathrm{E}$ and en face staining Figure 3D). Expression analysis of Notch members in the adenoviral treated groups revealed no clear differences in expression of Notch1 at day 3 and 14 day post- 
injury. As expected, Jag1 was higher expressed in the Ad-FlJag1 treated group at day 3 (Suppl. Figure III). For all other conditions and time points no differences in Jag1 expression were apparent. The majority of the 3 day Ad-sJag1 treated group (3 out of 4) revealed a lower Notch3 expression for the media (Suppl. Figure IV, B). This difference in expression was not found at day 14 post-injury. The degree of SMC differentiation of the injured carotid arteries was analyzed by examining the $\alpha$ smooth muscle actin ( $\alpha \mathrm{SMA}$ ) expression in the vessel wall. Three days after injury $\alpha$ SMA was observed in all SMC layers of the vessel wall. In contrast, the 3 day AdsJag1 treated vessels revealed a reduction in $\alpha \mathrm{SMA}$ expression as reflected by patches of low actin signal in the vessel wall (Suppl Fig V, C and D). At day 14 postinjury, strong aSMA expression was observed in the media and intima of injured vessels, with less clear and inconsistent differences between Ad-sJag1 and Ad-LacZ groups (Suppl. Figure V, E and F).

\section{Notch Related Herp2 Signaling in Neointima Formation and SMC function}

Treatment of injured carotid arteries with Ad-sJag1 resulted in a sharp decrease in medial expression of the Notch target gene Herp2 three days after injury (Figure 4A, right panel) compared to Ad-LacZ group (Fig.4A, left panel). In contrast, Ad-FI-Jag1 was unable to prevent the up-regulation of Herp2 expression induced by injury (Suppl. Figure VI A).

The inhibitory effect of sJag1 on Herp2 expression was further assessed in vitro. CASMCs treated with $5 \mu \mathrm{g} / \mathrm{mL}$ of sJag1 recombinant protein or infected with AdsJag1 decreased Herp2 expression (right and left panels, Figure 4B). Conversely, CASMCs infected with Ad-Fl-Jag1 showed increased Herp2 expression (Suppl. Figure VI B).

The functional relevance of sJag1 inhibited Herp2 for the migration and proliferation of SMC was studied by a rescue experiment in which Herp2 was over expressed. As expected, sJag1 gave a strong reduction in SMC migration in comparison with mock treated control for all time points analyzed (Figure 4C). At 24 hours a more than $10-$ fold difference between control and sJag1 treated control was apparent $(8.8 \%$ versus $100 \%$ ). This depressed SMC migratory capacity could partially be rescued by inducing Herp2 expression (52.5\% after $24 \mathrm{hrs}$ ), indicating that Notch-Herp2 signaling is involved in SMC migration. Similarly, the negative effect of sJag1 on CASMC proliferation was abrogated by Herp2 overexpression (Figure 4D). Soluble Jag1 treatment, at 72 hours post transfection, resulted in a significant reduction of SMC proliferation as compared to non treated mock condition (Ctrl) (Figure 4D ratio to 24 hours sJag1, $1.78 \pm 0.031$ versus Ctrl, $2.37 \pm 0.002 ; p<0.001)$. Herp2 overexpression was able to rescue SMC proliferation of the sJag1 treated samples (Herp2+sJag1, 2.65 \pm 0.018 versus sJag1, 1.78 $\pm 0.031 ; \mathrm{p}<0.001$ ), and increased the proliferation in untreated cells (Herp2, 3.22 \pm 0.005 versus Ctrl, 2.37 \pm 0.002 ; $\mathrm{p}<0.001)$. 


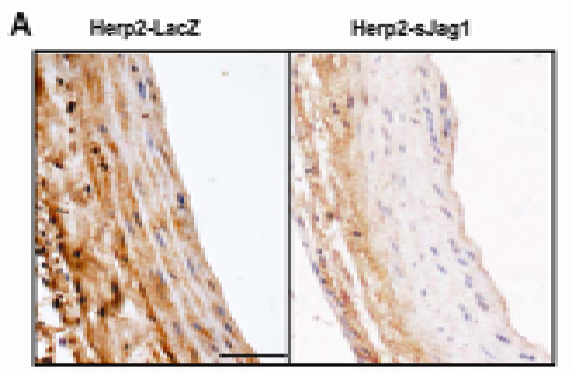

C

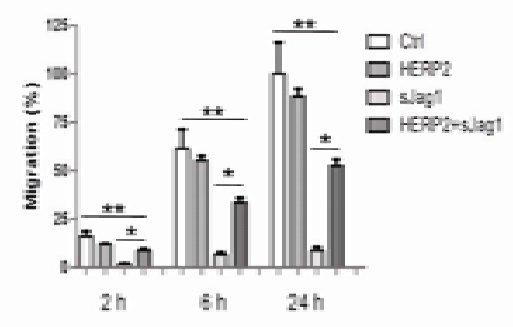

B

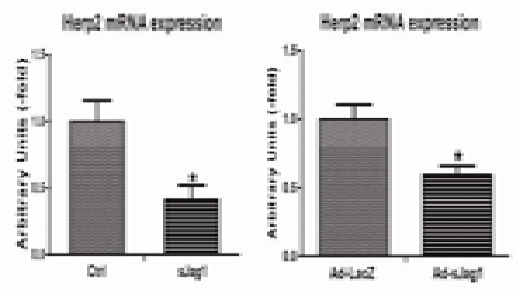

D

SuCs Prolferation

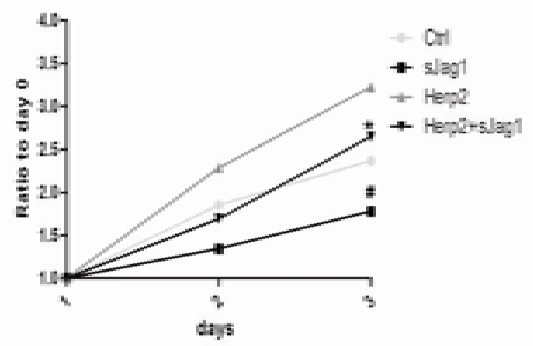

Figure 4. Cross section balloon injured and Ad-LacZ ( $A$, left panel) and Ad-sJag1 ( $A$, right panel) transduced carotid arteries showing Herp2 expression at 3 days post-injury. Ad-sJag treated arteries ( $A$, right panel) showed a decreased medial Herp2 expression as compared with Ad-LacZ treated arteries ( $A$, left panel). Scale bar: $200 \mu \mathrm{m}$. B. Herp2 mRNA expression in CASMCs receiving mock or recombinant sJag1 and CASMCs infected with Ad-sJag1 or Ad-LacZ control. Herp2 mRNA expression was significantly reduced by recombinant sJag1 (left panel). Similarly, Ad-sJag1 infected cells showed a significant decrease of Herp2 mRNA expression (right panel). Data presented as mean \pm SD of three independent experiments. C, Migration of Herp2- or Mock-transduced (control, ctrl) CASMCs treated with or without recombinant sJag1. Migration was depressed in CASMCs treated with sJag1 and Herp2 over-expression partly rescued this reduced migration capacity. Data presented as mean \pm SD of three independent experiment, values are normalized for mock group at 24 hours of migration and was set at $100 \%$. D. Proliferation of Herp2- or Mock-transduced (control, ctrl) CASMCs treated with or without recombinant sJag1. Proliferation was decreased (\#) in CASMCs the sJag1 group, whereas Herp2 overexpression rescued $(*)$ this effect. Data presented as mean \pm SD of three independent experiments. 


\section{Discussion}

In this study we present evidence that soluble Jag1 effectively inhibits neointima formation upon vascular injury through attenuation of Notch signaling and subsequent reduction of SMC proliferation and migration.

The development of intimal hyperplasia is associated with a temporal spatial change in Notch1, Notch3, Jagged1 and Herp2. Already three days post-injury a clear upregulation of Notch3, Jagged1 and Herp2 was obvious for the vessel wall, indicating a quick induction of Notch expression and activation upon insult at the locus of neointima formation. The up-regulation of Notch members is in agreement with previously published data ${ }^{4,16}$. However, two studies ${ }^{27,25}$ have reported a down regulation of Notch members at mRNA and protein level ${ }^{27}$ or low levels of Herp2 expression ${ }^{25}$ during the early phases of vessel remodeling. This discrepancy most likely relates to the region of the injured vessel examined as we focused on the border zone of the regenerating endothelium and mid-sections, whereas others analyzed the mRNA and protein content of the entire vesse ${ }^{27}$ or studied the aorta ${ }^{25}$. Neointima formation is considered the result of excessive SMC proliferation and migration that can be regulated and contained by regenerating endothelium ${ }^{28,29}$. The marked up-regulation of Jagged and Notch expression in the regenerating endothelium and underlying media of balloon injured rat carotid arteries point towards a localized Notch signaling circuitry that is activated upon vascular injury. The positive role of Notch signaling in SMCs proliferation and migration has been shown in models of constitutive overexpression of intracellular Notch1 and Notch $3^{6}$. Consistently, abrogated Notch signaling in hemizygous Notch1 and Herp1 knockouts reduced SMC proliferation and migration as well as neointima formation ${ }^{4}, 30$. Moreover, sJag1 is known to interfere with Notch signaling ${ }^{31,14}$ and to reduce cell migration and proliferation of NIH3T3 fibroblastic cells ${ }^{16}$. We showed that sJag1 is able to diminish SMC migration and proliferation in vitro. Accordingly proliferation of SMCs was reduced in the media of carotid artery from Ad-sJag1 group at day 3 as compared to Ad-LacZ group.

In vivo re-endothelialization was slightly increased at 14 days after vascular injury, whereas sJag1 treated ECs in vitro revealed no obvious changes in proliferation or migration. In view of the small in vivo difference found and the lack of a clear effect in vitro, it is unlikely that sJag1 plays a major role in endothelial regeneration. It is worth to note that Jag1 appears to be the main ligand for vascular SMCs related Notch signaling ${ }^{23}$, whereas DLL4 has been reported the major inducer of NOTCH activation in $\mathrm{EC}^{32,33}$. Accordingly, we show that stimulation of ECs with immobilized recombinant DLL4 increased the expression of the Notch target genes HES1, HERP1, -2 and -3 (Suppl. Figure VII and ${ }^{34}$ ). Conversely, stimulation with immobilized recombinant Jag1 had no effect on these Notch target genes in ECs except for HERP2. Nonetheless, this increase in HERP2 expression following recombinant Jag1 stimulation was far less profound than after recombinant DLL4 stimulation (Suppl. 
Figure VII). Thus, other Notch ligands, beyond Jagged1, are likely involved in regulating endothelial regeneration following vascular injury, with DLL4 being a possible candidate. Lindner et al $^{16}$ showed matrix protein dependency of sJag1 effects on NIH3T3. Specifically, NIH3T3 migrated at much slower rate than cells transfected with vector control when seeded on plastic, but on fibronectin this effect was much reduced. Therefore, we tested the effect of fibronectin coating on the migration and proliferation of EC in presence of sJag1. We found that HUVECS cultured on fibronectin failed to respond to sJag1 as HUVECs cultured on plastic, indicating that the lack of effect of sJag1 on ECs function is not related to the coating used.

From in vitro and in vivo studies it has become evident that both Herp1 and Herp2 act downstream of the Notch/ICD (IntraCellular Domain) signaling ${ }^{35,36}$ and regulate SMC differentiation, migration and stimulate vascular remodeling in response to trauma $^{3-7}$. In a previous study the Notch1-Herp1 axis was reported to stimulate vascular SMC proliferation and migration in vitro and neointimal formation in rodents after vascular injury ${ }^{3,4,25}$. In accordance, we observed up-regulation of Herp2 expression in the media and neointima of injured vessels, which was reduced in injured vessels treated with sJag1. In vitro, Ad-sJag1 infection or recombinant sJag1 stimulation reduced Herp2 mRNA expression in SMCs, suggesting an inhibitory effect on Notch signaling. The observation that the hampered migration and proliferation of the sJag treated SMCs could be partially rescued by Herp2 overexpression, suggests that Notch-Herp2 signaling is required for SMC function and that sJag is inhibitory. Considering the overlap in expression and function between Herp1 and Herp2 in cardiovascular development and SMC function, together with our data, it can be concluded that Herp2 plays a role in neointima formation as well.

Notch signaling has been reported to promote SMC differentiation ${ }^{23,26}$. Recently, Liu et $\mathrm{al}^{5}$ demonstrated that Jag1-induced Notch3 signaling initiates an autoregulatory loop that results in Notch3 overexpression and simultaneously enhanced the expression of SMC markers such as $\alpha$ SMA, CALPONIN and SM-MHC. We also showed that Notch3 expression in carotid artery sections in the Ad-sJag1 group was reduced concomitant with the SMC marker $\alpha \mathrm{SMA}$ expression at day 3 , confirming the positive role of Notch in regulating SMC differentiation. In contrast, other studies have shown that NOTCH can negatively affect SMC differentiation by interfering with SRF (Serum response factor) and myocardin ${ }^{7,25}$, that are both essential for the expression of SMC markers. The apparent controversy in data likely relates to the balance between NICD/RBPkJ ${ }^{7}$ and Notch target genes (Herp1 and 2) ${ }^{37}$ that can induce and inhibit SMC differentiation, respectively.

In conclusion, we report that sJag1 inhibits neointima formation in a rat carotid artery injury model by interfering with Notch-Herp2 signaling. The sJag1 inhibitory effect on Notch activation is likely related to its interference with membrane bound 
ligand induced signaling. Notch ligands induce signaling only when expressed on the cell surface or immobilized to a substrate ${ }^{31,37}$, whereas they show none or antagonistic activity when expressed as soluble forms ${ }^{31,37,38}$. Here, we describe an interesting approach to reduce neointima formation by blocking Notch signaling with sJag1, providing a potentially applicable clinical tool for lowering the risk of restenosis in patients with peripheral artery disease.

\section{Acknowledgments}

Technical support of Drs Nynke van den Akker and Herman Popeijus and Niek van Stipdonk is highly appreciated. This manuscript is dedicated to the late Thomas Maciag who initiated this collaborative research. This research was supported by The Netherlands Heart Foundation (grant 2005B254 to M.P.) and The Marie Curie FP6 early stage researcher training (MEST-CT-2005-020706 to CARIM). No disclosures. 


\section{References}

1. Owens GK, Kumar MS, Wamhoff BR. Molecular regulation of vascular smooth muscle cell differentiation in development and disease. Physiol Rev. 2004;84:767-801.

2. Rensen SS, Doevendans PA, van Eys GJ. Regulation and characteristics of vascular smooth muscle cell phenotypic diversity. Neth Heart J. 2007;15:100-108.

3. Doi H, Iso T, Shiba $Y$, Sato H, Yamazaki M, Oyama $Y$, Akiyama H, Tanaka T, Tomita T, Arai M, Takahashi $\mathrm{M}$, Ikeda $\mathrm{U}$, Kurabayashi $\mathrm{M}$. Notch signaling regulates the differentiation of bone marrowderived cells into smooth muscle-like cells during arterial lesion formation. Biochem Biophys Res Commun. 2009;381:654-659.

4. Li Y, Takeshita K, Liu PY, Satoh M, Oyama N, Mukai Y, Chin MT, Krebs L, Kotlikoff MI, Radtke F, Gridley T, Liao JK. Smooth muscle Notch1 mediates neointimal formation after vascular injury. Circulation. 2009;119:2686-2692.

5. Liu H, Kennard S, Lilly B. NOTCH3 expression is induced in mural cells through an autoregulatory loop that requires endothelial-expressed JAGGED1. Circ Res. 2009;104:466-475.

6. Sweeney C, Morrow D, Birney YA, Coyle S, Hennessy C, Scheller A, Cummins PM, Walls D, Redmond EM, Cahill PA. Notch 1 and 3 receptor signaling modulates vascular smooth muscle cell growth, apoptosis, and migration via a CBF-1/RBP-Jk dependent pathway. Faseb J. 2004;18:1421-1423.

7. Tang Y, Urs S, Liaw L. Hairy-related transcription factors inhibit Notch-induced smooth muscle alphaactin expression by interfering with Notch intracellular domain/CBF-1 complex interaction with the CBF-1-binding site. Circ Res. 2008;102:661-668.

8. Artavanis-Tsakonas $\mathrm{S}$, Rand MD, Lake RJ. Notch signaling: cell fate control and signal integration in development. Science. 1999;284:770-776.

9. Roca C, Adams RH. Regulation of vascular morphogenesis by Notch signaling. Genes Dev. 2007;21:2511-2524.

10. Gridley T. Notch signaling in vascular development and physiology. Development. 2007;134:27092718.

11. Bray SJ. Notch signalling: a simple pathway becomes complex. Nat Rev Mol Cell Biol. 2006;7:678689.

12. Iso T, Kedes L, Hamamori Y. HES and HERP families: multiple effectors of the Notch signaling pathway. J Cell Physiol. 2003;194:237-255.

13. Ehebauer M, Hayward P, Martinez-Arias A. Notch signaling pathway. Sci STKE. 2006;2006:cm7.

14. LaVoie MJ, Selkoe DJ. The Notch ligands, Jagged and Delta, are sequentially processed by alphasecretase and presenilin/gamma-secretase and release signaling fragments. I Biol Chem. 2003;278:34427-34437.

15. Kolev V, Kacer D, Trifonova R, Small D, Duarte M, Soldi R, Graziani I, Sideleva O, Larman B, Maciag T, Prudovsky I. The intracellular domain of Notch ligand Delta1 induces cell growth arrest. FEBS Lett. 2005;579:5798-5802.

16. Lindner V, Booth C, Prudovsky I, Small D, Maciag T, Liaw L. Members of the Jagged/Notch gene families are expressed in injured arteries and regulate cell phenotype via alterations in cell matrix and cell-cell interaction. Am J Pathol. 2001;159:875-883.

17. Morrow D, Guha S, Sweeney C, Birney Y, Walshe T, O'Brien C, Walls D, Redmond EM, Cahill PA. Notch and vascular smooth muscle cell phenotype. Circ Res. 2008;103:1370-1382.

18. Krebs LT, Xue Y, Norton CR, Shutter JR, Maguire M, Sundberg JP, Gallahan D, Closson V, Kitajewski J, Callahan R, Smith GH, Stark KL, Gridley T. Notch signaling is essential for vascular morphogenesis in mice. Genes Dev. 2000;14:1343-1352.

19. Xue Y, Gao X, Lindsell CE, Norton CR, Chang B, Hicks C, Gendron-Maguire M, Rand EB, Weinmaster G, Gridley T. Embryonic lethality and vascular defects in mice lacking the Notch ligand Jagged1. Hum Mol Genet. 1999;8:723-730.

20. Campos AH, Wang W, Pollman MJ, Gibbons GH. Determinants of Notch-3 receptor expression and signaling in vascular smooth muscle cells: implications in cell-cycle regulation. Circ Res. 2002;91:9991006. 
21. Domenga V, Fardoux P, Lacombe P, Monet M, Maciazek J, Krebs LT, Klonjkowski B, Berrou E, Mericskay M, Li Z, Tournier-Lasserve E, Gridley T, Joutel A. Notch3 is required for arterial identity and maturation of vascular smooth muscle cells. Genes Dev. 2004;18:2730-2735.

22. Chabriat H, Joutel A, Dichgans M, Tournier-Lasserve E, Bousser MG. Cadasil. Lancet Neurol. 2009;8:643-653.

23. Doi H, Iso T, Sato H, Yamazaki M, Matsui H, Tanaka T, Manabe I, Arai M, Nagai R, Kurabayashi M. Jagged1-selective notch signaling induces smooth muscle differentiation via a RBP-Jkappadependent pathway. J Biol Chem. 2006;281:28555-28564.

24. Wang W, Prince CZ, Hu X, Pollman MJ. HRT1 modulates vascular smooth muscle cell proliferation and apoptosis. Biochem Biophys Res Commun. 2003;308:596-601.

25. Doi H, Iso T, Yamazaki M, Akiyama H, Kanai H, Sato H, Kawai-Kowase K, Tanaka T, Maeno T, Okamoto E, Arai M, Kedes L, Kurabayashi M. HERP1 inhibits myocardin-induced vascular smooth muscle cell differentiation by interfering with SRF binding to CArG box. Arterioscler Thromb Vasc Biol. 2005;25:2328-2334.

26. Noseda M, Fu Y, Niessen K, Wong F, Chang L, McLean G, Karsan A. Smooth Muscle alpha-actin is a direct target of Notch/CSL. Circ Res. 2006;98:1468-1470.

27. Wang W, Campos AH, Prince CZ, Mou Y, Pollman MJ. Coordinate Notch3-hairy-related transcription factor pathway regulation in response to arterial injury. Mediator role of platelet-derived growth factor and ERK. J Biol Chem. 2002;277:23165-23171.

28. Lindner V. Expression of platelet-derived growth factor ligands and receptors by rat aortic endothelium in vivo. Pathobiology. 1995;63:257-264.

29. Lindner V, Reidy MA. Platelet-derived growth factor ligand and receptor expression by large vessel endothelium in vivo. Am J Pathol. 1995;146:1488-1497.

30. Sakata Y, Xiang F, Chen Z, Kiriyama Y, Kamei CN, Simon DI, Chin MT. Transcription factor CHF1/Hey2 regulates neointimal formation in vivo and vascular smooth muscle proliferation and migration in vitro. Arterioscler Thromb Vasc Biol. 2004;24:2069-2074.

31. Small D, Kovalenko D, Kacer D, Liaw L, Landriscina M, Di Serio C, Prudovsky I, Maciag T. Soluble Jagged 1 represses the function of its transmembrane form to induce the formation of the Srcdependent chord-like phenotype. J Biol Chem. 2001;276:32022-32030.

32. Duarte A, Hirashima M, Benedito R, Trindade A, Diniz P, Bekman E, Costa L, Henrique D, Rossant J. Dosage-sensitive requirement for mouse Dll4 in artery development. Genes Dev. 2004;18:24742478.

33. Trindade A, Kumar SR, Scehnet JS, Lopes-da-Costa L, Becker J, Jiang W, Liu R, Gill PS, Duarte A. Overexpression of delta-like 4 induces arterialization and attenuates vessel formation in developing mouse embryos. Blood. 2008;112:1720-1729.

34. Caolo V, van den Akker NM, Verbruggen S, Donners MM, Swennen G, Schulten H, Waltenberger J, Post MJ, Molin DG. Feed-forward signaling by membrane-bound ligand receptor circuit: the case of NOTCH DELTA-like 4 ligand in endothelial cells. J Biol Chem. 2010;285:40681-40689.

35. Iso T, Chung G, Hamamori Y, Kedes L. HERP1 is a cell type-specific primary target of Notch. $J$ Biol Chem. 2002;277:6598-6607.

36. Jarriault S, Brou C, Logeat F, Schroeter EH, Kopan R, Israel A. Signalling downstream of activated mammalian Notch. Nature. 1995;377:355-358.

37. Varnum-Finney B, Wu L, Yu M, Brashem-Stein C, Staats S, Flowers D, Griffin JD, Bernstein ID. Immobilization of Notch ligand, Delta-1, is required for induction of notch signaling. J Cell Sci. 2000;113 Pt 23:4313-4318.

38. Hicks C, Ladi E, Lindsell C, Hsieh JJ, Hayward SD, Collazo A, Weinmaster G. A secreted Delta1-Fc fusion protein functions both as an activator and inhibitor of Notch1 signaling. J Neurosci Res. 2002;68:655-667. 


\section{Supplement Material}

\section{Methods}

Balloon angioplasty and adenovirus infection of rat carotid arteries

All animal studies were approved by the Animal Welfare Committee of the University of Maastricht. Animals were handled in accordance with the American Physiological Society guidelines for animal welfare and the Guide for Care and Use of Laboratory Animals published by the United States National Institutes of Health. Sprague Dawley rats $(n=48)$ were anesthetized with $80 \mathrm{mg} / \mathrm{kg}$ of ketamine (Fort Dodge Animal Health, Fort Dodge, IA) and $8 \mathrm{mg} / \mathrm{kg}$ of xylazine (Phoenix Pharmaceutical, St. Joseph, MO, US) and for pain medication Temgesic (SheringPlough, Houten, the Netherlands) was applied $(0.01 \mathrm{mg} / \mathrm{kg} 30$ minutes preoperative and 3 times within 24 hours post-operative) all i.p. Vascular injury was performed as described by Clowes ${ }^{1}$. Next the common carotid artery was instilled with $100 \mu \mathrm{L} 5.0 \times 10^{11} \mathrm{VP} / \mathrm{mL}$ Adenoviral LacZ (Ad-LacZ), adenoviral full length Jagged1 (Ad-FI-Jag1) or adenoviral soluble Jagged1 (Ad-sol-Jag1) for 10 minutes.

\section{Immunohistochemistry}

Carotid artery tissue of non-treated and day 3 and 14 post denudation (in total $\mathrm{n}=25$ ) was paraffin imbedded and cross sectioned at $5 \mu \mathrm{m}$, deparaffinated, rehydrated and subjected to antigen retrieval by heating the sections (12 minutes to $98^{\circ} \mathrm{C}$ ) in citric acid buffer ( $\left.0.01 \mathrm{Mol} / \mathrm{L}, \mathrm{pH} 6.0\right)$. Endogenous peroxidase activity was blocked by incubating the sections for 20 minutes in PBS, $0.3 \% \mathrm{H}_{2} \mathrm{O}_{2}$ before adding the primary antibody for Jagged-1 (sc-6011), Notch-1 (sc-6014R), Notch-3 (sc-7424) (all Santa Cruz Biotechnolgy, US) or Herp2, which was kindly provided by Dr Larry Kedes. For aSMA staining sections were incubated with A2547 Ab (Sigma-Adrich, Missouri, US) as previously reported by Skalli et al $^{2}$ and for PCNA staining sections were incubated with the PCNA PC10 Ab (Roche Diagnostics, Indianapolis, US).

Subsequently each section was incubated for 1 hour with peroxidase-labelled antibody (rabbit- $\alpha$-mouse; P0260, DAKO, Glostrup, Denmark) or biotin-labelled secondary antibody (goat- $\alpha$-rabbit (BA-1000), horse- $\alpha$-mouse (BA-2000) and horse$\alpha$-goat (BA-9500), all Vector Labs, Burlingame, US). When using biotin labelled antibody, additional incubation with the Vectastain $A B C$ staining kit (PK-6100, Vector Labs, Burlingame, US) was performed for 45 minutes. The sections were mounted with Entellan (Merck, Darmstadt, Germany). Visualization was performed with the DAB procedure and Mayer's hematoxilin was used as a counterstaining. 
Adenoviral transduction and treatment with recombinant Jag1 or DLL4 proteins. Human umbilical vein endothelial cells (HUVECS), human coronary arterial endothelial cells (HCAEC) and human coronary artery smooth muscle cells (CASMCs) (all from Lonza, Verviers, Belgium) were transduced with $5 \times 10^{9} \mathrm{VP} / \mathrm{mL}$ Ad-LacZ, AdsJag1 (c-myc tagged) ${ }^{3}$ or Ad-Fl-Jag1. For stimulation experiments with attached ligand rDll4 or rJag1, ECs were grown for 48 hours on $0.2 \%$ gelatin coated plates containing $1 \mu \mathrm{g} / \mathrm{mL}$ recombinant rDLL4 or Jag1 (R\&D System; Abingdon, Oxfordshire, UK) as previously described by Williams et al $^{4}$. Stimulation of CASMCs with soluble Jag1 was performed by adding $5 \mu \mathrm{g} / \mathrm{mL}$ of recombinant sJag1 (R\&D System) to the medium. ECs and CASMCs, infected or treated with recombinant protein, were cultured in EGM-2 and SmGM-2 medium (Lonza), respectively at $37{ }^{\circ} \mathrm{C}$ with $5 \% \mathrm{CO}_{2}$. Forty eight hours after infection cells transduced with Ad-sJag1 were treated with Brefeldin A (10 ng/mL, Sigma, St. Louis, MO, US) for 4 hours. Cells were lysed with RIPA buffer (50 mM HEPES pH 7.4, $150 \mathrm{mM} \mathrm{NaCl} 5 \mathrm{mM}$ EGTA, $5 \mathrm{mM}$ EDTA, 50 mM NaF, 20 mM NaPyrophosphate, 1 \% Triton X-100, 1 mM PMSF, 1 mM $\mathrm{Na}_{3} \mathrm{VO}_{4}$ ). Immunoprecipitates were separated on a $10 \%$ SDS-polyacrylamide gel and transferred to an Immobilon-P PVDF membrane (Millipore, Billerica, MA, US). Membranes were stained with anti-c-Myc or anti-Jagged1 antibody as described previously.

\section{Infection efficiency}

The infection efficiency in vivo was determined by performing a $\beta$-galactosidase enzymatic staining at 3 day post injury in carotid artery sections that have been instilled with $100 \mu \mathrm{L} 5.0 \times 10^{11} \mathrm{VP} / \mathrm{mL}$. The infection efficiency was also assessed by immunofluorescent staining of cultured cells upon Ad-FI-Jag1 infection. CASMCs were cultured on 4 wells slides (BD, Franklin Lakes, USA) until confluence and infected with Ad-Fl-Jag1 or Ad-LacZ. After 24 hours cells were incubated for 1 hour with the primary goat polyclonal anti-Jagged1 (Santa Cruz sc-6011). Slides were washed and incubated with $2 \%$ rabbit serum and secondary Ab Alexa-Fluo594 Rabbit anti-Goat A-11080 (Invitrogen). Photos were taken using a Leica microscope with DFC350 FX digital camera.

\section{Proliferation and migration assays in HUVECS or CASMCS}

To assess HUVECs or CASMCs proliferation CellTiter $96^{\circ}$ AQueous One solution Cell Proliferation Assay (Promega, San Luis Obispo, CA, US) was used. Per well $2 \times 10^{3}$ cells were plated in a 96 well tissue culture plate for 48 hours after adenoviral transduction and were incubated overnight in appropriate growth medium. Cell Proliferation Assay was added directly to culture wells, incubating for 2 hours and then recording absorbance at $490 \mathrm{~nm}$ on a Victor-3 fluorescent microplate reader (Perkin Elmer).The same protocol was used to determine migration of CASMCs previously transfected with pcDNA3.2-Hey1 (Herp2) or mock and incubated with or 
without $5 \mu \mathrm{g} / \mathrm{mL}$ recombinant Jagged1 (R\&D technologies). Proliferation of HUVECs seeded on fibronectin coating 96 well tissue culture plate after Ad-sJag1 transduction was also assessed. Proliferation was defined as the average of four independent wells.

For the migration wounding assay, HUVECs were grown to confluence in 6-well plates and the monolayer was scratched with a pipette tip. Gap width was measured at marked locations from images taken by inverted microscope (TMS-F, Nikon), immediately after the scratching and 10 hours later. Relative migratory distances were calculated. Independent experiments were performed 3 times. Migration of HUVECs cultured on fibronectin was assessed by performing an Oris $^{\mathrm{TM}}$ Cell Migration Assay (Platypus Technologies, LLC, USA). Per well $1.3 \times 10^{4}$ cells were seeded on 96 wells plate coated or not with fibronectin. The next day cells were infected with Ad-LacZ or Ad-sJag1. After 24 hours cells were stained according to protocol instructions and migration was assessed by measuring fluorescence on a Victor-3 fluorescent microplate reader (Perkin Elmer, Groningen, the Netherlands) in bottom read mode. For transmigration assays, CASMC were labeled with calceinAM $\left(5 \mu \mathrm{L} / \mathrm{mL}\right.$, Molecular Probes, Eugene, OR) and $5 \times 10^{4}$ cells were placed in the FALCON HTS FluoroBlock transwell system inserts (pore size $8 \mu \mathrm{m}$; Becton Dickinson, Bedford, MA) in DMEM supplemented with $0.5 \%$ fetal bovine serum (Invitrogen, Carlsbad, California) and incubated for 8 hours at $37{ }^{\circ} \mathrm{C}$. The lower chamber of the FluoroBlock contained DMEM supplemented with PDGF-BB (10 $\mathrm{ng} / \mathrm{mL}$ ) and $0.5 \%$ fetal bovine serum. The extent of cell migration was estimated by measuring fluorescence on a Victor-3 fluorescent microplate reader (Perkin Elmer, Groningen, the Netherlands) in bottom read mode. The same protocol was employed to determine migration of CASMCs that were transfected with pcDNA3.2Hey1 (Herp2) or mock and were incubated with or without $5 \mu \mathrm{g} / \mathrm{mL}$ recombinant Jagged1 (R\&D technologies). Three independent experiments were perfomed.

SMC proliferation in vivo was assed by counting the number of PCNA positive nuclei in a given area of the vessel of day 3 and 14 Ad-LacZ and Ad-sJag1 treated balloon injured carotid arteries ( $n=6$ per group).

\section{RNA expression analysis}

Twenty four hours after infection or treatment with recombinant sJag1 protein, RNA was isolated by using RNeasy micro-kit Qiagen (Qiagen, $\mathrm{GmbH}$, Hilden, Germany). A total of 100 ng RNA per sample was subjected to reverse transcription (RT). qPCR was performed using Superscript ${ }^{\mathrm{TM}}$ III Platinum Two-step qRT-PCR kit with SYBR green (Invitrogen) and primer concentration of $10 \mu \mathrm{M}$ according to van den Akker et $\mathrm{al}^{6}$. PCR Primers used were: human $\beta$-ACTIN-sense: 5'-ATCCTCACCCTGAAGTACCC-3', $\beta$-ACTIN-antisense: 5'-CACGCAGCTCATTGTAGAAG-3'; GADPH-sense: 5' GCCTCAAGATCATCAGCAAT-3'; GADPH-antisense: 5'-GGACTGTGGTCATGAGTCCT-3'; HES1-sense: 5'-CCAAAGACAGCATCTGAGCA-3', HES1-antisense: 5' 
GCCGCGAGCTATCTTTCTT-3'; HERP1-sense: 5'-GAAGATGCTTCAGGCAACAG-3' HERP1-antisense: 5'-GCAACTTCTGTTAGGCACTCTC-3'; HERP2-sense: 5' CGCCCTTGCTATGGACTATC-3', HERP2-antisense: 5'-TGCGAAACCAGTCGAACTC-3'; HERP3-sense: 5'-CGCATCAACAGTAGCCTTTCT-3', HERP3-antisense: 5'GGCATGGAGCATTTTCAAGT-3'. Primers were designed with oligoperfect ${ }^{\mathrm{TM}}$ ' Designer $^{\prime}$ (Invitrogen), Primer3 and Mfold (http: // www.idtdna.com/ scitools/ Application/ $\mathrm{mfold} /$ ) and were synthesized by Eurogentec (Seraing, Belgium). qPCR reactions were run on a BioRad MiQ Real Time PCR detection System (BioRad, Veenendaal, the Netherlands).

\section{Carotid artery perfusions and silver staining}

Two weeks after carotid artery injury upper body perfusion was performed through cannulation of the ascending aorta. In brief, after rinsing with heparinized saline, the upper circulation was perfused with fixative (1\% paraformaldehyde (PFA) and $0.5 \%$ glutaraldehyde in $75 \mathrm{mmol} / \mathrm{L}$ cacodylate, $\mathrm{pH} 7.4$ ), followed by saline and $5 \%$ glucose and $0.1 \%$ silver nitrate under $90 \mathrm{~mm} \mathrm{Hg}$ pressure. Thereafter carotid arteries were removed and post fixed, opened longitudinally, exposed to light, and dehydrated and mounted in Permaslip mounting medium (Alban Scientific Inc, St Louis, MO, US). Endothelial regeneration was assessed using an Olympus BH-2 microscope with a Sony SSC C374 camera and NIH Image- ${ }^{\circledR}$ software. Endothelial covering was calculated by two independent investigators blinded to the experimental groups (Inter-observer variability was <2 \%) as the percentage regenerating endothelial cells divided by the total area.

\section{Morphometric analysis}

Carotid artery segments of $5 \mathrm{~mm}$ were fixed in $4 \%$ PFA for 30 minutes and imbedded in Tissue-Tek OCT media (Sakura Finetek USA, Inc. Torrance, CA, US). Sections of $5 \mu \mathrm{m}$ were stained with Elastin van Gieson, hematoxylin and eosin (H\&E). From each section, luminal area, inside the internal elastic lamina, and the area encircled by the external elastic lamina were traced manually and measured with standard computer assisted planimetry in a double blinded manner. Medial area was calculated as area encircled by external elastic lamina minus area inside the internal elastic lamina. Intimal area was calculated as area inside the internal elastic lamina minus luminal area. From these calculations the ratio of intimal to medial area was defined. 


\section{Supplemental Figures}
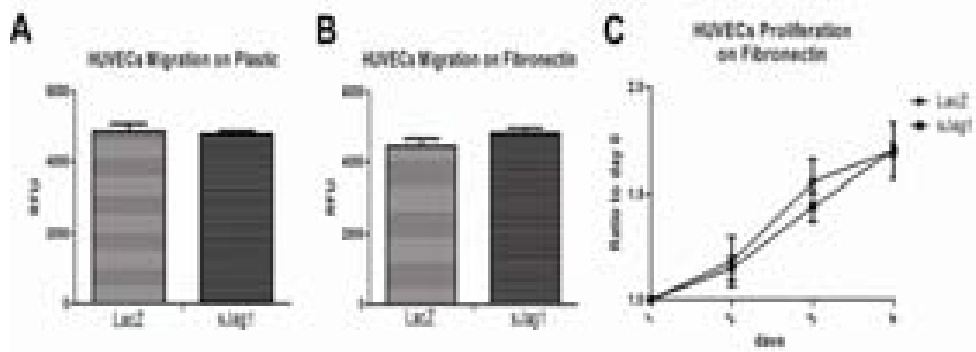

Figure I Migration and proliferation assay on HUVECs infected with Ad-sJag1 (sJag1) or Ad-LacZ (LacZ) and cultured on plastic or fibronectin. A) No difference in migration rate was observed in HUVECS infected with Ad-LacZ or Ad-sJag1 when cultured on plastic. B) Likewise, Ad-sJag1 had no effect on HUVECs cultured on a fibronectin coated surface. C) Proliferation rate of HUVECs transduced with AdLacZ or Ad-sJag1 and cultured on fibronectin was comparable. Data represent mean \pm SD of three independent experiments.

A

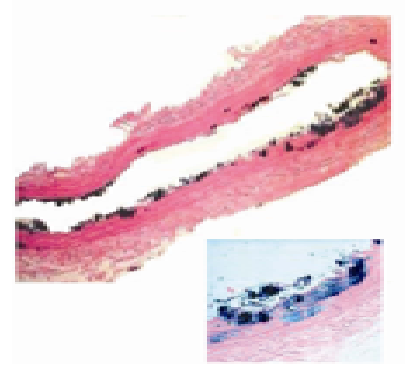

B

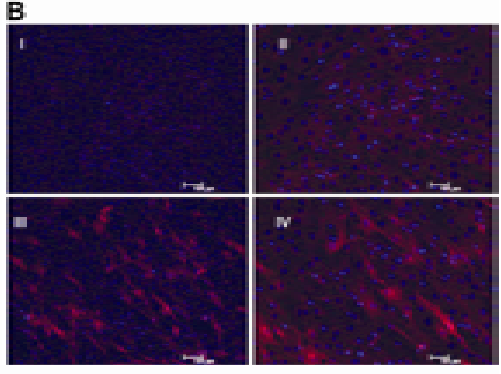

Figure II $\beta$-galactosidase and Jagged1 staining showing the infection efficiency in vivo and in vitro, respectively. A) $\beta$-Galactosidase expressed as blue cells at day 3 post-injury in the rat carotid artery that had been instilled with $5 \times 10^{11} \mathrm{VP} / \mathrm{mL}$ Ad-LacZ for 10 minutes. X-gal staining showed blue stained cells in the transfected artery. B) Immunofluorescence analysis of CASMCs infected with Ad-LacZ or Ad-Fl-Jag1 5 $\mathrm{x} 10^{9} \mathrm{VP} / \mathrm{mL}$ for 24 hours for Jagged-1. CASMCs infected with Ad-Fl-Jag1 showed higher Jagged1 staining (red) (panels III and IV, 10X and 20X magnification, respectively) as compared with Ad-LacZ infected cells (panel I and II, 10X and 20X magnification, respectively) showing only the expression of endogenous Jagged1. 


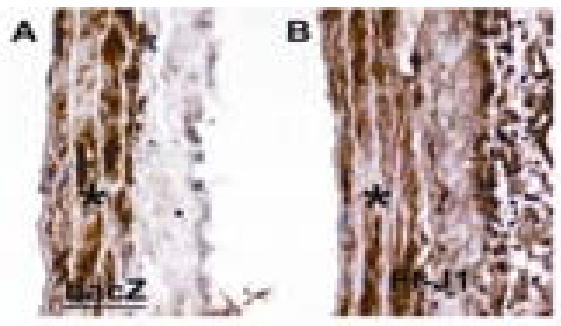

Figure III Cross sections of balloon injured and Ad-LacZ (A), Ad-Fl-Jag1 (B) transduced carotid arteries showing Jag1 expression at 3 days post injury. A higher Jag1 signal was apparent for the Ad-Fl-Jag1 section as compared with the Ad-LacZ control. Asterisk represents the media. Scale bar: $200 \mu \mathrm{m}$.
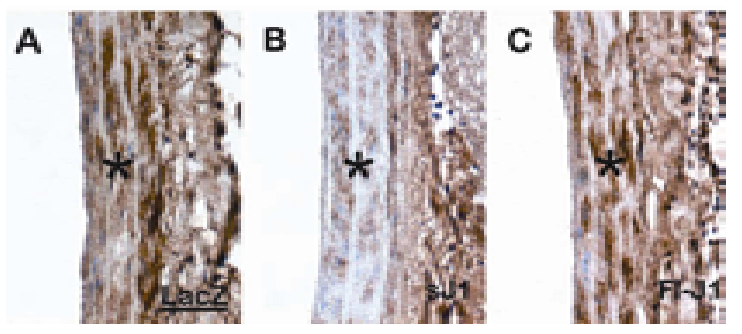

Figure IV Cross sections of balloon injured and Ad-LacZ (A), Ad-sJag1 (B) and Ad-FI-Jag1 (C) transduced carotid arteries showing Notch3 expression at 3 days post injury. Notch3 expression was lower in AdsJag1 (B) than in Ad-LacZ control (A) or Ad-Fl-Jag1 (C) groups. Asterisk represents the media. Scale bar: $200 \mu \mathrm{m}$.

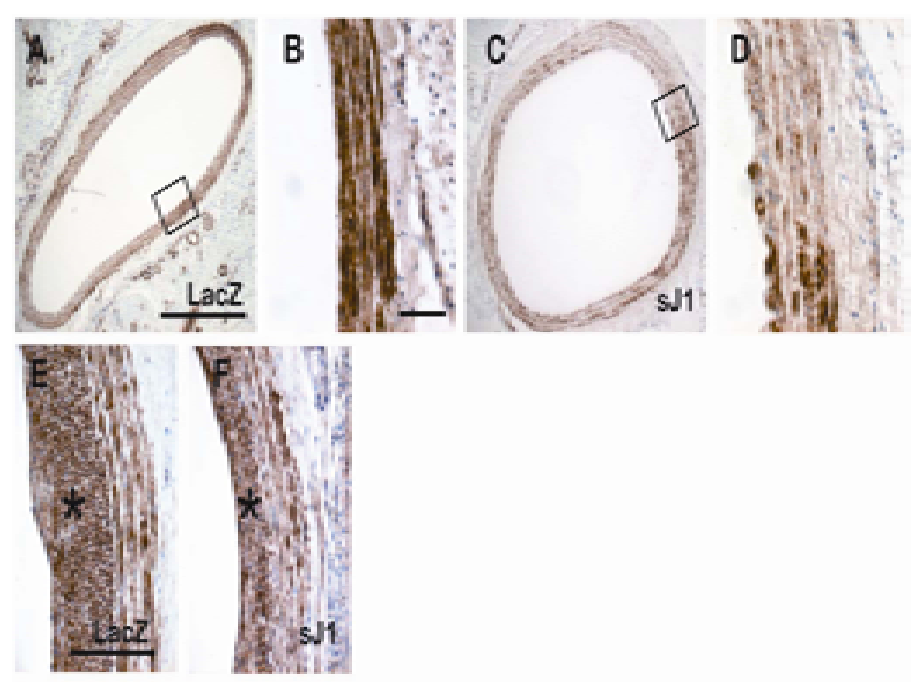

Figure $\mathbf{V}$ Cross sections of balloon injured and Ad-LacZ ( $A, B$ and $E$ ) and Ad-sJag1 (C, D, F) transduced carotid arteries showing alpha-smooth muscle actin ( $\alpha \mathrm{SMA}$ ) expression at 3 and 14 days post injury. aSMA was expressed at a lower level in the media of Ad-sJag1 (C) infected arteries as compared to AdLacZ controls (A) at 3 days post-injury. (B) and (D) are higher magnifications of the boxed area in (A) and 
(C), respectively. (E) At 14 days following vascular injury, the SMCs within the neointima strongly express aSMA at a level comparable to the SMCs of the media. (F) Ad-sJag1 transduced carotid arteries showing comparable $\alpha S M A$ level in the media and neointima as the control Ad-LacZ (E). Asterisk represents the neointima. Scale bar: A and C $800 \mu \mathrm{m}$; B and D $150 \mu \mathrm{m}$; and E-F $350 \mu \mathrm{m}$.

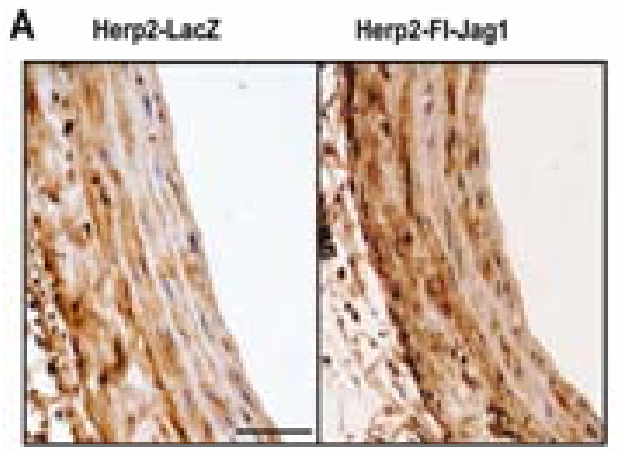

B

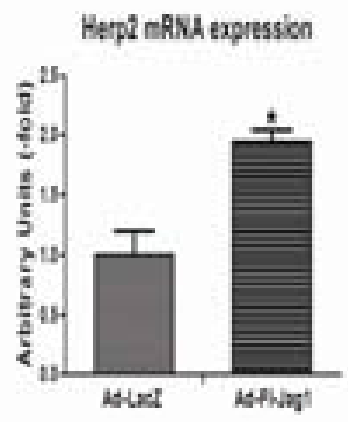

Figure VI Cross sections of balloon injured and Ad-LacZ or Ad-Fl-Jag1 transduced carotid arteries showing Herp2 expression at 3 days post-injury (A) and HERP2 mRNA expression in CASMCs infected with Ad-LacZ or Ad-Fl-Jag1 (B). Ad-Fl-Jag treated arteries (A, right panel) showed a comparable medial Herp2 expression to Ad-LacZ treated arteries (A, left panel). HERP2 mRNA expression was significantly increased in Ad-Fl-Jag1 transfected CASMCs as compared to Ad-LacZ infected cells (B). Data are presented as mean \pm SD of three independent experiments. Scale bare is $200 \mu \mathrm{m}$.

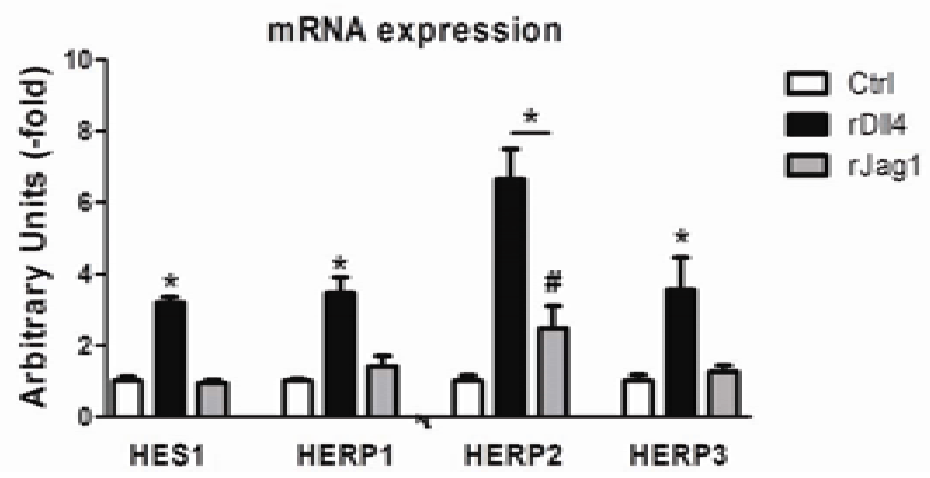

Figure VII mRNA expression of Notch target genes of ECs stimulated with immobilized rDLL4 or rJag1. rDLL4 stimulated ECs showed a significant $\left({ }^{*}\right)$ increase in HES1, HERP1, 2 and 3 mRNA expression (rDLL4, black bars) compared to non-stimulated cells (Ctrl, open bars). Cells cultured on rJag1 showed only an increase in HERP2 expression (rJag1, grey bars) that was significantly less (\#) than the increase induced by rDLL4. Data presented as mean \pm SE of three independent experiments. 


\section{References}

1. Clowes AW, Clowes MM, Fingerle J, Reidy MA. Kinetics of cellular proliferation after arterial injury. V. Role of acute distension in the induction of smooth muscle proliferation. Lab Invest. 1989;60:360364.

2. Skalli O, Ropraz P, Trzeciak A, Benzonana G, Gillessen D, Gabbiani G. A monoclonal antibody against alpha-smooth muscle actin: a new probe for smooth muscle differentiation. J Cell Biol. 1986;103:2787-2796.

3. Small D, Kovalenko D, Kacer D, Liaw L, Landriscina M, Di Serio C, Prudovsky I, Maciag T. Soluble Jagged 1 represses the function of its transmembrane form to induce the formation of the Srcdependent chord-like phenotype. J Biol Chem. 2001;276:32022-32030.

4. Williams CK, Li JL, Murga M, Harris AL, Tosato G. Up-regulation of the Notch ligand Delta-like 4 inhibits VEGF-induced endothelial cell function. Blood. 2006;107:931-939.

5. Horowitz A, Simons M. Phosphorylation of the cytoplasmic tail of syndecan-4 regulates activation of protein kinase Calpha. J Biol Chem. 1998;273:25548-25551.

6. van den Akker NM, Caolo V, Wisse LJ, Peters PP, Poelmann RE, Carmeliet P, Molin DG, Gittenbergerde Groot AC. Developmental coronary maturation is disturbed by aberrant cardiac vascular endothelial growth factor expression and Notch signalling. Cardiovasc Res. 2008;78:366- 375. 



\title{
Chapter 6 \\ General Discussion
}

\author{
New insights in the NOTCH Signaling Pathway \\ VEGF induced DLL4 is mediated by VEGFR-2 and NOTCH signaling \\ A positive feed-forward loop is responsible for the VEGF-A induced DLL4 expression \\ NOTCH-induced DLL4 expression: feed-forward or feed-back signaling? \\ VEGF induced NOTCH signaling and DLL4 overexpression by activating ADAM-10
}

\section{Consequence of altered NOTCH signaling at Tissue/Organ Level}

DLL4 blockade: potential therapeutic implication for inhibition of tumour growth VEGF and NOTCH in coronary artery development: effects on endothelial differentiation and development of the media

Inhibition of NOTCH-signaling by soluble Jagged1: a new tool to inhibit neointima formation

\section{Conclusion}




\section{New insights in the NOTCH Signaling Pathway}

The role of NOTCH in cardiovascular development and its implication in regulating physiological and pathophysiological vascular biology has received particular attention over the last years ${ }^{1-10}$. In this thesis the role of the NOTCH signaling pathways in vascular biology has been investigated with special focus on the effects of VEGF on DLL4 expression, NOTCH signaling and ADAM activity in endothelial cells (ECs), and the consequence of altered NOTCH signaling for vessel function.

\section{VEGF induced DLL4 is mediated by VEGFR-2 and NOTCH signaling}

DLL4-induced NOTCH signaling is required for endothelial sprouting and branching of blood vessels. Among endothelial cell (EC), the ones that are highly VEGFR-2 positive will localize at the sprouting edge and will develop into endothelial tip cells. In response to VEGF-A, these cells will start sprouting by elongating filopodia, and will upregulate DLL4 expression. Subsequently, interaction of the DLL4 positive tip cells with adjacent ECs triggers NOTCH signaling in the latter and, eventually, differentiation into a stalker cell ${ }^{11}$. The stalker cell exhibits low migration and filopodia capacity ${ }^{1,2,5,11-14}$ and will become the endothelium lining the lumen of the newly branched vessel structure. The VEGF signaling appears self-limiting as DLL4induced NOTCH signaling in turn triggers the downregulation of VEGFR-2 ${ }^{6,8}$. VEGFsignaling is further silenced by enhancing the expression of VEGFR-1 and soluble VEGFR-1 (sVEGFR-1) that both are considered negative regulators of VEGF signaling in ECs by sequestration of VEGF ${ }^{15,16}$. VEGFR-2 shows a stronger tyrosine kinase activity than VEGFR-1 and is regarded the main receptor to mediate VEGF signaling in vascular ECs ${ }^{17-19}$. VEGFR-2 has therefore been considered the major player in facilitating the VEGF-A induced DLL4 expression ${ }^{6,8}$, although no direct evidence was provided that fully justified this assumption. We established that the expression of DLL4 in ECS is specifically stimulated by VEGFR-2 and not by VEGFR-1 mediated signaling (Chapter 2). Intriguingly, VEGF-A165-induced DLL4 expression was prevented by NOTCH signaling inhibitors, indicating that NOTCH signaling itself is required for increased DLL4 expression downstream of VEGF/VEGFR-2. These observations strongly suggest that VEGF induces DLL4 expression in a manner that is dependent on VEGF-induced NOTCH signaling.

A positive feed-forward loop is responsible for the VEGF-A induced DLL4 expression In addition to the VEGF-A induced NOTCH activation, the FOXC forkhead transcription factors have also been implicated to mediate DLL4 expression downstream of VEGF/VEGFR-2 and independent of NOTCH activation. Although Seo et $\mathrm{al}^{20}$ have shown that FOXC1 and more efficiently FOXC2 transactivated the DLL4 promoter in a dose dependent manner, mutation of the Forkhead Binding Element (FBE) did not attenuate the induction of DLL4. The authors concluded that a 
combinatorial regulation by different classes of transcription factors in addition to FOXC could be responsible for the regulation of DLL4 transcription. We found that inhibition of NOTCH signaling perturbed VEGF-induced DLL4 expression, suggesting a direct role for $\mathrm{NOTCH}$ in this process. Furthermore, we showed that increased NOTCH signaling by overexpression of NICD-4 and NICD-1 effectively transactivated deletional fragments of the DLL4 promoter that contained several RBP-Jk binding sites, but lacked the $\mathrm{FBE}^{21}$ (Chapter 2). Moreover, overexpressing a constitutively active FOXC2 construct in endothelial cells was without clear effect on DLL4 expression (Chapter 2). Hayashi and $\mathrm{Kume}^{22}$ showed that FOXC2 and more effectively NICD were individually able to transactivate the promoter of the NOTCH target gene Hey2. However in combination they act synergistically inducing a more potent transactivation of the promoter. We can not exclude that a similar mechanism could affect DLL4 expression as well.

\section{NOTCH-induced DLL4 expression: feed-forward or feed-back signaling?}

Once ascertained that NOTCH induces DLL4 expression we wondered whether DLL4 overexpression could serve as a positive or a negative regulation mechanism of NOTCH signaling. NOTCH ligands can be shed from the plasma membrane and serve as inhibitor of NOTCH signaling. Thus, the potential negative effect of soluble DLL4 (sDLL4) on NOTCH signaling was tested in ECs (unpublished data Caolo et al 2010). Theoretically, sDLL4 would inhibit the DLL4-induced expression in cells cultured on immobilized DLL4 by competing with immobilized DLL4 for the same membrane bound NOTCH receptor without activating signaling. We found that the inhibition displayed by SDLL4 was not as effective as that observed with $\gamma$-secretases blockers. Furthermore, we were unable to detect any SDLL4 in immuno-precipitated medium from cells that were previously treated with immobilized DLL4 or NICD, which presented with higher DLL4 expression. These data, together with those from chapter 2, are in favor of a mechanism by which induced DLL4 is produced as an activating membrane bound ligand rather than a soluble inhibitor.

Stimulation of ECs with immobilized recombinant DLL4 triggered a positive feedforward loop that resulted in the activation of NOTCH signaling and subsequent upregulation of DLL4. Such mechanism was VEGF-signaling independent as the inhibition of VEGFRs did not prevent this effect (Chapter 2). The ability of an activated NOTCH receptor to induce the expression of its ligand DLL4 provides ECS with a mechanism by which NOTCH signaling might be distributed between communicating ECs. NOTCH signaling activation, initially potentiated by external stimuli such as VEGF, would induce DLL4 up-regulation and in turn NOTCH signaling and DLL4 expression in the surrounding cells ${ }^{23}$ (Figure 1). As DLL4-induced NOTCH signaling is essential for arterial specification, propagation of NOTCH signaling through the positive feed-forward loop, could be a suitable mechanism to induce and maintain arterial marker expression along a blood vessel. Besides inducing 
arterial marker expression DLL4-induced NOTCH signaling is required for regulating sprouting of newly forming vessels. Loss of DLL4-Notch signaling in vitro ${ }^{24}$ and in zebrafish and mouse models of angiogenesis led to ectopic sprouting and increased tip cell number ${ }^{7}, 11,25-27$. A mechanism, by which DLL4 expression and NOTCH signaling are propagated, would prevent filopodia extension in a subpopulation of cells rather than exclusively in the stalk cells immediately adjacent to the tip cells. A balance between tip cells and stalk cells will eventually contribute to a proper branching patterning and to the formation of a functional blood vessel network.

\section{VEGF induced NOTCH signaling and DLL4 overexpression by activating ADAM-10}

VEGF-induced RBP-Jk transactivation and NOTCH target gene HES-1 expression indicated that NOTCH signaling was activated directly downstream of VEGF. This activation was not secondary to DLL4 upregulation, suggesting that VEGF might stimulate NOTCH signaling in a ligand independent way (Chapter 2). Swendeman et al recently showed that VEGF-A enhanced shedding of its own receptor VEGFR-2 and co-receptor NP1 by activating ADAM-17 and ADAM-10, respectively ${ }^{28}$. As ADAMs are essential metalloproteinases involved in the activation of NOTCH signaling - mediating the first proteolytic cleavage of the receptor - we speculated that VEGF-A induced NOTCH signaling by activating ADAM. Accordingly, VEGF increased both the expression of ADAM-10 at mRNA and protein level as well as ADAM related protease activity in endothelial cells (Chapter 3 ). Furthermore, we showed that the link between VEGFR-2 signaling and ADAM activity is likely a direct result of VEGFR-2 phosphorylation and related kinase activity. In particular, the phosphorylation of tyrosine 1175 within the C-terminus recruits PLC $\gamma$, which mediates the activation of the mitogen-activated protein kinase (MAPK)/extracellular-signaling-regulated kinase-1/2 (Erk1/2) cascade and the phosphorylated Tyr1175 adaptor molecule Shb $^{29}$ that activates Phosphatidylinositol 3' kinase (PI3K). Both ERK1/2 and PI3K are essential kinases involved in cell growth, proliferation, differentiation, motility, survival and intracellular trafficking. Another important phosphorylation site in VEGFR-2 is Tyr1214 which initiates the p38-MAPK pathway $^{30}$. Blockage of these main signal transduction pathways showed that inhibition of the PI3K or ERK1/2 pathway prevented VEGF-A165 induced ADAM-10 activity, whereas p38 inhibition was without consequences. These results indicate that VEGF/VEGFR-2 signaling, through PI3K and ERK1/2 kinase pathways, specifically induced ADAM expression and activity, which subsequently mediates VEGFR-2 shedding, thus limiting the duration of signaling. As ADAMs mediate NOTCH signaling activation even in the absence of typical NOTCH ligands ${ }^{31}$, VEGFA induced ADAM activity will likely result in enhanced $\mathrm{NOTCH}$ signaling, with subsequent increase of DLL4 expression, and shutdown of VEGF signaling by VEGFR-2 shedding. In support of this model, we showed that VEGF induced NOTCH signaling and DLL4 expression was prevented by ADAM blockers addition (Chapter 2). 
This interrelation between VEGF and ADAM activity could explain the spatial and/or temporal regulation that causes the "salt and pepper" pattern of DLL4 expression and associated tip and stalk cell distribution described for the mouse retina model 11,32 . The VEGFRs expression appears to determine whether a cell will become a tip or a stalk cell. Computational models have been used to allow various combinations of ECs heterozygous for VEGFR-1 or VEGFR-2 to compete with wild-type cells for the tip position ${ }^{12,33,34}$. Cells heterozygous for Vegfr-2 showed poor contribution to the tip cell population and predominantly became stalk cells in competition with wildtype cells, whereas cells heterozygous for Vegfr-1 dominated the tip cell population. The balance of VEGFR-2 and VEGFR-1 expression in individual endothelial cells appears to affect their potential to become tips cell during sprouting angiogenesis. In response to VEGF, cells which are supposed to have high VEGFR-2 signal will upregulate DLL4 and adopt tip cells to initiate sprouting ${ }^{12}$. Thus it can be speculated that in these cells, VEGF/VEGFR-2 induced ADAM activates NOTCH signaling and secondarily DLL4 expression, initially independent of a NOTCH ligand as was proven by Delwig et $\mathrm{al}^{31}$. This assumption is in agreement with the observation that the surrounding stalk cells will -initially- present with low DLL4 levels. Subsequently, expressing DLL4 cells would induce NOTCH signaling and DLL4 expression in the neighbor cells. Eventually, activated NOTCH would turn down VEGFR-2 signaling ${ }^{8}$ and thus reduces sprouting.

Altogether, these data provide new insights into the molecular mechanism that characterizes VEGF and NOTCH signaling pathway interaction, indicating that VEGFA activates ADAMs, that will initiate NOTCH signaling. Eventually, cleaved NICD induces DLL4 upregulation initiating a feed-forward loop that results in the propagation of NOTCH signaling and ligand expression between communicating endothelial cells (Figure1). 


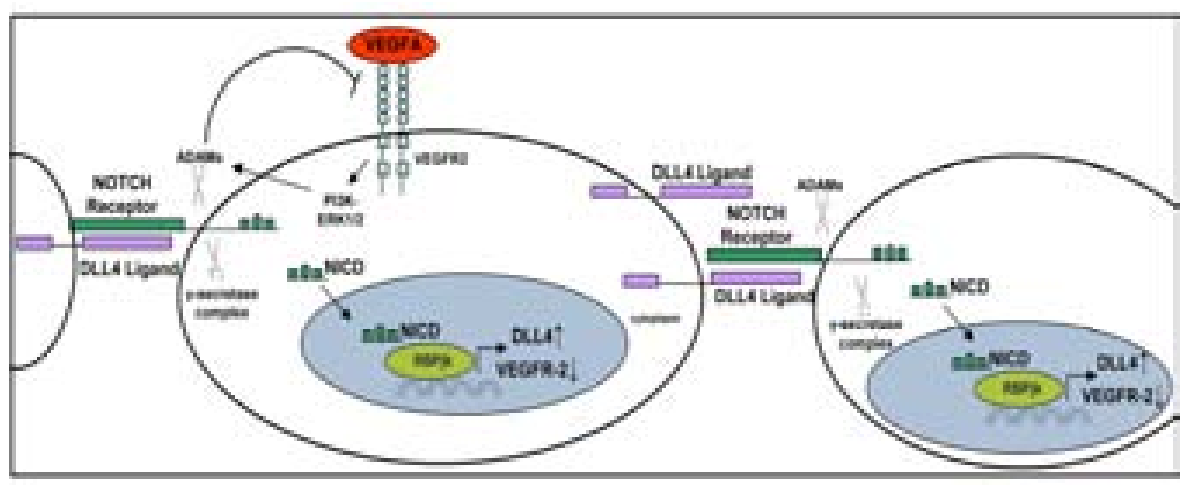

Figure 1 Proposed model of propagated NOTCH-signaling and DLL4 expression downstream of VEGF in Endothelial Cells. The stimulation of VEGFR-2 by VEGFA induces ADAM activity. Such process is mediated by PI3K and ERK1/2 intracellular kinase pathways. Activated ADAM will attenuate VEGFsignaling by shedding VEGFR-2, and will initiate NOTCH signaling, by shedding the extracellular region of the NOTCH receptor. Following ADAM, the $\gamma$-secretase complex mediates the second proteolytic cleavage of the NOTCH receptor leading to NICD release. Activated NICD translocates to the nucleus where it induces DLL4 and represses VEGFR-2 expression. Subsequently, newly expressed DLL4 activates the NOTCH receptor on the neighbor cell, which in turn up-regulates DLL4 expression. 


\section{Consequence of altered NOTCH signaling at the Tissue/Organ Level}

DLL4 blockade: potential therapeutic implication for inhibition of tumour growth Over the last years, research efforts have focused on the identification of new therapeutic agents that disrupt functional angiogenesis in tumours. The crucial role of DLL4 in regulating vascular development and its high expression level in tumour vasculature $^{9,35-39}$ make it a suitable therapeutic target. Indraccolo et al ${ }^{40}$ reported that the escape of human T-cell acute lymphoblastic leukaemia (T-ALL) cells or colorectal cancer cells from dormancy is associated with DLL4 expression in the tumour microenvironment and increased Notch3 signaling in tumour cells. Conversely, neutralization of DLL4 greatly reduced EC-induced Notch3 activation in T-ALL and blocked tumour growth ${ }^{40}$. Moreover, DLL4 seems to play a role in the differentiation of Bone Marrow (BM)-derive perycites/vSMCs during vasculogenesis in Ewing's Sarcoma ${ }^{41,42}$. The BM cells migrate into the tumour and differentiate into endothelial cells and perycites supporting the growth of the Ewing's sarcoma. Inhibition of DLL4 correlated with decreased numbers of BM-derived cells in tumour vessels and increased tumor hypoxia ${ }^{41,42}$. From these studies it emerges that DLL4 plays a role in tumour vasculature formation, suggesting that interference with DLL4/NOTCH signaling might provide an approach to prevent formation of functional vessels in tissue surrounding tumours ${ }^{43,44}$. Consistently, several recent studies revealed that inhibition of DLL4/NOTCH signaling reduced the growth of various solid tumours in mice ${ }^{45-52}$. As observed in zebrafish and in the retina mouse model, despite the increase in tumour blood vessels density following anti-DLL4 therapy, these vessels were not functional ${ }^{45-47}$.

Although most of the current therapies aim to block the VEGF pathway, not all tumours are responsive to VEGF blockers ${ }^{3}$. Remarkably, anti-DLL4 therapies were effective against tumours that were resistant to anti-VEGF treatment ${ }^{3}$. Differently, DLL4 inhibition increase vessels density by inducing branching ${ }^{45,48}$. Despite the increase in vessel number, these vessels are disorganized and poorly perfused resulting in tumour size degradation (Figure 2). Therefore DLL4 inhibition could provide synergistic effects when combined with anti-VEGF therapy against certain

tumours ${ }^{45,46,53}$, providing a more exciting approach to inhibit tumour growth ${ }^{54}$. Anti-DLL4 antibodies have recently entered clinical trials. However, the toxic side effects of DLL4 are still poorly understood ${ }^{55}$. Chronic DLL4 blockade has recently been reported to abnormally activate endothelial cells, disrupt normal organ homeostasis and produce significant pathology in multiple organs, inducing vascular neoplasms ${ }^{56}$. From this study, it emerges that a diligent strategy is required to use the DLL4-NOTCH signaling pathway as a powerful tool against tumours, avoiding undesired side effects. Moreover, our data provide evidence for a role of VEGFinduced ADAM activity in the NOTCH signaling activation. We showed that VEGFinduced DLL4 expression was prevented by ADAM blockers (Chapter 2). Interfering at the level of NOTCH receptor activation - i.e. by using ADAM inhibitors - might 
provide a more potent tool to prevent blood vessels formation in tumours, than sole DLL4 inhibition.

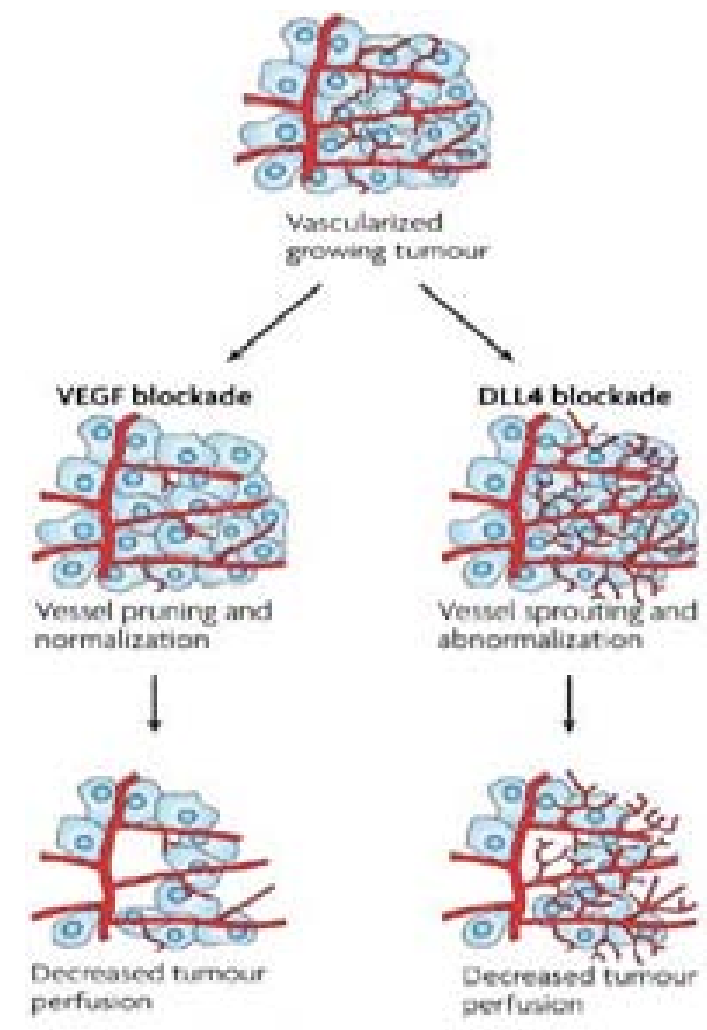

Reduced tumour growth
Figure 2 Comparison of VEGF versus DLL4-NOTCH inhibition in tumor angiogenesis. The tumor vasculature consists of actively growing and abnormal blood vessels. Addition of VEGF inhibitors results in decreased tumor perfusion and decreased tumor growth, although the tumor vasculature is normalized and the perfusion of vessels remains efficient. Conversely, DLL4 inhibitors addition results in increased tumour vessel density. However, these vessels are not functional, thus resulting in decreased tumour perfusion and growth (modified after Thurston Nature Review Cancer 2007). 
VEGF and NOTCH in coronary artery development: effects on endothelial differentiation and development of the media

Arterial specification, i.e. differentiation into venous or arterial endothelium is characterized by specific gene expression patterns. Arteries tend to express EphrinB2 and NP1 whereas venous expression includes EphB4 and COUP-TFII. VEGFinduced expression of DLL4 and increased NOTCH signaling results in overexpression of EphrinB2 and repression of EphB4 and COUP-TFII in endothelial cell and thus contributes to an arterial phenotype ${ }^{6,8,10,25,57}$. Ablation of COUP-TFII in endothelial cells likewise enables veins to acquire an arterial phenotype, including the expression of arterial markers NP1 and NOTCH signaling. Moreover, ectopic expression of COUP-TFII in ECs results in the fusion of veins and arteries in transgenic mouse embryos ${ }^{58}$.

In addition to the two VEGF receptors, several coreceptors (NP1, NP2 and heparin sulphate) are involved in the regulation of VEGF signaling in ECs ${ }^{59-62}$. NP1 and NP2 are membrane bound receptors containing a small cytoplasmatic domain lacking intrinsic catalytic functions ${ }^{63-66}$. NP1 is preferentially expressed in arterial endothelial cells ${ }^{67-69}$ and its mutation causes vascular defects in mouse embryos ${ }^{70}$. Although NP1 has initially been shown to guide the development of the nervous system, its importance in the development of the vascular system has been firmly established $^{63,65,66}$. Coexpression of neuropilins with VEGFR-2 in transfected cells enhances VEGF-A165 binding to VEGFR-2 ${ }^{68}$. Heparin sulphate is another crucial VEGFR-2 coreceptor ${ }^{59-61,}{ }^{71}$. Heparan sulfate proteoglycans (HSPGs) are transmembrane proteins and modulate the activity of a large number of secreted signaling molecules by stabilizing the complex between ligand and receptor ${ }^{72}$. HPSG can physically interact with VEGF modulating its interaction with VEGFR-2, and determining the duration of downstream signaling ${ }^{71}$. The VEGF ligands and isoforms display different interactions with the VEGF receptors and coreceptors, which results in diverse downstream effects. The three main human VEGF-A splice variants VEGF-A121, VEGF-A165 and VEGF-A189 are represented by Vegf120, Vegf164 and Vegf188, respectively in the mouse. We analyzed the effect of altered VEGFsignaling on NOTCH signaling and endothelial differentiation and on artery development by using a Vegf120/120 knock-in mouse model that lacks the other splice variants (Chapter 4). The coronary arterial differentiation was clearly altered in this mouse model. Arterial endothelial cells of Vegf120/120 transgenics showed decreased expression of several NOTCH members such as Notch1, DIl4 and Jag1 accompanied by a reduction of the arterial marker EphrinB2 and concomitant increase of the venous markers Coup-TFIl and EphB4. Conversely, the coronary venous ECs showed the reverse effect with increased expression of arterial markers and decreased venous marker expression. In vivo, in the Vegf120/120 mouse, a severe decrease in coronary arterial size with an increase in venous size was observed. We concluded that the altered expression of these endothelial markers 
was due to lack of the Vegf164 isoform. Stimulation of cultured human cardiac endothelial cells with either VEGF-A121 or VEGF-A165 confirmed the different effects of Vegf120 and Vegf164 on arterial specification observed in vivo. VEGFA165-stimulated ECs showed higher arterial marker EphrinB2 and lower venous marker COUP-TFII expression compared to VEGF-A121 stimulated cells. In Vegf120/120 mouse embryos, where Vegf120 is the sole VEGF isoform produced ${ }^{73}$, the VEGFR-2 and NOTCH signaling is likely negatively affected in arterial ECs. Moreover, due to its limited heparin-binding capacity, Vegf120 is more soluble than Vegf164 and will cause an ectopically higher VEGF concentration around the venous vessels than normally present. We assume that this increase in Vegf120 concentration will increase VEGF signaling as well and, subsequently will result in NOTCH signaling. Future research is required to unravel the link between EC signaling and the expression of $\mathrm{NOTCH}$ receptors in which VEGF will likely participate as a co-activator. From our study in Vegf120/120 mice it emerges that alteration of VEGF and NOTCH signaling during development affects maturation, morphology and function of the coronary system. Consistently, polymorphisms in the VEGF and VEGFR-2 genes in humans are associated with Kawasaki Disease ${ }^{74}$, in which children can develop coronary aneurysms upon inflammation ${ }^{75}$, and with coronary heart disease ${ }^{76}$. Our findings indicate an instructive role for VEGF isoforms distribution in coronary development which could be used for optimizing current revascularization therapies by targeting VEGF-signaling in the heart ${ }^{77}$.

Besides inducing arterial ECs specification, NOTCH signaling has been implicated in vSMC-development $^{78,79}$. Diminished arteriogenesis was also obvious in Vegf120/120 embryos, whereas coronary veins showed a gain of pericytes/vSMCs. The media of coronary arteries showed decreased number of cells expressing aSMA, NP1, DII1, Jagged2 and Notch3, whereas coronary veins showed an increase in cells positive for these markers (Chapter 4). On the basis of this data and of the reported link between NOTCH-signaling and SMC differentiation ${ }^{80}$, we proposed that alterations in the NOTCH-signaling pathway of ECs in Vegf120/120 mice, caused by defective VEGF-signaling, contributed to decreased differentiation of $\mathrm{VSMC}^{81}$ and therefore to aberrant arteriogenesis in these animals. A comparable link between SMCs differentiation induced by neighboring ECs through NOTCH signaling has been shown by Liu et al. They have shown that Jagged1 expressing endothelial cells can induce the expression of NOTCH-3 and Jagged1 in adjacent SMCs and this was essential for SMC differentiation ${ }^{80}$.

Altogether data from literature and our results underlie the crucial role of VEGFsignaling in inducing $\mathrm{NOTCH}$ expression and signaling, contributing to arterial differentiation of the endothelium. Conversely, the expression of COUP-TFII appears to be sufficient to repress VEGF and $\mathrm{NOTCH}$ signaling promoting a venous phenotype (Fi-gure 3). 

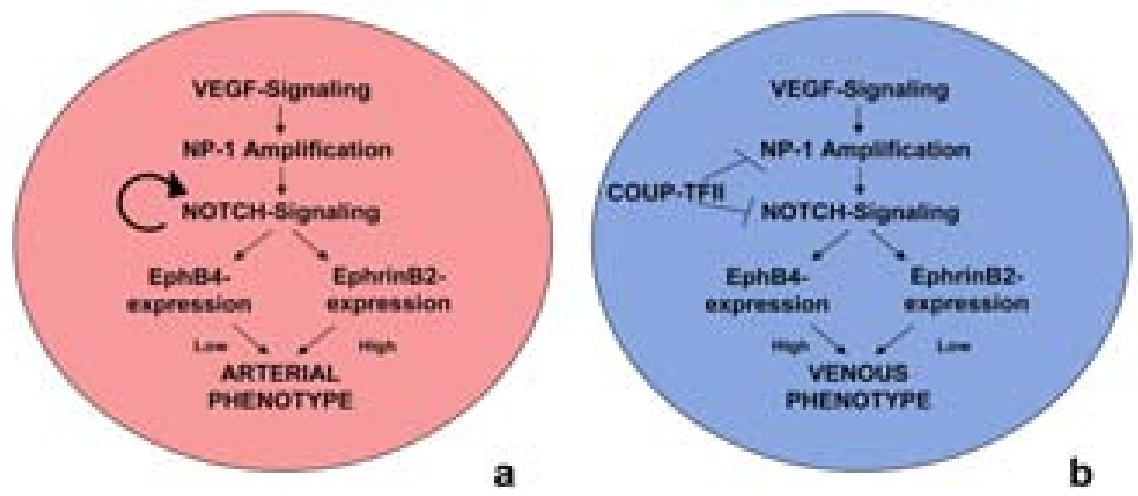

Adapted from You et al, Nature 2905

Figure 3 Role of VEGF-signaling in arterio-venous differentiation (a) High levels of VEGF-signaling, amplified by VEGF co-receptor NP1, leads to high NOTCH-signaling, which subsequently, induces the expression of the arterial EC-specific marker EphrinB2 and represses the expression of the vein-specific marker EphB4, resulting in an arterial phenotype of the EC. Notch activation also initiates a feedforward loop that results in the upregulation of its own signaling independent of VEGF-signaling. (b) The presence of COUP-TFII, a vein specific transcription factor, decreases NP-1 expression and negatively affects $\mathrm{NOTCH}$ expression and thus signaling. This gives rise to lower EphrinB2 and higher EphB4 levels, concomitant with a venous phenotype.

Inhibition of NOTCH-signaling by soluble Jagged1: a new tool to inhibit neointima formation

The behaviour of SMC in a blood vessel is strongly affected by their neighboring ECs. After vascular injury, SMCs in response to signals delivered from the injured endothelial and accumulating inflammatory cells start to migrate from the media to the intima and proliferate, forming an intima lesion in a process referred to as neointima formation. Lindner et al showed that the NOTCH circuit is activated following vascular injury ${ }^{82}$, and others reported a positive role for NOTCH in the neointima formation process ${ }^{83-85}$. Specifically, Li et al $^{83}$ demonstrated that Notch1 mediates SMC proliferation and neointima formation after vascular injury through a $\mathrm{NOTCH}$ specific CHF1/Hey2 signal pathway. The relation between $\mathrm{NOTCH}$ and neointima formation was further supported by analyses of Notch1+/- and SMCspecific Notch1 heterozygous deficient $\left(\mathrm{smN1}^{+/-}\right)$mice that showed decreased neointima formation after carotid artery ligation. Also Sakata et al ${ }^{84}$ reported that neointima formation was dramatically reduced in the Hey2-/- mice and that 
CHF1/Hey2-deficient VSMC showed decreased proliferation and ability to migrate in response to growth factors such as PDGF and heparin-binding epidermal growth factor (HB-EGF), which have been reported to accumulate at the site of vascular injury $^{86-88}$. Lamellopodia formation and membrane ruffling induced by these growth factors were reduced in Hey2 mutant VSMC compared to wild type SMC ${ }^{84}$. Moreover, Sos1 expression and Rac1 activation, essential proteins for cell proliferation and cell motility ${ }^{89,90}$, were reduced in Hey2 knockout VSMCs ${ }^{84}$. Other possible mechanisms that include regulation of cell cycle genes and apoptosis have been reported to play a role in vascular remodelling after injury. Wang et al (72) showed that the overexpression of another NOTCH target gene HRT1 (also named Hey1/HERP2/Hesr-1/CHF2) induces VSMC growth by inhibiting the expression of a crucial cell cycle inhibitor, p21 ${ }^{\text {WAF1/CIP1 }}$, and by up-regulating Akt expression/activity, a key modulator of apoptosis. Taken together, these data suggest that that inhibition of NOTCH signaling can prevent neointima formation.

Soluble forms of NOTCH ligands, Delta and Jagged, have been isolated and can serve as natural inhibitors of this pathway ${ }^{91,92}$. Lindner et al ${ }^{82}$ have shown that soluble Jagged1 (sJag1) ligand negatively affected migration of NIH3T3 cells by interfering with cell-matrix interaction and focal adhesion formation. A similar mechanism could affect SMC as well. We showed that sJag1 reduced neointima formation after vascular damage in a rat carotid artery injury model (Chapter 5 ). In this process sJag prevents the activation of NOTCH signaling by competing with the membrane bound ligand, thereby inhibiting NOTCH-signaling, SMC proliferation and migration as schematized in figure 4. We observed that among the NOTCH target genes, Herp2 was up-regulated in the media and neointima of injured vessel and was reduced in injured vessels that were treated with sJag1. The reduced neointima formation and Herp2 expression in animals treated with sJag strongly suggested a link between these two processes. Consistently, we showed that sJag was able to inhibit SMC migration and proliferation by interfering with Herp2 mediated NOTCH signaling. Decreased migration of the sJag treated SMC could be rescued by Herp2 overexpression, suggesting that Notch-Herp2 signaling is sufficient for SMC migration. Although further studies are needed to fully elucidate the molecular mechanism underlying the inhibition of NOTCH signaling by sJag1, it might provide a potential therapeutic tool to reduce restenosis in patients who have been subjected to percutaneous coronary intervention (PCI). The application of drug-eluting stent (DES) technology to improve clinical outcomes following $\mathrm{PCl}$ procedures represents one of the greatest successes in cardiology ${ }^{93,94}$. The use of stents capable to release agents such as antibodies and soluble ligands that interfere with NOTCH signaling, combined with antiflammatory drugs delivery, would probably benefit to limit restenosis after stent implantation. However, as previously discussed for DLL4, potentially toxicity of NOTCH ligand inhibition should be careful assessed. 


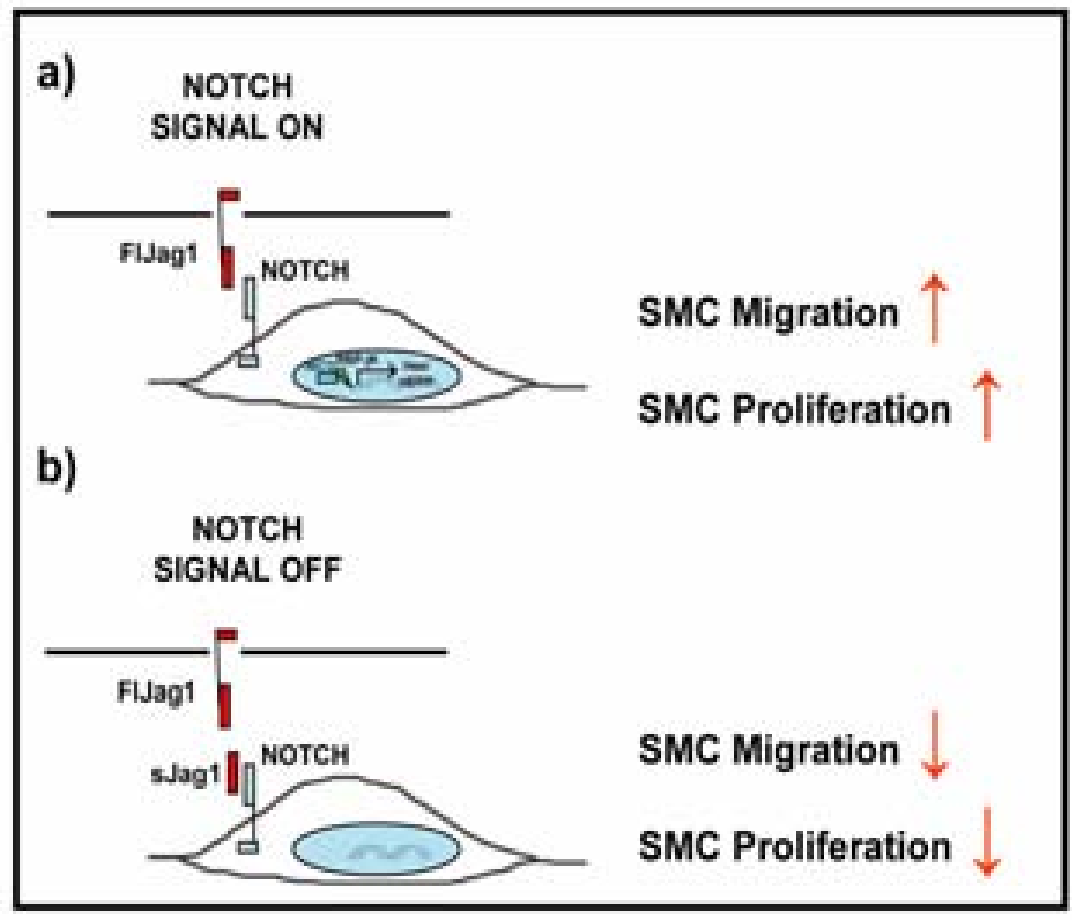

Figure 4 Model of NOTCH-signaling inhibition by sJag1 ligand. a) NOTCH receptor expressed by SMC interacts with Full length membrane bound Jag1 (FIJag1) located on the plasmatic membrane of the neighbor cell inducing NOTCH signaling. Next, NICD translocates to the nucleus where it interacts with its co-transcription factor RBP-Jk to initiate the transcription of HES/HERP target genes. Such activation positively affects SMC migration and proliferation. b) sJag1 competes with flJag1, subsequently preventing NOTCH signaling activation and SMC migration and proliferation.

\section{Conclusion}

Our results provide new insights in the molecular mechanisms that characterize the interaction between VEGF and NOTCH signaling in endothelial cells. We found that VEGF-induced DLL4 expression is NOTCH mediated. Furthermore, DLL4 initiated a feed-forward mechanism downstream of $\mathrm{NOTCH}$, which resulted in the upregulation of its own expression. These data indicate that VEGF-induced NOTCH signaling is not subsequent to DLL4 ligand overexpression per se (Chapter 2). Consistently, VEGF could induce the expression and the activity of ADAM-10, a crucial metalloproteinase involved in $\mathrm{NOTCH}$ receptor processing and activation (Chapter 3), providing an alternative mechanism by which VEGF can potentiate $\mathrm{NOTCH}$ receptor activation prior to and possibly independent of ligand overexpression. The interaction between VEGF and NOTCH signaling with respect to 
arterial specification has also been investigated (Chapter 4). We found that altered VEGF-signaling within the developing heart of the Vegf120/120 mouse embryo affected NOTCH signaling, endothelial differentiation and arteriogenesis, and as a consequence coronary artery development. Finally, we reported that sJag1 inhibited neo-intima formation in a rat carotid artery injury model (Chapter 5) following vascular injury by interfering with NOTCH-HERP2 signaling and, based on the in vitro data, we showed that this effect related to reduced SMC proliferation and migration. These results might provide a potential approach to reduce restenosis after $\mathrm{PCl}$. 


\section{References}

1 Gerhardt, H., VEGF and endothelial guidance in angiogenic sprouting, Organogenesis, 2008, 4: 241246.

2 Jakobsson, L., Bentley, K. and Gerhardt, H., VEGFRs and Notch: a dynamic collaboration in vascular patterning, Biochem Soc Trans, 2009, 37: 1233-1236.

3 Thurston, G. and Kitajewski, J., VEGF and Delta-Notch: interacting signalling pathways in tumour angiogenesis, Br J Cancer, 2008, 99: 1204-1209.

4 Trindade, A., Kumar, S. R., Scehnet, J. S., Lopes-da-Costa, L., Becker, J., Jiang, W., Liu, R., Gill, P. S. and Duarte, A., Overexpression of delta-like 4 induces arterialization and attenuates vessel formation in developing mouse embryos, Blood, 2008, 112: 1720-1729.

5 Suchting, S., Freitas, C., le Noble, F., Benedito, R., Breant, C., Duarte, A. and Eichmann, A., Negative regulators of vessel patterning, Novartis Found Symp, 2007, 283: 77-80; discussion 80-76, 238-241.

6 Harrington, L. S., Sainson, R. C., Williams, C. K., Taylor, J. M., Shi, W., Li, J. L. and Harris, A. L., Regulation of multiple angiogenic pathways by Dll4 and Notch in human umbilical vein endothelial cells, Microvasc Res, 2008, 75: 144-154.

7 Leslie, J. D., Ariza-McNaughton, L., Bermange, A. L., McAdow, R., Johnson, S. L. and Lewis, J., Endothelial signalling by the Notch ligand Delta-like 4 restricts angiogenesis, Development, 2007, 134: 839-844.

8 Williams, C. K., Li, J. L., Murga, M., Harris, A. L. and Tosato, G., Up-regulation of the Notch ligand Delta-like 4 inhibits VEGF-induced endothelial cell function, Blood, 2006, 107: 931-939.

9 Gale, N. W., Dominguez, M. G., Noguera, I., Pan, L., Hughes, V., Valenzuela, D. M., Murphy, A. J., Adams, N. C., Lin, H. C., Holash, J., Thurston, G. and Yancopoulos, G. D., Haploinsufficiency of deltalike 4 ligand results in embryonic lethality due to major defects in arterial and vascular development, Proc Natl Acad Sci U S A, 2004, 101: 15949-15954.

10 Liu, Z. J., Shirakawa, T., Li, Y., Soma, A., Oka, M., Dotto, G. P., Fairman, R. M., Velazquez, O. C. and Herlyn, M., Regulation of Notch1 and Dll4 by vascular endothelial growth factor in arterial endothelial cells: implications for modulating arteriogenesis and angiogenesis, Mol Cell Biol, 2003, 23: $14-25$.

11 Hellstrom, M., Phng, L. K., Hofmann, J. J., Wallgard, E., Coultas, L., Lindblom, P., Alva, J., Nilsson, A. K., Karlsson, L., Gaiano, N., Yoon, K., Rossant, J., Iruela-Arispe, M. L., Kalen, M., Gerhardt, H. and Betsholtz, C., Dll4 signalling through Notch1 regulates formation of tip cells during angiogenesis, Nature, 2007, 445: 776-780.

12 Bentley, K., Gerhardt, H. and Bates, P. A., Agent-based simulation of notch-mediated tip cell selection in angiogenic sprout initialisation, J Theor Biol, 2008, 250: 25-36.

13 Hellstrom, M., Phng, L. K. and Gerhardt, H., VEGF and Notch signaling: the yin and yang of angiogenic sprouting, Cell Adh Migr, 2007, 1: 133-136.

14 Suchting, S., Freitas, C., le Noble, F., Benedito, R., Breant, C., Duarte, A. and Eichmann, A., The Notch ligand Delta-like 4 negatively regulates endothelial tip cell formation and vessel branching, Proc Natl Acad Sci U S A, 2007, 104: 3225-3230.

15 Ferrara, N., Gerber, H. P. and LeCouter, J., The biology of VEGF and its receptors, Nat Med, 2003, 9: 669-676.

16 Park, J. E., Chen, H. H., Winer, J., Houck, K. A. and Ferrara, N., Placenta growth factor. Potentiation of vascular endothelial growth factor bioactivity, in vitro and in vivo, and high affinity binding to Flt-1 but not to Flk-1/KDR, J Biol Chem, 1994, 269: 25646-25654.

17 Waltenberger, J., Claesson-Welsh, L., Siegbahn, A., Shibuya, M. and Heldin, C. H., Different signal transduction properties of KDR and Flt1, two receptors for vascular endothelial growth factor, $J$ Biol Chem, 1994, 269: 26988-26995.

18 Hiratsuka, S., Minowa, O., Kuno, J., Noda, T. and Shibuya, M., Flt-1 lacking the tyrosine kinase domain is sufficient for normal development and angiogenesis in mice, Proc Natl Acad Sci U S A, 1998, 95: 9349-9354. 
19 Shalaby, F., Rossant, J., Yamaguchi, T. P., Gertsenstein, M., Wu, X. F., Breitman, M. L. and Schuh, A. C., Failure of blood-island formation and vasculogenesis in Flk-1-deficient mice, Nature, 1995, 376: $62-66$.

20 Seo, S., Fujita, H., Nakano, A., Kang, M., Duarte, A. and Kume, T., The forkhead transcription factors, Foxc1 and Foxc2, are required for arterial specification and lymphatic sprouting during vascular development, Dev Biol, 2006, 294: 458-470.

21 Diez, H., Fischer, A., Winkler, A., Hu, C. J., Hatzopoulos, A. K., Breier, G. and Gessler, M., Hypoxiamediated activation of Dll4-Notch-Hey2 signaling in endothelial progenitor cells and adoption of arterial cell fate, Exp Cell Res, 2007, 313: 1-9.

22 Hayashi, $\mathrm{H}$. and Kume, T., Foxc transcription factors directly regulate Dll4 and Hey2 expression by interacting with the VEGF-Notch signaling pathways in endothelial cells, PLoS One, 2008, 3: e2401.

23 Phng, L. K. and Gerhardt, H., Angiogenesis: a team effort coordinated by notch, Dev Cell, 2009, 16: 196-208.

24 Sainson, R. C., Aoto, J., Nakatsu, M. N., Holderfield, M., Conn, E., Koller, E. and Hughes, C. C., Cellautonomous notch signaling regulates endothelial cell branching and proliferation during vascular tubulogenesis, Faseb J, 2005, 19: 1027-1029.

25 Lobov, I. B., Renard, R. A., Papadopoulos, N., Gale, N. W., Thurston, G., Yancopoulos, G. D. and Wiegand, S. J., Delta-like ligand 4 (DII4) is induced by VEGF as a negative regulator of angiogenic sprouting, Proc Natl Acad Sci U S A, 2007, 104: 3219-3224.

26 Benedito, R., Trindade, A., Hirashima, M., Henrique, D., da Costa, L. L., Rossant, J., Gill, P. S. and Duarte, A., Loss of Notch signalling induced by Dll4 causes arterial calibre reduction by increasing endothelial cell response to angiogenic stimuli, BMC Dev Biol, 2008, 8: 117.

27 Siekmann, A. F. and Lawson, N. D., Notch signalling limits angiogenic cell behaviour in developing zebrafish arteries, Nature, 2007, 445: 781-784.

28 Swendeman, S., Mendelson, K., Weskamp, G., Horiuchi, K., Deutsch, U., Scherle, P., Hooper, A., Rafii, S. and Blobel, C. P., VEGF-A stimulates ADAM-17-dependent shedding of VEGFR2 and crosstalk between VEGFR2 and ERK signaling, Circ Res, 2008, 103: 916-918.

29 Holmqvist, K., Cross, M. J., Rolny, C., Hagerkvist, R., Rahimi, N., Matsumoto, T., Claesson-Welsh, L. and Welsh, M., The adaptor protein shb binds to tyrosine 1175 in vascular endothelial growth factor (VEGF) receptor-2 and regulates VEGF-dependent cellular migration, J Biol Chem, 2004, 279: 2226722275.

30 Lamalice, L., Houle, F., Jourdan, G. and Huot, J., Phosphorylation of tyrosine 1214 on VEGFR2 is required for VEGF-induced activation of Cdc42 upstream of SAPK2/p38, Oncogene, 2004, 23: 434445.

31 Delwig, A. and Rand, M. D., Kuz and TACE can activate Notch independent of ligand, Cell Mol Life Sci, 2008, 65: 2232-2243.

32 Hofmann, J. J. and Luisa Iruela-Arispe, M., Notch expression patterns in the retina: An eye on receptor-ligand distribution during angiogenesis, Gene Expr Patterns, 2007, 7: 461-470.

33 Bentley, K., Mariggi, G., Gerhardt, H. and Bates, P. A., Tipping the balance: robustness of tip cell selection, migration and fusion in angiogenesis, PLoS Comput Biol, 2009, 5: e1000549.

34 Jakobsson, L., Franco, C. A., Bentley, K., Collins, R. T., Ponsioen, B., Aspalter, I. M., Rosewell, I., Busse, M., Thurston, G., Medvinsky, A., Schulte-Merker, S. and Gerhardt, H., Endothelial cells dynamically compete for the tip cell position during angiogenic sprouting, Nat Cell Biol, 12: 943-953.

35 Hainaud, P., Contreres, J. O., Villemain, A., Liu, L. X., Plouet, J., Tobelem, G. and Dupuy, E., The role of the vascular endothelial growth factor-Delta-like 4 ligand/Notch4-ephrin B2 cascade in tumor vessel remodeling and endothelial cell functions, Cancer Res, 2006, 66: 8501-8510.

36 Mailhos, C., Modlich, U., Lewis, J., Harris, A., Bicknell, R. and Ish-Horowicz, D., Delta4, an endothelial specific notch ligand expressed at sites of physiological and tumor angiogenesis, Differentiation, 2001, 69: 135-144. 
37 Patel, N. S., Li, J. L., Generali, D., Poulsom, R., Cranston, D. W. and Harris, A. L., Up-regulation of delta-like 4 ligand in human tumor vasculature and the role of basal expression in endothelial cell function, Cancer Res, 2005, 65: 8690-8697.

38 Martinez, J. C., Muller, M. M., Turley, H., Steers, G., Choteau, L., Li, J. L., Sainson, R., Harris, A. L., Pezzella, F. and Gatter, K. C., Nuclear and membrane expression of the angiogenesis regulator deltalike ligand 4 (DLL4) in normal and malignant human tissues, Histopathology, 2009, 54: 598-606.

39 Jubb, A. M., Turley, H., Moeller, H. C., Steers, G., Han, C., Li, J. L., Leek, R., Tan, E. Y., Singh, B., Mortensen, N. J., Noguera-Troise, I., Pezzella, F., Gatter, K. C., Thurston, G., Fox, S. B. and Harris, A. L., Expression of delta-like ligand 4 (DII4) and markers of hypoxia in colon cancer, Br J Cancer, 2009, 101: 1749-1757.

40 Indraccolo, S., Minuzzo, S., Masiero, M., Pusceddu, I., Persano, L., Moserle, L., Reboldi, A., Favaro, E., Mecarozzi, M., Di Mario, G., Screpanti, I., Ponzoni, M., Doglioni, C. and Amadori, A., Cross-talk between tumor and endothelial cells involving the Notch3-Dll4 interaction marks escape from tumor dormancy, Cancer Res, 2009, 69: 1314-1323.

41 Schadler, K. L., Zweidler-McKay, P. A., Guan, H. and Kleinerman, E. S., Delta-like ligand 4 plays a critical role in pericyte/vascular smooth muscle cell formation during vasculogenesis and tumor vessel expansion in Ewing's sarcoma, Clin Cancer Res, 16: 848-856.

42 Stewart, K. S., Zhou, Z., Zweidler-McKay, P. and Kleinerman, E. S., Delta like ligand 4 - Notch signaling regulates $B M$-derived pericyte/vascular smooth muscle cell formation, Blood.

43 Yan, M. and Plowman, G. D., Delta-like 4/Notch signaling and its therapeutic implications, Clin Cancer Res, 2007, 13: 7243-7246.

44 Sainson, R. C. and Harris, A. L., Anti-Dll4 therapy: can we block tumour growth by increasing angiogenesis?, Trends Mol Med, 2007, 13: 389-395.

45 Noguera-Troise, I., Daly, C., Papadopoulos, N. J., Coetzee, S., Boland, P., Gale, N. W., Lin, H. C., Yancopoulos, G. D. and Thurston, G., Blockade of Dll4 inhibits tumour growth by promoting nonproductive angiogenesis, Nature, 2006, 444: 1032-1037.

46 Ridgway, J., Zhang, G., Wu, Y., Stawicki, S., Liang, W. C., Chanthery, Y., Kowalski, J., Watts, R. J., Callahan, C., Kasman, I., Singh, M., Chien, M., Tan, C., Hongo, J. A., de Sauvage, F., Plowman, G. and Yan, M., Inhibition of Dll4 signalling inhibits tumour growth by deregulating angiogenesis, Nature, 2006, 444: 1083-1087.

47 Scehnet, J. S., Jiang, W., Kumar, S. R., Krasnoperov, V., Trindade, A., Benedito, R., Djokovic, D., Borges, C., Ley, E. J., Duarte, A. and Gill, P. S., Inhibition of Dll4-mediated signaling induces proliferation of immature vessels and results in poor tissue perfusion, Blood, 2007, 109: 4753-4760.

48 Thurston, G., Noguera-Troise, I. and Yancopoulos, G. D., The Delta paradox: DLL4 blockade leads to more tumour vessels but less tumour growth, Nat Rev Cancer, 2007, 7: 327-331.

49 Segarra, M., Williams, C. K., Sierra Mde, L., Bernardo, M., McCormick, P. J., Maric, D., Regino, C., Choyke, P. and Tosato, G., DII4 activation of Notch signaling reduces tumor vascularity and inhibits tumor growth, Blood, 2008, 112: 1904-1911.

50 Yamanda, S., Ebihara, S., Asada, M., Okazaki, T., Niu, K., Ebihara, T., Koyanagi, A., Yamaguchi, N., Yagita, H. and Arai, H., Role of ephrinB2 in nonproductive angiogenesis induced by Delta-like 4 blockade, Blood, 2009, 113: 3631-3639.

51 Oishi, H., Sunamura, M., Egawa, S., Motoi, F., Unno, M., Furukawa, T., Habib, N. A. and Yagita, H., Blockade of delta-like ligand 4 signaling inhibits both growth and angiogenesis of pancreatic cancer, Pancreas, 39: 897-903.

52 Hoey, T., Yen, W. C., Axelrod, F., Basi, J., Donigian, L., Dylla, S., Fitch-Bruhns, M., Lazetic, S., Park, I. K., Sato, A., Satyal, S., Wang, X., Clarke, M. F., Lewicki, J. and Gurney, A., DLL4 blockade inhibits tumor growth and reduces tumor-initiating cell frequency, Cell Stem Cell, 2009, 5: 168-177.

53 Li, J. L., Sainson, R. C., Shi, W., Leek, R., Harrington, L. S., Preusser, M., Biswas, S., Turley, H., Heikamp, E., Hainfellner, J. A. and Harris, A. L., Delta-like 4 Notch ligand regulates tumor angiogenesis, improves tumor vascular function, and promotes tumor growth in vivo, Cancer Res, 2007, 67: 11244-11253. 
$54 \mathrm{Li}, \mathrm{J}$. L. and Harris, A. L., Crosstalk of VEGF and Notch pathways in tumour angiogenesis: therapeutic implications, Front Biosci, 2009, 14: 3094-3110.

55 Li, J. L., Jubb, A. M. and Harris, A. L., Targeting DLL4 in tumors shows preclinical activity but potentially significant toxicity, Future Oncol, 6: 1099-1103.

56 Yan, M., Callahan, C. A., Beyer, J. C., Allamneni, K. P., Zhang, G., Ridgway, J. B., Niessen, K. and Plowman, G. D., Chronic DLL4 blockade induces vascular neoplasms, Nature, 463: E6-7.

57 Zhang, G., Zhou, J., Fan, Q., Zheng, Z., Zhang, F., Liu, X. and Hu, S., Arterial-venous endothelial cell fate is related to vascular endothelial growth factor and Notch status during human bone mesenchymal stem cell differentiation, FEBS Lett, 2008, 582: 2957-2964.

58 You, L. R., Lin, F. J., Lee, C. T., DeMayo, F. J., Tsai, M. J. and Tsai, S. Y., Suppression of Notch signalling by the COUP-TFII transcription factor regulates vein identity, Nature, 2005, 435: 98-104.

59 Neufeld, G., Cohen, T., Gengrinovitch, S. and Poltorak, Z., Vascular endothelial growth factor (VEGF) and its receptors, Faseb J, 1999, 13: 9-22.

60 Matsumoto, T. and Claesson-Welsh, L., VEGF receptor signal transduction, Sci STKE, 2001, 2001: re21.

61 Grunewald, F. S., Prota, A. E., Giese, A. and Ballmer-Hofer, K., Structure-function analysis of VEGF receptor activation and the role of coreceptors in angiogenic signaling, Biochim Biophys Acta, 1804: 567-580.

62 Stuttfeld, E. and Ballmer-Hofer, K., Structure and function of VEGF receptors, IUBMB Life, 2009, 61: 915-922.

63 Staton, C. A., Kumar, I., Reed, M. W. and Brown, N. J., Neuropilins in physiological and pathological angiogenesis, J Pathol, 2007, 212: 237-248.

64 Fujisawa, H., Kitsukawa, T., Kawakami, A., Takagi, S., Shimizu, M. and Hirata, T., Roles of a neuronal cell-surface molecule, neuropilin, in nerve fiber fasciculation and guidance, Cell Tissue Res, 1997, 290: 465-470.

65 Zachary, I., Neuroprotective role of vascular endothelial growth factor: signalling mechanisms, biological function, and therapeutic potential, Neurosignals, 2005, 14: 207-221.

66 Neufeld, G., Cohen, T., Shraga, N., Lange, T., Kessler, O. and Herzog, Y., The neuropilins: multifunctional semaphorin and VEGF receptors that modulate axon guidance and angiogenesis, Trends Cardiovasc Med, 2002, 12: 13-19.

67 Moyon, D., Pardanaud, L., Yuan, L., Breant, C. and Eichmann, A., Plasticity of endothelial cells during arterial-venous differentiation in the avian embryo, Development, 2001, 128: 3359-3370.

68 Soker, S., Takashima, S., Miao, H. Q., Neufeld, G. and Klagsbrun, M., Neuropilin-1 is expressed by endothelial and tumor cells as an isoform-specific receptor for vascular endothelial growth factor, Cell, 1998, 92: 735-745.

69 Pan, Q., Chathery, Y., Wu, Y., Rathore, N., Tong, R. K., Peale, F., Bagri, A., Tessier-Lavigne, M., Koch, A. W. and Watts, R. J., Neuropilin-1 binds to VEGF121 and regulates endothelial cell migration and sprouting, J Biol Chem, 2007, 282: 24049-24056.

70 Kawasaki, T., Kitsukawa, T., Bekku, Y., Matsuda, Y., Sanbo, M., Yagi, T. and Fujisawa, H., A requirement for neuropilin-1 in embryonic vessel formation, Development, 1999, 126: 4895-4902.

71 Jakobsson, L., Kreuger, J., Holmborn, K., Lundin, L., Eriksson, I., Kjellen, L. and Claesson-Welsh, L., Heparan sulfate in trans potentiates VEGFR-mediated angiogenesis, Dev Cell, 2006, 10: 625-634.

72 Esko, J. D. and Selleck, S. B., Order out of chaos: assembly of ligand binding sites in heparan sulfate, Annu Rev Biochem, 2002, 71: 435-471.

73 Houck, K. A., Leung, D. W., Rowland, A. M., Winer, J. and Ferrara, N., Dual regulation of vascular endothelial growth factor bioavailability by genetic and proteolytic mechanisms, J Biol Chem, 1992, 267: 26031-26037.

74 Kariyazono, H., Ohno, T., Khajoee, V., Ihara, K., Kusuhara, K., Kinukawa, N., Mizuno, Y. and Hara, T., Association of vascular endothelial growth factor (VEGF) and VEGF receptor gene polymorphisms with coronary artery lesions of Kawasaki disease, Pediatr Res, 2004, 56: 953-959. 
75 Freeman, A. F. and Shulman, S. T., Recent developments in Kawasaki disease, Curr Opin Infect Dis, 2001, 14: 357-361.

76 Wang, Y., Zheng, Y., Zhang, W., Yu, H., Lou, K., Zhang, Y., Qin, Q., Zhao, B., Yang, Y. and Hui, R., Polymorphisms of KDR gene are associated with coronary heart disease, J Am Coll Cardiol, 2007, 50: 760-767.

77 Yla-Herttuala, S., Rissanen, T. T., Vajanto, I. and Hartikainen, J., Vascular endothelial growth factors: biology and current status of clinical applications in cardiovascular medicine, J Am Coll Cardiol, 2007, 49: 1015-1026.

78 Limbourg, A., Ploom, M., Elligsen, D., Sorensen, I., Ziegelhoeffer, T., Gossler, A., Drexler, H. and Limbourg, F. P., Notch ligand Delta-like 1 is essential for postnatal arteriogenesis, Circ Res, 2007, 100: 363-371.

79 High, F. A., Zhang, M., Proweller, A., Tu, L., Parmacek, M. S., Pear, W. S. and Epstein, J. A., An essential role for Notch in neural crest during cardiovascular development and smooth muscle differentiation, J Clin Invest, 2007, 117: 353-363.

80 Liu, H., Kennard, S. and Lilly, B., NOTCH3 expression is induced in mural cells through an autoregulatory loop that requires endothelial-expressed JAGGED1, Circ Res, 2009, 104: 466-475.

81 Liu, W., Parikh, A. A., Stoeltzing, O., Fan, F., McCarty, M. F., Wey, J., Hicklin, D. J. and Ellis, L. M., Upregulation of neuropilin-1 by basic fibroblast growth factor enhances vascular smooth muscle cell migration in response to VEGF, Cytokine, 2005, 32: 206-212.

82 Lindner, V., Booth, C., Prudovsky, I., Small, D., Maciag, T. and Liaw, L., Members of the Jagged/Notch gene families are expressed in injured arteries and regulate cell phenotype via alterations in cell matrix and cell-cell interaction, Am J Pathol, 2001, 159: 875-883.

83 Li, Y., Takeshita, K., Liu, P. Y., Satoh, M., Oyama, N., Mukai, Y., Chin, M. T., Krebs, L., Kotlikoff, M. I., Radtke, F., Gridley, T. and Liao, J. K., Smooth muscle Notch1 mediates neointimal formation after vascular injury, Circulation, 2009, 119: 2686-2692.

84 Sakata, Y., Xiang, F., Chen, Z., Kiriyama, Y., Kamei, C. N., Simon, D. I. and Chin, M. T., Transcription factor $\mathrm{CHF} 1 /$ Hey2 regulates neointimal formation in vivo and vascular smooth muscle proliferation and migration in vitro, Arterioscler Thromb Vasc Biol, 2004, 24: 2069-2074.

85 Wang, W., Prince, C. Z., Hu, X. and Pollman, M. J., HRT1 modulates vascular smooth muscle cell proliferation and apoptosis, Biochem Biophys Res Commun, 2003, 308: 596-601.

86 Heldin, C. H. and Westermark, B., Mechanism of action and in vivo role of platelet-derived growth factor, Physiol Rev, 1999, 79: 1283-1316.

87 Miyagawa, J., Higashiyama, S., Kawata, S., Inui, Y., Tamura, S., Yamamoto, K., Nishida, M., Nakamura, T., Yamashita, S., Matsuzawa, Y. and et al., Localization of heparin-binding EGF-like growth factor in the smooth muscle cells and macrophages of human atherosclerotic plaques, J Clin Invest, 1995, 95: 404-411.

88 Igura, T., Kawata, S., Miyagawa, J., Inui, Y., Tamura, S., Fukuda, K., Isozaki, K., Yamamori, K., Taniguchi, N., Higashiyama, S. and Matsuzawa, Y., Expression of heparin-binding epidermal growth factor-like growth factor in neointimal cells induced by balloon injury in rat carotid arteries, Arterioscler Thromb Vasc Biol, 1996, 16: 1524-1531.

89 Moore, K. A., Sethi, R., Doanes, A. M., Johnson, T. M., Pracyk, J. B., Kirby, M., Irani, K., GoldschmidtClermont, P. J. and Finkel, T., Rac1 is required for cell proliferation and G2/M progression, Biochem J, 1997, 326 ( Pt 1): 17-20.

90 Anand-Apte, B., Zetter, B. R., Viswanathan, A., Qiu, R. G., Chen, J., Ruggieri, R. and Symons, M., Platelet-derived growth factor and fibronectin-stimulated migration are differentially regulated by the Rac and extracellular signal-regulated kinase pathways, J Biol Chem, 1997, 272: 30688-30692.

91 Qi, H., Rand, M. D., Wu, X., Sestan, N., Wang, W., Rakic, P., Xu, T. and Artavanis-Tsakonas, S., Processing of the notch ligand delta by the metalloprotease Kuzbanian, Science, 1999, 283: 91-94. 
92 Duarte, M., Kolev, V., Kacer, D., Mouta-Bellum, C., Soldi, R., Graziani, I., Kirov, A., Friesel, R., Liaw, L., Small, D., Verdi, J., Maciag, T. and Prudovsky, I., Novel cross-talk between three cardiovascular regulators: thrombin cleavage fragment of Jagged1 induces fibroblast growth factor 1 expression and release, $\mathrm{Mol}$ Biol Cell, 2008, 19: 4863-4874.

93 Htay, T. and Liu, M. W., Drug-eluting stent: a review and update, Vasc Health Risk Manag, 2005, 1: 263-276.

94 Montone, R. A., Ferrante, G., Baca, M. and Niccoli, G., Predictive value of C-reactive protein after drug-eluting stent implantation, Future Cardiol, 6: 167-179. 


\section{Summary}

In this thesis, the role of VEGF and NOTCH Signaling Pathways in cardiovascular development and function has been investigated. The effects of VEGF on DLL4 expression, NOTCH signaling and ADAM activity in endothelial cells (ECs) have been explored. Also, the consequences of altered NOTCH signaling for vessel function have been studied at cellular as well as the organ level.

In Chapter 1, a general introduction of this thesis is provided. The interaction between VEGF and NOTCH signaling pathway is described together with their effects on arterial endothelial cell differentiation and sprouting. The NOTCH signaling role in SMCs behavior is introduced along with potential implications of NOTCH signaling modulation for vascular remodeling.

In Chapter $\mathbf{2}$ we provide new insights into the molecular interaction between the NOTCH and VEGF signaling pathways in ECs. We demonstrated that VEGF-induced DLL4 expression depends on NOTCH activation in HCMvECs. Accordingly, NOTCH signaling was activated by VEGF, as was shown by overexpression of the NOTCH target gene HES-1 and transactivation of a promoter containing multiple RBP-Jk binding sites. Next, activation of $\mathrm{NOTCH}$ signaling, by NICD overexpression or recombinant DLL4 stimulation, induced the expression of DLL4 ligand. Also, we reported that NICD-induced DLL4 promoter activity was dependent on RBP-Jk sites but independent of the FOXC2 binding site, previously reported to be required in regulating DLL4 expression downstream VEGF. Moreover, constitutively active FOXC2 did not affect DLL4 expression.

We speculated that the induction of DLL4 by activated NOTCH might provide a positive feed-forward signaling mechanism that propagates $\mathrm{NOTCH}$-signaling between neighboring ECs. Indeed, DLL4-eGFP transfected ECs induced DLL4 expression in non-transfected cells in close vicinity in a NOTCH-dependent way. As DLL4 regulates crucial processes in vascular growth, such as EC sprouting and arterial specification, a deeper understanding of the molecular mechanisms underling its regulation will undoubtedly help to development new strategies for the treatment of blood vessel-related pathologies.

In Chapter 3 the VEGF effect on ADAM-10 activation, an essential metalloproteinases involved in NOTCH processing and activation, has been studied. Using a Yeast-two-hybrid assay, ADAM-10 was identified as a novel binding partner of VEGFR-2. Such interaction was further confirmed by immuno-precipitation experiments. Remarkably, VEGF stimulation induced ADAM-10 expression and its activity in ECs. VEGF-activated ADAM-10 mediated ectodomain shedding of VEGFR2, providing a negative feed-back mechanism that shuts down VEGF signaling in ECs. VEGF induced-ADAM-10 also resulted in cleavage of VE-cadherin, increasing 
vascular permeability and, facilitated EC migration. Therefore, VEGF increased vascular permeability in an ADAM -dependent manner and inhibition of ADAM-10 reduced EC migration and chemotaxis. For the first time, we reported that ADAM-10 is expressed in human atherosclerotic lesions and associated with plaque progression and neovascularization. Altogether, these data provide evidence of a role for ADAM10 in atherosclerosis and neovascularization. As ADAM-10 is involved in $\mathrm{NOTCH}$ signaling proteolysis and activation, and VEGF has been reported to activate NOTCH signaling, these results provide an important link between VEGF and NOTCH signaling activation as well.

In Chapter 4 the role of VEGF and $\mathrm{NOTCH}$ signaling in arterial endothelial differentiation is addressed. In mutant Vegf120/120 mouse embryos, the endothelial cells of coronary arteries failed to gain a proper arterial phenotype (i.e. high level of activated Notch1, Jagged1, DII4 and ephrinB2 and low levels of VEGFR2, EphB4 and COUP-TFII), while the endothelial cells of coronary veins showed ectopic expression of the arterial markers together with low expression of the venous markers. Moreover, the media of coronary arteries was underdeveloped with decreased number of pericytes expressing aSMA, NP1, DII1, Jagged2 and Notch3, whereas coronary veins showed an increase in cells positive for these markers.

Vegf is mainly expressed in the area where coronary arteries develop in normal mouse embryos. This part of the coronary system is expected to be subjected to high levels of VEGF-signaling due to retention of the larger VEGF splicing variants in the heparin-containing extracellular matrix and to NP-1-modulated amplification. In the subepicardially located coronary veins therefore. low VEGF-signaling levels are expected. Due to lack of retention of VEGF120 in the extracellular matrix and lack of NP-1-mediated amplification in Vegf120/120 mouse embryo, the inverse is likely the case, leading to the observed coronary anomalies. These data imply that VEGF and the downstream NOTCH signaling are essential for arterial endothelial differentiation and arteriogenesis with consequences for coronary artery development and performance.

In Chapter 5 the negative effect of a soluble NOTCH ligand, sJag1, on NOTCH signaling and neointima formation is studied. Rat carotid arteries subjected to balloon injury showed increase of Notch1, Notch3 and Jagged-1 expression at day 3 and 14 post-injury. In these injured vessels, Notch signaling was activated as was shown by increased expression of the NOTCH target-gene Herp2. We found that adenoviral-sJag1 transfection reduced neo-intima formation in the carotid artery and enhanced re-endothelialization, whereas adenoviral-full length Jagged-1 (FIJag1) or -LacZ had no effect. As neointima is the result of uncontrolled proliferation and migration of SMCs, we analyzed the effect of sJag1 on SMCs behavior by transducing CASMCs with sJag1. In line with our expectations, sJag1 reduced both migration and proliferation of CASMCs compared to FI-Jag1 or LacZ used as control, 
whereas no effect on ECs was observed. Moreover, injury-induced Herp2 expression was dramatically reduced in injured vessels treated with adenoviral-sJag1. Accordingly, CASMCs infected with Ad-sJag1 or treated with sJag recombinant protein showed decreased Herp2 expression compared to their respective controls. These observations prompted us to speculate that sJag1 prevents SMC migration and proliferation by interfering with Herp2 mediated NOTCH signaling. In line with these speculations, overexpression of Herp2 rescued the reduced migratory capacity of sJag1-treated CASMCs. Thus, sJag1 can inhibit neo-intima formation after balloon injury by decreasing SMC proliferation and migration through interference with NOTCH-Herp2 signaling.

Chapter 6 provides a general discussion of the data presented in this thesis. First we discussed the new insights in the NOTCH and VEGF signaling molecular interaction provided by our studies. In particular, the major role of VEGFR-2 and the existence of a positive feed-forward loop - that involves activated NOTCH signaling - in VEGFinduced DLL4 expression was examined. The activation of ADAM-10 by VEGF and its implication in NOTCH signaling activation downstream of VEGF has been discussed. Second we focused on the consequence of altered NOTCH signaling at the tissue/organ level. The DLL4 blockade as potential therapeutic tool in tumor growth was introduced. In addition, the implication of VEGF and NOTCH signaling pathway in arterial differentiation and development of coronary artery was discussed. Finally we considered therapeutic use of sJag to inhibit neointima formation following PCl. 


\section{Samenvatting}

In dit proefschrift zijn de vascular endothelial growth factor (VEGF) en NOTCH signaal wegen bestudeerd wegens hun rol in de ontwikkeling en functie van het bloedvat stelsel. Meer specifiek hebben we het effect van VEGF op de expressie van Delta-like 4 (DLL4) en de activiteit van NOTCH en "a desintegrin associated metalloprotease" (ADAM) in endotheel cellen onderzocht. Tenslotte hebben we enkele functionele consequenties van veranderde NOTCH activatie bekeken in vasculaire cellen als ook in bloedvaten.

Hoofdstuk 1 leidt het onderwerp van het promotieonderzoek in. Hierin worden de VEGF en NOTCH signaalwegen beschreven en wordt tevens het verband geschetst met differentiatie en het spruiten - bijvoorbeeld bij nieuwvorming van bloedvatenvan slagaderlijk endotheel. Vervolgens wordt de rol van NOTCH activatie op het gedrag van gladde spiercellen besproken en de mogelijk gevolgen daarvan voor vaatwand remodellering.

In Hoofdstuk 2 verschaffen we nieuw inzicht in de moleculaire interactie tussen de $\mathrm{NOTCH}$ and VEGF signaalwegen in endotheelcellen. In humane coronaire microvasculaire endotheel cellen (HCMvECs) hebben we laten zien dat $\mathrm{NOTCH}$ activatie nodig was voor de expressie van DLL4 door VEGF. Deze NOTCH activatie bleek uit de inductie van het NOTCH afhankelijke "hairy enhancer of split" (HES-1) door VEGF, evenals uit reporter assays met een promotor rijk aan RBP-Jk transactivatie domeinen. Het intracellulaire domein van NOTCH (NICD) dat verantwoordelijk is voor de transcriptie-activatie, leidde ook tot overexpressie van DLL4, evenals recombinant DLL4 (rDLL4) zelf dat als ligand van NOTCH zorgt voor NOTCH activatie. In tegenstelling tot studies van anderen hebben we geen aanwijzingen gevonden dat FOXC2 betrokken is bij DLL4 expressie. Promotor constructen zonder het FOX bindende domein lieten toch activatie zien, terwijl overexpressie van FOXC2 niet leidde tot DLL4 expressie.

De observatie dat rDLL4 aanzet tot DLL4 expressie suggereert een positieve terugkoppelingsfunctie waarin initiëring van $\mathrm{NOTCH}$ activatie snel leidt tot het overdragen van de activatie op naburige cellen. Het bleek inderdaad dat DLL4 overexprimerende cellen leidde tot NOTCH activatie van naburige cellen met DLL4 expresssie tot gevolg.

Omdat DLL4 betrokken is bij spruiten van nieuwe bloedvaten en bij differentiatie in slagaderlijke bloedvaten zal beter inzicht in dit proces kunnen leiden tot nieuwe therapieën.

In Hoofdstuk $\mathbf{3}$ is het effect van VEGF op activatie van ADAM10 bestudeerd. ADAM10 is een essentieel metalloproteinase voor NOTCH activatie. In een 'Yeast-twohybrid' systeem werd ADAM10 als bindingspartner van VEGFR-2 geïdentificeerd en 
dit werd bevestigd door immuunprecipitatie. VEGF stimuleerde ADAM-10 expressie en activiteit in endotheelcellen. Aangezien ADAM10 op zijn beurt leidt tot afbraak van het extracellulaire deel van VEGR-2 en daarmee de receptor inactiveert, lijkt ADAM-10 onderdeel te zijn van een negatief terugkoppelingsmechanisme. VEGF geïnduceerde ADAM-10 resulteerde ook in de afbraak van VE-cadherin en dus in verhoogde permeabiliteit en endotheelcel migratie. Deze laatste effecten bleken ook door ADAM-17 te kunnen worden bewerkstelligd. ADAM-10 blijkt in human atherosclerotische plaques voor te komen en is geassocieerd met plaque-groei en vaatnieuwvorming in de plaque. Aangezien ADAM-10 activatie noodzakelijk is voor NOTCH activatie en VEGF ook leidt tot activatie van NOTCH, is het waarschijnlijk dat ADAM-10 een noodzakelijk onderdeel is van de VEGF-NOTCH as.

In Hoofdstuk 4 wordt de rol van VEGF en NOTCH in slagaderlijke differentiatie gedurende de embryonale ontwikkeling besproken. In Vegf120/120 muize-embryo's vormen ondergaan endotheelcellen onvoldoende slagaderlijke differentiatie. Hun expressie van slagaderlijke kenmerken als Notch1, Jagged1, Dll4 en EphrinB2 bleef laag, terwijl aderlijke kenmerken als EphB4 en COUP-TFII hoog bleven. Aan de andere kant, werden plaatselijk slagaderlijke kenmerken gevonden in typische aderlijke posities als de kransaderen. De media van de slagaders ontwikkelde zich ook onvoldoende met verlaagde aantallen pericyten die aSMA, NP1, Dll1, Jagged2 en Notch3 exprimeren, terwijl de aderen juist meer van deze cellen recruteerden. In normale embryo's wordt vegf vooral gevonden in de gebieden waar kransslagaderen worden gevormd. Deze hoge expressie leidt tot retentie van heparine bindende varianten van vegf (zoals vegf164, 188) en deze hoge expressie wordt mogelijk nog versterkt door neuropilin-1 (NP-1) gemedieerde activatie. De aderen ontwikkelen zich in de subepicardiale laag waar lage vegf spiegels heersen. Echter, Vegf120 bindt niet aan de heparine en wordt ook niet versterkt door NP-1, zodat slagaderlijke differentiatie verminderd is. Deze observaties impliceren dat VEGF en NOTCH activatie essentieel zijn voor slagaderlijke differentiatie en daarmee voor de functie van het bloedvat stelsel als geheel.

Hoofdstuk 5 beschrijft de inhibitie van NOTCH door een niet membraan gebonden ligand, het zogenaamde 'soluble Jagged-1' (sJag1), die in een model van vaatwandschade leidt tot verminderde vorming van een neo-intima. Vaatwandschade leidt tot expressie van NOTCH1, NOTCH3 en Jagged-1 in 3-14 dagen na de schade. Bovendien was NOTCH geactiveerd aangezien we Herp-2 in deze vaten konden aantonen. Behandeling met sJag-1 verminderde de vorming van neointima en versnelde de regeneratie van endotheel in deze halsslagaderen terwijl membraan gebonden Jagged-1 of LacZ geen effect hadden. Neointima is het gevolg van gladde spiercel migratie, proliferatie en matrixeitwit synthese. In gladde spiercellen van kransslagaderen (CASMCs) leidde sJag1 inderdaad tot verminderde proliferatie en migratie alsook tot verminderde Herp-2 expressie. Gelijktijdige overexpressie van Herp-2 kon de sJag-1 verminderde migratie en proliferatie 
tegengaan, wat suggereert dat $\mathrm{NOTCH}$ activatie inderdaad door sJag1 werd geïnhibeerd. Er werd dus geconcludeerd dat sJag1 neointima vorming remt door de migratie en proliferatie van gladde spiercellen te verminderen doordat $\mathrm{NOTCH}-$ Herp-2 activatie wordt verhinderd.

Hoofdstuk 6 is een algemene bespreking van alle data die in dit proefschrift worden gepresenteerd. Eerst worden de nieuwe inzichten in de interactie tussen VEGF en NOTCH signaalwegen besproken. In het bijzonder wordt het positieve terugkoppelingsmechanisme van VEGF, NOTCH activatie en DLL4 expressie bediscussieerd. ADAM-10 activatie lijkt noodzakelijk te zijn voor dit proces, terwijl afbraak van de VEGF receptor zou kunnen leiden tot een natuurlijke rem op dit systeem. Vervolgens hebben we de rol van veranderde NOTCH activatie in cel- en orgaan-systemen besproken, waarbij DLL4 blokkade therapeutisch wordt ingezet bij kanker. Bovendien blijkt tijdens de embryonale ontwikkeling NOTCH activatie nodig te zijn voor normale ontwikkeling van met name het slagaderlijke deel van het vaatstelsel. Tenslotte hebben we de mogelijkheden besproken om remming van $\mathrm{NOTCH}$ activatie therapeutisch in te zetten tegen excessieve neointima vorming na $\mathrm{PCl}$. 


\section{List of Abbreviations}

\begin{tabular}{|c|c|}
\hline ADAM & A Disintegrin and Metalloprotease \\
\hline AEC & Arterial Endothelial Cell \\
\hline ANK & Ankyrin Repeats \\
\hline AoA & Aortic Arch \\
\hline AV & Arterial Venous \\
\hline bFGF & basic Fibroblast Growth Factor \\
\hline BM & Bone Marrow \\
\hline CADASIL & $\begin{array}{l}\text { Cerebral Autosomal Dominant Arteriopathy with Subcortical } \\
\text { Infarcts and Leukoencephalopathy }\end{array}$ \\
\hline CASMC & Coronary Artery Smooth Muscle Cell \\
\hline $\mathrm{CL}$ & Cardiac Lymphatic \\
\hline $\mathrm{Cl}$ Notch1 & Cleaved Notch1 \\
\hline $\mathrm{CM}$ & Compact Myocardium \\
\hline Co-R & Co-Repressor \\
\hline COUP-TFII & Chicken Ovalbumin Upstream Promoter Transcription Factor II \\
\hline CSL & CBF1/RBPJk, $\underline{\mathrm{S}} \mathrm{u}(\mathrm{H}), \underline{\operatorname{Lag} 1}$ \\
\hline CV & Coronary Vein \\
\hline DAB & 3,3'- Diaminobenzidine \\
\hline DAPT & $\begin{array}{l}\mathrm{N} \text {-[N-(3,5-Difluorophenacetyl)-L-alanyl]-S-phenylglycine-t-butyl } \\
\text { ester }\end{array}$ \\
\hline DIl or DLL & Delta-like \\
\hline DMEM & Dulbecco's Modified Eagle Medium \\
\hline DMSO & Dimethyl sulfoxide \\
\hline DSL & Delta/Serrate/Lag-2 \\
\hline$E$ & Embryonic Day \\
\hline ECs & Endothelial Cells \\
\hline EDTA & Ethylenediaminetetraacetic acid \\
\hline EGF & Epidermal Growth Factor \\
\hline eGFP & Enhanced Green Fluorescent Protein \\
\hline EGTA & Ethylene Glycol Tetraacetic Acid \\
\hline EPC & Endothelial Progenitors Cell \\
\hline EPDC & Epicardium-derived Cell \\
\hline Eph & Ephrin Receptor \\
\hline ERK/MAPK & $\begin{array}{l}\text { Extracellular signal-regulated kinase / mitogen-activated protein } \\
\text { kinase }\end{array}$ \\
\hline FBE & Forkhead Binding Element \\
\hline Fl-Jag 1 & Full Length Jagged1 \\
\hline
\end{tabular}


FLK1

FLT1

FOXC

GADPH

HB-EGF

HCAEC

HCMvEC

HEPES

HERP

HES

HIF

HR

HRT (HEY)

HSPG

HUVEC

II-6

IVS

Jag or JAG

KDR

LCV

LDL

LYVE

MAML1

MOPS

NCK

NECD

NICD

NLS

NO

NP

OPA

p21 WAF1/CIP1

PAEC

PBS

PC

$\mathrm{PCl}$

PCNA

PDGF

PDZ

PEST

PFA
Fetal Liver Kinase 1

FMS-like Tyrosine Kinase

Forkhead Box C

Glyceraldehyde 3-Phosphate Dehydrogenase

Heparin-Binding Epidermal Growth Factor

Human Coronary Arterial Endothelial Cell

Human Cardiac Microvascular Endothelial Cell

N'-2-Hydroxyethylpiperazine-N'-2 Ethanesulphonic Acid

Hairy Enhancer of Split-related Repressor Protein

Hairy/Enhancer of Split

Hypoxia Inducible Factor

Heat Retrieval

Hairy Related Transcription Factor

Heparine Sulphate Proteoglycans

Human Umbilical Vein Endothelial Cell

Interleukin 6

Intervetricular Septum

Jagged

Kinase insert Domain Receptor

Left Cardinal Vein

Low-Density Lipoprotein

Lymphatic Vessel Endothelial Hyaluronan Receptor

Mastermind like protein 1

3-(N-morpholino)propanesulfonic acid

Non-catalytic Region of Tyrosine Kinase Adaptor Protein

NOTCH Extracellular Domain

NOTCH Intracellular Domain

Nuclear Localization Sequence

Nitric Oxide

Neuropilin

Glutamine-rich domain

cyclin-dependent kinase inhibitor

Porcine Aortic Endothelial Cell

Phosphate-Buffered Saline

Pericardial Cavity

Percutaneous Coronary Intervention

Proliferating Cell Nuclear Antigen

Platelet-Derived Growth Factor

Postsynaptic density; Disc large; Zonula Occludens-1

Proline/ Glutaminc Acid/ Serine/ Threonine-rich

Paraformaldehyde 


\begin{tabular}{|c|c|}
\hline $\mathrm{PI} 3 \mathrm{~K}$ & Phosphatidylinositol 3' Kinase \\
\hline PIGF & Placenta Growth Factor \\
\hline PLC $\gamma$ & Phospholipase C gamma \\
\hline PMA & Phorbol 12-Myristate 13-Acetate \\
\hline P38-MAPK & p38/ Mitogen-Activated Protein Kinase \\
\hline PMSF & Phenylmethanesulfonylfluoride \\
\hline POUFUT1 & Protein O-Fucosyl Transferase \\
\hline PT & Pulmunary Trunk \\
\hline PVDF & Polyvinylidene Difluoride \\
\hline qPCR & quantitative Polymerase Chain Reaction \\
\hline RA & Right Atrium \\
\hline RAM & RBP-Jk Associated Module \\
\hline RCA & Right Coronary Artery \\
\hline rDLL4 & Recombinant Delta like 4 \\
\hline RBPJk & Recombining Binding Protein suppressor of hairless \\
\hline $\mathrm{RCV}$ & Right Cardinal Vein \\
\hline RIPA & Radioimmunoprecipitation Assay \\
\hline RNAi & RNA interference \\
\hline RT & Reverse Transcriptase \\
\hline RTK & Receptor Tyrosine Kinase \\
\hline RUNX2 & Runt related transcription factor 2 \\
\hline RV & Right Ventricle \\
\hline SDS-PAGE & Sodium Dodecyl Sulfate - Poly Acrylamide Gel Electroforese \\
\hline SiRNA & Small interfering RNA \\
\hline sJag1 & soluble Jagged1 \\
\hline $\operatorname{SM} 22 \alpha$ & Smooth Muscle 22 alpha \\
\hline SMA & Smooth Muscle Actin \\
\hline SM-MHC & Smooth Muscle Myosin Heavy Chain \\
\hline SRF & Serum Response Factor \\
\hline TACE & TNF- $\alpha$ Converting Enzyme \\
\hline TBS & Tris-Buffered Saline \\
\hline TBST & Tris-Buffered Saline Tween \\
\hline TGF $\beta$ & Transforming Growth factor $\beta$ \\
\hline TNF $\alpha$ & Tumor Necrosis Factor alpha \\
\hline TOF & Tetralogy of Fallot Growth Factor \\
\hline VCACs & Ventriculo-coronary Arterial Communication \\
\hline VEC & Veins Endothelial Cell \\
\hline VE-cadherin & Vascular Endothelial - cadherin \\
\hline VEGF & Vascular Endothelial Growth Factor \\
\hline VEGF & Vascular Endothelial Growth Factor Receptor \\
\hline vSMCs & vascular Smooth Muscle Cells \\
\hline
\end{tabular}




\section{List of Publications}

1. van den Akker N.M., Caolo V., Wisse L.J., Peters P.P., Poelmann R.E., Carmeliet P., Gittenberger-de Groot A.C., Molin D.G. Developmetal coronary maturation is disturbed by aberrant cardiac VEGF-expression and Notch-Signaling. Cardiovasc. Res. 2008;78:366-75.

2. Donners M.M., Wolfs I.M., Olieslagers S., Mohammadi-Motahhari Z., Tchaikovski V., Heeneman S., van Buul J.D., Caolo V., Molin D.G., Post M.J., Waltenberger J. ADAM-10 is a novel mediator of VEGF-induced endothelial cell function in angiogenesis and associated with atherosclerosis. Arterioscler. Thromb. Vasc. Biol. 2010;30:2188-95.

3. Caolo V., van den Akker N.M.S., Verbruggen S., Donners M.M.P.C., Swennen G., Schulten H., Waltenberger J., Post M.J., Molin D.G.M. Feed-forward signaling by membrane bound ligand receptor circuit: The case of NOTCH-DELTA LIKE 4 in endothelial cells. Journal of Biological Chemistry, 2010;285:40681-89.

4. Caolo V., Schulten H.M., Zhuang Z.W., Murakami M., Wagenaar A., Verbruggen S., Molin D.G.M., Post M.J. Soluble Jagged-1 inhibits Neointima Formation by attenuating Notch-Herp2 signaling. Arterioscler. Thromb. Vasc. Biol. 2011, In press.

\section{Published Abstracts}

Vincenza Caolo, Henny Schulten, Sanne Verbruggen, Geertje Swennen, Johannes Waltenberger, Daniël G. Molin, Mark J. Post. Abstract 3941: VEGF-A Induces Arterial Endothelial Differentiation By Increasing Both NOTCH-expression And NOTCHrelated Signaling. Circulation. 2008;118:S_506. 


\section{Acknowledgments}

Finally, it is the moment to thank all people, who, in their own way, contributed to the realization of this dissertation.

First of all, my deep gratitude goes to my supervisor, Mark Post. Mark, I am very grateful to you for the opportunity you gave me to work in your group, for your continuous inputs and feedback on my work and for the interesting and fruitful discussions that accompanied our weekly meetings. Thanks for believing in me when I barely could express myself in English, that was (and still is) our only option. It has been a privilege to work with you and it is an honor to continue this working experience with you for the coming three years.

My sincere gratitude goes to my co-supervisor, Daniël Molin. Daniël, as you know the interaction between us has never been smooth! However, without that large number of heated discussions we had, we would not be what we are today: a winning combination at work! Thanks for your constant support: whenever I had a doubt or a question, I could come or write to you and always get an answer. Thanks to your precious help, I could attend very prestigious conferences. I am sorry for that time the passport controllers never let me take the plane to LA and you had to present in my stead. I hope our future collaboration will be as productive as the previous one.

A "big Thanks" goes to all the people of my group. Sanne, thanks for all times I came to you at the last minute because I needed more aliquots of materials or an extra pair of hands to run my experiments. You were always very patient and willing to help me. Geertje, you have been a great colleague all over these years, by professionally and personally supporting me. Sorry, for all the times you had to help me to interpret the results of my odd WBs and thanks for all the times you have told me "come on Vincenza, you can do it!", when I was discouraged. Allard, thanks for all the support with the animal work and for making me sick with your movies during our lab meetings at 8:30 in the morning, showing every single detail of your surgical interventions in animals, I still remember all of them. I would also like to thank you, Irma. You are a great roommate and a very smart colleague. Your curiosity and questioning ability make you able to continuously instigate very interesting scientific (and not only) discussions! Nastaran, since the first day you arrived we got along very well. I still remember your smiling face when you introduced yourself. Creativity and humor are your best qualities, besides many others. Felix, I still have to work hard to convince you I am the "senior PhD" and when I speak you should "shut uppy". Thanks for creating that friendly atmosphere when you are in the lab. Mark, although you are the new entry of the group, it looks like you have been working with us since ever. Your intelligence and elegance make 
you one of my favorite colleagues. Thanks also to Nick and Patricia for the technical support they provided me during the first year of my PhD experience. Thanks to you, Henny, with whom I have worked for the first three years of my PhD. I will never forget your way of miming snails...that was impressive! Nynke, a "huge thanks" goes to you. Our collaboration started before I even personally knew you. It has always been a pleasure to work with you. You have an extraordinary capability to identify and quickly solve problems.

I am grateful to Prof. Johannes Waltenberger and Marjo Donners for the fruitful collaboration we had during these years. Thanks for sharing with us your expertise.

Another great colleague I need to thank is you, Judith. We have shared the office for more than three years, until the time came when you had to leave to USA for your Postdoctoral Fellow. I have been missing you so much. You helped me since the first day I arrived in Maastricht, offering me hospitality at you place.

Thanks to you, Ali. For every time I asked you to solve my computer issues. You always have kindly helped me. Of course, everything has a price to pay, but your requests were so easy to satisfy: sometimes you asked a tiramisu', other times a dinner at my place.

I would like also to extend "my thanks" to the Secretaries of my Department, Bianca and Vivian and our former secretary Sonia. Since the first day I arrived, I was very impressed by your kindness and helpfulness.

I am also grateful to all the people of my Department, who provided me with essential feedback during our "Physiology Meetings". A special "thanks" goes to Ger van de Vusse, with whom I had the pleasure to discuss my research progress in few occasions during these years. Thanks also for the great time that I spent with you and your family.

I also would like to thank the members of the assessment committee for reading this manuscript and their positive comments: Prof. Jo de Mey, Prof. Erik Biessen, Prof. Michael Simons and Dr. Guillaume van Eys.

A special "Thanks" to Guillaume (Gino) and Jannette. Our friendship started with our trip to Australia almost three years ago. We spent 18 beautiful days together traveling around, from Sydney to Ayers Rock to Cairns to Brisbane. I will never forget the amazing things we have seen together. Especially that time I had to face you, Gino, in your scary red pyjamas and I could not escape anywhere as we were in the middle of the desert! I will never forget your frightened faces after my rather rash driving performance! At that time, to mortify me not further, you did not express any comment. However, I do not remember my-self driving for the remaining time of our trip. Thanks for every time you invited me for dinner and I could taste Jannette's beautiful Indonesian food. For every Sinterklaas I had the honor to celebrate with you and your wonderful family.

Thanks also to you Peter Frederik. You have impressed me since the first moment I had the chance to speak with you. You are a great scientist, a great musician and a 
great artist, I also had the opportunity to experience you as a great friend. I will always remember that spring afternoon when you took me by motorbike to that peculiar restaurant in Tong Herve for dinner. Since then we had few more occasions to meet each other and it has always been a great pleasure to spend time with you. I also want to thank all the friends that I met in Maastricht and made my stay here an absolutely unforgettable and exciting experience. Anna, I had the pleasure to become your friend after few months I arrived in Maastricht. You have always been very supportive and your help has been of vital importance to deal with the many difficulties I was facing at the beginning of this first experience abroad. Thanks also for introducing me to the little, but vibrant Spanish community of Maastricht, where I met many other friends. Among them, I found you Alvaro. A consolidated and true friendship binds us since almost five years. You are an exceptionally smart person and I feel privileged to be your friend. Thanks for all the beautiful and funny days we have spent together and for every time I came to cry on your shoulder. Thanks to you, Benoit. Although you live in Rotterdam and we do not have many occasions to meet, the moments I spend with you are just hilarious! I also want to thank you guys: Nieves, Lorena, Jordi, Nicolas for the innumerable dinners and funny evenings we spent together. Thanks to you, Christina. For all the lunch's we had together at Uni speaking about our "crazy" PhD experience, also for the great times we have spent hanging out together. Thanks also to Craig (Stanny), Victoria and Toddy for being always so friendly to me. Un immenso grazie va a te, Mariana. Tu sei stata una persona importantissima in questa esperienza "Maastrichtiana". Nonostante sapessi che avresti dovuto seguire la tua strada, quando hai deciso di partire, ho provato un grande senso di vuoto. Ero consapevole che sarebbe stato difficile, se non impossibile, trovare un'altra amica premurosa e divertente come te! Mi manchi tanto, ma e' bello vedere, quando ci incontriamo, che un forte legame di amicizia ancora ci lega! Grazie a te, Jacopo, e alla tua inestimabile simpatia. Siamo subito andati d'accordo, sara' perche' siamo compaesani ("capisc' a me").

Dopo un breve periodo di sconforto, dovuto alla partenza della maggior parte degli amici piu' affezionati, ecco che il sole ricomincia a splendere! E' stata una salvezza incontrare Voi Amici Miei. Arianna, grazie per essere sempre cosi' solare e ottimista. Riesci sempre a farmi sorridere anche quando lo sconforto dilaga. Grazie per le mille giornate passate insieme a parlare di tutto e di niente. E sopratutto grazie per aiutarmi a prendere la vita con piu' leggerezza! Stefano, grazie per tutte le volte che Arianna mi ha invitato a cena e tu hai dovuto cucinare e anche se non mi fai assistere alle partite di calcio, io sempre cantero': "sognavo questa Roma e Roma $c^{\prime} \mathrm{e}^{\prime \prime \prime}$. Epo (Andrea), grazie per sopportare con pazienza i miei sfotto' e per farmi sentire cosi' ignorante quando inizi a parlare di musica e cinematografia. Francesco, grazie per trattarmi sempre con estrema spontaneita', spesso dimenticando che sono una ragazza. Ma soprattutto grazie per la tua simpatia e per farmi provare quello strano senso di ebbrezza ogni volta che vengo in macchina con te! Els, grazie 
per far fronte con estrema abilita' alla mia sarcastica ironia. Due righe vanno obbligatoriamente a te, Giacomo. Grazie per avere allietato, movimentato e qualche volta scombussolato le nostre serate! Grazie a te Elena. Tu sei un vulcano di simpatia e quando ci sei tu il divertimento e' garantito! Emanuela, nonostante ti conosca da poco e ti abbia incontrato ancora meno (visto che sei sempre in giro per il mondo!), sin dal primo istante mi sono trovata in perfetta sintonia con te. Mi auguro di poter trascorrere piu' tempo con te in futuro!

Un gigantesco grazie va te, Laila, amica di sempre. Grazie per esserci ogni volta che ho bisogno di te! Anche se migliaia di kilometri ci separano, sappiamo sempre quando l'una ha bisogno dell'altra semplicemente attraverso i nostri sogni. Il legame che ci lega e' straordinario!

Un enorme "Grazie" va alla mia eccezionale famiglia! Grazie per avermi supportato durante tutti questi anni. Mamma, non $c^{\prime} e^{\prime}$ stato giorno in cui non ci siamo telefonicamente sentite. Tu sei sempre li' ad ascoltarmi, a confortarmi e a darmi il giusto consiglio per affrontare ogni situazione. Papa', anche se non lo dimostri, so che sei estramente orgoglioso di me. Me ne rendo conto quando faccio ritorno a Scario e la gente del paese, superinformata su tutte le mie piu' recenti esperienze lavorative, mi ripete che non fai altro che parlare di me. Grazie va a mio fratello Giuseppe. Non perdi occassione per dirmi: "continua a fare quello che fai e ricorda che in Italia non ci devi tornare". Non puoi neanche lontanamente immaginare quanto queste parole mi abbiano aiutato a tenere duro nei momenti piu'difficili. Grazie anche a mio fratello Gianluca. E' incredibile constatare, che per quanto abbia lasciato Scario piu' di dieci anni fa, tu ancora cerchi ostinatamente di persuadermi a tornare, promettendomi l'appartementino ristrutturato e un lavoro alla "Clinica Verde". Grazie per venirmi a prendere all'aereoporto ogni volta che ritorno in Italia, con quella premura da fratello maggiore che ti contraddistingue, e per i tuoi generosi regali! Un grazie va anche a Zia Maria, Zio Sandro, Sandra. E di sicuro un grandissimo grazie a te, Cinzia. Grazie per tutte le volte che sono venuta a Milano, per tutte le volte che verro' in futuro e per il supporto che mi dai con le tue periodiche videochiamate su Skype.

My acknowledgments end with You, Craig. Since almost three years ago, when I met you, my life deeply improved. Your cares, your love and your delicious food are my daily source of energy. Since we met we have done so many things together that it is impossible to enlist and squeeze them in few lines of this acknowledgments. Every day I spend with you looks better than the previous one and I wish many other days will come in which I will stand by your side. And as Steve sings: "I Feel Like This Is The Beginning, Though I've Loved You For A Million Years"... 


\section{Curriculum Vitae}

Vincenza Caolo was born on March $13^{\text {th }} 1982$, in Sapri, Italy. After finishing the high school at the Liceo Scientifico "Galileo Galilei" in Sapri in 2000, she studied Molecular Biology at the Bologna University. In March 2006 she obtained her Master Degree cum laude. Since July 2006 she was appointed as a Marie Curie PhD student at the Physiology Department of the Cardiovascular Research Institute Maastricht (CARIM) of Maastricht University under the supervision of Prof. Mark J. Post. Since January 2011 she is working as Postdoctoral Research Fellow at the Physiology Department of Maastricht University. 